Fernanda Marcello

\title{
Past to Future South Atlantic Overturning Circulation: upper-ocean pathways and low-frequency variability
}

Thesis submitted to the Oceanographic Institute of the University of São Paulo in partial fulfillment of the requirements for the degree of Doctor of Philosophy in Oceanography, with emphasis in Physical Oceanography.

Advisor:

Prof.. Dr. a Ilana Wainer

Co-advisor:

Prof.. Dr. ${ }^{\text {a }}$ Regina R. Rodrigues

São Paulo 
One of the great challenges in this world

is knowing enough about a subject to think you're right,

but not enough about the subject to know you're wrong.

$\sim$ Neil deGrasse Tyson 


\section{Contents}

\begin{tabular}{lll}
\hline Acknowledgements & v
\end{tabular}

\begin{tabular}{lll}
\hline Abstract vi & vi
\end{tabular}

Resumo vii

List of Figures viii

List of Tables $\quad$ x

List of Abbreviations $\quad$ xi

1 Introduction 1

1.1 Preamble and Thesis Outline . . . . . . . . . . . . . . . . . . . . 1

1.2 Theoretical Background . . . . . . . . . . . . . . . . . . . . . . . 4

1.2.1 The Global Overturning Circulation and the Climate System 4

1.2.2 The Atlantic Meridional Overturning Circulation . . . . . . . 20

1.2.3 The South Atlantic as a critical crossroad for the AMOC . . 22

1.3 Motivation and Research Questions . . . . . . . . . . . . . . . . . 25

1.4 Objectives . . . . . . . . . . . . . . . . . . . . . . 27

$\begin{array}{lll}2 & \text { Datasets } & 28\end{array}$

3 South Atlantic Subtropical Gyre late twentieth century changes 31

3.1 Introductory remarks . . . . . . . . . . . . . . . . . . . . . . . . . . 31

3.2 Data and Methods . . . . . . . . . . . . . . . . . . . 33

3.2 .1 SBL definition . . . . . . . . . . . . . . . . . . . . . . . 34

3.2 .2 SASG dynamical indices $\ldots \ldots . . \ldots 34$

3.3 Results . . . . . . . . . . . . . . . . . . . . . . . . . . . . . 35

3.3.1 Long-term changes in SASG dynamics . . . . . . . . . . 35

3.3 .2 Long-term changes in the SBL . . . . . . . . . . . . . . . . 42

3.3.3 Long-term changes in SAO current transports. . . . . . . . 45

3.4 Conclusions and Discussion . . . . . . . . . . . . . . . . . . . . . . 49 
4 South Atlantic surface boundary current system during the LM 51

4.1 Introductory remarks . . . . . . . . . . . . . . . . . . . 51

4.2 Data and Methods . . . . . . . . . . . . . . . . . . 54

4.3 Results . . . . . . . . . . . . . . . . . . . . . . . . . . 55

4.3.1 Sea Surface Temperature field . . . . . . . . . . . . . . . . 55

4.3.2 Horizontal Velocity Field and Volume Transports . . . . . . 57

4.3 .3 Wind Stress Curl field . . . . . . . . . . . . . . . 66

4.4 Conclusions and Discussion . . . . . . . . . . . . . . . . . . . 67

5 Upper-limb pathways of the South Atlantic Overturning Circulation 70

5.1 Introductory remarks . . . . . . . . . . . . . . . . . . 70

5.2 Data and Methods . . . . . . . . . . . . . . 76

5.3 Results $\ldots \ldots \ldots \ldots \ldots \ldots \ldots \ldots \ldots \ldots \ldots$

5.3.1 Characterization of the General Mean Circulation Field . . . 78

5.3 .2 Overall Large-Scale Projected Changes . . . . . . . . . . . 83

5.3 .3 Drake Passage, ACC and southern SASG. . . . . . . . . . 85

5.3.4 Agulhas Leakage, SASG recirculation and AMOC . . . . . 96

5.4 Conclusions and Discussion . . . . . . . . . . . . . . . . . . . . 111

$\begin{array}{lll}6 & \text { Concluding Remarks } & 117\end{array}$

$\begin{array}{lll}7 & \text { Scientific Progress } & 122\end{array}$

\begin{tabular}{lll}
\hline 8 & Other publication results & 126
\end{tabular}

8.1 Warm Deep Water variability during the Last Millennium in the CESM-LME: pre-Industrial scenario vs. late 20th century changes 126

8.2 Long-term regional sea level changes from CMIP6 projections . . 128

8.3 21st century projected changes in the Weddell Sea Antarctic Slope Current in a Downscaling Experiment . . . . . . . . . . . . . . . . 130

9 List of Publications $\quad 131$

$\begin{array}{lr}\text { References } & 132\end{array}$

\begin{tabular}{lr}
\hline Appendix l & 169
\end{tabular} 


\section{Agradecimentos}

Em primeiro lugar, é claro, agradeço minha orientadora, a prof. Ilana Wainer, por sempre acreditar em mim, me motivar e me encorajar - além da paciência para eternas discussões e para me ensinar a fazer ciência com transparência e integridade.

Agradeço todos os membros do Laboratório $\mathrm{OC}^{2}$, com quem já tive ótimos momentos e os quais sempre estiveram disponíveis para me ouvir e para discutir ciência.

Agradeço minha co-orientadora, prof. Regina R. Rodrigues, por ter guiado minha trajetória acadêmica desde a graduação, me incentivando, apoiando minhas decisões e fornecendo todo o suporte necessário.

Ao Instituto Oceanográfico da USP pela oportunidade de realização do curso de pós-graduação e à todos os funcionários que trabalham para mantê-lo em ótimo funcionamento, mesmo durante a atual pandemia.

À Fundação de Amparo à Pesquisa do Estado de São Paulo (FAPESP), pelo financiamento desta pesquisa (processo $\mathrm{n}^{\circ}$ 2017/16511-5) e pela oportunidade de crescimento na ciência e na vida acadêmica.

O presente trabalho também foi realizado com o apoio e financiamento parcial do Conselho Nacional de Desenvolvimento Científico e Tecnológico (CNPq).

Expresso minha gratidão por toda minha família, principalmente meus pais, Roberto e Vânia, e Nathalia, minha irmã - responsáveis por eu ser quem eu sou hoje, e os que me amam e me apoiam incondicionalmente, sempre.

Aos meus tios, Sérgio e Suzete, que me acolheram por tanto tempo desde que vim morar em São Paulo, e acompanharam grande parte de minha trajetória acadêmica, sempre me amparando e me motivando.

À todos os meus amigos que acompanham meus dramas da vida acadêmica e que anseiam pela conclusão deste doutorado, mais do que eu mesma.

Por fim, sou eternamente grata à quem está ao meu lado todos os dias, acompanhando de perto minha evolução como pesquisadora e a própria evolução deste trabalho - o Gabriel, meu companheiro e parceiro de vida há tantos anos. Pelo seu amor, paciência e suporte incondicionais. 


\section{Abstract}

The pathways which compose the upper limb of the Global Overturning Circulation are investigated in the South Atlantic sector. These are comprised by a northward advection of waters starting at the southeastern corner of the basin which brings Pacific and Indian Ocean contributions through the 1) Benguela Current - 2) South Equatorial Current - 3) North Brazil Undercurrent system all the way into the Northern Hemisphere and its subpolar region in order to compensate the process of deepwater formation. The spatio-temporal evolution of the flow is addressed, with focus on the low-frequency variability and response to climate change. Assuming that the South Atlantic subtropical gyre (SASG) and the upper limb of the Atlantic Meridional Overturning Circulation (AMOC) are coupled systems, the interplay between these large-scale oceanic features is explored. This is accomplished through three independent investigations. First, CESM1POP2 simulation results point to an intensification and southward displacement of the SASG circulation system from the 20th into the 21 st century, associated with increased equatorward transport at the western boundary, along the AMOC upper limb. Secondly, subtropical western South Atlantic circulation changes between natural climate extremes of the Last Millennium are addressed with the CESM1-Last Millennium Ensemble Project, revealing a weaker (stronger) anticyclonic SASG circulation during the warm (cold) period, with decreased (increased) northward transport associated with the AMOC upper limb. Lastly, the forced response of South Atlantic pathways which are linked to the AMOC and the SASG are illustrated in historical simulations and future projections, according to a pessimistic radiative forcing scenario (the RCP8.5), by the 40-member ensemble mean of the CESM1-Large Ensemble. Up- to downstream long-term trends are found, linked to an observed and projected AMOC weakening as well as to windforced upper ocean circulation changes, in accordance with the recent scientific literature. Considering the primary research findings of these three investigations, this thesis provides important contributions to the understanding of the timeevolving dynamics of fundamental circulation systems in view of unprecedented human-induced climate change.

Keywords: South Atlantic; AMOC upper limb; South Atlantic subtropical gyre; climate change. 


\section{Resumo}

Os caminhos que compõem o ramo superior da Circulação de Revolvimento Global são investigados no setor do Atlântico Sul (AS). Estes são caracterizados por uma advecção de águas para norte, com início na borda sudeste da bacia, trazendo contribuições dos oceanos Pacífico e Índico através do sistema: 1) Corrente da Benguela - 2) Corrente Sul Equatorial - 3) Subcorrente Norte do Brasil, se estendendo até o Hemisfério Norte e sua região subpolar, para compensar o processo de formação de água profunda. A evolução espaço-temporal do fluxo é analisada, com foco na variabilidade de baixa-frequência e resposta às mudanças climáticas. Assumindo que o giro subtropical do AS (GSAS) e o ramo superior da Circulação de Revolvimento Meridional do Atlântico (CRMA) são sistemas acoplados, é explorada a interação entre essas feições oceânicas de grandeescala. Isso é realizado através de três investigações independentes. Primeiro, resultados de simulação do CESM1-POP2 apontam para uma intensificação e deslocamento para sul do sistema de circulação do GSAS no período de transição entre os séculos 20-21, associados com um aumento do transporte em direção ao equador na borda oeste, ao longo do ramo superior da CRMA. Em seguida, mudanças na circulação da porção oeste do AS subtropical entre extremos climáticos do Último Milênio são investigadas com dados do CESM1-LME, revelando uma circulação anticiclônica do GSAS mais (menos) intensa durante o período quente (frio), com diminuição (aumento) do transporte para norte associado com a CRMA. Por fim, a resposta forçada dos caminhos de circulação do AS que estão ligados à CRMA e ao GSAS é representada através de simulações históricas e projeções futuras, de acordo com um cenário pessimista de forçante radiativa (o RCP8.5), pela média dos 40 membros do conjunto CESM1-LE. Tendências de longo prazo são encontradas desde porções anteriores a posteriores ao longo do fluxo, as quais estão associadas ao enfraquecimento da CRMA observado no presente e projetado para o futuro, bem como à mudanças na circulação do oceano superior forçadas pelo vento, de acordo com a literatura científica recente. Em conclusão, esta tese de doutorado fornece contribuições importantes para o entendimento da evolução temporal da dinâmica de sistemas de circulação fundamentais no contexto atual de mudanças climáticas antropogênicas sem precedentes.

Palavras-chave: Atlântico Sul; ramo superior da CRMA; giro subtropical do Atlântico Sul; mudanças climáticas. 


\section{List of Figures}

1 The Great Ocean Conveyor Belt. . . . . . . . . . . . . . . . . . . 12

2 Zonally averaged global overturning streamfunction . . . . . . 13

3 2-D schematic of the Global Overturning Circulation . . . . . . 15

4 3-D schematic of the overturning circulation from a Southern Ocean perspective . . . . . . . . . . . . . . . . . . 16

5 Detailed Atlantic Meridional Overturning Circulation pathways . . . 23

$6 \quad$ Large-scale South Atlantic dynamical fields. . . . . . . . . . . . . 35

$7 \quad 21$ st century transition of South Atlantic zonally averaged fields. . . 37

8 21st century intensification of the SASG circulation. . . . . . . . 38

9 Intensity and latitude of maximum zonally averaged fields. . . . . 39

10 SASG poleward displacement and core intensification. . . . . . . . 40

11 Monitoring the position of SASG dynamical boundaries. . . . . . . 41

12 Long-term change of the SBL. . . . . . . . . . . . . . . . . 44

13 Climatological flow field (1970-2015). . . . . . . . . . . . . . 46

14 Boundary current transports and MOC time series. . . . . . . . . 47

15 Sea surface temperature anomalies $\left({ }^{\circ} \mathrm{C}\right)$ with respect to the LM. . 56

16 LM-mean SAO circulation pattern. . . . . . . . . . . . . . . 58

17 MCA and LIA anomalous SAO circulation pattern. . . . . . . . 60

18 SBL and total SSEC time series. . . . . . . . . . . . . . 61

19 Time series of horizontal volume transport anomalies. . . . . . . . 64

20 Wind stress curl anomalies with respect to the LM . . . . . . 66

21 Horizontal velocities - mean and trends . . . . . . . . . . . 81

22 Southern eastward flow prior to the Drake Passage. . . . . . . . 87

23 Southern eastward flow through the eastern South Atlantic sector. 88

24 Southern eastward flow entering the Indian Ocean sector. . . . . 89

25 Vertical profiles and corresponding volume transport time series from the Drake Passage into the South Atlantic . . . . . . . . . . . 94

26 Vertical profiles and corresponding volume transport time series from the Agulhas Leakage towards the South Atlantic western boundary and downstream beyond . . . . . . . . . . . . . . 98 
27 Transbasin meridional velocity profiles and the evolution of their zonally integrated anomalies . . . . . . . . . . . . . . . . . 100

28 SASG-core $\left(30^{\circ}-10^{\circ} \mathrm{W}\right)$ structural changes . . . . . . . . . . . . . . 103

29 Poleward shift of the southwestern SASG boundary . . . . . . . . 105

30 Equatorward shift of the sSEC bifurcation latitude at the northwestern boundary of the SASG . . . . . . . . . . . . . . . . 106

31 Changes in the distribution of zonal and meridional SASG flows . 108

32 SSH mean field and anomalous 1920-2100 total trends . . . . . . 110

33 Surface SBL time series from CESM1 simulations - the ocean-only

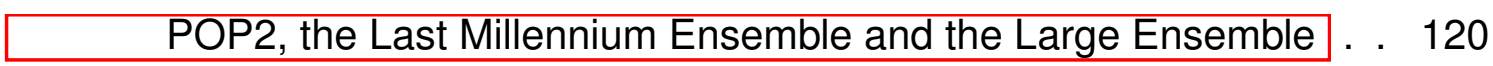

34 Comparative time range of each model simulation shown in Figure 33 . . . . . . . . . . . . . . . . . . . . 121

35 Atlantic sector of the Southern Ocean topography centered at the Weddell Sea. . . . . . . . . . . . . . . . . . . . . . . . . . . 127

36 Global sea level trends from model and observations . . . . . . . . 129

37 Changes in volume transports around Antarctica . . . . . . . . . . 130

38 Leading EOF of non-detrended zonal velocity anomalies within the SASG . . . . . . . . . . . . . . . . . . 169 


\section{List of Tables}

1 Selected time period, horizontal resolution and number of vertical

layers above 1000-m of model simulations and ocean reanalysis
products. . . . . . . . . . . . . . . . . . 28

2 Increased WSC, SSH and BSF values within contours . . . . . . . 38

3 Linear trends from the time series in Figure 9 . . . . . . . . . . . . 40

4 1970-2015 total shift in the time series from Figure 11 ]. . . . . . . 42

5 LM-mean and standard deviation; and MCA/LIA anomalies. . . . . 65

6 Two-sided $t$-test values between the MCA/LIA and the LM . . . . . 65 


\section{List of Abbreviations}

AABW Antarctic Bottom Water

ACC Antarctic Circumpolar Current

AC Agulhas Current

AL Agulhas leakage

AMOC Atlantic Meridional Overturning Circulation

ARC Agulhas Return Current

BC Brazil Current

BeC Benguela Current

BMC Brazil-Malvinas Confluence

CESM1 Community Earth System Model version 1

DP Drake Passage

DWBCs deep western boundary currents

GOC Global Overturning Circulation

GS Gulf Stream

IPCC Intergovernmental Panel on Climate Change

ITCZ Intertropical Convergence Zone

LMM Last millennium

MC Malvinas Current

MOC Meridional Overturning Circulation

NAC North Atlantic Current

NADW North Atlantic Deep Water 
NBUC North Brazil Undercurrent

NH Northern Hemisphere

PC Principal Component

POP2 Parallel Ocean Program version 2

RCP Representative Concentration Pathway

SAC South Atlantic Current

SAO South Atlantic Ocean

SASG South Atlantic subtropical gyre

SPSG South Pacific subtropical gyre

SEC South Equatorial Current

SSEC southern branch of the South Equatorial Current

SBL SSEC bifurcation latitude

SH Southern Hemisphere

SSPS Shared Socioeconomic Pathways

TF Tasman Front

WBCs western boundary currents 


\section{Introduction}

\subsection{Preamble and Thesis Outline}

This thesis presents results that concern the investigation of Past to Future South Atlantic Overturning Circulation, considering its upper-ocean pathways and low-frequency variability. This is accomplished through three different investigations (Chapters 3, 4 and 5) that each explore specific spatio-temporal perspectives of the South Atlantic Ocean (SAO) large-scale circulation, encompassing both its wind-driven component - represented by the South Atlantic subtropical gyre (SASG) - and the buoyancy-driven component - the upper limb of the Atlantic Meridional Overturning Circulation (AMOC).

Wind and buoyancy forcings interact in non-linear ways within the SAO basin, strongly influencing both these circulation features - the SASG and the AMOC upper limb. Therefore, variations in the anticyclonic circulation of the subtropical gyre may reflect variations in the upper limb flow structure, and viceversa. The SAO provides the connection between the North Atlantic and the Indian, Pacific and Southern Oceans; and its circulations reflect variations in the global wind field - mainly associated with the wind stress curl influence on the midlatitude Southern Ocean supergyre - as well as in thermohaline forcings, mainly associated with the process of high-latitude deep water formation.

For this reason, this thesis focuses on investigating SAO circulation pathways through state-of-the-art model simulation results, considering the interplay between the coupled < SASG and AMOC upper limb > features, and highlighting the importance of understanding up-to-downstream propagation of upper-ocean waters through the SAO basin in the context of the Global Overturning Circulation (GOC).

Essentially, it is desired to understand how SASG and AMOC upper limb dynamics functioned in past extreme climates and how they might respond to recent and future human-induced climate change.

To this end, Section 1.2 provides a comprehensive review of the GOC and its basic dynamics, leading to its central stage - the Atlantic Ocean, and the AMOC; to finally put in context the role played by the SAO and its large scale 
circulations. Sections 1.3 and 1.4 pose the formal guidelines framing this thesis: the Motivation and Research Questions, and the Objectives, respectively.

Chapter 2 presents all datasets used in Chapters 3, 4 and 5, including model simulations and ocean reanalysis products. A brief discussion on the relevant characteristics defining the main simulation results is given.

Chapter 3 addresses changes in the whole SASG circulation and the bifurcation of the southern branch of the South Equatorial Current (SSEC) during the period of 1970-2015 using simulation results from the ocean component of the Community Earth System Model version 1 (CESM1) - the Parallel Ocean Program version 2 (POP2) (Marcello et al., 2018).

Chapter 4 explores more specifically the South Atlantic surface boundary current system, off the South American coast, investigating the north-south redistribution of the westward flow after the SSEC bifurcation during contrasting climatic periods of the last millennium: the warm Medieval Climate Anomaly (MCA) and the cold Little Ice Age (LIA). Simulation results from the Community Earth System Model Last Millennium Ensemble (CESM-LME) are used (Marcello et al., 2019).

While Chapters 3 and 4 respectively highlight changes in the SASG circulation and its spatial structure, and relative changes in the apportion of waters between the SASG and the AMOC upper limb during past extreme warm and cold periods, Chapter 5 gives focus to the South Atlantic portion of the AMOC upper limb, encompassing the Pacific and Indian Ocean contributions to the flow as well as their incorporation to the SASG circulation prior to proceeding the northward advection and crossing the equator. The low-frequency historical variations (19202005) and projected changes (2005-2100) of the AMOC upper limb pathways are investigated using 40 members of the Community Earth System Model Large Ensemble Project (CESM1-LE), a tool that enables the estimation of uncertainty in recent and future climate due to the presence of internal climate variability (Marcello et al., in prep.).

Other publication results developed in collaborations along the Doctoral Postgraduate Program are summarized in Chapter 8 . A list of all publications is presented on Section 9.

In terms of discussions with respect to last scientific advances contex- 
tualized in space and time, the "Introductory remarks" in Chapter 3 focuses on recently reported changes in global subtropical gyres in response to shifted wind-patterns, in Chapter 4 it argues on past millennium global scale climate variability with focus on the respective warm and cold climate extremes, and in Chapter 5 it provides a general review on the current state of knowledge regarding the present AMOC state and its future prediction, also considering updates on wind-driven global circulation changes. 


\subsection{Theoretical Background}

This section is intended to provide a broader overview, introducing the concept of the ocean's Global Overturning Circulation as an important component of the climate system and describing its overall functioning, which has been increasingly understood and elucidated mainly in the past decades. Then, focus is given to its Atlantic sector (represented by the AMOC) and, particularly, to the upper-ocean return limb of the overturning circulation back to the subpolar North Atlantic (the AMOC upper limb). Ultimately, it is put in perspective the fundamental role played by the South Atlantic through enabling interocean connections and the means for upper limb waters to propagate northward, embedded in its large scale circulations - thus providing the context necessary for the reader to interpret and associate the results presented in Chapters 3, 4, and 5.

\subsubsection{The Global Overturning Circulation and the Climate System}

The Earth's climate system is primarily driven by energy from the sun in form of radiation that is unevenly received across Earth's surface. The heat transport that is accomplished by the atmosphere and ocean acts to even out solar heating imbalances and reduce equator-pole temperature gradients (e.g., Kump et al., 2004).

Looking more closely into this process, the effect of the sun's radiation together with Earth's rotation on the atmosphere generates pressure gradients and therefore the winds; and the wind stress in turn applies momentum to the ocean's surface, giving rise to the upper-ocean wind-driven circulation. On the other side, differing heating and cooling caused by the sun in Earth's atmosphere also imply differences in the ocean's temperature, the same way that atmospheric evaporation and precipitation force differences in the ocean's salinity - which together result in density variations that force the deep ocean's thermohaline (or buoyancy-driven) circulation (Marshall and Plumb, 2016; Wells, 2012).

And, finally, this is how the Global Overturning Circulation (GOC - Talley, 2013; Cessi, 2019) is conceived: as the large-scale movement of the global ocean that includes both the wind-driven circulation and thermohaline-induced fluxes, connecting the flow in the upper layers of the ocean to the flow in the deeper layers 
through the processes of convection at high latitudes and localized wind-driven as well as slow diffusive upwelling elsewhere (Drijfhout et al., 2013; Rahmstorf, 2006; Schmittner et al., 2007; Wunsch, 2002).

Simply put, the GOC can be viewed as the global-scale motion of the ocean. It fairly represents the ocean's fundamental role in the climate system, functioning as a great conveyor belt that circulates and redistributes large amounts of heat and freshwater along with nutrients and other important tracers such as carbon and oxygen around the globe.

Yet, let us take a step back to a bigger perspective, in order to better elucidate the contrast between ocean and atmosphere properties and their particular performance in maintaining the climate system, and also to further illustrate the GOC's relevance to the global climate. The density contrast between air and water means that the mass of the ocean is a lot greater than that of the atmosphere (up to 270 times), which, together with water's high specific heat capacity, implies in the ocean's large heat capacity (4 times that of the atmosphere - meaning that only $\sim 2.5 \mathrm{~m}$ of ocean water has the same heat capacity as the whole atmosphere). That is of notorious importance for seasonal changes as well as for climatic variations at longer timescales - once the ocean acts as a stabilizer thermal-reservoir, storing the excess heat and slowly releasing it over long periods of time (Gill, 2016; Marshall and Plumb, 2016).

Not to mention the great capacity that cold surface ocean waters have to absorb carbon dioxide $\left(\mathrm{CO}_{2}\right)$ from the atmosphere (Weiss, 1974): the ocean typically stores over 50 times more carbon than the atmosphere (once $\mathrm{CO}_{2}$ reacts with sea-water forming dissolved inorganic carbon); and only the 100-m surface mixed layer of the ocean holds a comparable amount of carbon as that stored in the whole atmosphere. For this reason, minor changes in the ocean's reservoir of carbon can lead to large changes in atmospheric $\mathrm{CO}_{2}$ (Williams and Follows, 2011). Currently, the ocean is a sink for at least $25 \%$ of the atmospheric $\mathrm{CO}_{2}$ emitted by human activities (Friedlingstein et al., 2019; Watson et al., 2020). Without the ocean's carbon uptake, atmospheric levels of $\mathrm{CO}_{2}$ would now be close to 600 parts per million (ppm), almost 50\% higher than the 410 ppm recorded in 2019 (Aricò et al., 2021). 
In short, the ocean is a giant reservoir for both the excess heat and carbon, globally. More than 30\% of the total $\mathrm{CO}_{2}$ (Gruber et al., 2019) and $90 \%$ of the heat (Ciais et al., 2014) added to the atmosphere by human activities since the beginning of the industrial revolution have been taken up by the oceans.

But these compensation processes are not all passively accomplished by waters just standing out there in the ocean. They are actually due, largely, to the GOC system - the three-dimensionally moving waters across the global ocean. Excess heat in the atmosphere is uptaken and stored in the ocean by mechanisms that are part of the overturning circulation: mainly the wind-driven upwelling of cold deep waters in the Southern Ocean that takes up and carries the heat content anomalies northward and downward into the thermocline, also limiting the surface warming at the uptake region therefore allowing the heat uptake to be sustained (Morrison et al., 2016); and the formation of deep water in the subpolar North Atlantic, where strong heat loss occurs from the ocean to the atmosphere (which is analogous to heat uptake from the atmosphere to the ocean) (Shi et al., 2018).

Likewise, oceanic $\mathrm{CO}_{2}$ uptake happens directly via meridional transport and overturning (Pérez et al., 2013): as anthropogenic carbon is effectively circulated and carried north of the North Atlantic subtropical gyre (Rosón et al., 2003), for instance; and also as these northward-flowing surface waters cool and absorb additional $\mathrm{CO}_{2}$ that is carried to depth when the deep waters form in the subpolar North Atlantic (Steinfeldt et al., 2009). The oceanic $\mathrm{CO}_{2}$ uptake can also be indirectly influenced by the overturning's variability through its effect on ocean's primary productivity, in case there's a disruption either in the northward transport or in the mixing and convective surface supply of nutrients (Palter and Lozier, 2008).

All the above illustrates how the ocean plays an essential role in the climate system - especially amidst the current global warming scenario - and how the GOC engine is greatly responsible and representative of this role. To understand how the ocean affects the global climate, mainly through $\mathrm{CO}_{2}$ uptake and warming, one needs to understand how the ocean circulates, redistributing carbon and heat. Ocean circulation changes have the potential to alter Earth's heat budget, especially if certain tipping points are crossed - after which the climate 
system can quickly shift away from its current state into a contrasting, alternative state (Heinze et al., 2021; Scheffer et al., 2001).

In fact, changes in Earth's heat budget, arising either from natural variations or human-induced global warming, can trigger responses in the climate system by forcing a trajectory "for which a slight deviation above a critical value that continues for some time inevitably induces a qualitative change" (Lenton et al., 2008). There may be no abruptness, but still a qualitative impact in the future (Ritchie et al., 2021). The ocean's GOC is considered a key tipping element of the climate system, with the potential to trigger global impacts (Barker and Knorr, 2016).

With such a profound array of implications, it is no surprise that a mechanistic understanding of the GOC, its processes and variability is currently a high priority for the climate science community (Lozier et al., 2017). After all, despite many uncertainties still concerning time and space, it is long known that the ongoing increase in atmospheric $\mathrm{CO}_{2}$ levels might trigger abrupt changes and reorganizations in the ocean's overturning circulation, considering its ability to assume more than one mode of operation, according to paleorecords that reveal evidence pieces of GOC's deep past (Broecker, 1997; Rahmstorf, 2000, 2002). This possibility has not been ruled out ever since, and it is actually a recent topic of great concern (Weijer et al., 2019).

The interconnectedness between the climate system components allows energy to continuously flow through the atmosphere-ocean/hydrospherecryosphere-lithosphere-biosphere, while the key processes governing the functioning and dynamics in each of these five spheres dictate how the energy is exchanged among them across a wide range of timescales. Besides being considered a key process governing the ocean's circulation, the GOC also directly influences all of the other climate components/spheres to some degree.

\section{Key concepts}

It is worth noting that the GOC term is more adequate to refer to vertical as well as both zonal and meridional flows in the ocean. The term Meridional Overturning Circulation (MOC), however, reflects the latitudinal volume trans- 
port associated with the two dominant, global-scale, counterrotating meridional circulation cells (Lumpkin and Speer, 2007).

Even though the overturning circulation is, in general, promptly associated with the vertical-meridional plane, its zonal components are also crucial, especially when it comes to inter-basin transports and oceanic gyres (Talley, 2011). Still, the most intriguing aspect of the overturning circulation is that of the meridional component, linked to the vertical fluxes that allow transformation of upper, lighter waters in deep and bottom denser waters and vice-versa.

For this reason, the term MOC is more widely recognized and often used interchangeably with the term GOC, even though they have distinctly different meanings. Hereafter, the term MOC will be predominantly used, in correspondence with the majority of the literature, assuming the reader bears in mind its literal meaning and broad application. The MOC's mathematical definition is given at the end of this section (1.2.1.

\section{MOC's history and description}

The existence of the ocean's MOC, or some idea of it, was first supposed by Count Rumford, in the year of 1800 , after a single measurement of deep-ocean temperatures revealing "icy-cold" abyssal waters led him to suspect that highlatitude surface waters tended to lose their heat to the surrounding cold winds, therefore getting denser and sinking to the ocean floor - from where these waters would spread towards the equator, subsequently "necessitating a current at the surface in an opposite direction" (Warren, 1981).

A century later, Schott (1902) proposed the idea of two symmetric overturning circulation cells, located on either side of the equator in the Atlantic Ocean.

During 1947-1950, Harald Sverdrup, Henry Stommel and Walter Munk developed their foundational theories on the wind-driven circulation: (i) relating the curl of the wind stress to the circulation in the upper $\sim 1000 \mathrm{~m}$ of the ocean, with the Coriolis forcing varying with latitude (Sverdrup, 1947); (ii) considering bottom friction (besides only the wind friction) and revealing the existent asymmetry in oceanic gyres due to their westward intensification (Stommel, 1948), (iii) and then complementing with also lateral eddy friction to enclose the picture of a more 
realistic wind-driven circulation (Munk, 1950).

Later on, Stommel also theorized that high-latitude deep waters were transported equatorward along deep western-intensified boundary currents, such as in the surface circulation, but in the opposite direction (Stommel, 1958) - finally giving the ocean's overturning a third-dimensional structure. He also disproved Schott's idea, emerging with globally connected deep western boundary currents (DWBCs), linked across hemispheres and basins.

Stommel revolutionized the concept of the overturning circulation, by ultimately providing a schematic of a continuous conveyor-belt-like MOC: (i) connecting sinking of northern surface waters to upwelling of deep waters throughout the oceans (also explaining how the high gradient of the ocean's thermocline is maintained); (ii) showing the deep water movement towards its source through the western boundary; (iii) including the Indian and Pacific basins through an interconnected deep flow of the global oceans (where deep waters formed in the North Atlantic and Weddell Sea feed these basins from the south, since they don't have deep-water formation sites); (iv) assuming some of the deep flow to follow the Antarctic Circumpolar Current around the globe back to the Atlantic; and, finally, (v) merging the wind-driven with the deep, "internal" ocean circulation (Stommel, 1957, 1958, 1965). Furthermore, the possibility that the MOC has more than one stable mode, arising from the competing thermal and haline circulation effects, was first postulated in Stommel (1961).

Stommel only did not create a schematic of the upper layer return flow of the MOC - although it was obvious that the water upwelled in the Pacific and Indian Oceans must return to the Atlantic around either southern Africa or South America or even both (Richardson, 2008). A couple decades later, Gordon (1985, 1986) specified the routes and transports of the return flow from the Pacific and Indian Oceans for the first time, assuming that the warm water route from the Indian Ocean Agulhas Leakage was dominant over the cold-water route from the Pacific Drake Passage flow.

Although Stommel's work provided the MOC's spatial structure, its temporal context and recognition as an agent of climate change was effectively brought about by Wallace S. Broecker, during the 80 's. He is the one who came with the 
famous term "Global Conveyor Belt Circulation" or "Great Ocean Conveyor Belt" - which referred to the MOC as a closed loop in a simplified conceptual model. It is worth noting that, during that time, this term and the term "Thermohaline Circulation" (THC) were widely used; only in the early 21 st century the term MOC was recognized as the most suitable one, since it is a merely descriptive, "driverneutral" term (defined by a circulation in the meridional-vertical plane - Kuhlbrodt et al., 2007); while the THC term is actually a definition of the flow by only one of its driving mechanisms; and the conveyor belt is only a simplistic concept that neglects various important aspects of the ocean's overturning circulation.

Broecker et al. (1985) proposed interactions between climate and ocean circulation through feedback loops that may amplify climatic change; and linked "several brief climate oscillations during glacial time" to "fluctuations in the formation rate of deep water in the northern Atlantic".

In this study, Broecker et al. (1985) state that although orbital forcing is widely accepted as the primary cause of glacial cycles - with non-linearities in the response of snow and ice cover to seasonal variations driving glacial growth and retreat - it is not clear whether this mechanism alone is sufficient to account for the whole of these asymmetric climate cycles, which involved long periods of glacial buildup suddenly terminated with rapid warmings. It was then raised the possibility that these brief warming events, recorded in ice cores, could represent periods during which "the glacially weakened northern Atlantic deep-water source was rejuvenated" (once during peak glacial times the deep-water production was greatly reduced).

During these years, Broecker began to call attention for the fact that human-induced global warming was pushing the Earth into an unknown realm, in which "we can't predict how the great ocean conveyor belt will respond", nor the other important elements of the climate system. "We, the inhabitants of planet Earth, are performing a gigantic climate experiment." - he said in his article entitled "The biggest chill" (Broecker, 1987a), referring to the release and buildup of greenhouse gases in the atmosphere, by fossil-fuel burning. Concerns started about the possibility of high-latitude large meltwater discharges coming to disrupt the process of deep-water formation and, consequently, the MOC — which would 
then cause further changes in atmospheric greenhouse gas content and albedo. It was known about the connection between changes in atmospheric $\mathrm{CO}_{2}$ content and glacial-interglacial temperatures, and it was suspected that the origins of past changes in atmospheric $\mathrm{CO}_{2}$ content lied in the oceans (Broecker and Denton, 1990).

Several subsequent works by Broecker kept on stressing the dangers of humans releasing heat-trapping gases into the atmosphere, and emphasizing the possible impacts to ocean circulation patterns and intensity - peril of leading to important reorganizations and even a "shut-down of the conveyor belt". Along these works, the conceptual picture of the "Great Ocean Conveyor Belt", considered a metaphor for the real overturning circulation (Richardson, 2008), took form and was rapidly popularized (Figure 1): "Unpleasant surprises in the greenhouse?" (Broecker, 1987b), "The role of the ocean-atmosphere reorganizations in glacial cycles" (Broecker and Denton, 1990), "The Great Ocean Conveyor" (Broecker, 1991), "Thermohaline Circulation, the Achilles Heel of Our Climate System: Will Man-Made $\mathrm{CO}_{2}$ Upset the Current Balance?" (Broecker, 1997), "What if the Conveyor were to shut down? Reflections on a possible outcome of the Great Global Experiment" (Broecker, 1999).

The conveyor belt is obviously a gross oversimplification of the real MOC, only loosely defined as flow that carries heat and salt from high to low latitudes and vice versa (Wunsch, 2002); it only expresses the unifying essence of the global overturning. It omits fundamental features and characteristics of the circulation, for example: the horizontal flow fields, ocean gyres, western boundary currents, the circumpolar current and the flow through the Drake Passage. It also implies steady currents, while the ocean actually has strong fluctuations at different frequencies (Richardson, 2008).

From the beginning of the 21 st century to the present, a number of studies have increasingly revealed the true complexity of the global MOC system, both in terms of its structure and dynamics - as the ocean has increasingly been observed at finer scales in space and time and in places previously only sparsely sampled.

Initially, some light was shed on the vital role of the ocean's eddy and wind fields in establishing the realistic MOC structure and variability (Wunsch and 


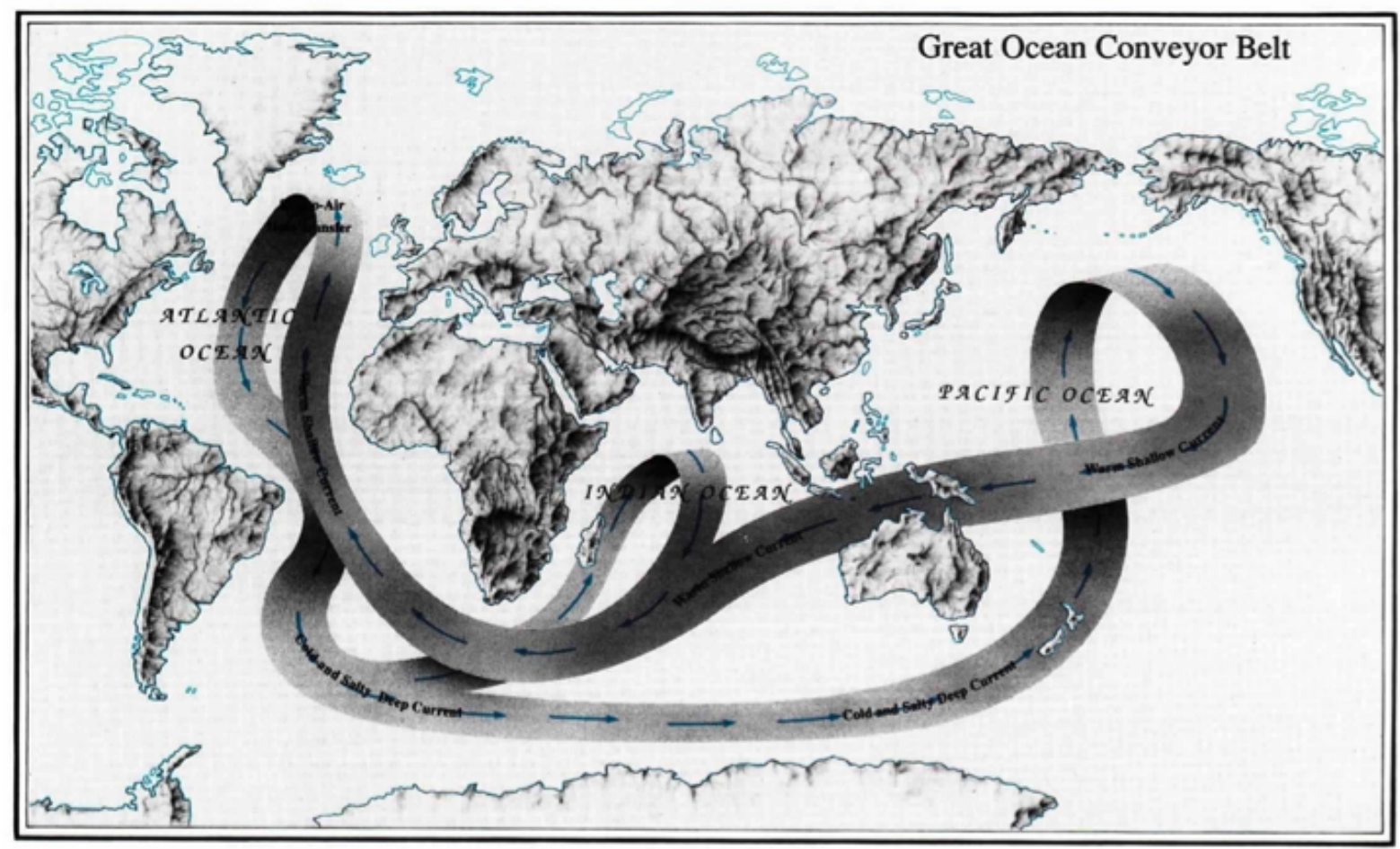

Fig. 1: The great ocean conveyor logo (Broecker, 1987). (Illustration by Joe Le Monnier, Natural History Magazine.)

Figure 1 The Great Ocean Conveyor Belt. Extracted from Broecker (1991).

Ferrari, 2004). Likewise, the notion that Southern Ocean processes are pivotal for setting the mean strength of the overturning circulation has emerged (Toggweiler and Samuels, 1995; Marshall and Speer, 2012).

Up to now, new interior pathways and routes have continued to be discovered and further elucidated (e.g., Bower et al., 2009; Lozier et al., 2013; Garzoli et al. 2015; Bower et al., 2019). A series of comprehensive reviews punctuating recent advances and the present scientific understanding of the overturning circulation have been published in the last couple years. Theoretical aspects are reviewed by Johnson et al. (2019), a synthesis on the MOC's stability is provided by Weijer et al. (2019), the effect of mesoscale eddies as depicted by high-resolution models is discussed by Hirschi et al. (2020), among other reviews from different aspects of the MOC (which are mostly summarized by Srokosz et al., 2021).

Today, the ocean's overturning circulation is generally perceived as a global cell formed by a system of horizontal and vertical currents driven by wind, by buoyancy forcing through evaporation, precipitation, heating and cooling and also by mixing due to winds and tides. It encompasses all ocean basins, connecting the 
surface ocean and atmosphere to the deep sea (Lozier, 2010; Schmittner et al., 2007).

More specifically, this global overturning cell is formed by two dominant interconnected cells, centered in the Atlantic Ocean: a mid-depth clockwise cell, associated with the southward transport of North Atlantic Deep Water (NADW) at depths between $\sim 1-3 \mathrm{~km}$ along the lower limb; and an abyssal counterclockwise cell, linked to the northward transport of Antarctic Bottom Water (AABW) below depths of $\sim 4,5 \mathrm{~km}$ at the lower limb - which gradually mixes into the lower parts of the overlying NADW, eventually returning southward (Talley, 2013) (Figure 2).

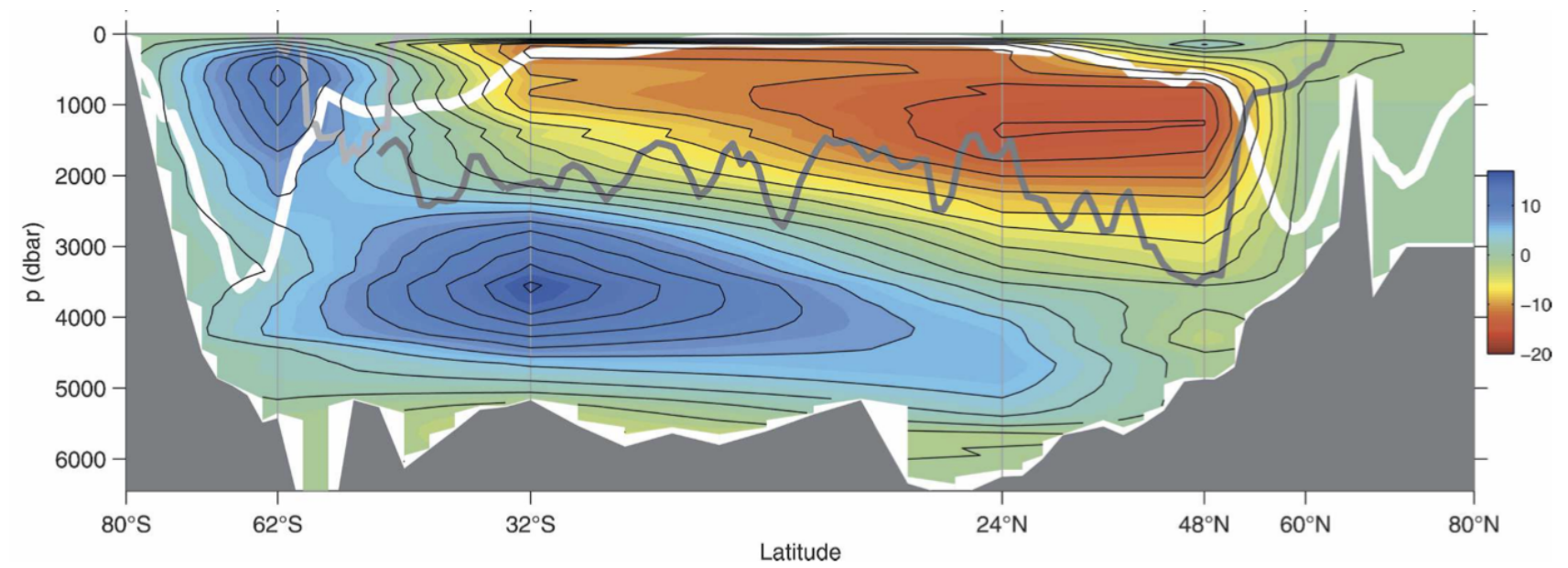

Figure 2 Zonally averaged global overturning streamfunction (Sv, contours every 2 Sv) in pressure levels across hydrographic sections (vertical gray lines) with linear interpolation between the sections. The white line represents typical winter mixed layer depths, the dark gray line and contour represents the mean depth of ocean ridge crests and the light gray line at the upper-left portion of the domain represents the depth of the Scotia Arc east of the Drake Passage. Extracted from Lumpkin and Speer (2007).

A simple excursion along the main MOC pathways, taking the North Atlantic polar region as the starting point, begins:

(i) with the formation of NADW by strong cooling resulting in buoyancy loss and sinking;

(ii) the NADW then flows away from its source region and across the equator mainly through the Deep Western Boundary Current (DWBC) and complex interior pathways (Lozier, 2012; Garzoli et al., 2015);

(iii) by reaching the Southern Ocean, part of the NADW is further densified by cooling and brine rejection in the Artarctic forming AABW which is then spread via the Antarctic Circumpolar Current (ACC) and globally connected DWBCs 
towards the Pacific and Indian basins;

(iv) deep waters rise to the surface through diapycnal upwelling (turbulent mixing across surfaces of equal density) occurring in the interior of the Indo-Pacific basin and also through Southern Ocean, adiabatic, wind-driven upwelling (through the "Drake Passage Effect" - in which near-surface waters pushed northward by the Ekman transport can only be replaced by deep upwelled waters due to the lack of topographic barriers at this latitude band, fulfilling a continuity requirement Toggweiler and Samuels, 1995; Rintoul et al., 2001; Rahmstorf, 2006; Kuhlbrodt et al., 2007);

(v) upper-ocean waters are then returned to the source region through the upper-limb of the MOC: Indian Ocean waters enter the South Atlantic via the Agulhas Leakage at the southern tip of Africa - the warm water route (Gordon, 1985), and Pacific Ocean waters flow directly from the ACC into the South Atlantic through the Drake Passage - the cold water route (Rintoul, 1991); these interocean waters are then advected northward back into the high latitudes of the North Atlantic, closing the loop.

The description provided is obviously an idealized one, in which we follow a preferred linear path that takes us through a nice, closed loop. The whole of the MOC is further complicated by several interactions and transformations to other intermediate water-masses along the way. For example, part of the northern sourced deep waters reaching the Southern Ocean can either follow a direct, adiabatic route in the Southern Ocean to the surface mixed layer (Marshall and Speer, 2012) or either make many circumpolar circuits around Antarctica before entering a basin, sinking or upwelling (Cessi, 2019). The pathways of the warm and cold water routes are also hypothesized to be linked and mixed to some degree through the 'Southern Hemisphere Supergyre' (Speich et al., 2007).

More detailed descriptions and discussions on the global overturning pathways are provided by Cessi (2019); Talley (2013); Marshall and Speer (2012); Lumpkin and Speer (2007) and Schmittner et al. (2007). Figure 3 provides a modern representation of the two-dimensional GOC.

Gordon (1991) has developed the first three-dimensional diagram of the global overturning, showing vertical sections through the Atlantic, Indian and Pacific 
Oceans connected with the Southern Ocean. It illustrates the complicated system of flows including the upper and lower overturning cells and their connections. An updated version of Gordon's diagram is shown in Figure 4 , from Talley (2013).

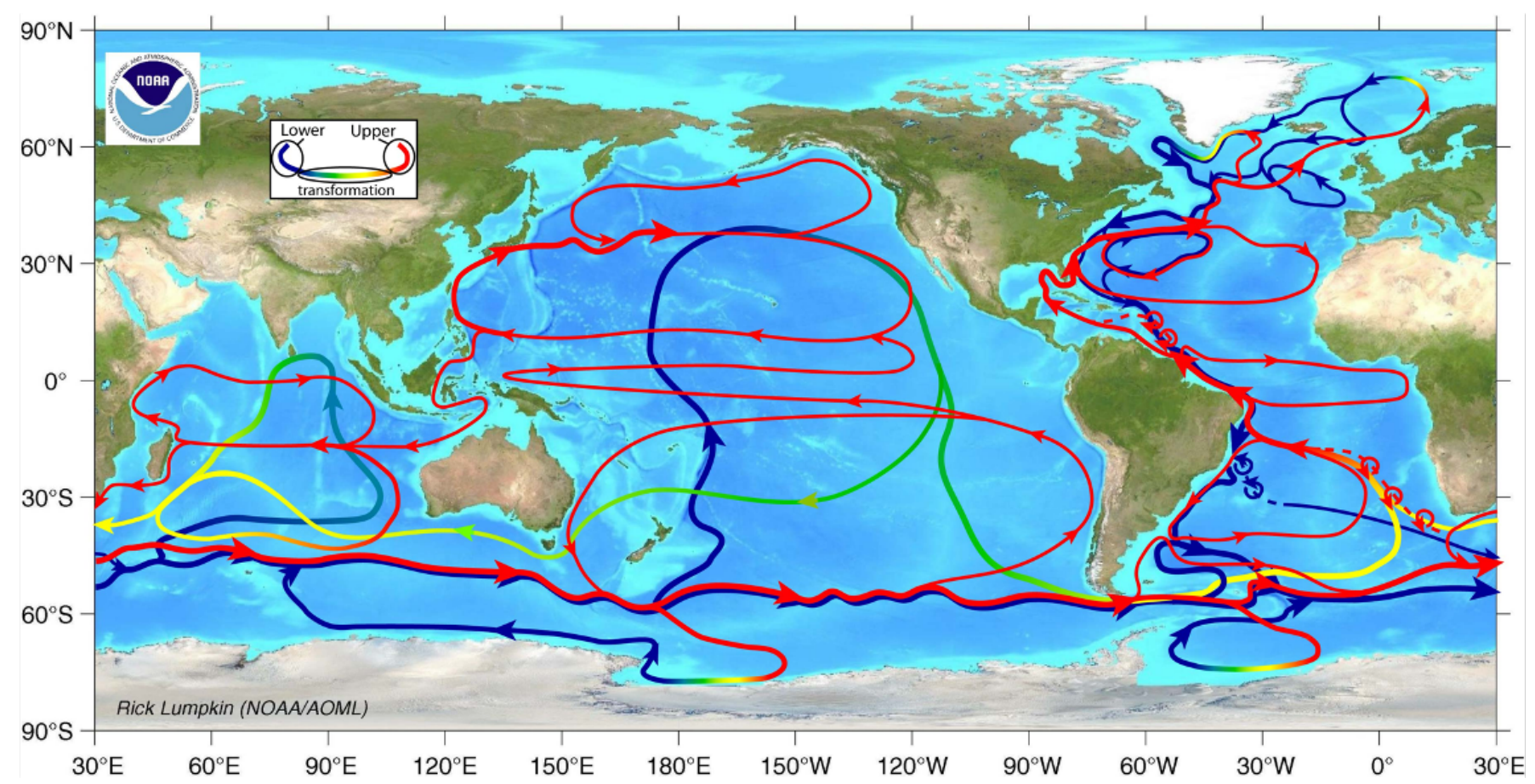

Figure 3 Two-dimensional schematic of the world ocean Meridional Overturning Circulation. Red is surface flow, blue and purple are deep flows, and yellow and green represent transitions between depths. Extracted from (Garzoli and Matano, 2011).

\section{Drivers of the overturning circulation}

Having described the main MOC branches - consisting of deep water formation, spread and upwelling, followed by a near-surface return flow — important concepts concerning these features must be addressed.

Back in the 20th century, buoyancy forcing and deep water formation at high latitudes had long been considered the main driving mechanisms for the overturning circulation. In the early 21 st century, studies started to recognize the wind forcing as a considerably important mechanism, acting on the upward MOC branch - (i) either as a direct energy input to the large scale motion, causing direct wind-driven upwelling in the Southern Ocean or (ii) by providing the diapycnal (small scale, turbulent) mixing that enables the slow return of deep waters to the surface (Kuhlbrodt et al., 2007; Lozier, 2010; Marshall and Speer, 2012).

These opposing hypothesis for the MOC forcing have been referred to 


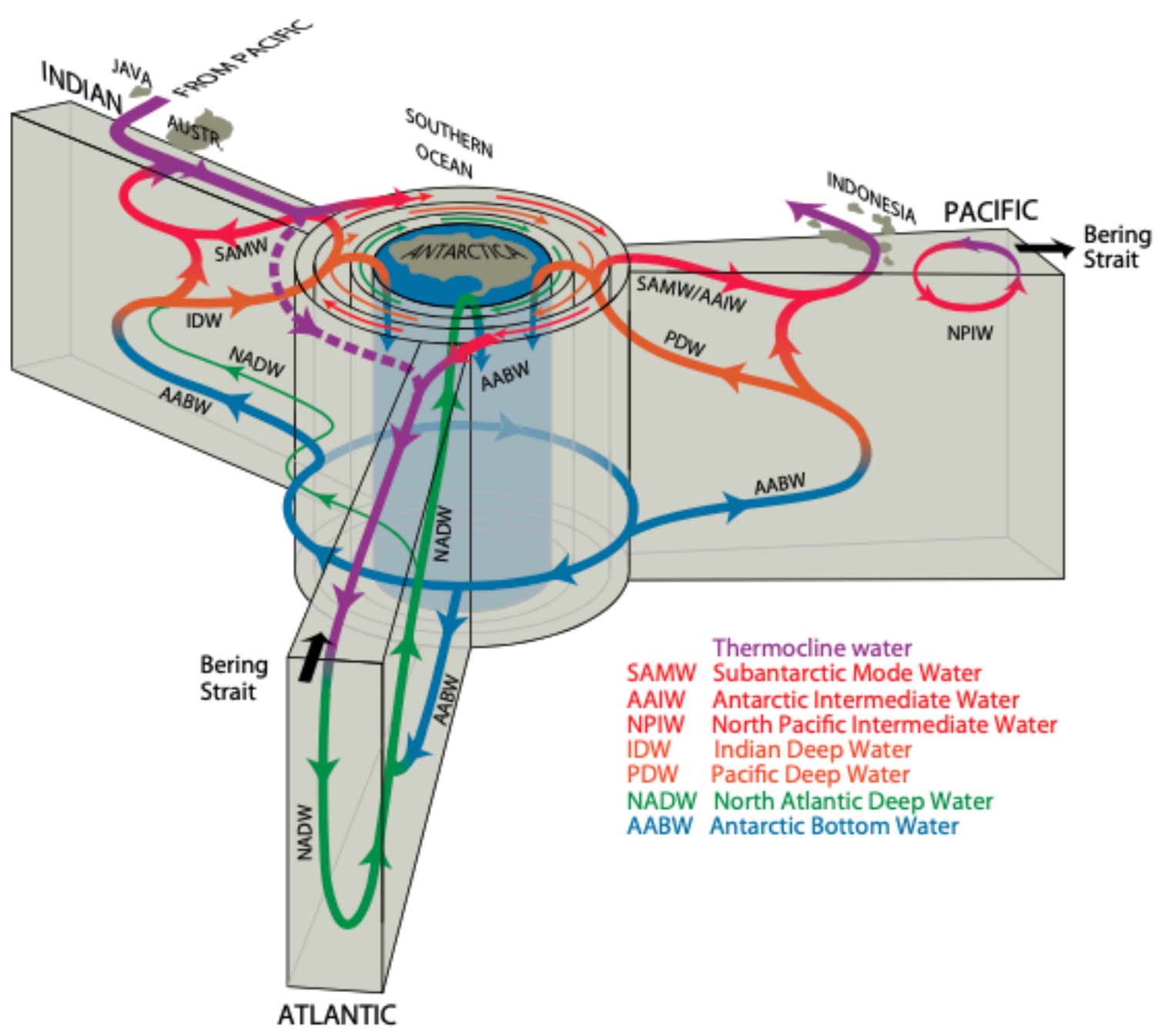

Figure 4 Three-dimensional schematic of the overturning circulation from a Southern Ocean perspective. Extracted from Talley (2013).

as "push" versus "pull" views - in which localized buoyancy loss-induced deep water formation in high latitudes "push" surface waters downward, and dissipationinduced as well as wind-induced upwelling "pull" deep waters upward (Visbeck, 2007).

Surface buoyancy forcing per se cannot sustain a steady state deep overturning, although it contributes to setting the properties of deep water masses which in turn set the interhemispheric shape and strength of the MOC, substantially influencing its transient behavior (Kuhlbrodt et al., 2007). But, instead, a deep water source is required to drive a deep overturning, which is provided by the downward penetration of heat - the turbulent diapycnal mixing that results from 
winds and tides at low latitudes (and also molecular diffusion and conduction, to some degree - Kuhlbrodt, 2008, transporting heat downward and thus raising the gravitational potential energy of the water masses (Sandström, 1908; Coman et al., 2006). Without this deep source of heat and the turbulent mixing at depth that carries dense fluid across density gradients, the deep ocean would be unstratified, with the region below the cold source eventually becoming homogeneous (Wunsch, 2002; Wunsch and Ferrari, 2004).

Therefore, the buoyancy boundary conditions do not actually drive the circulation. The buoyancy-driven flow provides the vast transport of heat and freshwater, but only as a nearly passive consequence of the mechanical energy needed to return deep waters to the surface, sourced predominantly by winds, and in second order by tides (Wunsch, 2002).

This is accomplished in part by vertical mixing of warm waters along depth, which makes deeper water less dense, overcoming stratification and resulting in upwelling - thus sustaining a density-driven ocean circulation (Visbeck, 2007). Although, it has been recently elucidated that oceanic boundary conditions such as bathymetry and topographic features dominate this mixing process, creating primarily zonal currents in the interior along sloping topography and a net meridional exchange along western boundaries - a picture which is quite different from the traditionally assumed widespread and quasi-uniform open-ocean upwelling (Callies and Ferrari, 2018).

But, more importantly, the work done by the wind field also enables the return of waters from the interior ocean to the surface through the Southern Ocean upwelling branch, which has been increasingly recognized as a vital component of the MOC and the climate system, overall (Marshall and Speer, 2012; Evans et al., 2018; Tamsitt et al., 2018; Rintoul, 2018.

Actually, it is suggested by observations that as much as $80 \%$ of global deep waters return to the surface in the Southern Ocean (Talley, 2013; Lumpkin and Speer, 2007), including both the wind-driven upwelling and the upward motion of deep water in the ocean interior, which is focused on narrow regions where eddies and bottom topography facilitate vertical motion, with small contributions of the weak diapycnal mixing over broad scales (Tamsitt et al., 2017; Rintoul, 2018). 
The remainder, less than $20 \%$, rises to the sea surface in low latitudes.

In this view, primary deep water formation in the North Atlantic unfolds simply as a sinking branch and deep outflow returning to close the loop driven by Southern Ocean surface Ekman transports. This implies that the strength of the MOC in the Atlantic basin is largely determined by the strength of the zonal wind stress component in the Southern Ocean, where stronger westerlies tend to result in an enhanced overturning circulation via enhanced northward Ekman transport (Kuhlbrodt et al., 2007).

Besides, submesoscale processes such as mixed layer instabilities and eddies are now believed to play an important role in restratifying the water column and balancing surface heat fluxes, although their role in generating low-frequency MOC variability is still under debate (Johnson et al., 2019). In fact, the Southern Ocean component of the MOC also depends strongly on the density structure of the region, which is likewise influenced by eddies, besides surface thermohaline fluxes (Kuhlbrodt et al., 2007).

Today, the paradigm of the MOC has shifted from a circulation pattern where convection-driven downwelling in the North Atlantic is balanced by diffusive upwelling in the rest of the ocean basins, to one where wind-driven upwelling and eddy-driven transports in the Southern Ocean play a dominant role (Weijer et al., 2019). In the last couple decades, "Southern Hemisphere westerlies took central stage in the theory of the deep overturning circulation - rendering obsolete the very concept of a purely thermohaline circulation" (Wunsch and Ferrari, 2018).

Previous paradigms also implied that the MOC varied on time scales of years to millennia, while now it is known that the MOC is highly variable on all time scales, from days to millennia, with much of the variability on shorter time scales being attributable to wind forcing, and the buoyancy forcing being important for longer timescales (Hirschi et al., 2020; Jackson et al., 2019; Johnson et al., 2019; Srokosz et al., 2021). These revelations have emerged together with advances in the understanding of the general ocean circulation in the past 50 years or so, revolutionized to reflect the "Era of the time-dependent ocean", as new instrumentation gradually evolved from the 1970 s onward (Wunsch and Ferrari, 2018. 
In terms of the MOC global scale structure, deep water is formed solely in the North Atlantic and not in the North Pacific simply because the Pacific is not salty enough, causing the waters to freeze before they can sink (Warren, 1983). The Atlantic's higher salinity is explained by a series of factors, including a net excess of evaporation over precipitation in the Atlantic's narrow basin and the advection of salt-rich waters from the Indian Ocean through the Agulhas Leakage (de Ruijter et al., 1999; Gordon, 1986). A set of configurations combine to create an anomalous freshwater export from the Atlantic into the Pacific basin, ultimately (Buckley and Marshall, 2016). Together, these processes are responsible for preconditioning the Atlantic for deep convection (Marsh et al., 2007). Ferreira et al. (2018) present the state of knowledge on the Atlantic-Pacific salinity difference, which is further discussed by Johnson et al. (2019).

Summing up, the MOC is overall viewed as having: (i) an active basin where downwelling primarily occurs at its northern end - the Atlantic; (ii) a passive basin that dominates diffusive upwelling - the Indo-Pacific; (iii) and a circumpolar region - pertaining to the Southern Ocean -, where wind-induced upwelling occurs and also where an interbasin connection is provided by the ACC, permitting a global overturning circulation to exist.

Therefore the MOC implies a transfer of intermediate water from the passive into the active basin, balanced by a transfer of deep water in the opposite direction (Jones and Cessi, 2016). The present study focuses on the active basin - the Atlantic Ocean - which is more comprehensively addressed hereafter.

Despite major advances have been accomplished so far in constructing a comprehensive picture of the MOC which is quite more complicated than that of the Conveyor Belt, Broecker's paradigm concerning important paleoclimatic changes in response to mode flips in the MOC was repeatedly proven to be right (Alley, 2007) and continues to be accepted until these days, providing that present climate conditions are properly accounted (Srokosz et al., 2021).

\section{MOC definition}

Formally, the MOC is identified by a mathematical definition: the zonally integrated (in an east-west section across an ocean basin) volume flux as a 
function of latitude and depth (Cunningham and Marsh, 2010).

$$
\Psi \operatorname{MOC}(y, z, t)=\int_{x_{e}, y}^{x_{w}, y} \int_{-H}^{z} v(x, y, z, t) d z d x
$$

where $\mathbf{v}(\mathbf{x}, \mathbf{y}, \mathbf{z}, \mathbf{t})$ is the meridional velocity at longitude $\mathbf{x}$, depth $\mathbf{z}$, time $\mathbf{t}$ and latitude $\mathbf{y} ;-\mathbf{H}$ and $\mathbf{z}$ are the vertical limits of integration (from a given depth to the surface) and $\mathbf{x}_{e}, \mathbf{y}$ and $\mathbf{x}_{w}, \mathbf{y}$ are the eastern and western boundaries at a given latitude.

The sum of the total poleward (or equatorward) components of this overturning yields a single measure for the strength of the meridional overturning, expressed in terms of Sverdrups ( $1 \mathrm{~Sv}=10^{6} \mathrm{~m}^{3} . \mathrm{s}^{-1}$ ); which can be estimated for individual basins or even the global ocean. Yet, this description of the MOC is a simple definition of the flow field and, as such, does not presuppose a mechanistic cause for the overturning or its variability.

\subsubsection{The Atlantic Meridional Overturning Circulation}

With the Atlantic being the active basin of the global overturning, with both the intermediate and abyssal cells centered in its sector, the majority of studies are focused on the Atlantic branch of the MOC (the AMOC); and frequently the term is even used in a generalized way, considering the AMOC itself remotely impacts processes globally. About half of the global ocean's deep waters are credited to the convection processes in the northern North Atlantic (Rhein et al., 2013).

In the Atlantic, the upper-limb return flow of the AMOC transports heat from the southern to the northern basin, characterizing a counter-intuitive net equatorward ocean heat transport in the South Atlantic (order of 0.5 PW - Dong et al., 2009; Trenberth et al., 2019) - which is a unique feature among ocean basins. At $26^{\circ} \mathrm{N}$, the approximate latitude of the Atlantic's maximum heat transport, this northward transport increases to $1.3 \mathrm{PW}$ - which is approximately $70 \%$ of the net poleward heat flux carried by the global oceans and $25 \%$ of the total heat carried by the ocean and atmosphere together at this latitude (Johns et al., 2011; Ganachaud, 2003; Trenberth and Caron, 2001).

For this reason, the AMOC is a primary cause of interhemispheric asym- 
metries in surface temperatures, precipitation and top-of-the-atmosphere radiation, not only in the Atlantic sector, but also world-wide; with the atmosphere and ocean being slightly warmer $\left(\sim 2^{\circ} \mathrm{C}\right)$ in the Northern Hemisphere $(\mathbb{N H})$ than in the Southern Hemisphere (SH) (Feulner et al., 2013) and the NH emitting slightly more outgoing longwave radiation (Kang et al., 2015), reflecting the compensation accomplished by the coupled atmosphere-ocean system.

The impacts of this northward cross-equatorial oceanic heat transport extend from setting the mean position of the Intertropical Convergence Zone (ITCZ) north of the equator (implying in southward atmospheric heat transport across the equator) (Marshall et al., 2014; Frierson et al., 2013), to affecting North Atlantic multidecadal variability (Zhang et al., 2019), which further impacts many important climate phenomena, including Atlantic hurricane activity, U.S. drought frequency, European temperature/precipitation as well as Sahel/Indian monsoon and northeast Brazil rainfall (Sutton and Hodson, 2005; Ting et al., 2011; Folland et al., 2001; Knight et al., 2006 and references in Zhang et al., 2019).

Moreover, it has been long believed that the release of heat to the atmosphere associated with the oceanic heat transport in the North Atlantic, through Gulf Stream (GS) and North Atlantic Current (NAC) dynamics, contributes to the relatively mild climate in northwest Europe (up to $6^{\circ} \mathrm{C}$ warmer when compared to the regions along the same latitude in North America). However, the relative roles of the ocean and atmosphere in this matter are debated, with the atmosphere believed to play a dominant role on subdecadal timescales and the ocean leading the variability on longer timescales (Palter, 2015; Yamamoto et al., 2015).

Finally, the AMOC also influences Arctic sea-ice extent (Mahajan et al., 2011; Yeager and Robson, 2017), and the oceanic uptake of heat and carbon (Drijfhout et al., 2012; Winton et al., 2013; Kostov et al., 2014; Marshall et al., 2014; Häkkinen et al., 2015; Fontela et al., 2016) - which were previously discussed in more detail on Section 1.2.1. A comprehensive review of the AMOC, focused on its upper cell, is provided by Buckley and Marshall (2016).

The complicated near-surface and deep pathways of the AMOC, as they are known in the present, are illustrated in Figure 5. Of greatest interest here, however, are the numerous upper-ocean trajectories, illustrated in red color. 
Eventually, upwelled deep and bottom waters of the global ocean must return to their source regions, in order to meet mass conservation constraints. This required return flow takes place at shallower depths, managing to primarily bring Indian, Pacific and Southern Ocean thermocline waters on a route back to the Atlantic basin, ultimately crossing the equator and reaching the northern North Atlantic (Gordon, 2001).

This northward upper-limb return flow carrying interocean waters along the meridional extension of the Atlantic basin is certainly the dominant feature of the AMOC, which is predominantly associated with most of the climatic impacts punctuated above; it is referred to as the "AMOC upper limb".

The AMOC upper limb takes place in the wind-driven upper $\sim 1000-1500$ $\mathrm{m}$ of the ocean, therefore strongly interacting with major wind-driven current systems such as the subtropical gyres and the ACC, prior to arriving at deep water formation areas (Van Aken, 2007).

Even though the AMOC northward heat and mass transports have been mostly associated with North Atlantic dynamics and the process of deep water formation (e.g., Biastoch et al., 2008), important pieces of the overturning puzzle take place upstream - particularly within the subtropical South Atlantic, where the interocean waters converge to proceed the northward advection and are further incorporated into the subtropical gyre circulation, resulting in flow redistribution and water mass transformations which shape the upper limb properties and strongly influence the fate of remotely formed waters (Garzoli and Matano, 2011).

\subsubsection{The South Atlantic as a critical crossroad for the AMOC}

A mixture of Pacific and Indian Ocean contributions is received at the southern opposing corners of the SAO basin, through the Drake Passage (the cold water route - Rintoul, 1991) and the Agulhas Leakage (the so-called warm water route - Gordon, 1985). These are blended together and incorporated within the SASG circulation to then continue to the northward flowing upper limb of the AMOC.

More specifically, waters from the cold water route at the southwestern corner follow the South Atlantic Current (SAC) path to the east where they meet 


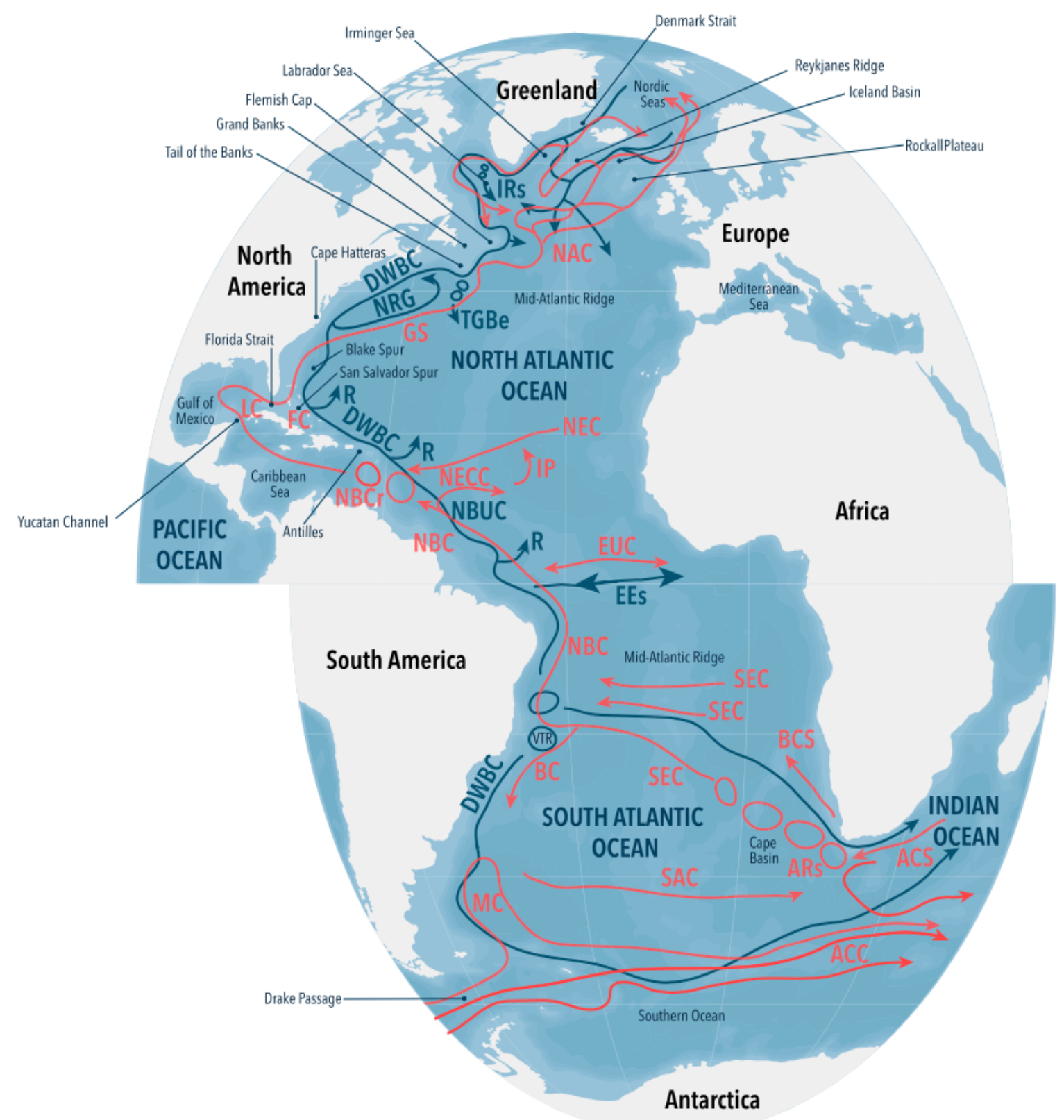

Figure 5 Detailed Atlantic Meridional Overturning Circulation pathways, inferred from Lagrangian observation methods (described in Bower et al. 2019, from where the figure was extracted). Upper (lower) limb pathways are depicted by red (blue). The bathymetry is shaded at 1000-m intervals. The represented ocean currents and features that are relevant for this study are: ACC, Antarctic Circumpolar Current; ACS, Agulhas Current System; ARs, Agulhas Rings; BC, Brazil Current; BCS, Benguela Current System; DWBC, Deep Western Boundary Current; EEs, Equatorial Excursions; EUC, Equatorial Undercurrent; FC, Florida Current; GS, Gulf Stream; IP, interior pathways; MAR, mid-Atlantic Ridge; MC, Malvinas Current; NAC, North Atlantic Current; NBC, North Brazil Current; NBCr, North Brazil Current Rings; NBUC, North Brazil Undercurrent; NEC, North Equatorial Current; NECC, North Equatorial Counter Current; NRG, Northern Recirculation Gyre; R, recirculation; SAC, South Atlantic Current; SEC, South Equatorial Current.

the warm water route at the southern tip of Africa. From thereon, these interocean waters begin their course of northward advection, carried by the Benguela Current 
$(\mathrm{BeC})$ at the eastern boundary of the SASG, which smoothly turns into the southern branch of the South Equatorial Current (SSEC), delimiting the subtropical gyre to the north.

The sSEC heads westward crossing the basin until meeting the South American (SA) coast. At this point, the zonal flow gets divided meridionally between two important large-scale circulation systems. This is accomplished through the origins of two opposing western boundary currents (WBCS): the poleward Brazil Current $(\overrightarrow{B C})$, closing the subtropical gyre circulation, and the equatorward North Brazil Undercurrent (NBUC), continuing to the upper ocean return flow of the MOC (Talley, 2003; Ganachaud, 2003; Lumpkin and Speer, 2003). The sSECNBUC system is also the main conduit for the subtropical-tropical mass exchange (McCreary and Lu, 1994; Malanotte-Rizzoli et al., 2000; Zhang et al., 2003).

The SSEC bifurcation occurs around $15^{\circ} \mathrm{S}$ at the surface (Stramma and England, 1999; Wienders et al., 2000). As it moves southward, the NBUC transport increases and the $\mathrm{BC}$ transport decreases; while as it moves northward, the NBUC transport decreases and the BC transport increases (Rodrigues et al., 2007). Therefore, the SSEC bifurcation latitude (SBL) is indicative of how much subtropical water flows equatorward into the tropical/equatorial region via the NBUC and how much flows poleward, recirculating in the subtropical gyre via the $\mathrm{BC}$. As the origin of the low-latitude western boundary currents, the bifurcation of the SSEC plays significant roles in redistributing water mass and heat meridionally; therefore, it could be indicative of the actual contribution of the westward flowing SSEC to the upper limb of the AMOC.

In sum, the South Atlantic basin provides the gateway by which the AMOC connects with the global ocean. Both in terms of the export of deep waters which are formed within and, mainly, of the return of shallow warm waters to the North Atlantic, which balance the export of NADW. The return path of the AMOC plays an important role in heat and salt transports - critical processes to determine the cause-response relation between AMOC and climate change. 


\subsection{Motivation and Research Questions}

This thesis is motivated, first and generally, by the relevance of the AMOC stability in face of modern, human-induced climate change. Considering that the AMOC has a significant impact on a variety of climate phenomena, with a direct impact on heat transport, sea surface temperature, carbon uptake and nutrients all over the Atlantic basin (Kilbourne et al., 2016), which can result in indirect climate and dynamical impacts worldwide (Buckley and Marshall, 2016).

And, secondly and more specifically, by the role of the South Atlantic Ocean in the global MOC scenario. The SAO is an active player in the transformation of the transiting MOC water masses (Garzoli and Matano, 2011; Garzoli et al., 2013). A significant part of MOC anomalies are originated within the SAO and advected northeastward, making the western boundary current system off Brazil a key region for diagnosing variations in the AMOC and the SASG. Yet, the majority of efforts to understand and measure the MOC variability are focused on the North Atlantic and Southern Oceans, which are the preferential sites for deep-water formation.

The overall motivation of this study is to enhance our understanding of the South Atlantic portion of the AMOC upper limb, assessing the contribution of the boundary currents to the flow and obtaining insights into the dynamics of South Atlantic MOC (SAMOC) variability, in the recent past as well as in the present and near-future climate, considering decadal and longer timescales.

However, in order to address changes in the SAMOC upper limb, we need to address changes in the redistribution of waters along the South American coast after the SSEC bifurcation, and possibly changes in the whole subtropical gyre dynamics as well. It is our hypothesis that variations in the subtropical gyre circulation may reflect variations in the SAMOC upper limb flow and vice versa, once these systems are coupled.

Therefore, Chapter 3 builds upon existing research suggesting recent changes in the circulation of global subtropical gyres with respect to the SAO. Considering that the South Atlantic is a peculiar basin, with strong influence of buoyancy forcing and interocean processes besides the work done by the wind, this study is motivated to find out if and how the SASG circulation is following the 
recurrent global expression in subtropical gyres circulation, in response to changes in atmospheric forcing; and, particularly, how is given the interplay between the wind and buoyancy forcings, as reflected through SAO's basin-wide dynamics.

The motivation behind Chapter 4 is to understand the response of the South Atlantic western boundary current system to pre-industrial climate extremes, during the last millennium - when natural forcing was key to establish climate variability. Considering that the SBL, SASG and AMOC dynamics have been and will continue to be significantly affected by increasing greenhouse gas concentrations in the 21 st century, to better understand what portion of the latest variations might be actually attributable to external forcings and what reflects internal variability, it is crucial to place the modern climate warming in a longer term context.

Lastly, Chapter 5 is motivated and intended to identify and investigate how can SAMOC forced trends possibly evolve over the coming century, amidst uncertainty imposed by the presence of internal climate variability.

Some Research Questions guiding this study are:

1- In a systematic way, how are long-term trends evolving in the South Atlantic large-scale circulation and the upper limb of the AMOC, including its cold and warm water routes?

2- How do CESM1 model simulations represent the South Atlantic and its connections?

3. How is the transmission of low-frequency signals given across the different circulation regimes interacting along the SAMOC upper limb trajectory?

4 How is meridional coherence given across the Atlantic, as seen with a Southern Hemisphere focus and its connection to the North Atlantic Ocean?

5 What is the forced climate change response of South Atlantic circulation pathways, beyond intrinsically generated variability of the climate system?

6 What is the Southern Hemisphere expression of modern AMOC changes? 


\subsection{Objectives}

The general objective of this study consists in investigating the upper South Atlantic MOC variability and its connections with the Southern Ocean and the North Atlantic, in a systematic way, using a set of CESM1 numerical simulations.

The main goal lies in assessing the SAMOC upper limb, monitoring its spatio-temporal evolution and its interplay with the SASG at the western boundary, being the transition point between these two circulation regimes the SBL.

To accomplish this, specific objectives are:

1) to evaluate how the upper limb flow varies in different sub-sections of its downstream extension, mainly in terms of volume transports;

2) to evaluate spatio-temporal variations in the flow structure - such as displacements in dynamical boundary positions;

3) to monitor the basin-integrated MOC streamfunction at different latitudes of the SAO;

4) to analyze spatio-temporal variations in the large-scale wind stress curl and sea surface height fields;

5) to quantify variations in the redistribution of waters between the largescale systems of the SAMOC upper limb versus SASG through the derivation of the SBL index and north/southward transports variation;

6) to simultaneously evaluate how changes in the SAMOC upper limb might relate to changes in SASG circulation dynamics. 


\section{Datasets}

The investigations associated with Chapters 3,4 and 5 are primarily based on simulation results using the CESM ocean component (the CESM1POP2 - Chapter (3) and simulation results from CESM Ensemble Projects - the CESM1(CAM5) Last Millennium Ensemble (Chapter 4) and the CESM1(CAM5) Large Ensemble (Chapter 5).

A set of ocean reanalysis products are also used to perform model validations: the European Center for Medium-Range Weather Forecasts (ECMWF) ocean analysis/reanalysis system 4 (ORAS4), the Geophysical Fluid Dynamics Laboratory (GFDL), the Simple Ocean Data Assimilation version 2.1.6 (SODA216), the Global Ocean Data Assimilation System (GODAS) and the Climate Forecast System Reanalysis (CFSR) from the National Centers for Environmental Prediction (NCEP) (Table 1). The model validations are presented in the supplementary materials listed in Appendix I.

Table 1: Selected time period, horizontal resolution and number of vertical layers above $1000-\mathrm{m}$ of model simulations and ocean reanalysis products.

\begin{tabular}{cccc}
\hline Product & Period & Horiz. Resolution & Vertical Layers \\
\hline CESM1-POP2 & $1970-2015$ & $1^{\circ} \times 1^{\circ}$ & 60 \\
CESM1-LME & $850-1849$ & $1^{\circ} \times 1^{\circ}$ & 60 \\
CESM1-LE & $1920-2100$ & $1^{\circ} \times 1^{\circ}$ & 60 \\
ORAS4 & $1960-2010$ & $1^{\circ} \times 1^{\circ}$ & 26 \\
GFDL & $1961-2010$ & $1^{\circ} \times 0.33^{\circ}-1^{\circ}$ & 34 \\
SODA216 & $1960-2008$ & $0.5^{\circ} \times 0.5^{\circ}$ & 22 \\
GODAS & $1980-2010$ & $1^{\circ} \times 13^{\circ}$ & 31 \\
CFSR & $1980-2010$ & $0.5^{\circ} \times 0.5^{\circ}$ & 31 \\
\hline
\end{tabular}

The CESM is a fully-coupled global climate model which provides stateof-the-art computer simulations of Earth's past, present and future climate states (Hurrell et al., 2013). The coupled components include an atmospheric model (e.g., the Community Atmosphere Model, version 5 - Neale et al., 2010), a land-surface model (e.g., the Community Land Model, version 4 - Lawrence et al., 2009), an ocean model (the Parallel Ocean Program, version 2 - Smith et al., 2010), and a sea ice model (e.g., the Community Ice CodE - Hunke et al., 2015). 
A coupled-climate-system approach is required to meet the challenges of modeling the roles of anthropogenic emissions of carbon dioxide, reactive trace gases and changing land use in the Earth's climate system. The CESM therefore represents the principal components of the climate system and their couplings, aimed at understanding climate variability and global change.

The experiment using the POP2 ocean model is forced with an interannually varying atmospheric data set - the Coordinated Ocean-Ice Reference Experiments phase II (CORE-II). It is here used to investigate simulated windforced changes in the circulation of the SASG. Its spatio-temporally variable advection coefficient (Danabasoglu and Marshall, 2007) allows the model to more realistically capture the circulation response to changing winds (Farneti and Gent, 2011). Further details are provided in Section 3.2 .

The CESM1-LME is used to provide a longer-term perspective for understanding events in the modern record. The 10-member ensemble mean herein used represents the forced variability of the pre-industrial past millennium period, enabling the investigation of climate system responses to natural forcing in a period that precedes significant human-induced changes in Earth's climate. Further details are provided in Section 4.2 .

Finally, the CESM1-LE is employed to obtain a more precise quantification of the time-evolving forced response of ocean circulation changes to anthropogenic global warming, beyond the effect of internal climate variability - from past to future (1920-2100). In the real world, forced climate change signal is superposed by variability that is internally generated by the interacting components of the climate system. This internal variability represents an inherent uncertainty in climate model simulations that is undersampled by multimodel ensembles used in most climate impact studies (Mankin et al., 2020) - which further account for uncertainty arising from differences in model structure. Single-model large ensembles, in turn, are designed to rigorously decompose the forced response from greenhouse gas emissions and the unforced internal variability.

The same model with the same time-evolving external forcing conditions are used to generate several simulations which differ only in terms of their initial condition (small round-off level differences in atmospheric temperature). This is 
enough to ensure that each ensemble member has a unique climate trajectory that represents a possible realization of real world climate change. As they lose their initial condition memory, after a few years to decades (depending on the variable), the trajectories of individual ensemble members randomly diverge due to unpredictable internal variability - causing ensemble spread. Averaging together the CESM-LE 40 ensemble members can effectively cancel out the natural internal variability, resulting in changes only due to forced climate change. Further details are provided in Section 5.2 . 


\section{South Atlantic Subtropical Gyre late twentieth century changes}

\subsection{Introductory remarks}

Anticyclonic subtropical gyres dominate the circulation at midlatitudes in each of the five ocean basins, providing a major pathway for water in the subtropics to be transported to the equator and high latitudes, which is believed to play a key role in modulating the world's climate system (Roemmich et al., 2016). The South Atlantic Ocean (SAO) is characterized by substantial variability at intraseasonal to interdecadal or longer time scales, which has significant impacts on neighboring South America and southern Africa, as well as regions further afield (Wainer and Venegas, 2002). Although the mechanisms associated with this variability remain poorly understood, modulations of the South Atlantic anticyclone and subtropical gyre seem to play a central role (Lübbecke et al., 2014; Cabos et al., 2016).

Over the past century, there has been direct and indirect evidence supporting changes having occurred in the subtropical gyres (Hu et al., 2015). These changes include the intensification (Li et al., 2012, 2013) and poleward expansion (Saenko et al., 2005; Zhang et al., 2013) of these oceanic gyres in both hemispheres, which features a southward shift of the position of zero wind stress curl in the mid-latitudes of the Southern Hemisphere $(\mathrm{SH})$ and a spin-up of the Southern Ocean super-gyre (Alory et al., 2007; Beal et al., 2011; Biastoch et al., 2009; Cai et al., 2005; Cai, 2006; Roemmich et al., 2007; Roemmich, 2007; Saenko et al., 2005) associated with changes in ozone and $\mathrm{CO}_{2}$ forcing (Cai and Cowan, 2007; Arblaster et al., 2011; Polvani et al., 2011) that project onto the Southern Annular Mode (SAM). The SAM is the dominant mode of the SH extratropical circulation (e.g., (Thompson and Solomon, 2002; Marshall, 2003)). Moreover, in conjunction with a systematic change in winds over both hemispheres, an accelerated surface ocean warming has been found along the path of the global subtropical WBCs in association with a synchronous poleward shift of their mid-latitude extensions and/or an intensification in their strength (Wu et al., 2012; Seager and Simpson, 2016; Yang et al., 2016). 
Studies of the long-term variation of the bifurcation of equatorial currents in other ocean basins suggest that there has been a synchronous southward shift of the Pacific North and South Equatorial Current (NEC and SEC) bifurcations over the past 60 years (Chen and Wu (2012) and Zhai et al. (2014), respectively; summarized in Figure 4 from Hu et al. (2015)). These studies focused on individual ocean basins or took a more global perspective. However, little is known about the basin-scale climate variability and long-term changes of the SASG circulation. Except for the numerical coupled model analysis from Wainer et al. (2004), who reported an increased barotropic transport associated with the intensification of the SASG and the ACC under a global warming scenario, and for the recent study by Pontes et al. (2016), which is based on 19 models from the Coupled Model Intercomparison Project phase 5 and projects changes in large-scale features of the SAO circulation under increasing greenhouse gases, previous studies have mainly addressed features located further south, especially the Brazil-Malvinas Confluence (BMC), which is documented as drifting southwards (Goni et al., 2011; Lumpkin and Garzoli, 2011; Combes and Matano, 2014; Pontes et al., 2016).

For the sSEC bifurcation in the SAO, previous studies have only described its mean position (Boebel et al., 1999; Harper, 2000; Malanotte-Rizzoli et al., 2000; Stramma and England, 1999; Wienders et al., 2000; Pereira et al., 2014; Cirano et al., 2006) or even investigated its seasonal variability and the associated mechanisms (Rodrigues et al., 2007, hereinafter referred to as R2007). It is particularly important to better investigate the SAO circulation variability as a whole because it occurs in the only basin that is in direct contact with all the other major oceans and is therefore in a rather unique position to influence their inter-basin exchanges. The pattern of gyre-scale flow in the SAO is strongly influenced by interocean connections, which play an important role in the global Meridional Overturning Circulation (MOC) and permit the thermohaline regimes of neighboring oceans to interact at a variety of timescales (Reid, 1994). By this process, external forcings may induce variability in the South Atlantic boundary currents, which may, in turn, impact the whole of the SASG. Studies of the gyrescale circulation and low-frequency variability in the boundary current regimes may, therefore, provide insights on the variations of the thermohaline circulation 
(Witter and Gordon, 1999).

Based on the aforementioned discussion, the main objective of this work is to answer two principal questions: 1) Is the SASG circulation changing? 2) Does the SBL manifest a long-term trend and, if so, what is the mechanism primarily controlling it?

\subsection{Data and Methods}

This study examined the simulation results from a hindcast experiment with the ocean component of the Community Earth System Model, version 1 (CESM1.0): the Parallel Ocean Program version 2 (POP2), which is hereinafter referred to as CESM-OCN. The CESM ocean component is a level-coordinate ocean general circulation model that solves the three-dimensional primitive equations for ocean dynamics. The version used is based on the POP version 2.1 of the Los Alamos National Laboratory. The ocean model has a $1^{\circ}$ horizontal resolution and 60 vertical levels. It uses a particular parameterization scheme for mesoscale eddy mixing (Gent and Mcwilliams, 1990), which allows non-eddyresolving ocean components of climate models to simulate a degree of eddy compensation (Gent, 2016). The experiment uses the POP2 ocean model forced with the Coordinated Ocean-Ice Reference Experiments interannually varying atmospheric data set (CORE-II; Large and Yeager, 2009) from 1948 to 2015. It is described in Yeager et al. (2018). For the details of the ocean model, its relevant configurations, numerical methods and discretization, the reader is referred to Danabasoglu et al. (2012). Here, we used monthly means of the CESM-OCN data set to investigate changes that have occurred during the period of 1970-2015.

To validate the model results, a set of five ocean reanalysis products was used to compare the mean circulation fields and assess the accuracy in reproducing the SSEC bifurcation: the European Center for Medium-Range Weather Forecasts (ECMWF) ocean analysis/reanalysis system 4 (ORAS4), the Geophysical Fluid Dynamics Laboratory (GFDL), the Simple Ocean Data Assimilation version 2.1.6 (SODA216), the Global Ocean Data Assimilation System (GODAS) and the Climate Forecast System Reanalysis (CFSR) from the National Centers for Environmental Prediction (NCEP) (Table S1). The model validation results 
and statistical analysis procedures are described in the Supporting Information of Marcello et al. (2018) (Yamagami and Tozuka, 2015; Smith, 1997; Mann, 1945; Kendall, 1975; Tokinaga and Xie, 2011; Rodgers and Nicewander, 1988).

\subsubsection{SBL definition}

The SBL index was obtained by first zonally averaging the meridional velocities within a $4^{\circ}$ longitude band off the South American coast and finding, at each time step, the latitude of transition from negative (southward) to positive (northward) velocities, i.e., the zero meridional velocity, in each level of interest of the linearly interpolated vertical profile (at $25 \mathrm{~m}$ intervals). As argued by Chen and $\mathrm{Wu}(2012)$ in the case of the Pacific NBL, the bifurcation latitude defined by meridional velocity is not sensitive to different averaging longitudes. A $4^{\circ}$ longitude band was adopted in order to favor the representation of the WBCs in conjunction with the SSEC bifurcation vertical profile. A $2^{\circ}$ longitude band (as used by R2007) yielded virtually the same results.

\subsubsection{SASG dynamical indices}

Changes in the SASG dynamics were inferred from variations in the wind stress curl (WSC), sea surface height (SSH), barotropic stream function (BSF) and sea surface temperature (SST) fields. Figure — displays their 1970-2015 mean climatology, for reference. The Sverdrup transport (SVT) field was used as an auxiliary tool to consistently capture the relevant changes in the WSC field - once the zonal integration removed small scale zonal variability, while retaining the large-scale signal in which we were interested in. The fields were analyzed in terms of their intensity (magnitude) and spatial fluctuations. More specifically, we aimed to keep track of the temporal evolution 1) of their varying strengths inside the dynamical rims given by the zero contours and 2 ) of the varying position of the gyre boundaries, given by the zero contours. 
(a) Wind stress curl $\left[10^{-8} \mathrm{~N} / \mathrm{m}^{3}\right]$

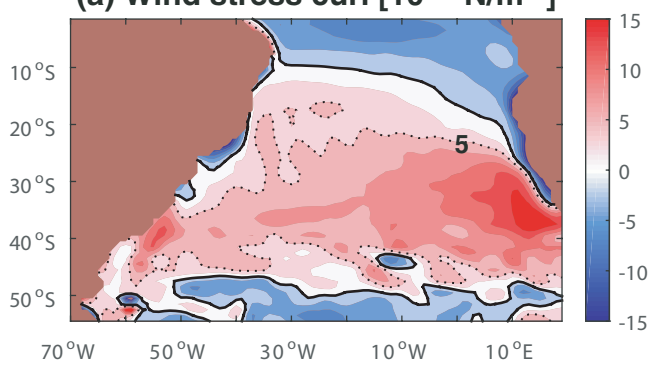

(c) Barotropic stream function [Sv]

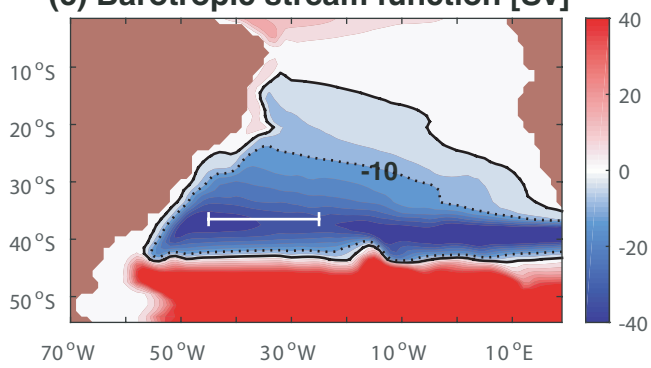

(b) Sea surface height [cm ]

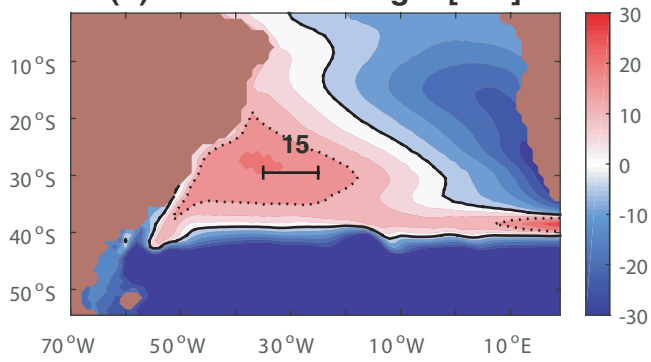

(d) Sea surface temperature $\left[{ }^{\circ} \mathrm{C}\right]$

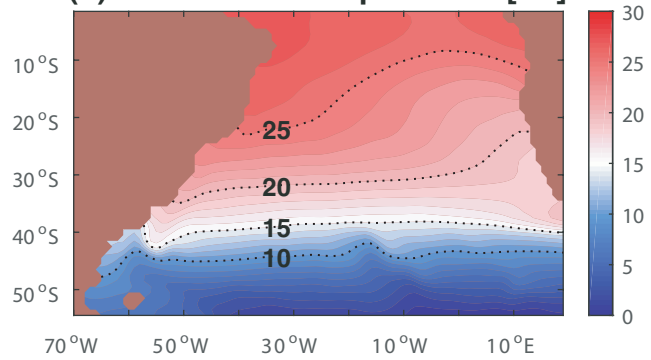

Figure 6 | Large-scale SAO dynamical fields. | 1970-2015 mean climatological (a) WSC, (b) $\mathrm{SSH}$, (c) BSF and (d) SST. Contours are every (a) $2.5 \times 10^{-8} \mathrm{~N} \mathrm{~m}^{-3}$, (b) $5 \mathrm{~cm}$, (c) $5 \mathrm{~Sv}$ and (d) $1^{\circ} \mathrm{C}$. Zero contours that delimitate positive (negative) WSC/SSH (BSF) fields are indicated by black solid lines. Highlighted by black dashed lines are the (a) $5 \mathrm{~N} \mathrm{~m}^{-3}$, (b) $15 \mathrm{~cm}$, (c) $-10 \mathrm{~Sv}$ and (d) $10^{\circ} \mathrm{C}$, $15^{\circ} \mathrm{C}, 20^{\circ} \mathrm{C}, 25^{\circ} \mathrm{C}$ contours; used as parameters to perform index calculations.

\subsection{Results}

\subsubsection{Long-term changes in SASG dynamics}

The basin-averaged differences between 1970-1980 and 2005-2015 (solid versus dashed lines in Figure 7) are more pronounced in the cases of the wind fields (expressed by the changes in TAUX, WSC and SVT) and meridional velocities (VVEL). Nevertheless, relatively modest increasing trends can be seen in all fields. More specifically, in Figure $7 \mathrm{a}$ there is an intensification of the easterly zonal wind stress (TAUX, gray lines) - north of $30^{\circ} \mathrm{S}$, and the westerlies - south of $40^{\circ} \mathrm{S}$. This favors the intensification and poleward displacement of the positive curl (WSC, black lines), especially south of $30^{\circ} \mathrm{S}$. However, despite this overall intensification of the positive WSC south of $20^{\circ} \mathrm{S}$, it seems to weaken slightly between $15^{\circ}-19^{\circ} \mathrm{S}$ (dashed black line in Figure [7a). The variations of the zonal mean SVT naturally reflect those of the WSC, revealing an increased northward basin interior transport implied by the positive WSC intensification, together with the analogous poleward displacement of the system, which is notable mainly south of $45^{\circ} \mathrm{S}$, where the 
dashed lines extend poleward in relation to the solid lines (Figure 7b). Although the zonally averaged SSH ranges approximately from $-125 \mathrm{~cm}$ to $15 \mathrm{~cm}$ across $0^{\circ}-50^{\circ} \mathrm{S}$ in Figure $7 \mathrm{~b}$, the major changes are more confined within the $5^{\circ}-40^{\circ} \mathrm{S}$ meridional extent, with an increase of $6.23 \mathrm{~cm}$ at the $38.5^{\circ} \mathrm{S}$ latitude. In the case of the western portion of the basin, it rises in the constraints of the zero contour, and south of $40^{\circ} \mathrm{S}$, where the sea level field assumes negative values. This also suggests a southward displacement. Accordingly, the variations in the basinwide zonally averaged BSF show increased barotropic transport within the gyre (negative values north of approximately $43^{\circ} \mathrm{S}$ ) with correspondingly decreased barotropic transport south of the zero BSF line, where the SAC and ACC transports occur (Figure $7 \mathrm{~d}$ ). However, this decrease can also be viewed in terms of a general poleward migration. In the case of the surface temperature, the warming seems to be primarily restricted to the region closer to the equator, with major changes confined to the $0^{\circ}-15^{\circ} \mathrm{S}$ extent, mainly, with an increase of $0.81^{\circ} \mathrm{C}$ at about $4^{\circ} \mathrm{S}$ (Figure 7le).

In Figure 7f, the latitude of transition from negative to positive meridional velocities represents the mean SBL for the top $200 \mathrm{~m}$. As northwards (positive) velocities clearly increase, dashed lines (2005-2015) cross the zero VVEL line in more southerly positions than the solid lines (1970-1980). South of $20^{\circ} \mathrm{S}$, the poleward velocities also show a slight increase in magnitude. This could be indicative of an increased flow arriving at the South American coast, which is then distributed between the increased northward and southward boundary currents; the majority of its bulk is directed towards the north, thereby pushing the zonal flow bifurcation latitude to the south.

In the case of the core structure of the gyre, the anomalies of positive (negative) WSC and SSH (BSF) within the zero and the further confined contours of $5 \times 10^{-8} \mathrm{~N} \mathrm{~m}^{-3}$ and $15 \mathrm{~cm} \mathrm{(-10} \mathrm{Sv)} \mathrm{(recall} \mathrm{Figure} 6$ for reference) all show substantial increases, displaying statistically significant positive linear trends after the 80's (Table 2). All the time series mentioned in Table 2 are displayed in Figure S3. They represent the mean value of each field within the indicated contours, in order to highlight how much of each field is really strengthening.

Figure 8 shows the time series "mean intensity per area", which represent 
a) WSC $\left(10^{-7} \mathrm{~N} \cdot \mathrm{m}^{-3}\right)$

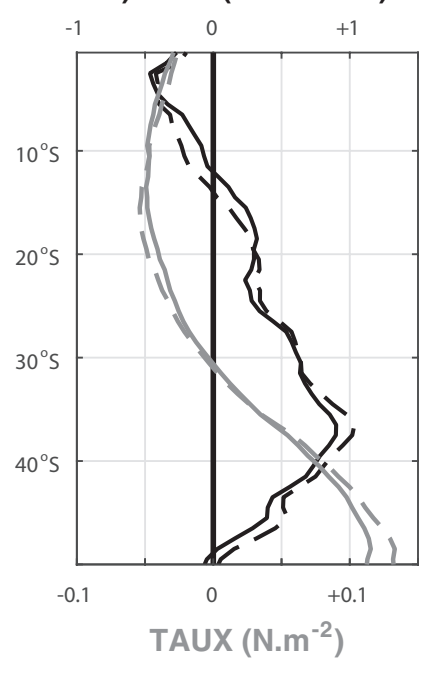

d) BSF (Sv)

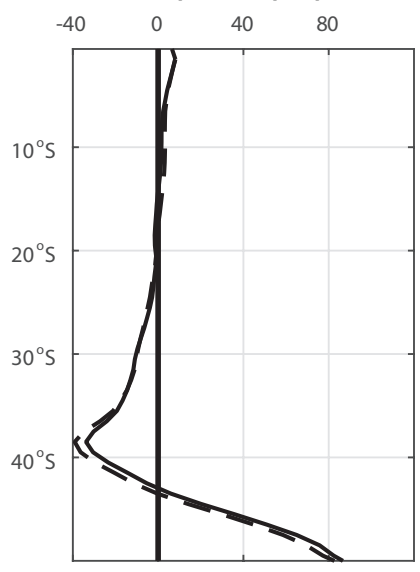

b) SVT (Sv)

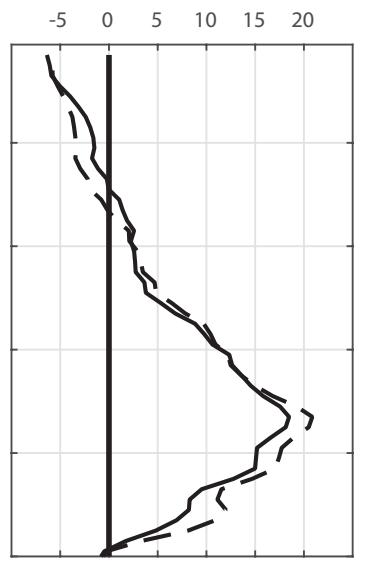

e) SST $\left({ }^{\circ} \mathrm{C}\right)$

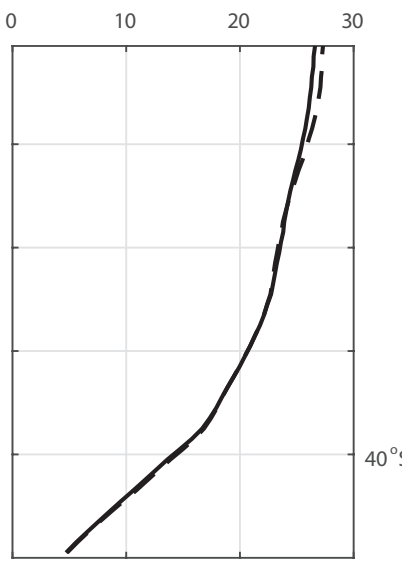

c) $\mathrm{SSH}(\mathrm{cm})$

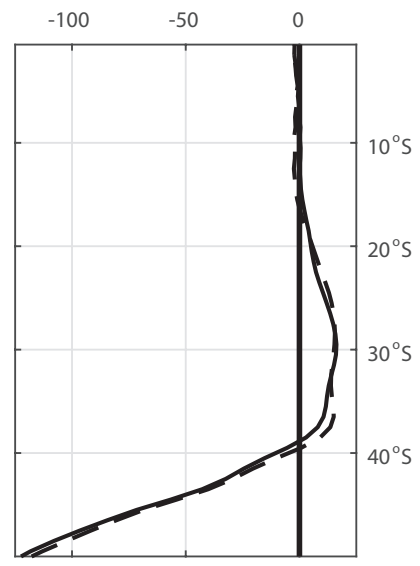

f) VVEL* $\left(m \cdot s^{-1}\right)$

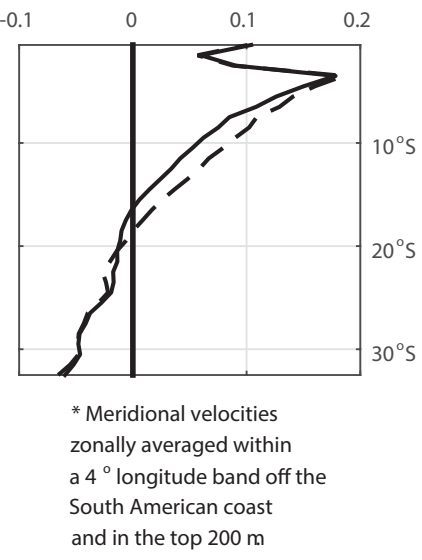

Figure 7 | Transition of South Atlantic zonally averaged fields. | (a) Wind stress curl (black curve) and zonal wind stress (TAUX, gray curve); (b) Sverdrup transport; (c) Sea surface height, from the western boundary up to $10^{\circ} \mathrm{W}$ (recall Figure 1b); (d) Barotropic stream function; (e) Sea surface temperature; and (f) Meridional velocity, from the South American coast (i.e., the western boundary) up to $4^{\circ}$ longitude eastwards. The velocities are also vertically averaged within the top $200 \mathrm{~m}$ layer. Solid (dashed) lines represent the period ranging 1970-1980 (2005-2015). The WSC, TAUX, SVT, BSF and SST are zonally averaged along the entire basin.

the mean value of each field within their zero contour, considering the respective area enclosed by the zero contour at each time step. Still, even considering their filling areas, which might vary as well along time, the conclusion that the WSC, SSH and BSF strengths are indeed increasing does not change. Therefore, Figure 8 expresses the transition from the late 20th into the early 21 st century reasonably well - with increasing values of WSC, SSH and BSF within the SASG domain, 
Table 2: Total period mean value and 1980-2015 increase corresponding to the time series of the mean WSC, SSH and BSF within contours (Figure S3, Marcello et al., 2018). The mean values refer to the corresponding raw time series. The linear trends corresponding to the 1980-2015 increase in a-c, e-f (d) are statistically significant at the $99 \%$ (95\%) confidence level.

\begin{tabular}{lcc}
\hline \multicolumn{1}{c}{ Time series } & Mean value & 1980-2015 increase \\
\hline a) $\mathrm{WSC}>0$ & $7.7810^{-8} \mathrm{~N} \mathrm{~m}^{-3}$ & $1.4510^{-8} \mathrm{~N} \mathrm{~m}^{-3}$ \\
b) $\mathrm{WSC}>510^{-8} \mathrm{~N} \mathrm{~m}^{-3}$ & $10.7110^{-8} \mathrm{~N} \mathrm{~m}^{-3}$ & $1.5610^{-8} \mathrm{~N} \mathrm{~m}^{-3}$ \\
c) $\mathrm{SSH}>0$ & $11.09 \mathrm{~cm}$ & $0.76 \mathrm{~cm}$ \\
d) $\mathrm{SSH}>15 \mathrm{~cm}$ & $18.32 \mathrm{~cm}$ & $0.33 \mathrm{~cm}$ \\
e) $\mathrm{BSF}<0$ & $-15.46 \mathrm{sv}$ & $|-3.33| \mathrm{sv}$ \\
f) $\mathrm{BSF}<-10 \mathrm{sv}$ & $-25.33 \mathrm{sv}$ & $|-3.67| \mathrm{sv}$ \\
\hline
\end{tabular}

obtained from within the respective zero contours that delimitate the governing dynamics of the subtropical gyre circulation (i.e., positive WSC, positive SSH and negative BSF). This result suggests a intensified SASG circulation.

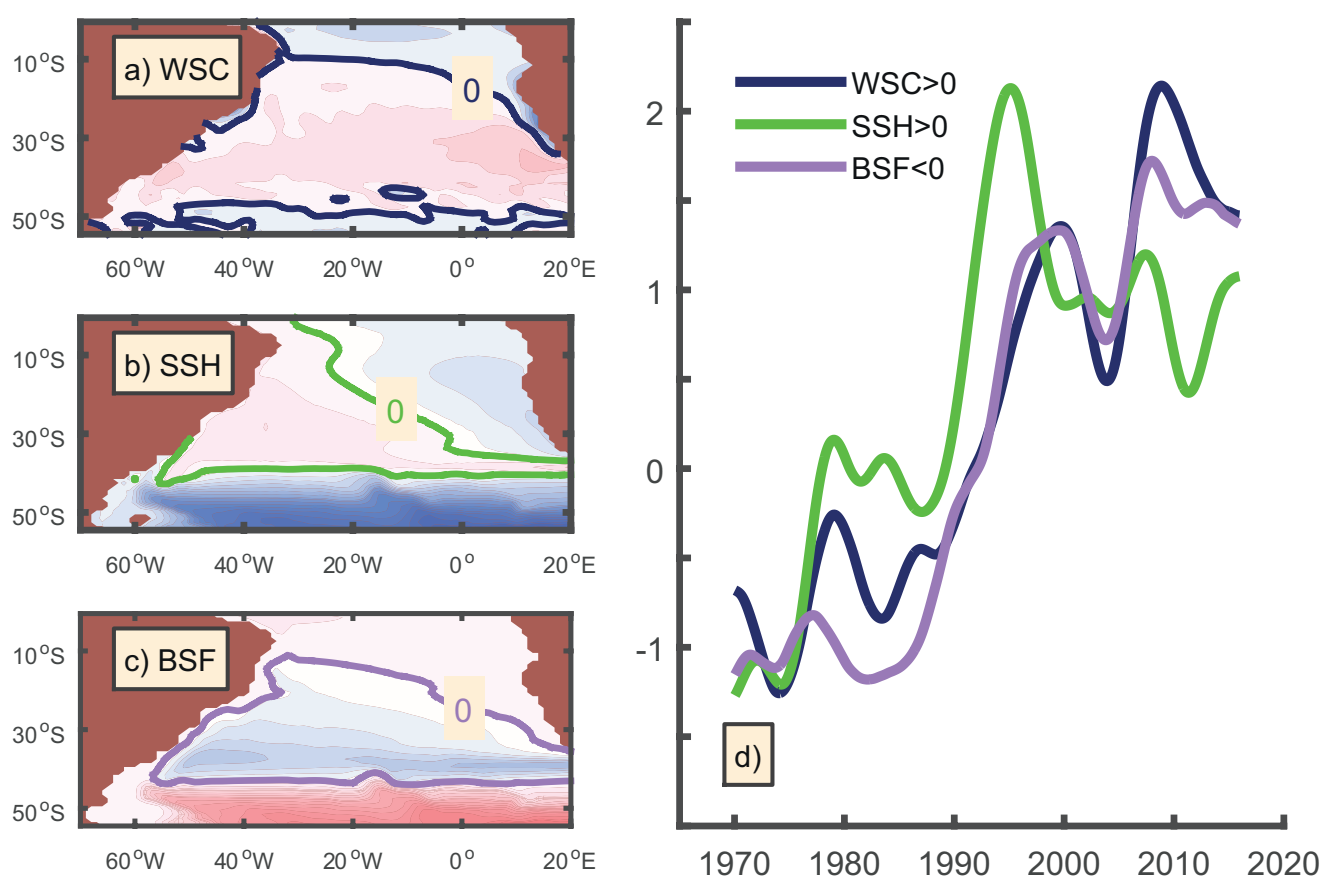

Figure 8 | 21st century intensification of the SASG circulation. | Climatological mean (a) WSC, (b) SSH and (c) BSF fields with their respective zero contours indicated for reference. (d) Low-passed (35-month multi-running mean), normalized (by standard deviation) anomalies of mean WSC (dark blue), SSH (green) and BSF (lilac) per area, within the zero contours.

Time series of the maximum WSC, SSH and $|\mathrm{BSF}|$ and of their respective 
latitudes are displayed in Figure 9. The maximum values show an overall increase, especially after the 80's, while their respective latitudes concurrently migrate southwards. Table 3 details the linear trends in Figure 9. The maximum WSC and BSF increase in magnitude at a similar rate, after the 80 's $(9 \mathrm{a}, \mathrm{e})$. In the case of the maximum SSH, although it presents a statistically significant positive trend, it also has pronounced multidecadal variability, which peaks in 1995 (9k). The latitudes of the maximum WSC and $|\mathrm{BSF}|(9 \mathrm{p}$ and $9 \mathrm{p})$ appear to continuously migrate southward, despite strong high-frequency variability. The latitude of maximum SSH (9d) shifts to the south more abruptly after the 90's, although this southward migration seems to be embedded in increased interannual variability.

a) Maximum WSC

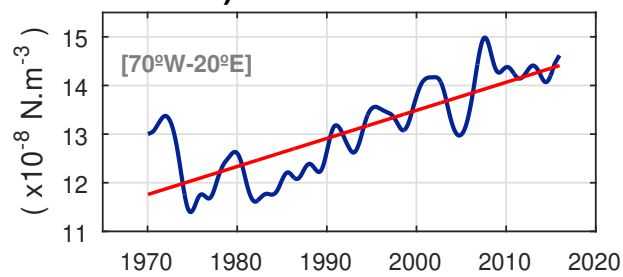

c) Maximum SSH

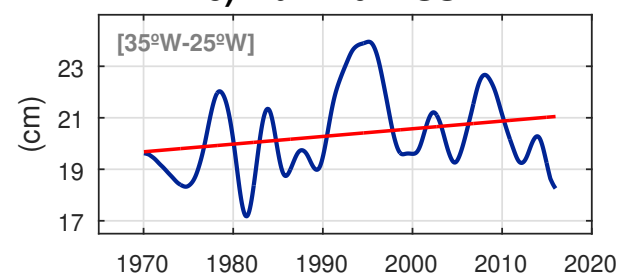

e) Maximum |BSF|

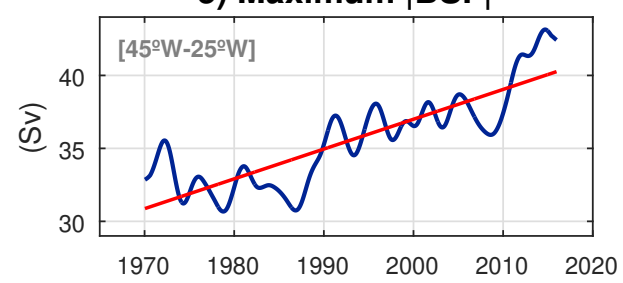

b) Latitude of Max. WSC

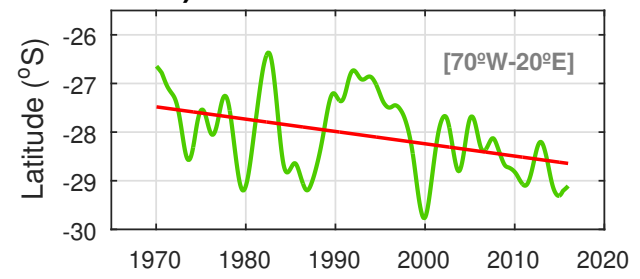

d) Latitude of Max. SSH

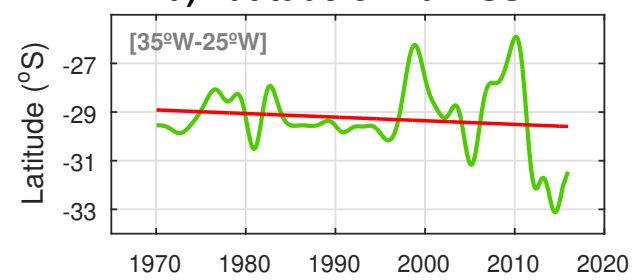

f) Latitude of Max. |BSF|

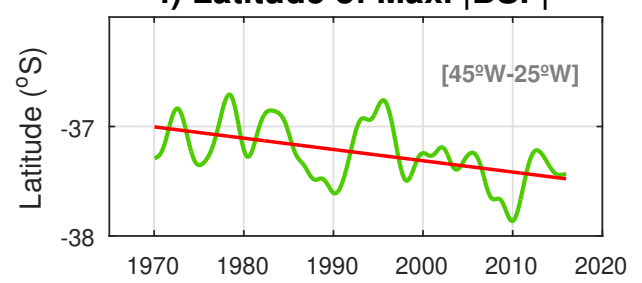

Figure 9 | Intensity and latitude of maximum zonally averaged fields. | Low-passed time series of (a) maximum WSC and (b) respective latitude of maximum WSC averaged over $70^{\circ} \mathrm{W}$ $20^{\circ} \mathrm{E}$; (c) maximum SSH and (d) respective latitude of maximum SSH averaged over $35^{\circ}-25^{\circ} \mathrm{W}$; (e) maximum $|\mathrm{BSF}|$ and (f) respective latitude of maximum $|\mathrm{BSF}|$ averaged over $45^{\circ}-25^{\circ} \mathrm{W}$. The zonal bands used for averaging the SSH and BSF are displayed as black and white labels in Figure $6 \mathrm{~b}, \mathrm{c}$, respectively. The total period linear trends (red lines) in a-c, e-f (d) are statistically significant at the $99 \%(95 \%)$ confidence level.

The robust, recurring change pattern displayed in Figure 10 is the SASG poleward displacement (depicted by the southward shift of isopleths), combined 
Table 3: Linear trends from the time series in Figure 9. The trends corresponding to these particular periods are all statistically significant at the $99 \%$ confidence level.

\begin{tabular}{lcc}
\hline \multicolumn{1}{c}{ Time series } & Period & Linear trend \\
\hline a) Max. WSC & $1980-2015$ & $+2.92 \times 10^{-8} \mathrm{~N} \mathrm{~m}^{-3}$ \\
b) Lat. of Max. WSC & $1970-2015$ & $-1.16^{\circ}$ \\
c) Max. SSH & $1970-2015$ & $+1.37 \mathrm{~cm}$ \\
d) Lat. of Max. SSH & $1975-2015$ & $-1.14^{\circ}$ \\
e) Max. $|\mathrm{BSF}|$ & $1980-2015$ & $+9.39 \mathrm{sv}$ \\
f) Lat. of Max. $|\mathrm{BSF}|$ & $1970-2015$ & $-0.47^{\circ}$ \\
\hline
\end{tabular}
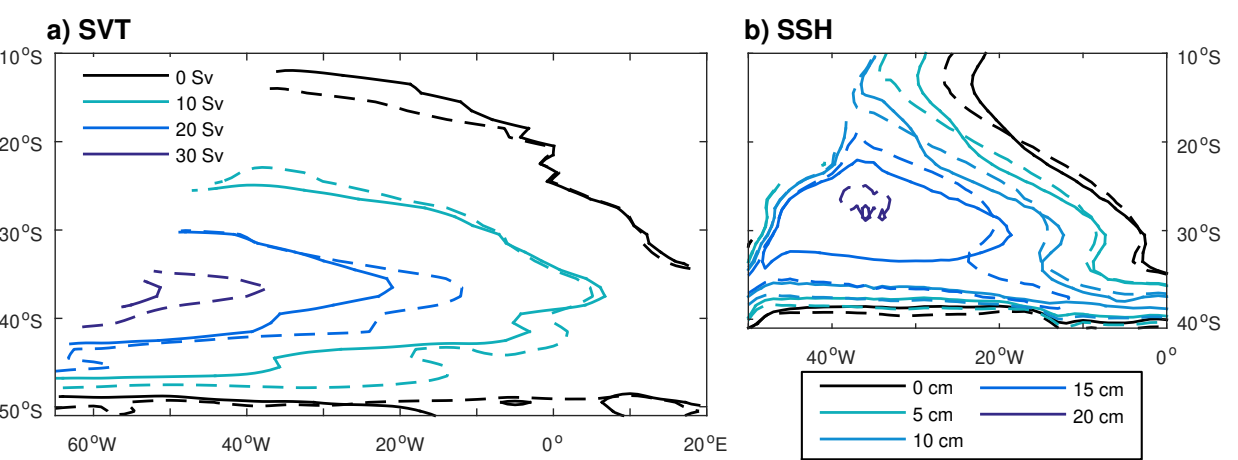

c) BSF

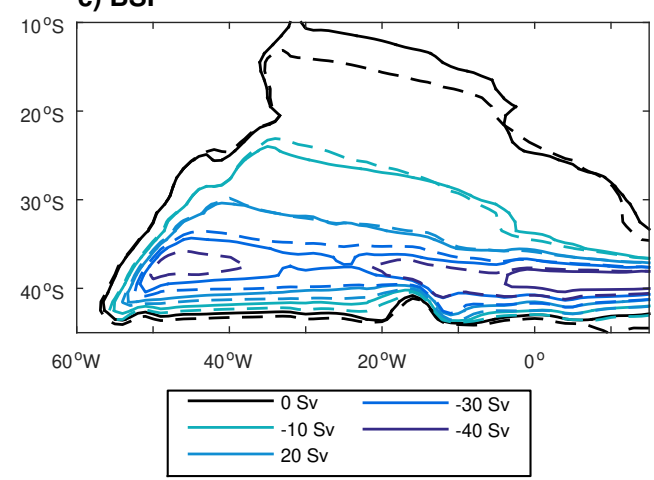

d) SST

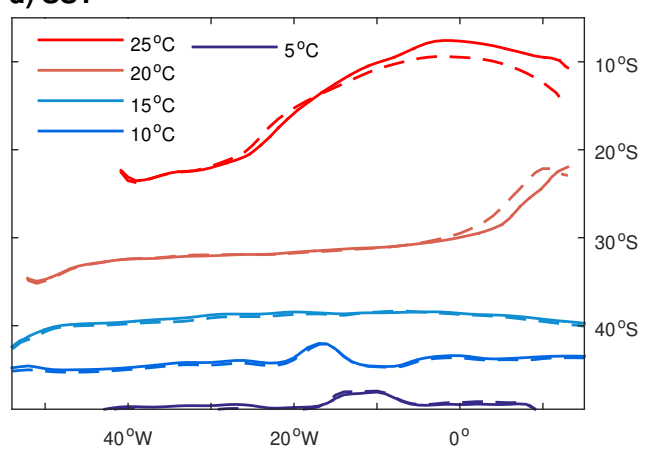

Figure 10 | SASG poleward displacement and core intensification. | Climatological contours for the periods: 1970-1980 (solid lines) and 2005-2015 (dashed lines). Shown are the: (a) SVT, (b) SSH, (c) BSF and (d) SST fields.

with a core intensification, manifested by the enlargement of the regions of higher values in the center of each dynamical field (not considering the SST field). Since the SSH reaches its maximum by the year of 1995 (Figure 8), and it stops increasing from thereon, the difference between the mean 1970-1980 and the mean 
2005-2015 SSH value (Figures [76 and [10b) is less contrasting than for the WSC and BSF (recall their time series in Figure 8, for example).

To better explore the poleward displacement of the SASG system, time series of the dynamical boundaries were derived in regions of high gradients. Defined by the zero contours, they are: the northern edge of the positive SVT field (SVTOn), the southern edge of the positive SSH field in two regions along the basin (SSHOs; $40^{\circ}-20^{\circ} \mathrm{W}$ and $10^{\circ} \mathrm{W}-10^{\circ} \mathrm{E}$ ) and the southern edge of negative BSF field in two regions along the basin (BSF0; $50^{\circ}-20^{\circ} \mathrm{W}$ and $10^{\circ} \mathrm{E}$ ). The spatial pattern of the SST field was monitored by time series of the isotherms of $25^{\circ} \mathrm{C}$ (at $30^{\circ} \mathrm{W}$ ) and $20^{\circ} \mathrm{C}, 15^{\circ} \mathrm{C}$ and $10^{\circ} \mathrm{C}$ (both at $30^{\circ} \mathrm{W}$ and $0^{\circ}$ longitude) (Figure 11).

a) SVTOn

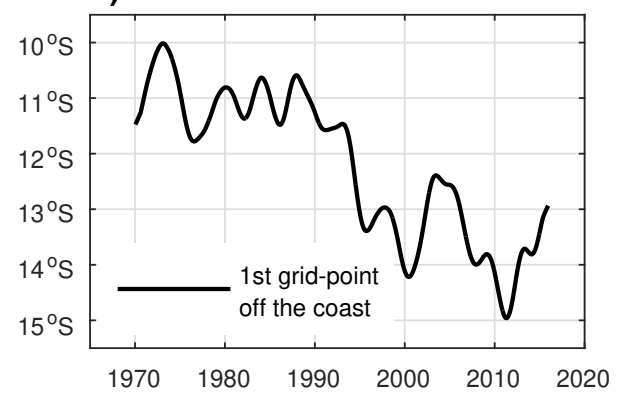

b) SSHOS

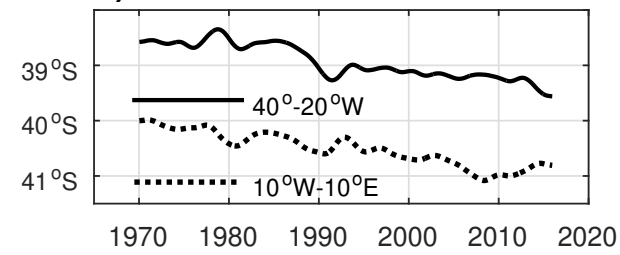

c) BSF0s

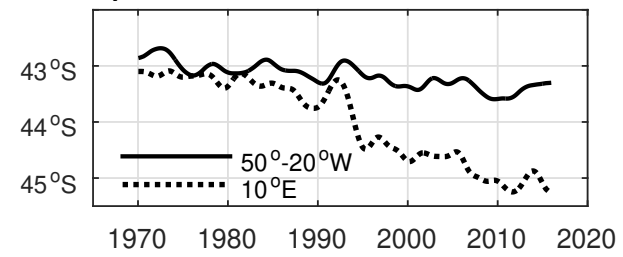

d) SST25-10

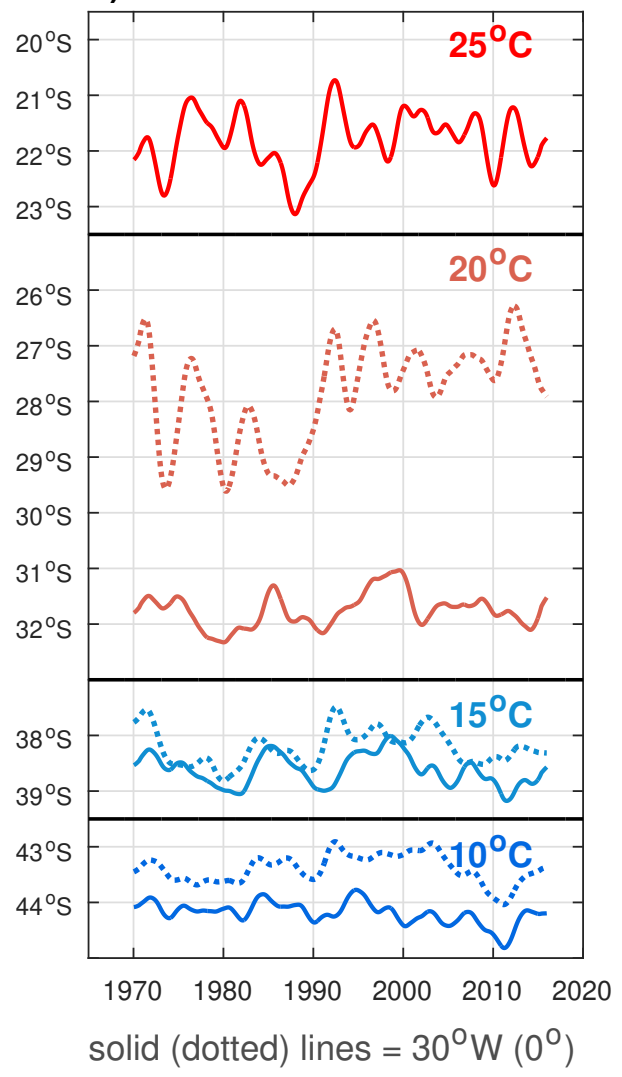

Figure 11 Monitoring the position of SASG dynamical boundaries. | Low-pass-filtered time series of the latitude of zero contour indices derived from regions of high gradient: (a) Latitude of the SVT0 at the northern edge (SVTOn) in the $1^{\text {st }}$ grid point off the South American coast, i.e., the position of zero zonally integrated WSC line; (b) Latitude of the SSHO at the southern edge (SSHOs) in two portions along the basin: a western band ranging $40^{\circ}-20^{\circ} \mathrm{W}$ (solid line) and an eastern one, at $10^{\circ} \mathrm{W}-10^{\circ} \mathrm{E}$ (dashed line); (c) Latitude of the BSF0 at the southern edge (BSF0s) in two portions along the basin as well: a western band ranging $50^{\circ}-20^{\circ} \mathrm{W}$ (solid line) and an eastern section at $10^{\circ} \mathrm{E}$ (dashed line). (d) Latitude of SST contours in two portions along the basin: the $25^{\circ} \mathrm{C}$ isotherm at $30^{\circ} \mathrm{W}$ (red solid line) and the $20^{\circ} \mathrm{C}, 15^{\circ} \mathrm{C}$ and $10^{\circ} \mathrm{C}$ (in light red, light blue and blue solid lines, respectively) isotherms at $30^{\circ} \mathrm{W}$ and $0^{\circ}$ longitude (in solid and dashed lines, respectively). 
The SVTOn, SSHOs and BSFOs indices in Figure 11 all reflect a southward migration. The linear trends are statistically significant at the $99 \%$ confidence level. Table 4 lists the southward shifts corresponding to them.

The SVTOn index, which is the zero zonally integrated wind stress curl line at its upper limit of the SA basin, presents the greater rate of southward migration. This strongly suggests a poleward shift of the northern boundary of the SASG.

In the case of SSHOs, at the southern boundary of the gyre, the contour migrates southwards at the same rate $\left(-0.02^{\circ} \mathrm{S} \mathrm{yr}^{-1}\right)$ at the western $\left(40^{\circ}-20^{\circ} \mathrm{W}\right.$, solid line) and at the eastern $\left(10^{\circ} \mathrm{W}-10^{\circ} \mathrm{E}\right.$, dashed line) portions of the basin. On the other hand, the BSF0s contour interestingly migrates at a much greater rate

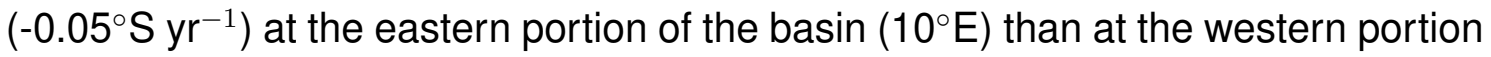
$\left(-0.01^{\circ} \mathrm{S} \mathrm{yr}^{-1}\right.$, along $\left.50^{\circ}-20^{\circ} \mathrm{W}\right)$.

The isotherms do not seem to follow the same variation as the other indices. The $25^{\circ} \mathrm{C}$ isotherm and the $20^{\circ} \mathrm{C}$ isotherm at $0^{\circ}$ longitude appear to undergo a "drifting disruption" before and after the year of 1988, approximately, in addition to pronounced multidecadal variability; only the $10^{\circ} \mathrm{C}$ isotherm, at $30^{\circ} \mathrm{W}$, presents a continuous southward migration, that is statistically significant (Table 4).

Table 4: $1970-2015$ total shift in the time series from Figure 11. All the shifts correspond to statistically significant linear trends, at the $99 \%$ confidence level.

\begin{tabular}{lcc}
\hline Time series & Total shift & Rate $\left(\mathrm{yr}^{-1}\right)$ \\
\hline a) SVTOn & $-4.03^{\circ}$ & $-0.09^{\circ}$ \\
b) SSHOS $\left(40^{\circ}-20^{\circ} \mathrm{W}\right)$ & $-1.00^{\circ}$ & $-0.02^{\circ}$ \\
b) SSH0S $\left(10^{\circ} \mathrm{W}-10^{\circ} \mathrm{E}\right)$ & $-0.99^{\circ}$ & $-0.02^{\circ}$ \\
c) BSF0S $\left(50^{\circ}-20^{\circ} \mathrm{W}\right)$ & $-0.63^{\circ}$ & $-0.01^{\circ}$ \\
c) BSF0S $\left(10^{\circ} \mathrm{E}\right)$ & $-2.49^{\circ}$ & $-0.05^{\circ}$ \\
d) SST10 $10^{\circ} \mathrm{C}\left(30^{\circ} \mathrm{W}\right)$ & $-0.36^{\circ}$ & $-0.01^{\circ}$ \\
\hline
\end{tabular}

\subsubsection{Long-term changes in the SBL}

The long-term evolution of the SBL was explored. To better establish a linkage between changes in the SBL and changes in the surface dynamical fields 
(i.e., WSC, SSH, SST), we focused on the SBL below the surface, at $25 \mathrm{~m}$ depth (hereinafter referred to as SSBL). The smoothed time series shows well-defined low-frequency variability (Figure 12a). The sSBL monthly anomalies are shown in Figure 12k.

An apparent feature depicted in Figures $12 \mathrm{a}, \mathrm{c}$ is the long-term trend of the sSBL. From 1970 to 2015, the mean position of the SSBL has shifted southward from $11^{\circ} \mathrm{S}$ to $16^{\circ} \mathrm{S}$, at a rate of $0.11^{\circ} \pm 0.03^{\circ} \mathrm{S} \mathrm{yr}^{-1}$. The linear trend (orange dashed and solid lines in Figures $12 \mathrm{~b}$ and $12 \mathrm{k}$, respectively) is statistically significant at the $99 \%$ confidence level. Periods between the solid (1970-1980) and dotted (2005-2015) vertical lines in Figure 12a were used to average the $\mathrm{SBL}$ vertical profile in Figure $12 \mathrm{~b}$ - elucidating the poleward displacement at the surface and giving a spatial perspective of the change with increasing depth. It is observed that below approximately $100 \mathrm{~m}$, the scenario reverts to a slight northward migration along the SBL vertical extent, characterizing a decrease in its poleward shift with depth and thus a smaller depth dependence of the subtropical gyre circulation. Figure $12 \mathrm{~d}$ clearly shows the difference between the mean annual cycles of the periods marked in between the solid/dotted vertical lines in Figure $12 \mathrm{a}$. In addition to a southward displacement of the position of each climatological month, a subtle change in their distribution is also observed: the month of the northernmost position switches from November to December.

The rate of the southward migration of the SBL $\left(0.11^{\circ} S \mathrm{yr}^{-1}\right)$ is comparable to that of the SVTOn index $\left(0.09^{\circ} \pm 0.03^{\circ} \mathrm{S} \mathrm{yr}^{-1}\right)$. On longer time scales it is expected that the SBL coincides with the SVTOn, because it is governed by Sverdrup dynamics (Pedlosky, 1996). Here we demonstrate that the long-term change of the low-passed SBL is consistent with the migration of the latitude of the zero WSC line integrated from east to west over the South Atlantic basin.

The linear trend of the SBL at $100 \mathrm{~m}$ also yields a southward migration, of $-0.06^{\circ} \pm 0.02^{\circ} \mathrm{yr}^{-1}$ (Figure S4). It undergoes considerable multidecadal oscillations which are embedded in its overall linear trend, that ends up being way smaller than at the surface (recall Figure 12p). Still, considering the period after the 80 's, the long-term total shift is $-1.94^{\circ}$ (or $-0.05^{\circ} \pm 0.02^{\circ} \mathrm{yr}^{-1}$, statistically significant at the $99 \%$ confidence level). 

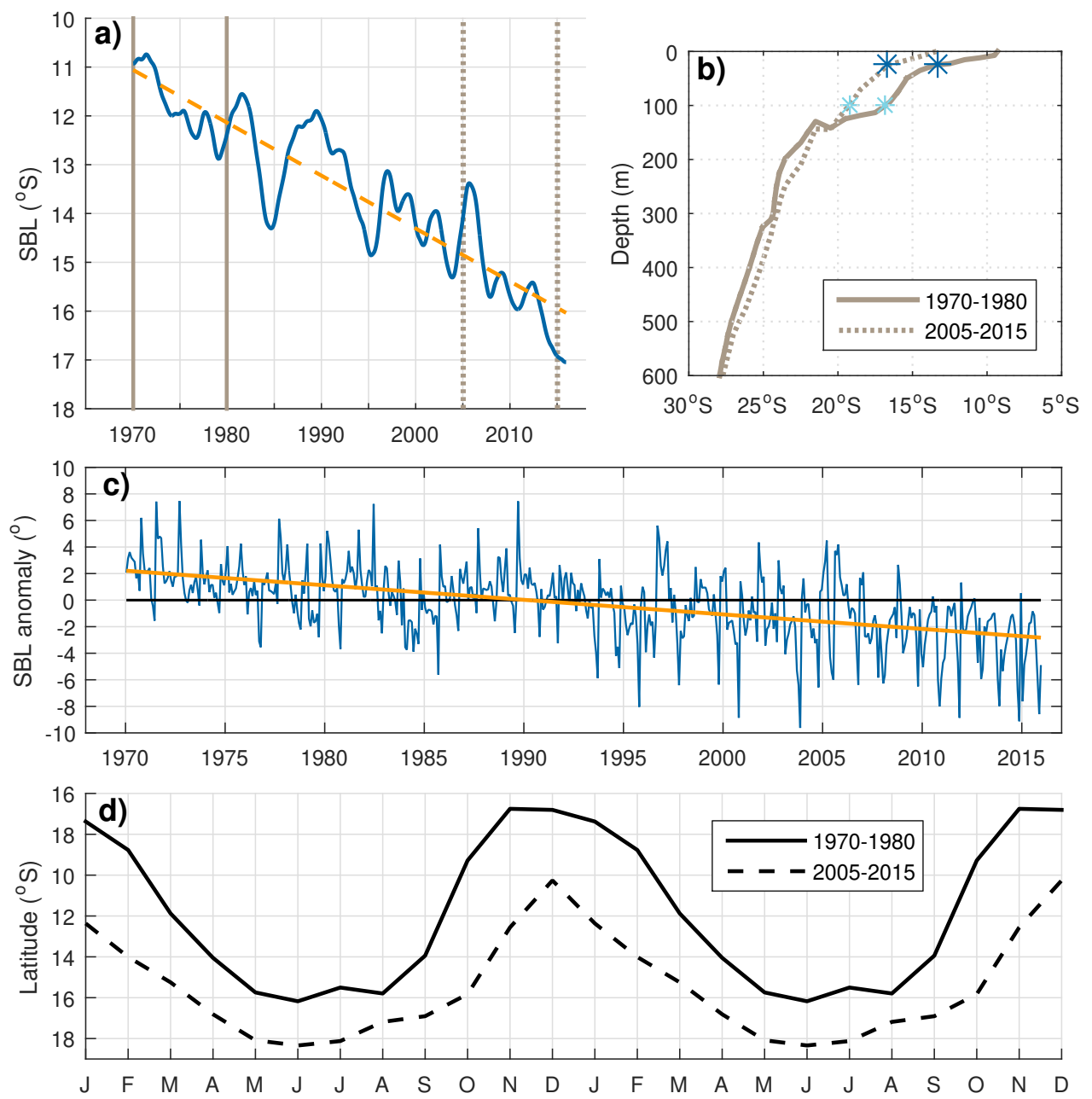

Figure 12 | Long-term change of the SBL. | (a) Low-pass-filtered SBL time series. The orange dashed line indicates the linear trend. Solid (dashed) vertical lines indicate the period of northerly (southerly) SSBL used to average the SSEC bifurcation vertical profile in $12 \mathrm{~b}$ and annual cycle in $12 \mathrm{~d}$. (b) Vertical profile of the SSEC bifurcation (i.e., contour of zero meridional velocity averaged within a $4^{\circ}$ longitude band off the South American coast) averaged during years of northerly SSBL (1970-1980 - solid line) and southerly SSBL (2005-2015 - dashed line), according to the time series in $12 a$. Blue (light blue) asterisks indicate the level used to derive the $S S B L$ time series in $12 a$ (the SBL time series at $100 \mathrm{~m}$, shown in Figure S4). (c) SSBL monthly anomalies (i.e., annual cycle was removed). Orange solid line indicates the linear trend, as in 12a. (d) Climatological SSBL for years of northerly (solid line) and southerly (dashed line) bifurcation position, also according to the time series in $12 \mathrm{a}$. For clarity, the monthly values are plotted for a 2-year period.

Analogous to the discussion of Chen and Wu (2012) about the long-term trend of the Pacific NBL, this type of southward shift in the SBL is associated with a poleward stretching of the boundary between the tropical gyre and the subtropical gyre in the upper western Atlantic Ocean, implying a substantial impact on the origin of the low-latitude WBCs near the South American coast. 


\subsubsection{Long-term changes in SAO current transports}

Following the investigation of changes in the SASG dynamics and the $\mathrm{SBL}$, it now seems suitable to explore changes in the transport of the ocean currents, primarily the SASG northern and western limbs (the SSEC and the BC, respectively) and the low-latitude, equatorward WBC that is formed after the SSEC bifurcation (the NBUC). Thus, the total velocity transports are assessed for the bifurcating SSEC and generated NBUC and BC.

The mean flow field is separated into meridional (Figure $13 \mathrm{a}$ ) and zonal (Figure 13b) velocities. Figure 13 suggests that the South Equatorial Current (SEC) flow diffusely comes across the South Atlantic basin and then spreads around the South American coast, subsequently advancing westwards towards its northern and southern extents (the NBUC and the BC, respectively). On the map of zonal velocities (13b), both of these flows appear as negative velocities, which are progressing upstream to the north or south; however, both are directed to the west, because of the alignment of the coast. Only the initial portion of the NBUC is directed eastward. In the left panel (13a), to the north and south of the mean $\mathrm{SBL}$ (yellow dot), these meridional flows can be more clearly identified as western boundary currents outlining the coast. As a reference, the contour of $|0.02| \mathrm{m} \mathrm{s}^{-1}$ is marked in dashed lines for both the meridional and zonal velocities in Figure 13.

Hereinafter, we ascribe the overall westward flow of the subtropical gyre circulation as $S S E C$, which is the branch known to form the northern limit of the subtropical gyre and to bifurcate into the NBUC and BC. The general westward flow of the SEC includes a part that turns northward near $30^{\circ} \mathrm{W}$ (Stramma, 1991), to form the South Equatorial Countercurrent (SECC), which merges into the complex equatorial current system, eventually giving rise to the northern (nSEC), equatorial (eSEC) and central (CSEC) branches, as well as the South Equatorial Undercurrent (SEUC). This flow configuration can be visualized in detail in Figure 2 from Stramma and England (1999).

Even though Stramma (1991) defines the SSEC as the flow between $10^{\circ} \mathrm{S}$ and $25^{\circ} \mathrm{S}$ and R2007 use the $6^{\circ}-22^{\circ} \mathrm{S}$ band, the use of the different ranges of latitude to derive the westward transport yields negligible differences with respect to the magnitude of the transport; the variability of the time series is not affected. 
Here, we adopt the band of $6^{\circ}-22^{\circ} S$, as in R2007. The sSEC can be understood as a $\mathrm{BeC}$ extension which includes both the $\mathrm{MOC}$ and the return flow of the subtropical gyre (Richardson, 2007).

It is worth mentioning that the northward flow along the Brazil coast between $5^{\circ}$ and $10^{\circ} \mathrm{S}$ occurs as an undercurrent (the NBUC), whereas the nearsurface currents are weak or even southward; only near $5^{\circ} \mathrm{S}$, when it gains an easterly inflow contribution, does the NBUC loses its undercurrent character and is called the NBC (Schott et al., 1998).

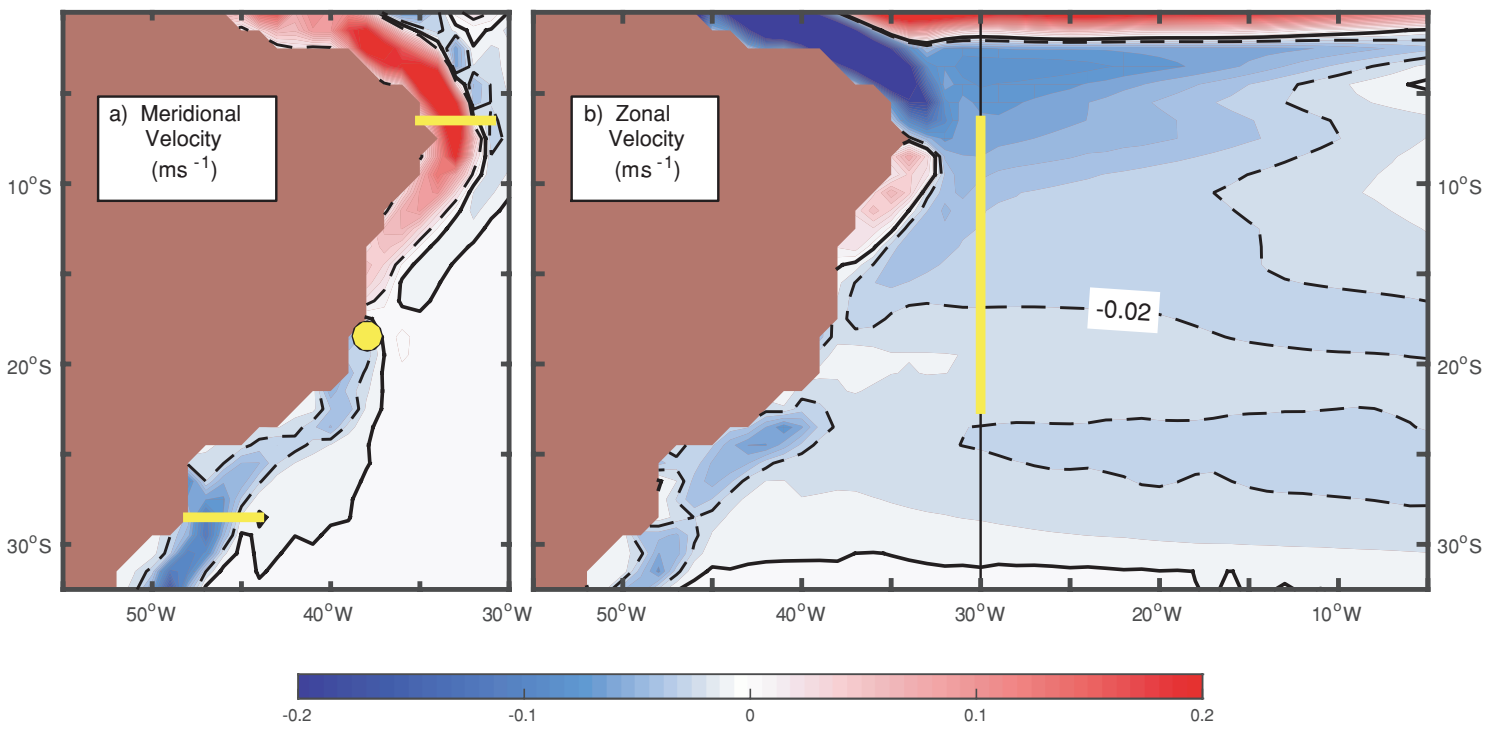

Figure 13 | Climatological flow field (1970-2015). | Depth-integrated horizontal velocities in which positive velocities (northward, eastward) are in red and negative velocities (southward, westward) are in blue. The zero-velocity line is marked in black-bold and the $|0.02| \mathrm{m} \mathrm{s}^{-1}$ contour, used for reference, is marked by the black-dashed lines. The vertical black line along $30^{\circ} \mathrm{W}$ in the right panel (b) indicates where the spatial domain transcends that of the left panel (a). (a) 0-200 m mean meridional velocities. Positive (negative) values denote northward (southward) flow. Yellow dot centered at $19^{\circ} \mathrm{S}$ represents the $0-200 \mathrm{~m}$ mean SBL $\left(<4^{\circ}\right.$ longitude off the coast) and solid yellow lines at $6.5^{\circ} \mathrm{S}$ from $35^{\circ}-32^{\circ} \mathrm{W}$ and at $28.5^{\circ} \mathrm{S}$ from $48^{\circ}-44^{\circ} \mathrm{W}$ represent transects for the calculation of NBUC and BC transports, respectively. (b) 0-200 m mean zonal velocities. Negative (positive) values denote westward (eastward) flow. Solid yellow line at $30^{\circ} \mathrm{W}$ from $6^{\circ}-22^{\circ} \mathrm{S}$ represents the transect for the calculation of the SSEC transport.

As described in Stramma (1991), the SASG has its northernmost current band as the westward flowing SSEC which is found to be a broad sluggish flow, fed by the BeC. Upon approaching the western boundary and splitting into two branches, a small part of the water turns poleward to form the $\mathrm{BC}$, whereas the bulk of the flow contributes to the NBUC. The results presented here confirm this flow pattern, yielding vigorous SSEC and NBUC in contrast with a weak and more steady BC (Figure $14 \mathrm{a}$ and b, respectively; with transect locations indicated in 
Figure 13.

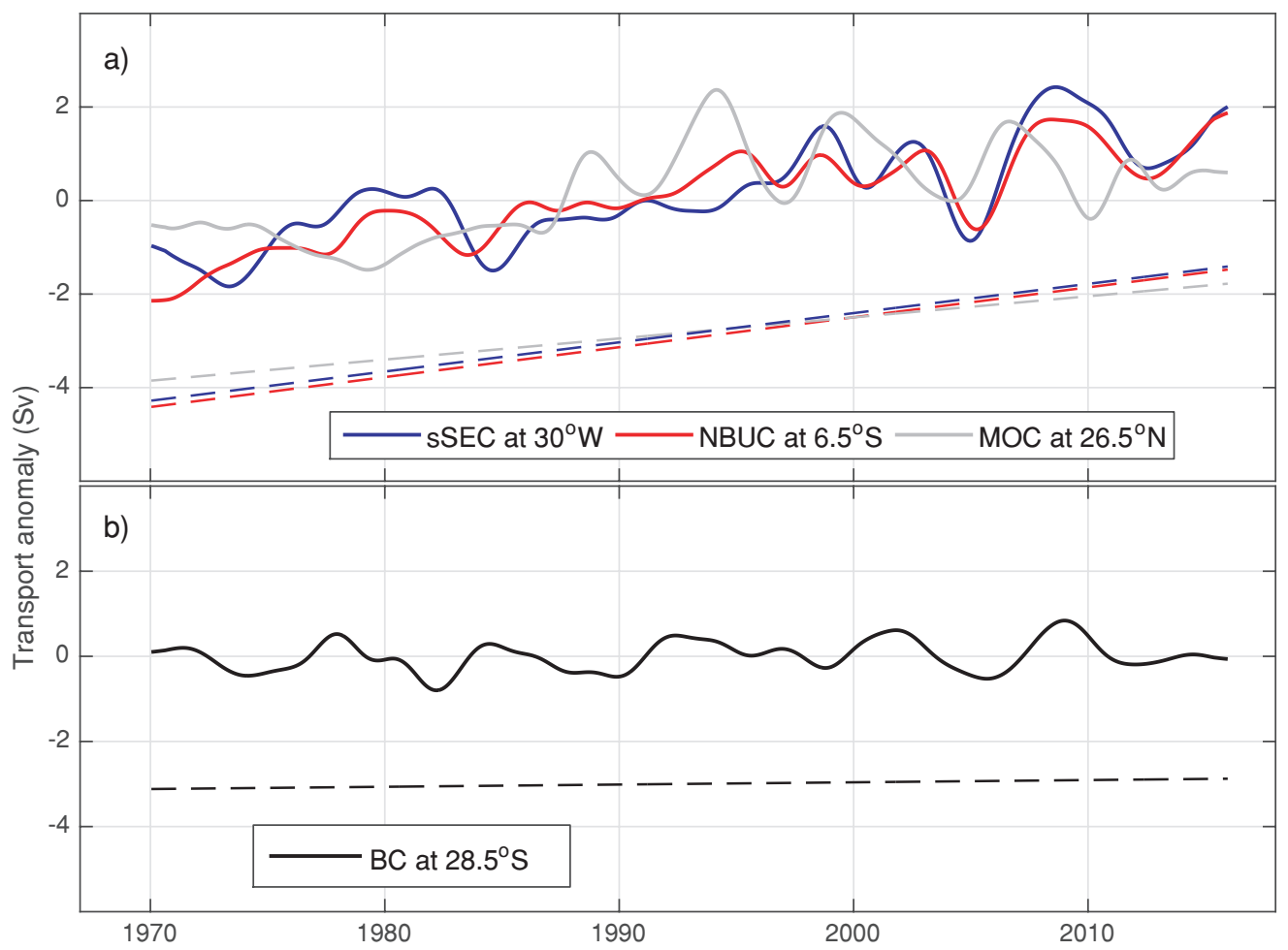

Figure 14 | Boundary current transports and MOC time series. | Low-passed anomalies of the volume transport fluctuations of the (a) westward flowing SSEC at $30^{\circ} \mathrm{W}$, along $6^{\circ}-22^{\circ} \mathrm{S}$ and above $200 \mathrm{~m}$ (blue), about its mean volume of $13.11 \mathrm{~Sv}$; northward flowing NBUC at $6.5^{\circ} \mathrm{S}$, along $35^{\circ}-32^{\circ} \mathrm{W}$ and above $200 \mathrm{~m}$ (red), about its mean volume of $12.53 \mathrm{~Sv}$ and the MOC streamfunction at $26.5^{\circ} \mathrm{N}$, vertically integrated up to the last depth with northward velocities in each time step (light gray), about its mean volume of $19.31 \mathrm{~Sv}$; (b) southward flowing $\mathrm{BC}$ at $28.5^{\circ} \mathrm{S}$, along $48^{\circ}-44^{\circ} \mathrm{W}$ and above $200 \mathrm{~m}$ (black), about its mean volume of $4.32 \mathrm{~Sv}$. For all time series, the dotted lines represent their linear trends, which are reduced by $3 \mathrm{~Sv}$, for clarity. All the linear trends are statistically significant at the $99 \%$ confidence level.

The monthly volume transport anomalies of the sSEC and the NBUC present a peak-to-peak variability of approximately 1.42-1.52 Sv; while, the corresponding value for the $B C$ is only $0.72 \mathrm{~Sv}$. The mean transport values were 13.11 Sv and $12.53 \mathrm{~Sv}$ for the SSEC and the NBUC, respectively, while the BC value was only 4.32 Sv. These values are in close agreement with the literature (R2007, Schott et al. (1998); Stramma and Peterson (1990); Evans and Signorini (1985); Stramma et al. (1990), detailed in the Supporting Information of Marcello et al., 2018).

Finally, the long-term change of the volume transports reveal stronger currents from the 70s on: the strength of the SASG northern (SSEC) and western (BC) limbs increases by $2.87 \mathrm{~Sv}$ and $0.25 \mathrm{~Sv}$, respectively. The linear trends 
shown in Figures 14a, b are statistically significant at the $99 \%$ confidence level. This finding is consistent with the results presented in Figure 8, for example, which suggest an intensified gyre circulation. However, the increase in the northward transport of the NBUC, by $2.94 \mathrm{~Sv}$, is of the same magnitude as that of the aforementioned SSEC transport (2.87 Sv). The correlation coefficient between their standardized monthly anomalies is 0.65 (not shown) and 0.64 for the lowpassed time series (shown in Figure 14). This indicates that most of the increase in the SSEC transport is being directed towards the equator and the $\mathrm{NH}$, through the NBUC. Normally, the larger portion of the SSEC forms the NBUC and crosses the equator, while only a weak part of the SSEC turns southward into the BC (Stramma and England, 1999). The results presented here suggest that the increased anomalous flow of the SSEC reaching the Brazilian shelf is mostly feeding the already stronger NBUC transport, compared to the BC.

Several studies have linked variations in the NBUC transport with the AMOC strength, considering it is inserted in the surface return flow destined to feed the North Atlantic heat sink (e.g., Zhang et al. (2011); Chang et al. (2008); Vellinga and Wu (2004)). To investigate this hypothesis, the basin-integrated MOC transport is derived at $26.5^{\circ} \mathrm{N}$, the latitude where mooring arrays are deployed in order to monitor the AMOC (e.g., McCarthy et al. (2015)). Considering the 1970-2015 period, the resulting time series yields an overall increasing northward MOC recirculation (total increase of $2.07 \mathrm{~Sv}$ ); at a rate similar to those of the SSEC and NBUC transports (all ranging 0.05-0.06 Sv $\mathrm{yr}^{-1}$ ), as shown by the dotted lines in Figure 14a.

It should be noted, however, that this positive linear trend in the AMOC time series is not continuous. It is observed a slight decrease from 1970 until before the 80 's, when it then starts to linearly increase. This increasing rate is mediated by multidecadal variability and, when reaching the end of the 20th century, the AMOC strength seems to stop increasing and enter a period of stabilization (2000-2008) which then leads to a decreasing trend towards the year of 2010. Thereafter the AMOC strength seems to follow a recovery up to 2015 . This behavior pattern is generally in agreement to the one observed in the time series derived using data from ocean moorings at $26^{\circ} \mathrm{N}$ by the RAPID-AMOC programme, which describes a 
gradual AMOC decline over the period of 2004-2014 (Bryden et al., 2014; Srokosz and Bryden, 2015) with an extreme decline in 2009-10 (Goddard et al., 2015; McCarthy et al., 2015).

\subsection{Conclusions and Discussion}

The patterns of gyre-scale flow in global subtropical oceans seem to be changing along with their associated atmospheric forcings under anthropogenic global warming. Using the ocean component of the CESM, the results presented here support our hypothesis that the SAO is indeed subject to these changes. More specifically, it has been suggested that midlatitude subtropical gyres, especially those of the Southern Ocean, are concurrently spinning up their circulations and drifting to southward positions, in response to changes in the surface wind stress field (Roemmich et al., 2007; Cai et al., 2005; Cai, 2006; Saenko et al., 2005; Zhang et al., 2013; Li et al., 2013).

For the 1970-2015 period of the CESM-OCN, the WSC, SSH and BSF fields indicate an intensification of the subtropical gyre circulation, as inferred from increasing values of these diagnostic variables within their dynamical rims of the SASG domain (i.e., their zero contours) which confine the governing dynamics of subtropical SAO circulation: positive WSC, positive SSH and negative BSF. A synchronous poleward migration of the system is also indicated by the behavior of these basin-scale dynamical fields. This is demonstrated by significant differences between the mean climatologies straddling the 80's, as well as linear trends in the time series of the latitude of WSC, SSH and BSF core-maxima and nulledges (generally defined by the zonally averaged maxima and zero contours, respectively).

Although it would be expected that the gyre's spin-up and the associated BC transport increase would push the SASG northern limb equatorward, it has been found that the mean position of the SBL at the northwestern boundary of the gyre shifted southward from $11^{\circ} \mathrm{S}$ to $16^{\circ} \mathrm{S}$ at a rate of $0.11^{\circ} \mathrm{S} \mathrm{yr}^{-1}$. This is at least four times greater than the shift of its Pacific Ocean counterparts (studied by Chen and Wu, 2012 and Zhai et al., 2014).

In addition to agreeing with the reported poleward movement of the whole 
system, this southward shift of the SBL is largely attributable to a substantial increase in the northward transport of the NBUC along the path of the upper branch of the AMOC. When deriving the basin-integrated MOC transport for the $26.5^{\circ} \mathrm{N}$ latitude of the RAPID-MOC observing system, a considerable recirculation increase is found that is consistent with the NBUC intensification. This suggests that the SBL southward shift, accompanied by the NBUC strengthening, might be related to variations in the MOC. Modifications of the water masses participating in the return flow within the South Atlantic can potentially lead to alterations of the thermohaline circulation and the associated meridional heat and freshwater fluxes.

Here we have inferred a spun-up gyre circulation associated with increased torque over the basin (through signals in the WSC field by Sverdrup dynamics and through increased barotropic transport and sea level anomalies). We must clarify that the focus of this study is related to the large-scale scenario associated with low-frequency variability; however, there exist anomalous, higher-frequency variations superimposed on the mean circulation and embedded in these concurrent circulation changes; these are out of the scope of this study.

Our results confirm that important changes are taking place in the evolving upper-ocean circulation, with respect to the SAO. The SASG spin-up and poleward shift are likely to have important consequences on the global climate system, as well as regional climates and ecosystems. As mentioned by Bryden et al. (2012), the key issue for the coming years is to understand how the ocean circulation varies at interannual to decadal time scales and to quantify the impacts of variations in ocean circulation on, for example, the climate and biological productivity. This study aimed to investigate the main large-scale features that constitute the SAO subtropical circulation and describe the associated results and spatiotemporally varying signals, thus providing a dynamical framework to identify potential new states of the system in view of ongoing global climate changes.

* The article version of this work is published online as:

Marcello, F., I. Wainer, and R. R. Rodrigues (2018). South Atlantic Subtropical Gyre late twentieth century changes. Journal of Geophysical Research: Oceans 123(8), 5194-5209. 


\section{South Atlantic surface boundary current system during the Last Millennium in the CESM-LME: the Medieval Climate Anomaly and Little Ice Age}

\subsection{Introductory remarks}

The last millennium (픽 spans the most recent past before anthropogenic forcing became significant, representing an ideal opportunity to understand how the climate system varied under natural conditions (Atwood et al., 2016; Barnett et al., 2005). It is marked by two significant climatic events resulting from natural variability: the Medieval Climate Anomaly (MCA) and the Little Ice Age (LIA). The MCA is generally considered to be a period of above-average temperatures for the years from 950-1249, while the LIA is a period of below-average temperatures from 1400-1699. We use here the definitions of Mann et al. (2009) for each 300-year period.

\section{A Note on MCA and LIA Conventions and Origins}

One should keep in mind that "MCA and LIA" are regarded as the outstanding extremes of a simplistic picture of past global-scale climate variability (Jones and Mann, 2004). There is no consensus of globally-/hemispherically-synchronous MCA and LIA periods (Bradley et al., 2003; Mann et al., 2009; Diaz et al., 2011), since specific timing of peak warm/cold intervals varies regionally (PAGES 2k Consortium, 2013). Considering MCA/LIA spatio-temporal heterogeneity, it is recommended that the general use of such terms is complemented with specific calendar dates, as here, we have mentioned to adopt those of Mann et al. (2009).

When addressing possible causes of these major LM surface temperature anomalies, reconstructions show that the MCA and LIA are both distinct with regard to estimated external radiative forcing of the climate (Jones and Mann, 2004; Solomon et al., 2007). Nevertheless, studies have increasingly pointed to a combination of changes in external forcings with an important role played by internal climate variability (Diaz et al., 2011; Fernández-Donado et al., 2013; 
Neukom et al., 2014; Le et al., 2016; Cheung et al., 2017; Coats and Smerdon, 2017; Wang et al., 2017; Ljungqvist et al., 2019).

The MCA pattern is based on a smaller number of predictors compared to the LIA pattern (Mann et al., 2009). Still, there is indeed evidence of medieval warmth (Graham et al., 2011), although it is not geographically uniform when compared to the recent warming (Diaz et al., 2011). Besides relating to weaker volcanic eruptions and higher solar activity (Crowley, 2000), the MCA-warmth is unlikely to have arisen as a response to external forcing alone. Past studies suggest that solar irradiance changes might have been amplified by internal Earth system feedbacks (Diaz et al., 2011; Gray et al., 2010; Meehl et al., 2008, 2009).

The LIA origins are also associated with external forcings including changes in orbital parameters and weaker solar activity, in turn (Kaufman et al., 2009; Bradley et al., 2003), but more directly to consecutive pulses of volcanism (Miller et al., 2012), which also might have ended up driving internal climate feedbacks (Moreno-Chamarro et al., 2017). Proxies and climate modeling efforts estimate that the MCA-LIA difference in global mean surface temperatures was close to $0.24{ }^{\circ} \mathrm{C}$ (Mann et al., 2009).

The histories of external forcings are not known precisely for the LM, as expected, but they must be imperfectly reconstructed from proxy information sources (Jones and Mann, 2004). That said, there are substantial uncertainties in these reconstructed forcing factors, associated with dating, calibration and specific spatial patterns that are provided for the forcings when these are assimilated into global climate models. These need to be accounted for when interpreting any records and/or model-derived simulations in climate change attribution studies (Schmidt et al., 2011; Otto-Bliesner et al., 2015).

For the North Atlantic Ocean, it has been suggested from proxy evidence the occurrence of an enhanced AMOC during the MCA (Trouet et al., 2009), followed by a weakened AMOC along the MCA/LIA transition (Trouet et al., 2012). Furthermore, using oxygen isotopes and $\mathrm{Mg} / \mathrm{Ca}$ in fossil shells of planktonic foraminifera from high-resolution sediment cores, Lund et al. (2006) showed a weaker Gulf Stream transport through the Florida Strait during the LIA. Nevertheless, for the upstream flow of the northward upper limb of the AMOC, there is little 
or no information on its variations during the LM.

In fact, there is little evidence from proxy data of South Atlantic circulation and dynamics, during either the MCA or the LIA. This can be noted, for instance, when observing the spatial distribution of surface temperature data over the SAO in Mann et al. (2009) or Neukom et al. (2011). Furthermore, Graham et al. (2011) discussed available proxy data for the MCA-LIA period and mentioned a range of studies that showed changes relative to the MCA and LIA for several regions, but not the SAO. Therefore, the behavior of the SAO circulation for the MCA-LIA period is largely unknown. To compensate for this, fully-coupled circulation models allow more detailed examinations of the spatial and temporal variations during the LM in comparison to the proxy data (Landrum et al., 2013).

This study describes the western SAO surface boundary current system response to the naturally-forced events of the MCA and LIA. To do so, simulation results from the Community Earth System Model Last Millennium Ensemble (CESM-LME) experiment are examined (Otto-Bliesner et al., 2015). We focus on the north-south distribution of the westward flow towards the SA coast, which is fed by the $\mathrm{BeC}$ at the eastern boundary of the subtropical gyre. As these waters turn westward, they are broadly spread across the basin and bifurcate. The SSEC bifurcation region marks the point from where interocean waters get divided between two important large-scale features: the SASG and the upper limb of the Atlantic MOC.

The divergence of the SSEC and resulting SASG/AMOC split of interocean waters are the primary motivation of this study. The ultimate goal is to evaluate and characterize the South Atlantic western boundary current (WBC) system response to anomalously warm and cold periods of the LM: the MCA and LIA. Given that the WBC system in the SAO encompasses the SASG, as well as the AMOC, better understanding of the north-south distribution of the SSEC flow can give us insights into the dynamics of both of these circulation regimes. Moreover, the climate variability over the LM provides the essential context for assessing future changes (Bradley et al., 2003). 


\subsection{Data and Methods}

The CESM-LME experiment included a set of simulations forced with reconstructions for the transient evolution of solar intensity, volcanic emissions, greenhouse gases, aerosols, land use/land cover, and orbital parameters, both together and individually, for the period of 850-2006. Output from the ensemble members are available through the Earth System Grid (http://www . earthsystemgrid.org) as single-variable time series. This study examined the ensemble-mean of 10 members with the full-set of external forcings to investigate the period of 850-1849. Monthly outputs were used.

The forcings were applied identically across ensemble members. The only difference among these experiments was the application of a random round-off difference in the air temperature field that initialized each experiment, which would eventually result in ensemble spread. For more details, the reader is referred to the overview paper of the Last Millennium Ensemble Project (Otto-Bliesner et al., 2015). The CESM-LME used a 2-degree nominal resolution in the atmosphere and land components and a 1-degree nominal resolution in the ocean and sea ice components. The CESM 1.1 version used in this experiment was fully documented in Kay et al. (2015). Details on the ocean model, which has 60 vertical levels, were described in ?.

Results from the CESM-LME were recently employed in a wide range of climate variability studies (Wainer and Gent, 2019; Figueiredo Prado et al., 2018; Abram et al., 2016; Huang et al., 2018; Stevenson et al., 2016, 2018; Zambri et al., 2017), which extensively tested the model results against other Earth System Models of the CMIP5 (Zhang et al., 2019) and also showed that the fullforcing realizations reproduced major modes of observed internal climate variability Munoz and Dee (2017); Deser et al. (2012); Ault et al. (2013); Landrum et al. (2013). Figure S2 in the Supporting Material file of Marcello et al. (2019) shows a comparison previously made between the CESM-LME, the ocean component of the CESM (the Parallel Ocean Program Version 2), and five ocean reanalysis products for mean SBL values.

Here, LM-mean defines the mean 850-1849 millennia period, and the 300-year MCA-mean and LIA-mean refer to the mean 950-1249 and 1400-1699 
periods, respectively. The MCA-anomalies and LIA-anomalies were defined with respect to the whole LM period.

To explore variations in the wind-driven, upper ocean circulation associated with the SASG and the upper-limb return flow of the AMOC, depth integrated horizontal velocities above $200 \mathrm{~m}$ were used. This layer better reflects the variability of the WSC field and SSTs, also analyzed here. The large-scale WSC is considered a major forcing mechanism of the upper ocean (Cai, 2006), while SSTs strongly affect ocean-atmosphere coupling and therefore respond to the mean upper ocean circulation. Extending our analyses to deeper layers did not change our conclusions significantly.

\subsection{Results}

\subsubsection{Sea Surface Temperature field}

The MCA and LIA SST anomalies are shown in Figure 15a,b, respectively. As indicated by the dashed contours, the LM-mean SST field was characterized by warmer waters at the tropical region, decreasing nearly exponentially towards higher latitudes, as expected. The MCA-mean SST for the region of study was $14.36{ }^{\circ} \mathrm{C}$, while the LIA-mean SST was $14.25^{\circ} \mathrm{C}$, yielding a MCA-LIA SST difference of $\sim 0.11^{\circ} \mathrm{C}$.

The MCA and LIA anomalous SST fields presented in Figure 15 had a similar spatial distribution, which were opposite in sign. However, the MCAwarming reached a greater amplitude (maximum of $+0.17{ }^{\circ} \mathrm{C}$ at $57^{\circ} \mathrm{W}, 44^{\circ} \mathrm{S}$ ) than the LIA-cooling (maximum cooling of $-0.08^{\circ} \mathrm{C}$ at $22^{\circ} \mathrm{W}, 45^{\circ} \mathrm{S}$ ) for this region. The most remarkable differences were concentrated at the southern limits of the domain, where the maximum MCA-warming lied south of the mean-location of the $\mathrm{BMC}$, and the maximum LIA-cooling was located further east, towards the middle of the basin, where there is a region of amplified cooling spreading zonally and northwestwardly.

The spatial pattern of the SST anomalies in Figures $15 \mathrm{a}, \mathrm{b}$ imply that during the MCA, a region of higher SST anomalies occurred starting at the southeastern interior basin, between $10^{\circ} \mathrm{W}$ and $10^{\circ} \mathrm{E}$, crossing the subtropical SAO, and 
extending toward the SA coast within the $10^{\circ}-20^{\circ} \mathrm{S}$ latitudinal band. These hook-like anomalies characterize the BeC-sSEC path, covering the eastern and northern boundaries of the subtropical gyre. For the LIA period, this same hook-like anomalous feature did not evolve past the eastern portion of the basin, indicating that the cooling remained more confined to the subtropical region, instead of extending up to the western boundary and the equatorial region, compared to the MCA anomalous pattern. The $\mathrm{BC}$ region south of $\sim 25^{\circ} \mathrm{S}$ has higher-amplitude anomalies than in the MCA picture (which actually had as its lower-amplitude a fish-like blob of anomalies centered at this region). This points to more anomalies being advected south during the LIA and to the north during the MCA.
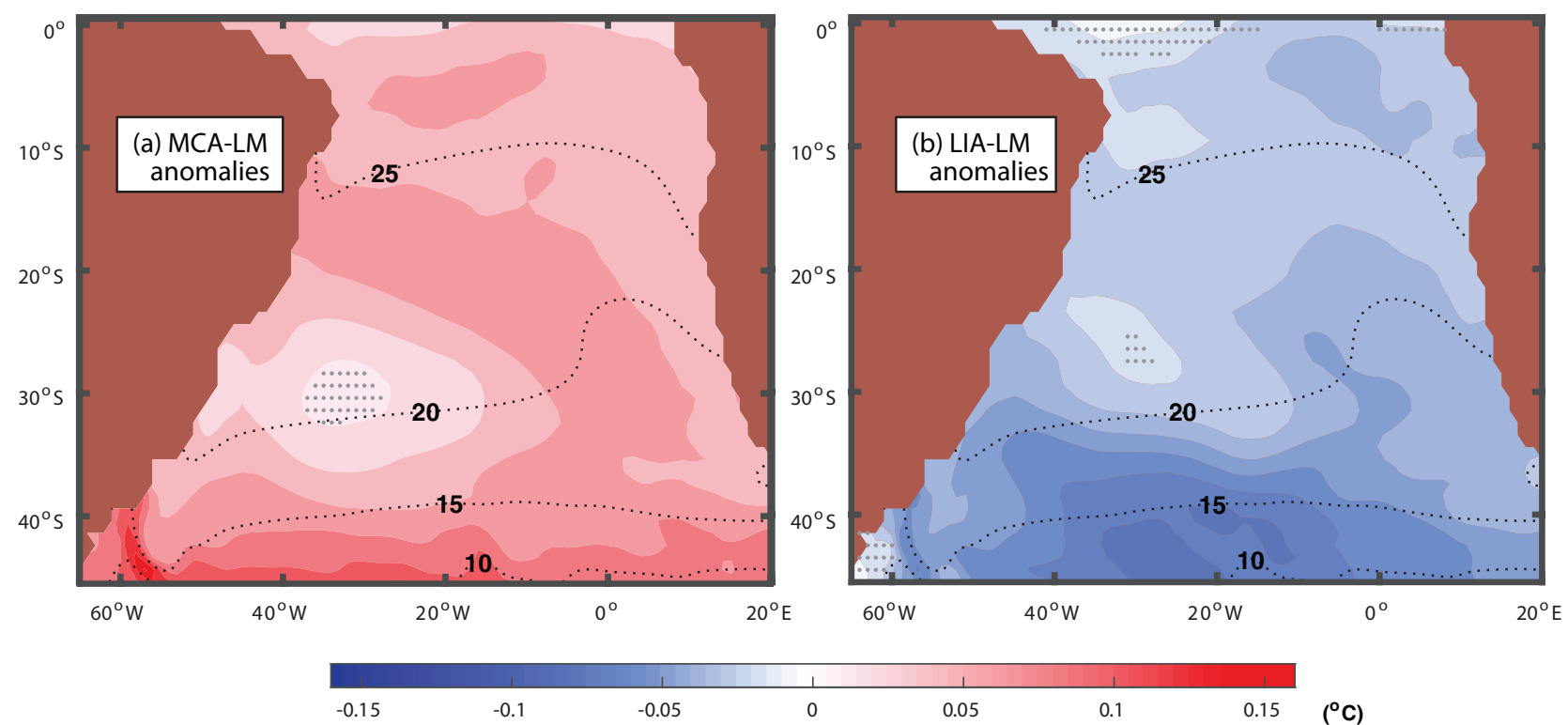

$-0.1$

$-0.05$

0.05

0.1

0.15

( $\left.{ }^{\circ} \mathrm{C}\right)$

Figure 15 Sea surface temperature anomalies $\left({ }^{\circ} \mathrm{C}\right)$ with respect to the whole last millennium (LM) base period (850-1849). (a) For the Medieval Climate Anomaly (MCA), between 950 and 1249, and (b) for the Little Ice Age (LIA), between 1400 and 1699. The black dashed contours represent the LM-mean SST values, for reference. Red (blue) colors represent positive (negative) anomalies. Gray hatched areas indicate non-significant MCA/LIA-LM anomalies according to a two-sided $t$-test $(p<0.05)$.

In fact, anomalous signals lying at the southern boundary of the subtropical gyre tend to follow its anti-cyclonic circulation, being transported northwestward toward the SA coast and then distributed between the northern and southern circulation regimes. The observed MCA/LIA anomalous SST pattern suggests that the course of the northward advection started by the $\mathrm{BeC}$ at the southeastern SAO corner joined primarily the northward AMOC upper limb during the MCA, while during the LIA, it contributed mostly to the SASG, by bending southward after the 
bifurcation.

Our results presented virtually mirrored MCA/LIA SST anomalies, based on their similar spatial distribution, which was opposite in sign. These allowed us to assess the SA circulation system response to past extreme warm/cold climatic conditions, regardless of their distinct causes when compared to modern climate change.

\subsubsection{Horizontal Velocity Field and Volume Transports}

\section{LM-mean circulation field}

The mean SAO upper 200-m circulation field within the region of study was well reproduced by the simulation results of the CESM-LME, according to existing literature (Stramma and England, 1999; Peterson and Stramma, 1991; Stramma, 1991; Stramma and Schott, 1999; Molinari, 1982) (Figure S1).

Background shading in Figure 16 a represents the LM-mean meridional velocity field. To the east of the domain, it depicts the overall southward (blue) and northward (red) interior flows. These mark the division between the complex equatorial current system to the north (blue) and the anticyclonic subtropical circulation to the south (red). To the west of the domain, the narrow path of the opposing WBCs along the South American coast is clear. The superposed vectors indicate the LM-mean horizontal velocities (meridional, as well as zonal velocities). The black contour is the zero meridional velocity line, and the black dot represents the LM-mean SBL at the surface $(25 \mathrm{~m})$, as the point where the meridional velocity averaged within a $4^{\circ}$ longitude band off the SA coast is zero.

The LM-mean zonal velocity for this region (Figure $16 \mathrm{~b}$ ) reveals a predominantly westward flow across the interior basin (blue) towards the western boundary, with the exception of a narrow eastward band close to the equator (red, north of $\sim 3^{\circ}-4^{\circ} \mathrm{S}$ ) which represents the Equatorial Undercurrent (EUC). The zonal upper 200-m currents are identified according to the schematic representation in Figures 16 and 17 from Stramma and England (1999) (Figure S1). Before it crosses the basin toward the SA coast, the SSEC supplies the eastward flow of the South Equatorial Countercurrent (SECC), which partially recirculates in the central branch of the South Equatorial Current (cSEC). The cSEC partially feeds 
the South Equatorial Undercurrent (SEUC) to the east, which heads toward the northern limit of the cyclonic Angola Gyre, further east of the domain (not shown). The cSEC then flows across the basin and provides a contribution to the western boundary. North of that is the southwestern flowing equatorial branch of the South Equatorial Current (eSEC), which branches out of the eastward flowing Equatorial Undercurrent (EUC) at the northern limit of the domain. Eastward and westward velocities along the coastline represent the spread of the WBCs formed north and south of the mean sSEC.

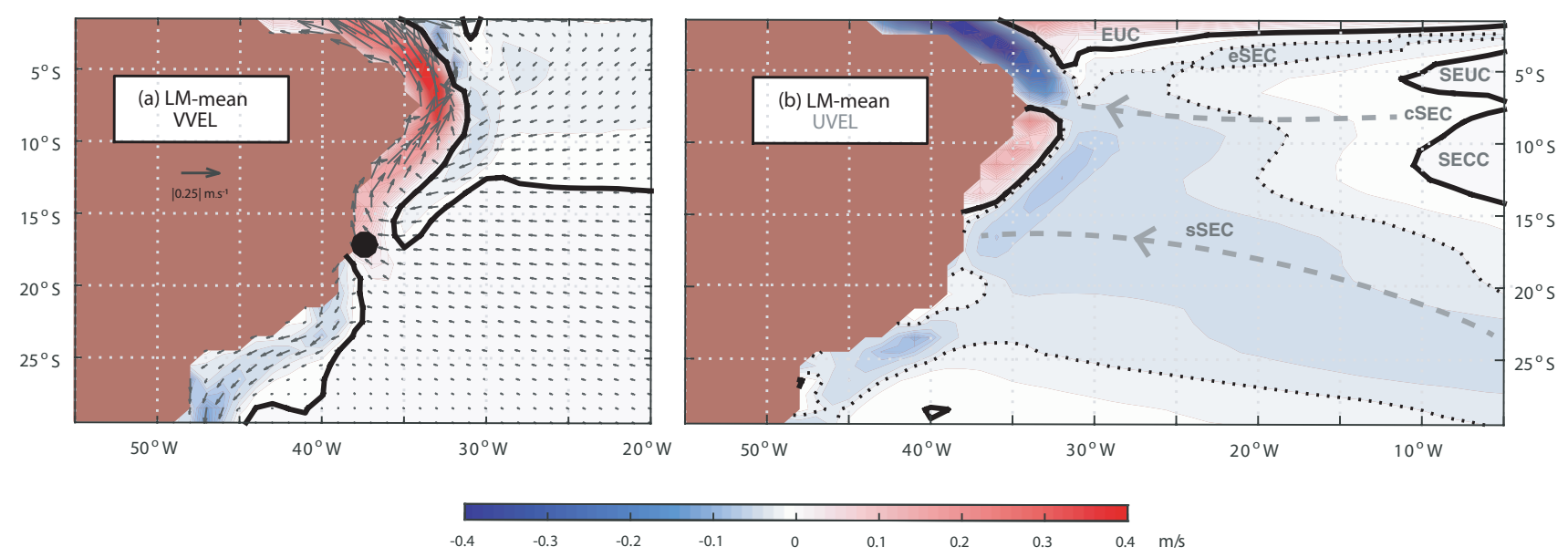

Figure $16 \mathrm{LM}$-mean South Atlantic Ocean (SAO) circulation pattern. Depth-integrated flow over the upper $200 \mathrm{~m}$ for the LM (850-1849): (a) Zonal and meridional velocities (UVEL, VVEL, respectively, as vectors) superposed on the meridional velocities (background colors). Red (blue) denotes positive, northward (negative, southward) velocities. Black contours mark the zero meridional velocity line, and the black dot represents the mean sSEC bifurcation latitude (SBL) at $25 \mathrm{~m}$. (b) Zonal velocities (background colors). Red (blue) denotes positive, eastward (negative, westward) velocities. Black filled contours mark the zero zonal velocity line, and black dotted contours mark the $-0.02 \mathrm{~m} / \mathrm{s}$ contour, for reference. Mean zonal currents from south to north are: the southern branch of the South Equatorial Current (SSEC), the South Equatorial Countercurrent (SECC), the Central branch of the South Equatorial Current (CSEC), the South Equatorial Undercurrent (SEUC), the Equatorial branch of the South Equatorial Current (eSEC), and the Equatorial Undercurrent (EUC). Gray dashed lines indicate the SSEC and CSEC direction, for reference.

The SSEC is the southernmost westward-flowing branch across the SAO that represents the extension of the $\mathrm{BeC}$ and the northern boundary of the SASG Peterson and Stramma (1991); Stramma (1991); Stramma and Schott (1999). It was described by Molinari (1982) as the flow south of the SECC and by Stramma (1991) as the flow between $10^{\circ}-25^{\circ} \mathrm{S}$, east of $30^{\circ} \mathrm{W}$. This is the branch in which we are interested.

Because the Coriolis force acts favoring the western intensification in the subtropical gyre dynamics Stommel (1948), eastern boundary currents such as 
the $\mathrm{BeC}$ are portrayed as broad and diffuse flows. As a consequence, the resulting gyre's shape in the $x-y$ plane can be roughly described by a "D-like" format, rather than circular. Thus, the BeC gradually turns northwestward into the SSEC, by following the counter-clockwise SASG circulation. The SSEC in turn crosses the basin and bifurcates as it approaches the SA coast. The SSEC bifurcation region is the latitudinal band where both WBCs begin to form and diverge. This is nearly between $10^{\circ}$ and $20^{\circ} \mathrm{S}$, around the region of zero meridional velocity (black contour marking the transition between red and blue background interiors in Figure 16a). Part of the SSEC inflow supplies the eastward flow of the SECC east of $30^{\circ} \mathrm{W}$, while most of the SSEC inflow contributes to the NBUC, and only the southern part of the sSEC turns south into the BC Stramma and Schott (1999).

\section{MCA and LIA anomalous circulation field}

To explore the changes in the western SAO circulation field between the MCA and LIA, we analyzed each of their anomalous horizontal flow fields relative to the whole LM integration (850-1849). Background colors in Figure 17 display the meridional velocity (VVEL, (17a, c)) and zonal velocity (UVEL, [17b, d)) anomalies for the MCA (17a, b) and LIA (17k, d). As in Figure 16a, the superposed vectors over the meridional velocities in Figure $17 \mathrm{a}(17 \mathrm{c})$ are the MCA (LIA) anomalous horizontal velocities, highlighting the western boundary anomalies.

Results show clear opposing MCA/LIA anomalous circulation patterns: the MCA was characterized by overall positive VVEL anomalies off the western boundary (Figure 17a), indicating an intensification of the northward NBUC and weakening of the southward BC; while the LIA was subject to overall negative anomalous flows along the coast (Figure 17k) pointing to an NBUC weakening and $\mathrm{BC}$ intensification. This means that the inflow carried by the BeC-sSEC system was being directed mostly northward during the MCA and southward during the LIA.

During the MCA, the increase (decrease) in the NBUC (BC) transport caused the SBL to occur slightly in southerly positions (with an MCA-mean SBL of $17.14^{\circ} \mathrm{S}$ at $25 \mathrm{~m}$ (dark dot in Figure 17a)). On the other hand, the opposite is observed in Figure 17k (with a LIA-mean SBL of $17.00^{\circ} \mathrm{S}$ at $25 \mathrm{~m}$ ). 
The difference between the MCA-mean and the LIA-mean SBL position is therefore of $0.14^{\circ}$ latitude. It is a small difference compared to the model resolution $\left(1^{\circ} \times 1^{\circ}\right)$; nevertheless, considering that the low-passed SBL time series for the 850-1849 total period (whose anomaly time series is displayed in Figure 18a) ranges only from $16.9^{\circ} \mathrm{S}-17.2^{\circ} \mathrm{S}$ at $25 \mathrm{~m}$, with a standard deviation of $0.09^{\circ}$, this $0.14^{\circ}$ difference in position between both periods is considerable. The time series in Figures 18a,b provide the LM-perspective, which evidences the occurrence of a southern (northern) SBL during the MCA (LIA) both near the surface, at $25 \mathrm{~m}$, and at $100 \mathrm{~m}$.
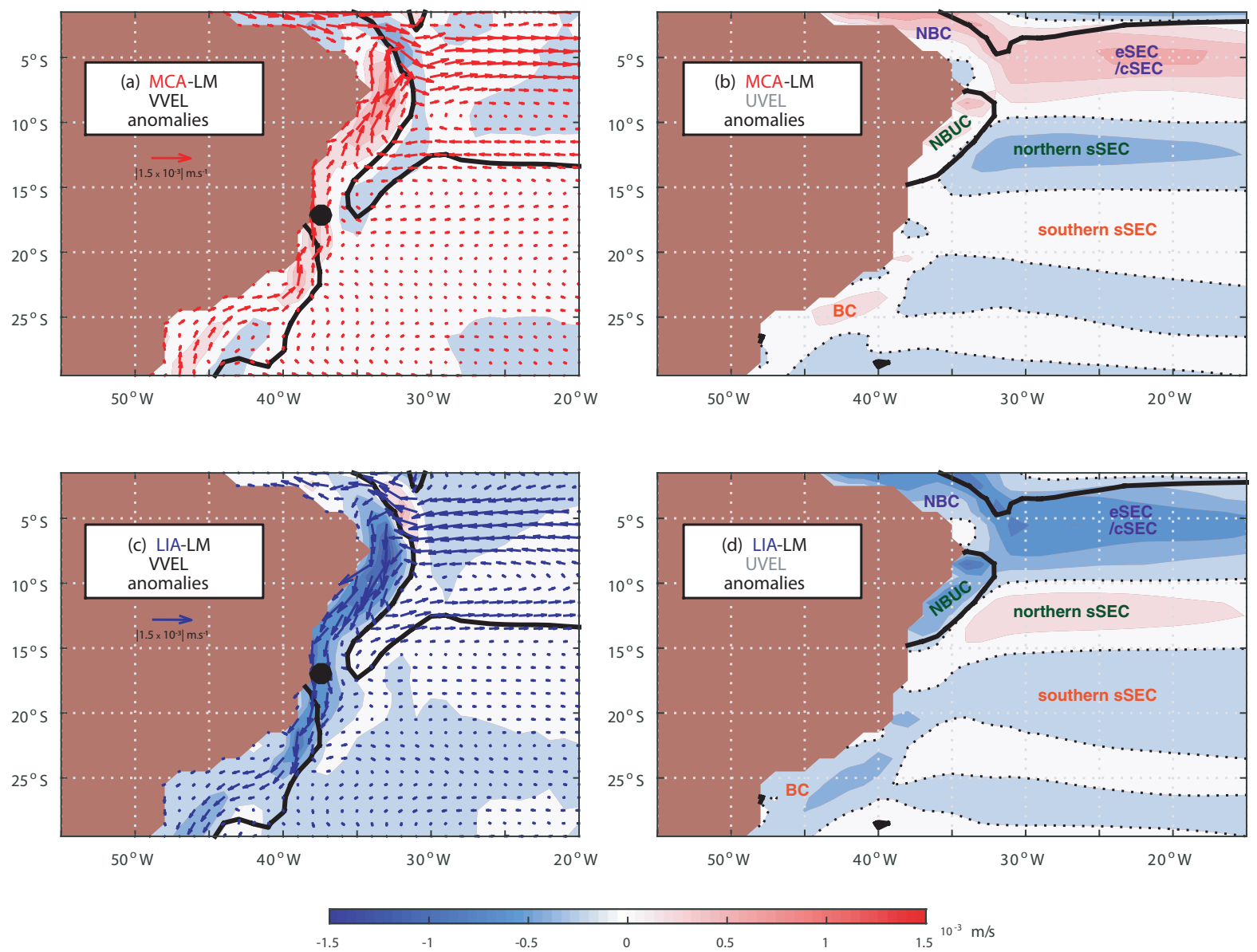

Figure $17 \mathrm{MCA}$ and LIA anomalous SAO circulation pattern. Depth-integrated anomalous flows over the upper $200 \mathrm{~m}$ for the (a,b) MCA (950-1249) and (c,d) LIA (1400-1699) periods. (a,c) Anomalous horizontal velocities (UVEL, VVEL, as vectors) superposed on the anomalous meridional velocities (VVEL, background colors). Red (blue) background colors denote positive, northward (negative, southward) anomalies. Black contours mark the LM-mean zero meridional velocity line, for reference, and the black dot represents the MCA/LIA-mean SBL at $25 \mathrm{~m}$. (b,d) Anomalous zonal velocities (UVEL, background colors). Red (blue) background colors denote positive, eastward (negative, westward) anomalies. Black contours mark the LM-mean zero zonal velocity line, for reference. Indicated are the westward basin-wide currents (eSEC/cSEC, northern and southern SSEC) and western boundary currents (North Brazil Current (NBC), North Brazil Undercurrent (NBUC), and Brazil Current (BC)), identified in different colors according to their respective anomalous signals. 

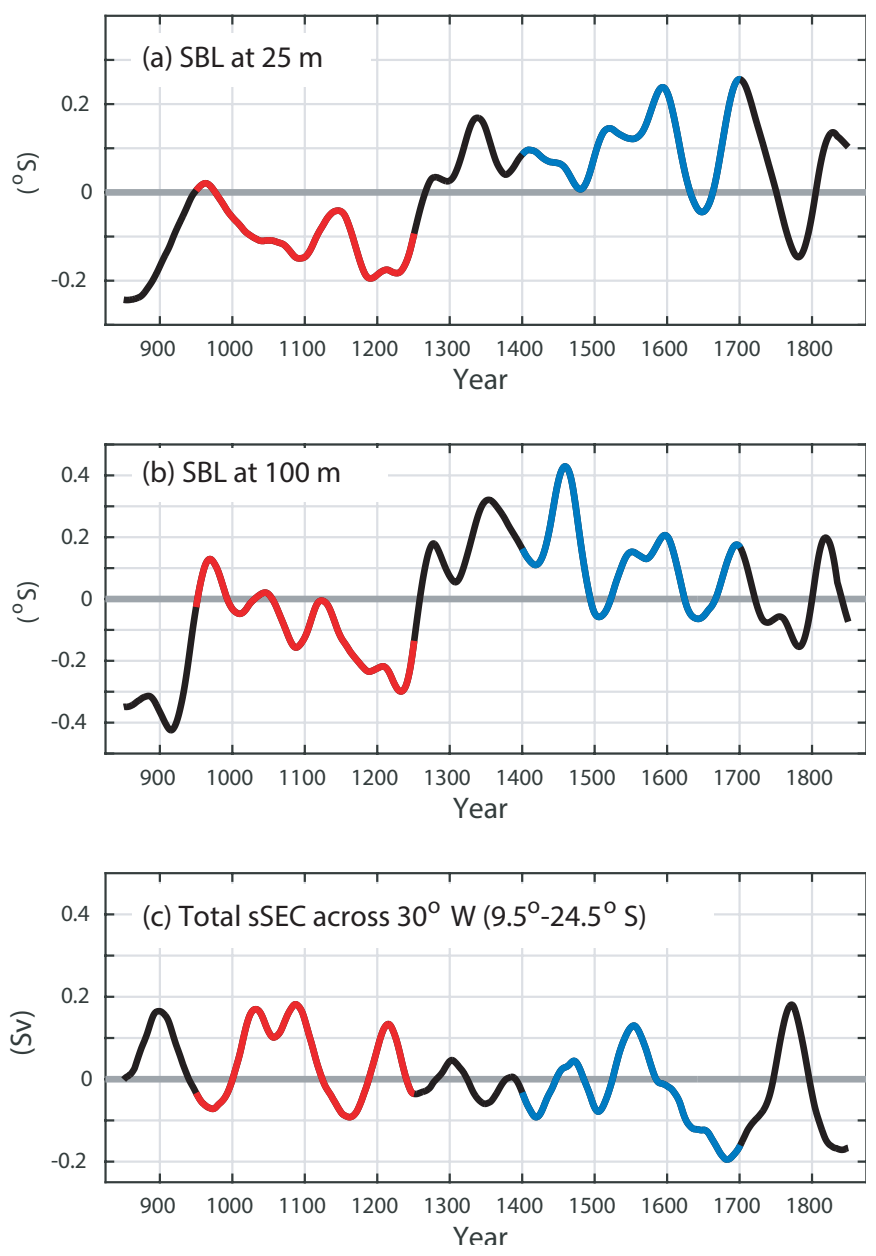

Figure $18 \mathrm{SBL}$ and total sSEC time series. Lowpassed (30-year), standardized anomalies of the SBL at $25 \mathrm{~m}$ (a) and at $100 \mathrm{~m} \mathrm{(b)}$ and of the total westward sSEC transport across $30^{\circ} \mathrm{W}$, between $9.5^{\circ}$ and $24.5^{\circ} \mathrm{S}$ and above 200 $m$ (c). The MCA period is marked in red, while the LIA is marked in blue.

The SST MCA-anomalies shown in Figure 15a reflect this intensification of the AMOC upper limb at this portion: as more warm waters were being transported northward, the BeC-northern SSEC path along the subtropical gyre was marked with greater positive-SST anomalies. In other words, this northwestward, hook-like extension of the positive-SST anomalies agreed with the northward intensification of the flow off the SA coast and southerly SBL position during the MCA as opposed to the LIA.

When looking at the time series of the westward SSEC transport between $9.5^{\circ}$ and $24.5^{\circ} \mathrm{S}$ across $30^{\circ} \mathrm{W}$, according to Stramma (1991) (Figure 18c, herein referred to as "Total SSEC"), one can conclude that it did not change substantially between the MCA (mean 15.97 Sverdrups (Sv), $1 \mathrm{~Sv}=10^{6} \mathrm{~m}^{3} \cdot \mathrm{s}^{-1}$ ) and the LIA (mean $15.92 \mathrm{~Sv}$ ). Only by the end of the LIA period, between 1600 and 1700, the transport anomaly fell further below the zero line (lowering the LIA-mean transport value), returning to a highly positive state right afterwards. Except for that episode, 
the westward transport anomalies fluctuated nearly around the zero line $( \pm 0.06$ Sv standard deviation).

Even though the magnitude of the total westward SEC transport remained roughly the same between both the MCA and LIA periods, the zonal velocities varied differently across latitudinal bands within this $9.5^{\circ}-24.5^{\circ} \mathrm{S}$ band, according to the different SEC branches (Figures 17b,d). In these panels, the westward currents crossing the basin and the WBCs fed by those can be associated according to the banded anomalous flow pattern displayed. These regional patterns of the anomalous zonal velocities elucidated how the westward flow was meridionally distributed throughout the coast between the MCA and LIA, considering that the WBCs' meridional flows straddling the SBL have also a zonal component that follows the coastline orientation. Blue shading denotes negative anomalies, which means an intensification of the mean westward flow, while red shading denotes positive anomalies, which imply a weakening of the mean westward flow at the region.

The zonal band of UVEL anomalies between approximately $16.5^{\circ}$ and $19.5^{\circ} \mathrm{S}$, which we refer to as southern SSEC, seemed to be related to the poleward flowing $\mathrm{BC}$, in which both their anomalies assumed positive values in the MCA, suggesting a weakening of the southern SSEC-BC system, and negative values during the LIA, suggesting in turn an intensification of the system. This can be interpreted as a decrease (increase) of the SASG circulation in its northwestern boundary during the MCA (LIA). Note that the coastline causes the southward BC flow to continue in the westward direction, and this is why both these currents present anomalies of the same sign.

To the north, the zonal band of UVEL anomalies between approximately $9.5^{\circ}$ and $15.5^{\circ} \mathrm{S}$ is defined as the northern SSEC. This portion seems to be contributing directly to the NBUC. The northern sSEC-NBUC system appeared to be anomalously strong during the MCA and anomalously weak during the LIA. It should be noted that the NBUC transport assumes a northeastward orientation at this region due to the coastline. As a result, for the MCA (LIA), the negative (positive) anomalies of the northern SSEC fed into the positive (negative) zonal anomalies of the NBUC. This points to more (less) waters being directed towards 
the Equator and the NH after the sSEC bifurcation during the MCA (LIA).

The regional patterns of the anomalous flow described above suggest an explanation of how the north-south SSEC distribution occurred between the NBUC and the $\mathrm{BC}$ with respect to the warm-MCA and cold-LIA periods. By entering the tropical system, north of $10^{\circ} \mathrm{S}$, the strong band of zonal anomalies observed between approximately $3.5^{\circ}$ and $8.5^{\circ} \mathrm{S}$ (i.e., the anomalous band straddling $5^{\circ} \mathrm{S}$ ) represents the inflow of the cSEC and possibly part of the eSEC to the western boundary. These override the NBUC north of $\sim 5^{\circ} \mathrm{S}$, from where it turns from an undercurrent to a surface intensified current: the North Brazil Current (NBC) Schott et al. (1995). Therefore, we suggest that the cSEC/eSEC anomalies are related to the NBC anomalies observed north of $3.5^{\circ} \mathrm{S}$. They were of opposing sign to the northern sSEC-NBUC anomalies, characterizing a weakened cSEC/eSEC-NBC system during the MCA and an intensified one during the LIA.

Despite the opposing cSEC/eSEC-NBC anomalies with respect to the northern sSEC-NBUC anomalies, what mostly matters for this study is the division of interocean waters carried by the BeC-sSEC between the NBUC and BC circulation regimes. The cSEC actually derives from SSEC recirculation in part (Stramma and Schott, 1996) and then joins the western boundary around $5^{\circ} \mathrm{S}$, transforming the NBUC in the surface-intensified NBC (Schott et al., 1998). This suggests that the high-latitude interocean waters are essentially carried by the SSEC prior to feeding any of its branched systems (i.e., BC, NBUC, cSEC). North of the NBUC, the flow might independently be subject to other external forcings; regardless of that, it has already been sent to the north after the SSEC bifurcation.

We suggest that the band of anomalies south of the southern SSEC, between $20.5^{\circ}$ and $24.5^{\circ} \mathrm{S}$, is contributing both to the $\mathrm{BC}$ and to interior recirculation. The southern SSEC was considered to be between $16.5^{\circ}$ and $19.5^{\circ} \mathrm{S}$ based on the spatial distribution of its MCA/LIA anomalous signals across $30^{\circ} \mathrm{W}$, which correspond to the BC signal, in both the MCA and LIA cases. The distribution of the zonal flow along the coast based on the horizontal anomalies described in Figure 17 was further confirmed by obtaining the volume transport time series. Figure 19 displays the 850-1849 anomalous volume transport time series derived from the horizontal velocity field. The MCA (LIA) period is highlighted in red (blue). 

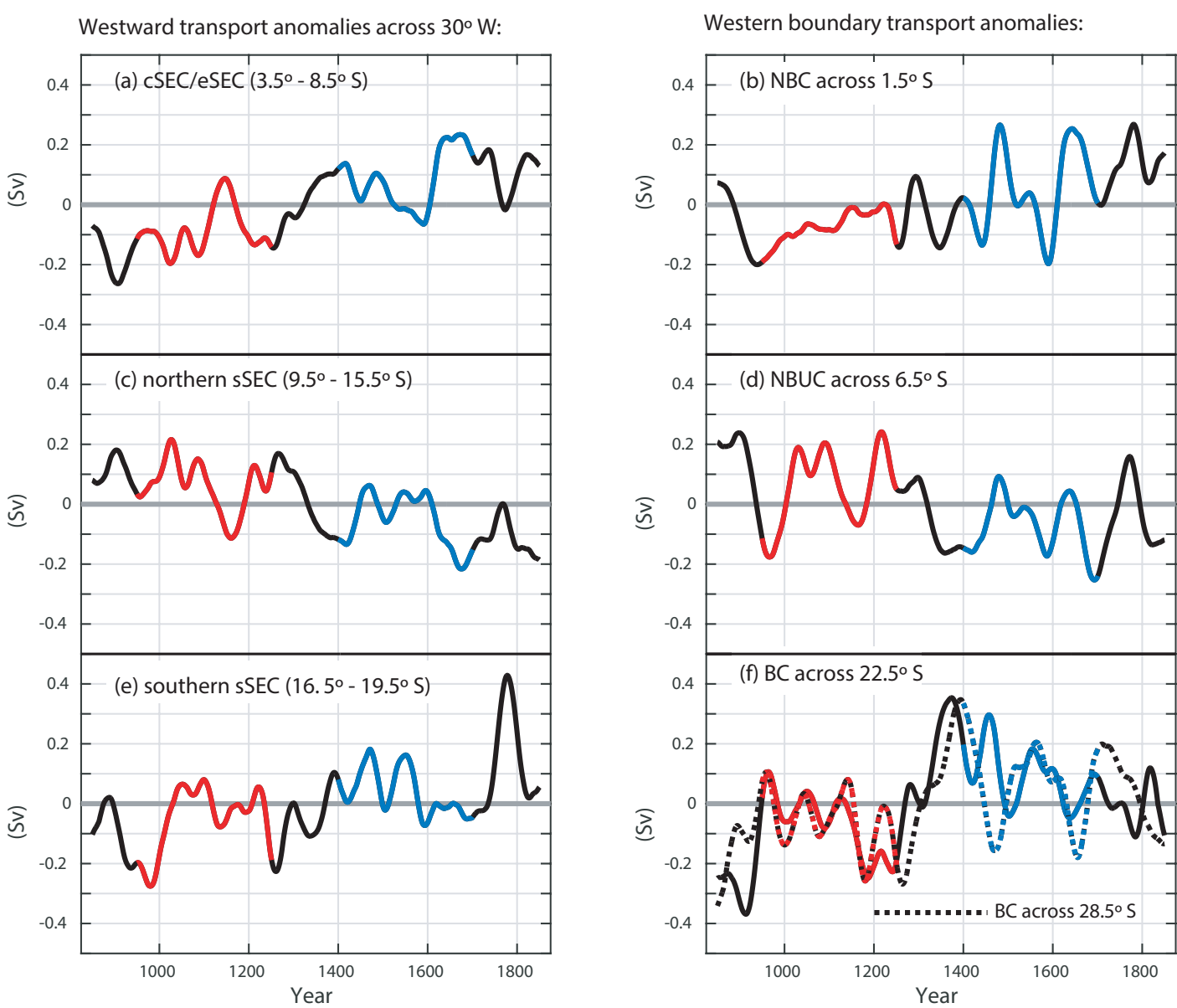

Figure 19 Horizontal volume transport anomalies time series. Low-passed (30-year), standardized anomalies time series of the volume transports above $200 \mathrm{~m}$. (Left column) Westward transports across $30^{\circ} \mathrm{W}$ at different latitudinal bands from north to south: (a) CSEC/eSEC between $3.5^{\circ}$ and $8.5^{\circ} \mathrm{S}$, (c) northern SSEC between $9.5^{\circ}$ and $15.5^{\circ} \mathrm{S}$, and (e) southern SSEC between $16.5^{\circ}$ and $19.5^{\circ} \mathrm{S}$. (Right column) Western boundary transports fed by the westward transports: (b) northward NBC across $1.5^{\circ} \mathrm{S}$, (d) northward NBUC transport across $6.5^{\circ} \mathrm{S}$ (solid lines) and the basin-integrated MOC streamfunction across the same latitude (dashed lines), and (f) southward $\mathrm{BC}$ transport across $22.5^{\circ} \mathrm{S}$ (solid lines) and across $28.5^{\circ} \mathrm{S}$ (dashed lines). The MCA period is marked in red, while the LIA is marked in blue.

The time series from Figures $19 \mathrm{a}-\mathrm{f}$ display an overall similar pattern, especially with respect to their anomalous MCA/LIA signal, supporting our hypothesis that the meridional flow at the western boundary is fed by specific portions of the westward flow coming across the basin. The near-equatorial cSEC/eSEC-NBC system was weaker during the MCA, whilst stronger during the LIA, as well as the southern SSEC-BC system; and the northern SSEC-NBUC system was in turn stronger during the MCA and weaker during the LIA.

Table [5] lists the mean and standard deviation values of the SBL and the volume transports, for the LM, MCA, and LIA periods, corresponding to the time 
series in Figures 18 and 19. Even though MCA-mean and LIA-mean magnitudes seemed quite similar, their associated anomalies had opposite directions in all cases, as shown in Figure 17b,d.

Table 5: LM-mean and standard deviation (std) and MCA/LIA anomalies (MCA-anom., LIA-anom, with respect to the LM-mean) and std (relative to their 300-year intervals) corresponding to the SBL time series in Figure 18a,b and the volume transport time series in Figures 18b and 19a-f. The total sSEC, cSEC/eSEC, northern SSEC, and southern SSEC transports are across $30^{\circ} \mathrm{W}$. Statistically-significant MCA/LIA anomalies are marked in bold, while non-significant ones appear in gray, according to Table 6.

\begin{tabular}{|c|c|c|c|}
\hline Time Series & LM-Mean \pm std & MCA-Anom. \pm std & LIA-Anom. \pm sto \\
\hline $\mathrm{SBL}$ at $25 \mathrm{~m}$ & $17.07^{\circ} \pm 0.09^{\circ} \mathrm{S}$ & $+0.07^{\circ} \pm 0.04^{\circ} S$ & $-0.07^{\circ} \pm 0.05^{\circ} \mathrm{S}$ \\
\hline SBL at $100 \mathrm{~m}$ & $20.46^{\circ} \pm 0.04^{\circ} \mathrm{S}$ & $+0.02^{\circ} \pm 0.02^{\circ} S$ & $-0.03^{\circ} \pm 0.03^{\circ} \mathrm{S}$ \\
\hline total sSEC $\left(9.5^{\circ}-24.5^{\circ} \mathrm{S}\right)$ & $15.94 \pm 0.06 \mathrm{~Sv}$ & $+0.02 \pm 0.05 \mathrm{~Sv}$ & $-0.02 \pm 0.05 \mathrm{~Sv}$ \\
\hline cSEC/eSEC $\left(3.5^{\circ}-8.5^{\circ} \mathrm{S}\right)$ & $6.71 \pm 0.09 \mathrm{~Sv}$ & $-0.06 \pm 0.05 \mathrm{~Sv}$ & $+0.06 \pm 0.07 \mathrm{~Sv}$ \\
\hline NBC across $1.5^{\circ} \mathrm{S}$ & $13.97 \pm 0.05 \mathrm{~Sv}$ & $-0.03 \pm 0.02 \mathrm{~Sv}$ & $+0.03 \pm 0.04 \mathrm{~Sv}$ \\
\hline northern sSEC $\left(9.5^{\circ}-15.5^{\circ} \mathrm{S}\right)$ & $8.76 \pm 0.06 \mathrm{~Sv}$ & $+0.03 \pm 0.04 \mathrm{~Sv}$ & $-0.03 \pm 0.04 \mathrm{~Sv}$ \\
\hline NBUC across $6.5^{\circ} \mathrm{S}$ & $20.48 \pm 0.07 \mathrm{~Sv}$ & $+0.03 \pm 0.06 \mathrm{~Sv}$ & $-0.04 \pm 0.05 \mathrm{~Sv}$ \\
\hline southern sSEC $\left(16.5^{\circ}-19.5^{\circ} \mathrm{S}\right)$ & $3.94 \pm 0.02 \mathrm{~Sv}$ & $-0.01 \pm 0.02 \mathrm{~Sv}$ & $+0.01 \pm 0.01 \mathrm{~Sv}$ \\
\hline $\mathrm{BC}$ across $22.5^{\circ} \mathrm{S}$ & $2.88 \pm 0.04 \mathrm{~Sv}$ & $-0.02 \pm 0.02 \mathrm{~Sv}$ & $+0.02 \pm 0.02 \mathrm{~Sv}$ \\
\hline $\mathrm{BC}$ across $28.5^{\circ} \mathrm{S}$ & $5.12 \pm 0.04 \mathrm{~Sv}$ & $-0.01 \pm 0.02 \mathrm{~Sv}$ & $+0.02 \pm 0.03 \mathrm{~Sv}$ \\
\hline
\end{tabular}

Table 6: Two-sided $t$-test values $(p<0.05)$ between the MCA/LIA and the LM intervals. The significance test was performed between time series of the monthly anomalies, with no low-pass filtering. $p$-values smaller than the 0.05 significance level (i.e., $H=1$ ) are marked in bold, while $p$-values greater than 0.05 (i.e., $\mathrm{H}=0$ ) appear in gray.

\begin{tabular}{|c|c|c|}
\hline Time Series & MCA-LM $p$-Value & LIA-LM $p$-Value \\
\hline $\mathrm{SBL}$ at $25 \mathrm{~m}$ & $1.06 \times 10^{-7}$ & $1.19 \times 10^{-7}$ \\
\hline SBL at $100 \mathrm{~m}$ & $2.18 \times 10^{-6}$ & $2.04 \times 10^{-10}$ \\
\hline total sSEC $\left(9.5^{\circ}-24.5^{\circ} \mathrm{S}\right)$ & 0.0992 & 0.0244 \\
\hline cSEC/eSEC $\left(3.5^{\circ}-8.5^{\circ} \mathrm{S}\right)$ & $8.07 \times 10^{-5}$ & $2.68 \times 10^{-6}$ \\
\hline NBC across $1.5^{\circ} \mathrm{S}$ & $6.63 \times 10^{-4}$ & $8.84 \times 10^{-6}$ \\
\hline northern sSEC $\left(9.5^{\circ}-15.5^{\circ} \mathrm{S}\right)$ & 0.0150 & 0.0043 \\
\hline NBUC across $6.5^{\circ} \mathrm{S}$ & 0.0076 & $1.63 \times 10^{-4}$ \\
\hline southern sSEC $\left(16.5^{\circ}-19.5^{\circ} \mathrm{S}\right)$ & 0.0448 & 0.0860 \\
\hline $\mathrm{BC}$ across $22.5^{\circ} \mathrm{S}$ & 0.0011 & $2.11 \times 10^{-6}$ \\
\hline $\mathrm{BC}$ across $28.5^{\circ} \mathrm{S}$ & 0.0091 & 0.0014 \\
\hline
\end{tabular}




\subsubsection{Wind Stress Curl field}

The wind field distribution also confirmed the observed differences in the SAO circulation patterns between the MCA and LIA (Figure 20). The wind stress is a driving agent of ocean currents, but it is the horizontal gradient rather than the absolute strength that mostly matters: the large-scale wind stress curl, which is, in general, the major forcing mechanism of the upper ocean Goni and Wainer (2001); Cai (2006). Positive, anticyclonic WSC provides the torque that drives the SASG circulation.

The WSC anomalies for the MCA are shown in Figure 20a. Predominantly negative anomalies at the region of positive WSC, south of the zero WSC line, pointed to a weakened subtropical gyre circulation. North of the zero WSC line, the negative anomalies suggested an intensification of the cyclonic circulation in the region, which is consistent with the strengthening of the northern SSEC, which feeds into the NBUC (Figures 17b and 19k,d). For the LIA, Figure 20b shows positive WSC anomalies roughly along the path of the SASG boundary currents (BeC-sSEC-BC-SAC), reinforcing the local positive WSC and strengthening the subtropical gyre circulation. North of the zero WSC line, the positive WSC anomalies relate to a weakened northern sSEC, instead.

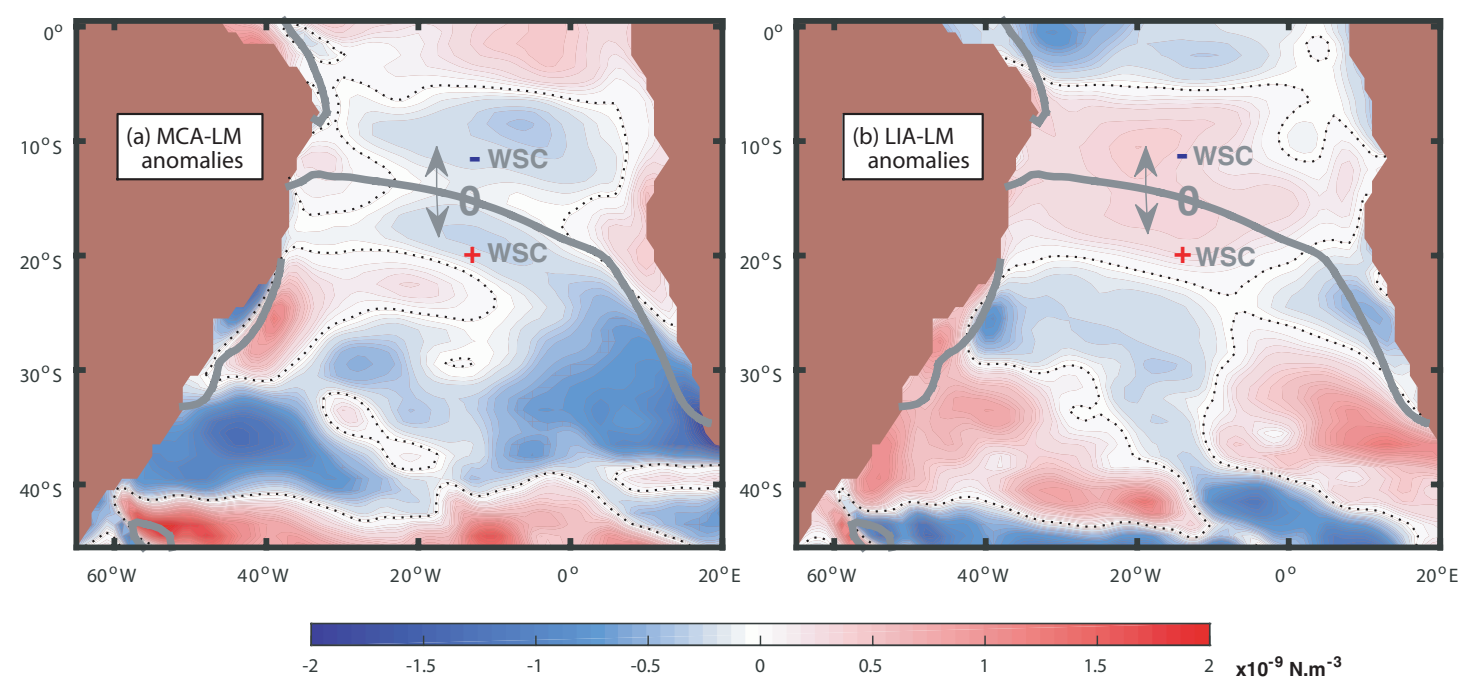

Figure 20 Wind stress curl (WSC) anomalies $\left(\times 10^{-9} \mathrm{~N} \cdot \mathrm{m}^{-3}\right)$ with respect to the whole-LM base period (850-1849). (a) For the MCA, between 950 and 1249, and (b) for the LIA, between 1400 and 1699. The black dashed contours mark the division between positive (red) and negative (blue) anomalies. The gray solid line denotes the LM-mean zero wind stress curl line, north (south) of which the WSC is negative (positive), i.e., cyclonic (anticyclonic). 


\subsection{Conclusions and Discussion}

The circulation pattern associated with the South Atlantic surface boundary current system was investigated for the LM, focusing on its response to the warmMCA and the cold-LIA periods. We examined the along-coast distribution of the SSEC westward flow coming across the SAO basin and bifurcating between the downstream northern and southern circulation regimes. Through the simulation results from the CESM-LME experiment, signals in the SST field suggested a more persistent and far-reaching northward propagation of anomalies along the boundary currents' path during the MCA when compared to the LIA. The circulation field derived from the horizontal velocities revealed overall northward anomalies off the SA coast during the MCA, with increased northern SSEC and NBUC transports, and southward anomalies during the LIA, with increased southern SSEC and BC transports. The SBL accompanies these changes in WBCs transport, as these have an indirect relationship: during the MCA (LIA), the increased northward NBUC (southward BC) transport pushed the SBL to the south (north). Moreover, wind field anomalies indicated that the anticyclonic subtropical WSC, which drives the SASG circulation, was in general weaker during the MCA, while stronger during the LIA.

Together, these results yield a contrasting scenario between the LM-warm and -cold periods. While during the MCA, the flow arriving at the western boundary was preferably directed northward within the AMOC upper limb, during the LIA, a more vigorous SASG circulation was observed, with the increased southward transport.

The MCA/LIA circulation differences described here were in agreement with other large-scale changes reported in the literature for these anomalous periods. During the MCA, the increased northward-NBUC transport and southerly SBL related to studies that pointed to an enhanced AMOC (Trouet et al., 2009) and northern Intertropical Convergence Zone (ITCZ) (Haug et al., 2001); while during the LIA, the increased southward-BC transport and northerly SBL provided compelling evidence for a weaker (Lund et al., 2006) and more saline (Lund and Curry, 2006) Gulf Stream, which acted to stabilize the North Atlantic Deep Water formation (Vellinga et al., 2002; Vellinga and Wu, 2004; Keigwin and Boyle, 2000), 
leading to a weakened AMOC (Trouet et al., 2012), with reduced heat transport and southerly ITCZ (Lund and Curry, 2006; Haug et al., 2001). The position of the ITCZ responds to meridionally-asymmetrical heating changes, shifting toward the warmer hemisphere (Kang et al., 2008; Stevenson et al., 2016), so that reduced northward surface heat transport cools the North Atlantic relative to the South Atlantic, resulting in increased surface pressure over the North Atlantic and a southward shift of the ITCZ (Hastenrath and Greischar, 1993).

Even though one might intuitively expect a stronger AMOC taking place since the Twentieth Century warming, in analogy to the warm-MCA period, studies suggested that the AMOC has weakened substantially over the past decades (Srokosz and Bryden, 2015) and even the past century, displaying a 1975-present decline trend that is unprecedented in the whole LM (Rahmstorf et al., 2015). It has been suggested that this weakening occurred as an abrupt shift towards the end of the LIA or as a more gradual decline over the past 150 years (Thornalley et al., 2018). The possible reasons why the AMOC has not swung back to usual warm-intensified conditions instead of continuing to decline after the LIA are still subject to investigation.

Juckes et al. (2007); Mann et al. (2009), and Ljungqvist et al. (2010) agreed that in general, the warmest temperatures of the past millennium occurred around the 11th Century, and the coolest at some point during the 16th-19th Centuries. The MCA and LIA are well marked in proxy records, but reliable only for the Northern Hemisphere (Solomon et al., 2007; Juckes et al., 2007; Mann et al., 2008, 2009; Ljungqvist et al., 2010; PAGES 2k-PMIP3 Group, 2015; Smerdon and Pollack, 2016). Therefore, there is much to be uncovered about the behavior of the Southern Hemisphere, in particular the South Atlantic Ocean, during these two climatic periods. Very little is known about the Southern Hemisphere climate in the $L M$, and still, the existing knowledge is over continental regions.

The LM was a stable period, and on average, the magnitude of variations was small. Actually, the coupled ocean response in the South Atlantic for the LM had no apparent trends, although it was sensitive to the warm and cold climate oscillations of the MCA and the LIA.

The western boundary current system off the SA coast was a key re- 
gion for diagnosing variations of the AMOC and the SASG. Anomalies generated around the southern boundary of the SASG, for example, are advected equatorward through SAO boundary currents up to the SA coast, where the flow is then distributed between both of these circulation regimes. Therefore, the SSEC bifurcation region is a crucial point that separates the large-scale subtropical and meridional overturning circulations.

The AMOC sets the characteristics of the climate system at decadal and longer time scales through its heat and freshwater transports (Buckley and Marshall, 2016), and the SAO provides the gateway by which the AMOC connects with the global ocean. In view of that, being able to associate past climate extremes with changes in SAO circulation pathways is necessary for improved understanding of the range of possible future climate changes at the basin-scale. As climate change extends far beyond the rise in global mean temperatures, the results presented here propose possible responses of the SAO surface boundary current system to abrupt climatic variations.

* The article version of this work is published online as:

Marcello, F., I. Wainer, P. R. Gent, B. L. Otto-Bliesner, and E. C. Brady (2019). South Atlantic Surface Boundary Current System during the Last Millennium in the CESMLME: The Medieval Climate Anomaly and Little Ice Age. Geosciences 9(7), 299. https: //doi.org/10.3390/geosciences9070299. 


\section{Upper-limb pathways of the South Atlantic Overturning Circulation in the CESM1-LE: up- to downstream projected changes}

\subsection{Introductory remarks}

Within the framework of the Global Overturning Circulation, the South Atlantic provides the means through which interbasin waters return to the North Atlantic along shallow pathways to feed deepwater formation, that is then exported back in greater depth to the South Atlantic, from where it is distributed across the Southern Ocean, Indian and Pacific basins along deep pathways of both the upper (or mid-depth) and the lower global overturning cells (Talley, 2013; Cessi, 2019).

The process of high latitude convection giving rise to North Atlantic Deep Water (NADW) as the descending branch of the upper primary overturning cell is extremely sensitive to the quality of waters reaching the subpolar North Atlantic, which might impel or inhibit deepwater formation, depending on a delicate balance of temperature and salinity (Stommel, 1961; Stocker and Wright, 1991; Rahmstorf, 1996). These conditions are controlled by a complicated advection feedback working on the scale of the entire Atlantic under the primary influence of either ice loss freshwater discharge or warming associated with increased atmospheric $\mathrm{CO}_{2}$ - which may cause freshwater convergence/divergence to the convection regions, resulting in a positive or negative feedback and therefore controlling overall overturning stability (Weijer et al., 2019 and references therein).

Nevertheless, besides being sensitive to local-freshwater and global-heat perturbations, overturning strength can also be influenced by variations in farther upstream circulation processes and wind-driven gyre exchanges (Wood et al., 2019; Evans et al., 2017; Jamet et al., 2020). Both changes in the amount of contributions from the Indian 'warm water' or the Pacific 'cold water' routes (Gordon, 1985, 1986 and Rintoul, 1991, respectively) - the two contrasting main sources for the near-surface upper limb flow of the Atlantic Meridional Overturning Circulation (AMOC) - , or changes in how these water masses are circulated and transformed within the South Atlantic itself, might influence deepwater convection and therefore 
affect overturning variability and strength (Garzoli and Matano, 2011; Biastoch et al., 2008; Leroux et al., 2018; Speich et al., 2007).

In fact, changes in large-scale overturning and gyre circulations are fundamentally linked - despite their specific dynamical connections remain obscure; with buoyancy forcing driving gyre circulation and wind-driven momentum forcing generating overturning motion, and with their coordinated response to both these forcings being crucial to understand variations in poleward oceanic heat transport (Yeager, 2015).

As the global overturning is centered in the Atlantic basin, research highlights are often focused on the AMOC, more precisely its Northern Hemisphere counterpart. Thus, with respect to the current knowledge about the AMOC state, a gradual AMOC weakening had been observed from 2004 to 2017 at $26^{\circ} \mathrm{N}$ (Smeed) et al., 2014, 2018), raising speculations on whether this short recorded trend was part of a long-term decline induced by anthropogenic warming (Caesar et al., 2018) or whether it was linked to natural multidecadal variability (Roberts et al., 2014; Worthington et al., 2021), or even both (Srokosz and Bryden, 2015; Jackson et al., 2016; Collins et al., 2013).

Recent updates on the observed AMOC strength at $26^{\circ} \mathrm{N}$ have now revealed that it is no longer weakening, although it can't yet be determined if the AMOC might be recovering - since there is no statistically significant increase since its low period in 2010, and the recent transport is not above the long-term mean (Moat et al., 2020).

Modeling and paleoceanographic studies suggest that the AMOC has been declining for the last 150 years (Caesar et al., 2018; Thornalley et al., 2018) and that the modern AMOC slowdown is unprecedented in the past millennium (Rahmstorf et al., 2015; Caesar et al., 2021), although recent observation-based fingerprints indicate that the AMOC has actually lost its stability along the last century, coming close to a critical transition - instead of having been subject to natural fluctuations or a linear response to global warming (Boers, 2021). Actually, it has been implied that the current fast rate of greenhouse gas emissions has been pushing the AMOC into an unsafe and unexpected operating space, leading to a so-called rate-induced tipping (Lohmann and Ditlevsen, 2021) — where an AMOC 
collapse can be induced even by small amplitude changes in forcing, provided that these changes happen in a fast enough pace.

The latest Intergovernmental Panel on Climate Change (IPCC) Assessment Report (Masson-Delmotte et al., 2021) states with medium confidence that the AMOC was relatively stable during the past 8,000 years, but there is low confidence concerning its decline during the 20th century; and while there is high confidence with respect to its directly observed weakening so far, it actually "cannot be distinguished between decadal-scale variability or a long-term trend" (Gulev et al., 2021).

It remains uncertain whether state-of-the-art climate models are biased to overstabilize the AMOC by underestimating freshwater transports (Liu et al., 2017; Boers, 2021), or even whether the AMOC response to anthropogenic forcing has been overestimated by climate models with weak aerosol forcing - which is understood to offset GHG-induced AMOC weakening (Menary et al., 2020), but in turn might act reinforcing it over future decades since anthropogenic aerosol emissions are projected to continually decline in response to pollution control policies (Hassan et al., 2021).

Fundamental links among the factors that might affect past-to-future AMOC stability are still being pursued, as for instance a recent study by Goes et al. (2019) which demonstrates that AMOC strength actually might not have a significant effect on AMOC stability - differently than what was expected based on previous studies. Simulations on how the AMOC may respond to future climate change are subject to substantial intermodel spread (Cheng et al., 2013; Collins et al., 2013; Weijer et al., 2020), also leading to uncertainties with respect to the range and magnitude of its cascading global impacts (Bellomo et al., 2021).

Reality is that the AMOC is a highly non-linear system (Rahmstorf, 2000) and its stability issue along with its response to human-induced global warming are far from being fully resolved (Zhu et al., 2015; Srokosz et al., 2021; Jackson and Wood, 2018), with considerable uncertainties as to where the current climate state places the AMOC along the range of stability thresholds (Weijer et al., 2019). The science community is hence left with the question of whether the ongoing AMOC weakening is faded to be exacerbated, leading the system to a new equilibrium 
state, or if it is "merely" part of a more subtle transient weakening, destined to eventually fully recover (Li et al., 2021).

Still, the majority of our current generation climate models project a future AMOC decline. The latest IPCC Special Report on the ocean and cryosphere stated that "The AMOC is projected to weaken in the 21 st century under all RCPs (very likely)" (Pörtner et al., 2019), while the newly released IPCC Sixth Assessment Report analogously states the same for all Shared Socioeconomic Pathways (SSPS) (very likely) (Fox-Kemper et al., 2021), with stronger AMOC decline depicted by CMIP6 models when compared to those of CMIP5 - and with a surprising insensitivity of this response to differing scenarios at least up to 2060 (Weijer et al., 2020).

The projected AMOC weakening is still generally attributed to the effects of increased greenhouse gases as well as to a range of other natural and anthropogenic external forcings (Menary et al., 2020). Since overall model projections are qualitatively similar (despite substantial quantitative differences), Fox-Kemper et al. (2021) suggest that an "AMOC decline may be governed by large-scale constraints independent of the details of the models"; and, finally, the report concludes only with medium confidence that an abrupt AMOC collapse will not occur before 2100.

While hypothesis can be drawn from AMOC observations in the North Atlantic, South Atlantic moored observations at 11오 (Hummels et al., 2015) and 34오 (Kersale et al., 2020; Meinen et al., 2018) "are currently too short to permit robust conclusions about changes" (Fox-Kemper et al., 2021).

Beyond the AMOC state, systematic changes have been reported in upper ocean gyre circulations. More specifically, in the so-called Southern Hemisphere supergyre (SHSG) (Ridgway and Dunn, 2007) - an interbasin feature which represents the connectivity between all three southern subtropical gyres — linking the South Pacific, South Atlantic and Indian oceans.

Since the beginning of the 21st century, the atmospheric circulation response to human-induced climate change and its consequent impacts on the upper ocean circulation have gained more attention, featuring the combined effects of ozone depletion and increasing $\mathrm{CO}_{2}$ (Kushner et al., 2001; Thompson 
and Solomon, 2002; Shindell and Schmidt, 2004; Saenko et al., 2005; Son et al., 2009; Polvani et al., 2011; Thompson et al., 2011). These act to shift the Southern Annular Mode (SAM) towards its positive polarity, ultimately translating into a spin-up and poleward expansion of the SHSG (Cai et al., 2005; Cai, 2006; Roemmich, 2007; Li et al., 2013; Wang et al., 2014), along with warming of subtropical western boundary currents (Wu et al., 2012). Studies reporting regional southern subtropical gyre changes have mainly focused on the South Pacific (Roemmich et al., 2007, 2016; Zhang et al., 2013; Zhai et al., 2014; Zhang and Qu, 2015; Hu et al., 2015), rather than on the South Atlantic and Indian basins (Alory et al., 2007; Biastoch et al., 2009; Rouault et al., 2009; Beal et al., 2011; Durgadoo et al., 2013).

With ozone recovery (after 2000, when Montreal Protocol effects reached stabilization), but continued rising $\mathrm{CO}_{2}$, the Southern Hemisphere extratropical circulation response to anthropogenic climate change became uncertain - as the ozone effect started to act in the opposite direction (Arblaster et al., 2011). This outcome is further complicated by chaotic natural climate variability - which might reinforce or weaken ongoing trends, or either combine to even out dominant changes (Seager and Simpson, 2016).

Nevertheless, more recent studies continue to describe ongoing Southern Hemisphere ocean circulation changes in association to shifted wind-patterns (Yang et al., 2016, 2020; Qu et al., 2019; Pontes et al., 2016; Marcello et al., 2018; Rühs et al., 2019; Drouin et al., 2021; Sen Gupta et al., 2016; Gupta et al., 2021), probably reflecting a domination of the increasing $\mathrm{CO}_{2}$ effect. Actually, a new study from Goyal et al. (2021) concludes that the poleward intensification of SH surface westerlies is projected to continue in the future under a high emission scenario - in spite of the projected stratospheric ozone recovery, once future trends are dominated by the GHG forcing.

In response to a poleward shift of the multi-basin scale wind stress curl - due to anthropogenic global warming, the IPCC Sixth Assessment Report concludes with medium confidence that western boundary currents and subtropical gyres have shifted poleward since 1993; changes in WBCs strength are "more complex and inconsistent across regions" - since they are not only dependent on 
wind stress forcing (Fox-Kemper et al., 2021; Gulev et al., 2021).

The potential slowdown of the modern and future AMOC is likely to extend all the way into the South Atlantic (Zhu and Liu, 2020), while, at the same time, MOC anomalies observed in the North Atlantic can either be of local origin or originate upstream in the South Atlantic and beyond (Blunden and Arndt, 2020; Garzoli et al., 2013). In a time when human activity is forcing significant changes to the Earth system, improved understanding of the extension of an AMOC slowdown as well as of its remotely formed anomalies are now needed, being the next step "to resolve which components and pathways of the AMOC have altered, how, and why" (Caesar et al., 2021).

The majority of efforts to understand the AMOC dynamics and variability are still focused on the North Atlantic, generally disregarding the upstream course of the northward advection of waters through the upper limb flow since its origins in the southern South Atlantic, encompassing both the meridional and zonal components of the overturning circulation surface pathways.

Beyond considering changes in the inflow of Pacific and Indian waters to the South Atlantic, it is imperative to ponder how these potential flux changes have been and might be communicated and distributed by South Atlantic intrinsic circulation processes, particularly those involved in the wind-driven, anticyclonic South Atlantic subtropical gyre circulation. Conversely, it is equally desired to assess how changes in high-latitude overturning and hemispheric-scale atmospheric forcing are to be reflected along main circulation routes of the global ocean.

This chapter investigates the direction of projected changes in the upper limb pathways of the South Atlantic overturning circulation as expressed by the simulation results of the CESM1-LE under the most aggressive, yet most realistic, scenario in assumed fossil fuel use for global climate models (Schwalm et al., 2020) - the RCP8.5.

The CESM1-LE ensemble mean is used to represent the climate system forced response, which points to a series of transmitted long-term signals, yielding a spatio-temporal pattern of projected changes in South Atlantic circulations involving both the SASG and the AMOC upper limb.

Therefore, our main goal is to illustrate a possible future evolution path 
for the large-scale circulation of the South Atlantic and its surroundings which are linked to AMOC and SASG pathways, as according to the emissions trajectory and consequent radiative forcing (i.e., the additional amount of energy in Earth's climate system by 2100 , relative to pre-industrial levels) depicted by the RCP 8.5 scenario when applied to the multiple simulations of a single-model large ensemble, after which their common forced response has been extracted.

\subsection{Data and Methods}

\section{The Community Earth System Model Large Ensemble}

The numerical results of the National Center for Atmospheric Research (NCAR) Community Earth System Model Large Ensemble Experiment (CESM1LE) are used (Kay et al., 2015). The CESM1-LE represents a community resource for studying climate change in the presence of internal climate variability. It includes a 40-member ensemble of fully-coupled CESM1 simulations for the period of 19202100. Each member is subject to the same radiative forcing scenario (historical up to 2005 and RCP8.5 thereafter), but begins from a slightly different initial atmospheric state (created by randomly perturbing temperatures at the level of round-off error).

All the simulations are derived from the CMIP5 version of the CESM, documented in Hurrell et al. (2013). The horizontal resolution is at approximately $1^{\circ}$ in all model components. The experiment includes a multi-century control integration followed by a transient integration (ensemble member 1) which started at year 402 of the control and was integrated for 251 years under historical forcing (1850-2005). Next, the protocol for running projections in CMIP5 models is applied. The projection scenario used in these runs was established by the IPCC, and is referred to as the Representative Concentration Pathway $(\mathbb{R C P}) 8.5$ scenario, for 2006-2100. The additional ensemble members were initialized from ensemble member 1 at January 1920 with the round-off level perturbations applied to the air temperature field.

To make meaningful interpretations about past to future South Atlantic overturning circulation trends, a deeper understanding of the competing roles of natural climatic variability and forced change is required. Since different models 
have different sensitivities to an identical set of radiative forcings (Deser et al., 2014; Solomon et al., 2007), they end up generating exclusive sequences of internal variability - making this inherent uncertainty undersampled by multimodel ensembles. Here, the use of a single-model "large ensemble" eliminates structural differences inherent to multi-model ensembles, allowing the spread across the ensemble to be entirely attributed to the internal variability of the modeled climate system.

For each ensemble member, temporal trends in any variable can be separated into two parts: (1) the forced trend that is common across all ensembles, and (2) the unforced, or internal trend, that occurs only in that ensemble member. The ensemble mean averages out natural climate variability and thus represents the forced response.

The results shown here represent the ensemble mean of the 40 -full-forcing members for the historical period (1920-2005, 20TR) and future climate change projections (2006-2100, RCP8.5).

\section{Data Analysis}

Prior to averaging CESM-LE members, annual means were obtained from the original monthly mean data set in order to remove higher-frequency variability in which we are not particularly interested in. Since the focus of this study is on the upper-ocean pathways of South Atlantic circulations, nearly all analysis concern the upper 985-m depth, using the original model depth levels for vertical integrations. Vertical profiles are shown up to 3-5 $\mathrm{km}$ for reference.

The volume transports time series were obtained by horizontally and vertically integrating the zonal (u) or meridional (v) velocities along model grid cells. When calculating positive (negative) velocities, i.e. northward or eastward (southward or westward), only positive (negative) values are considered, in order to avoid adjacent counterflow interference, with the exception of MOC streamfunction time series. Transport time series are derived for individual zonal/meridional coordinates (transects) or for zonal/meridional ranges (boxes); in the latter case, the transport time series corresponding to each coordinate within the selected range was first obtained, then these individual time series were averaged to 
represent the mean transport across a wider range only if they were considered to have very similar low-frequency variability and, mainly, long-term trends. Thus, the transport time series associated with boxes express low-frequency signals and changes that are common to all transects which compose the respective box, for simplification.

All volume transports are vertically integrated up to $985-\mathrm{m}$, with the exception of MOC streamfunction time series, which are vertically integrated up to the lower limit of the northward AMOC upper limb in each time step (i.e., up to the depth of transition between northward and southward velocities). The absolute magnitude of the horizontal velocities (Figures 21k, d) is defined by $\sqrt{u^{2}+v^{2}}$, applied to each grid-point of the domain.

Anomalies are derived with respect to the 1920-1970 base period, corresponding to the first 50 years of the historical period (20TR), when climate change signals were relatively modest. Zonal-meridional maps and meridional-depth profiles of "linear trends" correspond to the total trend with respect to the 1920-2100 period. 1920-1970 / 2080-2100 differences are discussed, aiming at demonstrating the contrast between a period before greenhouse gas emissions became to increase exponentially and a projected period in the late 21 st century which reflects the future changes expressed by the high-end RCP8.5 scenario. For the Empirical Orthogonal Function (EOF) analysis, linearly detrended anomalies with respect to the 1920-1970 base period are used (the linear trends were removed from each grid-point prior to the EOF analysis). The EOF analysis is used to extract the dominant pattern of variability in the zonal flow field of the SASG.

\subsection{Results}

\subsubsection{Characterization of the General Mean Circulation Field}

The upper 985-m, 1920-2100 mean zonal and meridional velocities are individually shown in Figures 21a, b, respectively, with transects and boxes (identified by numbers and colors) indicating where volume transport time series were derived. These correspond to the numbers/colors referenced in Figures 22, 23 , 24, 25, 26 and 27, which all show the vertical profiles and volume transport time 
series associated with every transect/box illustrated in Figures $\mathbf{2 1} \mathbf{a}, \mathbf{b}$. From here on, numbers succeeding referenced figures are attributed to the numbered transects/boxes or vertical profiles and/or time series corresponding to the respective figure (for example, "Figure 21 - 1" refers to the green box ' 1 ' in that figure).

A general picture of the mean horizontal flow field, encompassing both zonal and meridional current velocities, is provided by Figure $21 \mathrm{c}$ - where the absolute magnitude of the horizontal velocities is shown as background colors and both the flow direction and intensity are represented by the superposing vectors. Velocity vectors are further distinguished between regions where the mean absolute magnitude of the horizontal velocities is above (black vectors) or below (gray vectors, scaled by a factor of 50 ) the mean value $\left(0.035 \mathrm{~m} . \mathrm{s}^{-1}\right)$.

The regions of greater flow intensity as well as those with broader and diffuse pathways are clearly signalized. The main outstanding pathways are mostly related to important circulation features that take part in the global overturning circulation. For the description given hereafter, the reader who is not familiar with South Atlantic large-scale circulations and its ocean currents is referred to Figure 3 from Stramma and England (1999), to aid visual interpretation.

Following up- to downstream flow direction, the upper limb pathways which compose the South Atlantic overturning circulation begin in the region of the Drake Passage (DP), prior to which a strong zonal component is observed in the Southern Ocean Pacific sector (Figures 21a - 1 and 21 c). Between the southern tip of South America and the northern tip of the Antarctic Peninsula, strong zonal and meridional components mark the mean circulation field (Figures 21a - 5, 6; 21b - 7;21c).

In general, part of the DP flow turns northward forming the Malvinas Current (IMC) (Figure 21b - 7, 8) and the other part continues to flow eastward along the Antarctic Circumpolar Current (ACC) (Peterson and Stramma, 1991; Schmid, 2014) (e.g. Figure 21a - 2, 3). Past the DP, the vast eastward circulation belt crossing the Atlantic basin from the eastern South America towards western Africa comprises a complicated interplay between the South Atlantic Current (SAC) to the north, delimiting the southern boundary of the SASG, and the wide circumnavigating ACC to the south (Boebel et al., 1999; Speich et al., 2001), with 
continuous exchange between these currents occurring at least up to $\sim 35^{\circ} \mathrm{W}$ (Rühs et al., 2019; Drouin and Lozier, 2019; Boebel et al., 1999).

The portion of the DP flow that takes part in the overturning circulation, actually entering the South Atlantic basin, is known as the "cold water route" (Macdonald, 1998; Sloyan and Rintoul, 2001; Speich et al., 2001; You, 2002; Friocourt et al., 2005). It follows the MC to the north until meeting the southern end of the western boundary Brazil Current (BC), where the Brazil-Malvinas Confluence (BMC) takes place. From both these converging currents, the $S A C$ is originated (Figure 21a - 11, the eastward (red) core located in between the westward (blue) components of the $B C$ and $M C$ - indicated by transects 10 and 9 , respectively).

Overall, besides partly escaping to the ACC (Rodrigues et al., 2010), the SAC either crosses the South Atlantic basin reaching the Benguela Current $(\mathrm{BeC})$ - turning northward and recirculating along the outer edge of the subtropical gyre -, or entrains the subtropical gyre via interior pathways that connect it directly to the southern branch of the South Equatorial Current (SSEC) flowing westward along the northern limb of the gyre (Schmid, 2014). This is expected, considering the D-like subtropical gyre shape (reflected in Figures 21a-d), dictated by its controlling dynamics - the Coriolis-forced western intensification and consequent eastern diffusing.

At the eastern South Atlantic boundary, a strong westward component of the Agulhas leakage (AL) is found (Figure 21a - 12), extending from the upstream southward Agulhas Current (AC) outlining the African east coast (Figure 21b). The AL contribution to the overturning circulation is termed as the "warm water route" (Gordon, 1985, 1986). South of the African continent, mixing of cold and warm water routes occur (Drouin and Lozier, 2019). AL and recirculated SAC end up entering the $\mathrm{BeC}$-sSEC system, which delineate the sluggish curvilinear eastern-to-northern boundaries of the SASG.

The total westward flow crossing the South Atlantic basin and eventually spreading out between the northern and southern extensions of the South American coast (depicted by background blue colors between $\sim 10^{\circ}-40^{\circ} S$ in Figure 21 a) comprises mostly the sSEC - considered the zonal extension of the $\mathrm{BeC}$ that defines the northern limb of the SASG (Richardson, 2007). 

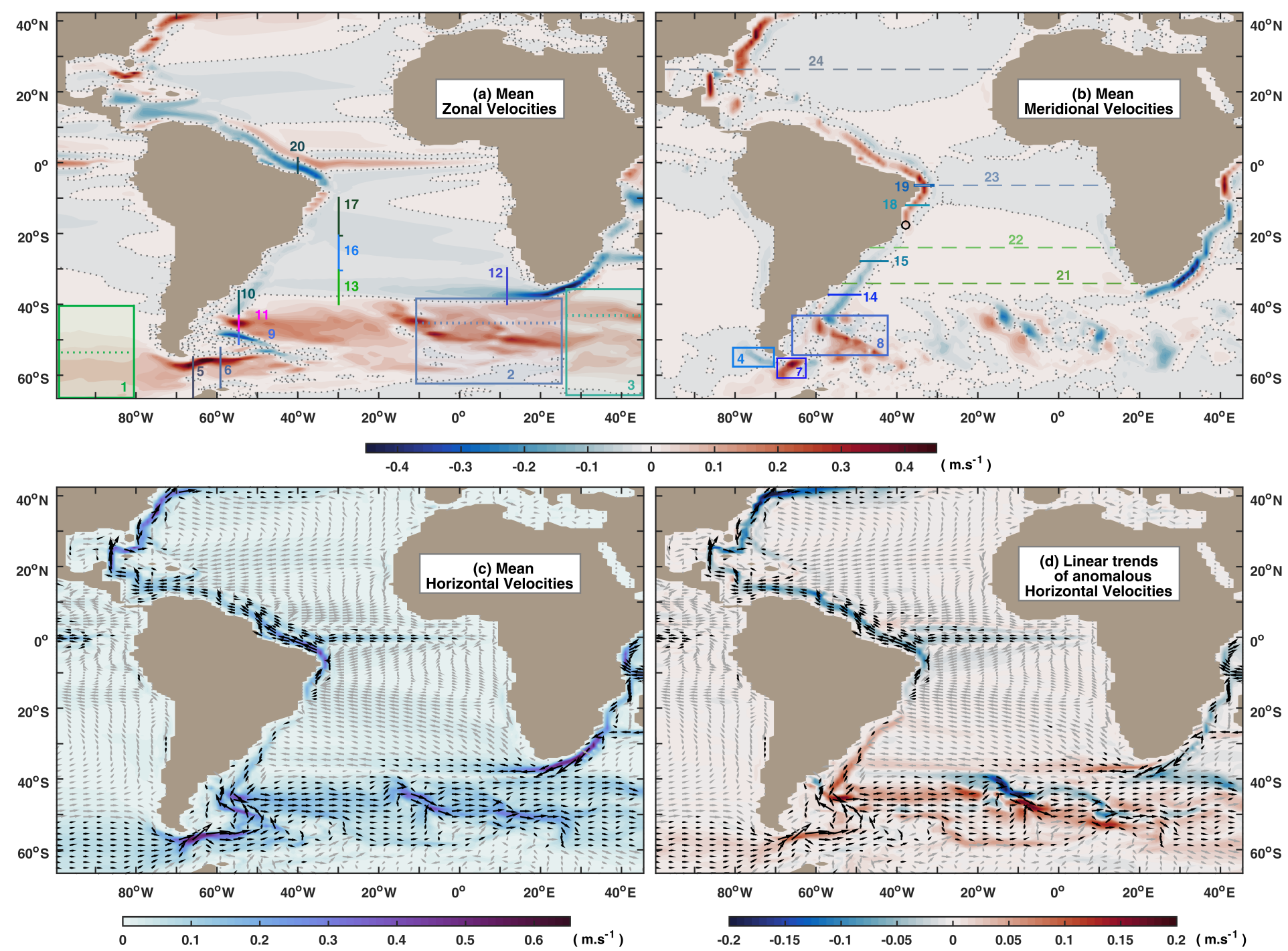

Figure 21 | Horizontal velocities - mean and trends | Mean 1920-2100, upper 985-m, zonal (a) and meridional (b) velocity fields. Red represents positive (eastward/northward) velocities, while blue represents negative (westward/southward) velocities; dotted gray lines indicate the zero zonal/meridional velocity line. The regions where zonal and meridional volume transports were derived are shown in varied colors (boxes and transects), as a reference for the vertical profiles and time series in Figures 22, 23, 24, 25, and 26- with corresponding colors and numbers. The black circle on (b) represents the mean latitude where the southern branch of the South Equatorial Current bifurcates. (c) The mean absolute magnitude of the horizontal velocity field is represented by the background colors, superposed by zonal and meridional velocity vectors, giving a perspective of the mean flow intensity/magnitude and direction. Black (gray) vectors are plotted over regions where the absolute velocity magnitude is above (below) the mean value $\left(0.035 \mathrm{~m} . \mathrm{s}^{-1}\right)$, for reference; gray vectors are scaled by a factor of 50, in comparison with black vectors. (d) 19202100 total trends of anomalous horizontal velocities (of their absolute magnitude, as background colors); the anomalies are with respect to the 1920-1970 base period. Red (blue) indicates regions where velocities magnitude tend to increase (decrease), i.e. with positive (negative) trends. Superposed on background colors are the same 1920-2100 mean vectors from (c), for reference.

The general SEC has been further classified into several other branches (a brief yet more detailed description of the SEC excursions is provided by Marcello 
et al. (2019) - reproduced on Section 4.3.2), however, relevant to this study is the fact that these other SEC branches all originate from a portion of the SEC that is described by Stramma (1991) to turn northward near $30^{\circ} \mathrm{W}$, merging into the equatorial current system and eventually branching into central and northern SEC portions, mainly.

In other words, the remaining SEC branches flowing north of the SSEC do not reach the western boundary and therefore do not bifurcate into northand southward low-latitude western boundary currents, neither are considered to define the northern boundary of the SASG. An inflow from the central SEC is received north of $\sim 5^{\circ} S$ at the western boundary (Schott et al., 1995), however, this contribution feeds directly into the northward component along the coast (more specifically, the downstream North Brazil Current - NBC, Figure 21a - 20), while the SSEC is associated with the origins of both the northward North Brazil Undercurrent (NBUC) and the southward Brazil Current (BC) (Figure 21b - 18, 19 and 15,14 , respectively).

While the numerous northern counterparts of the SEC have been well described and documented (see for instance Figures 2-4 from Stramma and England, 1999 and Figures on pages 289, 292 and 294 from Stramma and Schott, 1999), the wide yet single sSEC branch has long been left without a comprehensive characterization of its structure and its links to the western boundary which directly affect and modulate the dynamics of its bifurcated meridional flows.

Evidence for a multi-banded SSEC can be inferred from the anomalous sSEC pattern shown in Marcello et al. (2019) for the last millennium, from the CESM-LME simulation results (Figure 17), and was also recently provided by Luko et al. (2021) (summarized and clearly illustrated in their Figure 15), who characterize the SSEC mean state using altimetry satellite and reanalysis data. Still, their characterization show a southernmost SSEC band occurring north of $25^{\circ} \mathrm{S}$, in accordance to the definition provided by Stramma (1991) - of the SSEC as a flow between $10^{\circ} \mathrm{S}$ and $25^{\circ} \mathrm{S}$.

The CESM-LE, however, simulates a southern westward pathway that extends up to $\sim 40^{\circ} \mathrm{S}$, traced back to the $\mathrm{AL}$ at the eastern boundary, as a straight route that feeds directly the BC across the basin (Figure 21a - 12,13). Considering 
this route too vigorous to characterize merely "interior pathways", here it is referred to as a sSEC sub-branch (the southern SSEC); and the SSEC is thus defined as the westward flow between $\sim 10^{\circ}-40^{\circ} \mathrm{S}$, partitioned between southern, central and northern branches (Figure $\mathbf{2 1} \mathbf{a}$ - 13, 16 and 17, respectively). In fact, this partition is chosen primarily in accordance to the differing long-term signals expressed by each of these sub-branches.

Overall, while the southern SSEC appears as a route that is preferably linked to the poleward BC, the northern SSEC seems to contribute most exclusively to the NBUC; and the central SSEC is left as the portion that is more prone to bifurcate - considering an oversimplified visual inspection of the upper 985-m mean layer (Figures 21a-c).

The sSEC bifurcation actually depicts a poleward shift with depth, reflecting the subtropical gyre typical "bowl shape" at its northern boundary. As the SSEC reaches the coast in ever more southern latitudes with increasing depth, the SSEC bifurcation latitude (SBL) is also consecutively pushed to the south (shown in Figure 30). The depth-averaged SBL is indicated by the black circle in Figure 21b.

The diverging western boundary currents which are originated with the bifurcation of the SSEC are each associated with main large-scale circulation features - while the BC closes the subtropical gyre circulation, the NBUC acts as a conduit for the AMOC upper limb return flow towards the subpolar North Atlantic.

Finally, the transbasin transects at $34.5^{\circ} \mathrm{S}, 24.5^{\circ} \mathrm{S}, 6.5^{\circ} \mathrm{S}$ and $26.5^{\circ} \mathrm{N}$ (Figure 21b - 21-24, vertical profiles shown in Figures 27a-d) correspond to the AMOC streamfunction time series (defined by the zonally and vertically integrated meridional velocities across the Atlantic basin and up to the lower limit of the northward upper limb in each time step - Figure 26h - 21a-24a) and also to time series which reflect exclusively the northward transbasin transport (Figure 26 $\mathbf{h}$ $21 b-24 b)$, not accounting for southward flows of any kind.

\subsubsection{Overall Large-Scale Projected Changes}

The 1920-2100 total trends for the absolute magnitude of the horizontal velocity anomalies (with respect to 1920-1970) are shown in Figure 21d as background colors. Superposed are shown the 1920-2100 mean velocity vectors, for 
reference. The mean vectors indicate the direction of the mean flow, while red (blue) background signalizes where current velocities are projected to strengthen (weaken) along 1920-2100, when compared to the 1920-1970 climatology.

The trend map in Figure 21 d serves as a starting point for the presentation of the next results, which each concern specific regions within this domain.

Essentially, increasing current velocity trends are found from upstream of the DP into the South Atlantic via the MC, and then to the east marking a strengthened SAC up to $\sim 20^{\circ} \mathrm{W}$. East of $20^{\circ} \mathrm{W}$, this eastward intensification seems to be deflected southward, leaving a band of negative anomalies immediately to the north of this excursion. Actually, these negative anomalies extend from north of the SAC origins, near $\sim 50^{\circ} \mathrm{W}$ (along $\sim 40^{\circ} \mathrm{S}$ ); while the eastward flow heading to the Indian Ocean, just south of the AL along this same latitude, displays decreasing trends as well.

Moving north in order to track westward-oriented flows, the upstream AC is marked with decreasing trends all along the eastern coast of Africa, however, its portion that leaks directly into the South Atlantic (translating into a strictly westward flow along $\sim 37^{\circ} \mathrm{S}$ ) in turn shows increasing trends that extend up to the opposite side of the basin, building up to the southward BC flow at the western boundary. This westward cross-basin path corresponds to portion of the mean flow that was previously defined here as the southern SSEC; this portion is also the one thought to be connected to the SAC via intra-gyre pathways (Schmid, 2014; Drouin and Lozier, 2019).

By shifting focus to the meridional divergent flows along the western boundary of South America, trends of opposite signs are found straddling the mean SBL region (the black circle in Figure 210). While the poleward BC is projected to strengthen up to its downstream end (thus also contributing to form a stronger SAC), the equatorward NBUC-NBC system exhibits conspicuous negative anomalous trends - expanding downstream towards the subpolar North Atlantic. 


\subsubsection{Drake Passage, ACC and southern SASG}

\section{Contextualization}

The Drake Passage (DP) marks the only latitudinal band in the world's ocean where there is no continental barrier (e.g., Toggweiler and Samuels, 1995). It is the narrowest stretch of water separating the west Antarctic continent, at the northern tip of Palmer Peninsula $\left(62^{\circ} \mathrm{S}\right)$, from the southern tip of South America, the Cape Horn $\left(56^{\circ} \mathrm{S}\right)$. It connects the Atlantic and Pacific Oceans through the strong eastward ACC flow, injecting Pacific waters to the SAO and thus sustaining the GOC through the cold water route (Rintoul, 1991; Rintoul et al., 2001).

Recent Lagrangian studies have suggested a $40 \%$ contribution of the cold water route to the upper limb of the AMOC (Rühs et al., 2019; Drouin and Lozier, 2019), an higher estimate than that of previous studies (Donners and Drijfhout, 2004; Friocourt et al., 2005; Speich et al., 2001).

Even though the total volume transport through the DP (of $\sim 118$ to 173 Sv, Whitworth III, 1983; Orsi et al., 1995; Meredith et al., 2011; Cunningham et al., 2003; Donohue et al., 2016) is known for comprising mostly the wide ACC flow, one can naturally expect that flows associated with the Bellingshausen Sea circulation off the Antarctic Peninsula join the southern ACC flow, while residual flows from the eastward transport along the Tasman Front (TF) - at the southern boundary of the South Pacific subtropical gyre (SPSG), e.g., Hu et al. (2015) - end up joining the northern ACC flow after meeting the South American west coast and sliding southward by following the coastline toward Cape Horn (see Figure 21b - 4, for instance).

These contributions are merged together in order to make it through the DP. From thereon, the total volume transport is further spread and distributed between the circumnavigating ACC continuing eastward and portions which are deflected northward outlining Cape Horn - originating the MC off the South American coast and joining the SAC throughout the southern limb of the SASG.

Studies provide evidence for a varied possibility of water pathways once they have passed the DP, instead of a couple of exclusive routes (Schmid, 2014; Drouin and Lozier, 2019; (Rühs et al., 2019; Boebel et al., 1999). Although the total DP flow primarily remains in the ACC, small fractions are diverted northward in 
different portions of the southern South Atlantic domain. Besides the continuous MC-SAC-BeC-sSEC system (i.e., the "direct" cold water route - Friocourt et al., 2005; Speich et al., 2001), ACC-SAC as well as interior SAC-sSEC entrainment occurs throughout the South Atlantic southern boundary. Moreover, a portion of waters that exit the South Atlantic with the ACC recirculate in the Indian Ocean and eventually merge with the $A L$ (the warm water route) - characterizing an indirect cold water route (Bower et al., 2019; Speich et al., 2007).

For this reason, it is important to take into account individual local and remotely originated portions of the up- to downstream total DP transport in order to obtain insights into the actual distribution of the flow parcels and the signals carried by each of them.

\section{Changes in up- to downstream eastward volume transports along the Southern Ocean}

Before acknowledging the inflow of the DP transport into the SAO, the bulk of the eastward flow prior to the DP (Figure 21a - 1) as well as further downstream leaving the Southern Ocean South Atlantic sector (Figure 21 a - 2, 3) is investigated in Figures 22, 23 and 24. A general description of the results associated with each panel from these three analogous figures is provided below.

The 1920-2100 mean vertical profiles of the zonal velocities averaged for all zonal sections within boxes 1,2 and 3 are shown in panels (a). These averaged profiles are fairly representative of all individual profiles corresponding to the longitudinal range of boxes 1-3. Between boxes 1 and 2 (east of $80^{\circ} \mathrm{W}$ and west of $10^{\circ} \mathrm{W}$ ), the bathymetric and flow configurations exhibit a more heterogeneous spatial structure, and for this reason the eastward flow is not averaged into a box in this region.

The 1920-2100 temporal evolution of the zonal velocity anomalies corresponding to the upper $985-\mathrm{m}$ (right above 1-km) mean layer of panels (a) is shown in panels (b) - as Hovmoller diagrams along the same meridional extension.

The black contours in Figures $23 \mathrm{~b}$ and $\mathbf{2 4} \mathrm{b}$ delimit the region of mean eastward flow field along time (it is the raw, not anomalous, zero zonal velocity line). In Figure 22b, the full y-axis meridional range comprises deviations (anomalies) 
with respect to an eastward mean flow. Hence, for all anomalies in Figure $\mathbf{2 2} \mathbf{b}$ and for the anomalies in between the black contours in Figures $23 \mathbf{b}-\mathbf{2 4} \mathbf{b}$, red points to a strengthened, while blue points to a weakened eastward flow.

(a) Mean $100^{\circ}-80^{\circ} \mathrm{W}, 1920-2100$ Zonal Velocities:

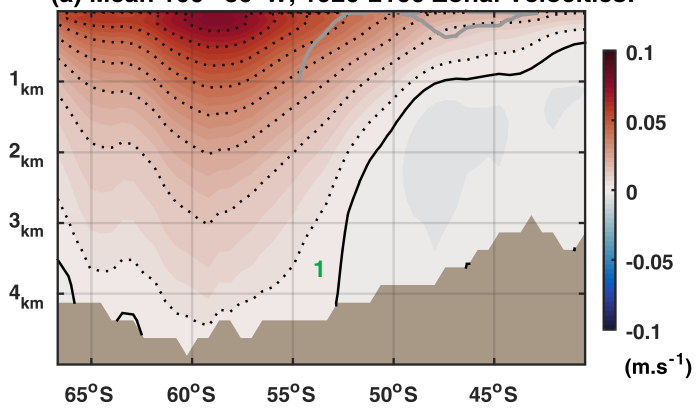

(b) Hovmoller diagram $-100^{\circ}-80^{\circ} \mathrm{W}$, upper $985 \mathrm{~m}$ :

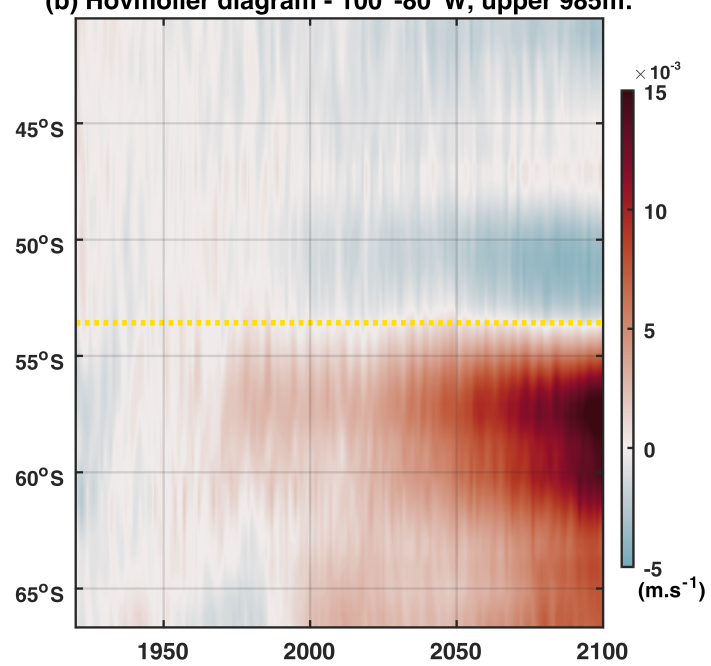

(c) Linear trends of Zonal Velocities [100-80 W]
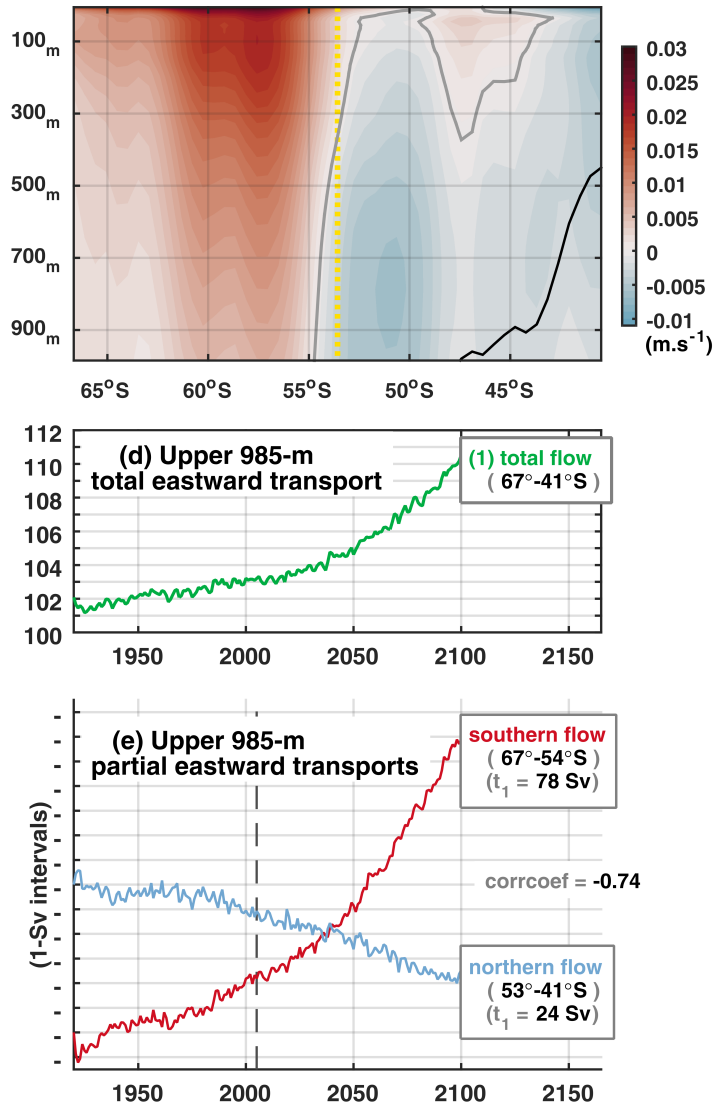

Figure 22 Southern eastward flow prior to the Drake Passage $\left(100^{\circ}-80^{\circ} \mathrm{W}\right) \mid$ Zonal velocities in the southern Pacific sector, averaged over $100^{\circ}-80^{\circ} \mathrm{W}$ (box number 1 in Figure 21a). (a) 19202100 mean vertical profile; red denotes positive (eastward) and blue denotes negative (westward) velocities. The black filled contour represents the zero zonal velocity line; and the black dotted contours indicate zonal velocity lines along values of $4 \times 10^{-3}: 10^{-2}: 74 \times 10^{-3}$. The gray filled contour in the upper 1-km is reproduced from (c), for reference. (b) Hovmoller diagram (upper 985-m mean) of anomalous zonal velocities (1920-1970 base period); red (blue) indicates positive/eastward (negative/westward) anomalies. The yellow dotted line marks the mean latitude of transition between positive and negative anomalies $\left(53.5^{\circ} \mathrm{S}\right)$, in correspondence to the yellow dotted line in (c), used as a reference to derive the southern and northern volume transports in (e). (c) 1920-2100 total trends of anomalous zonal velocities, corresponding to the upper 985-m layer in (a). The gray filled contour marks the transition from negative (blue) to positive (red) trends. The black filled contour corresponds to the upper $985-\mathrm{m}$ portion of the black contour in (a), indicating where the mean flow shifts from eastward to westward velocities. (d) Time series of the total eastward volume transport associated with the upper $985-\mathrm{m}$ of the zonal velocities in (a), along $67^{\circ}-41^{\circ} \mathrm{S}$. (e) Time series of the southern and northern portions of the total eastward transport in (d). The value corresponding to their first time step (1920) is indicated, while the y-axis varies in 1-Sverdrup intervals $\left(1 \mathrm{~Sv}=10^{6} \mathrm{~m}^{3} \cdot \mathrm{s}^{-1}\right)$. The gray dashed vertical line indicates the transition from the historical (1920-2005) to the RCP8.5 (2006-2100) period. The correlation coefficient between both detrended, standardized time series is indicated $(-0.74)$. 
(a) Mean $10^{\circ} \mathrm{W}-25^{\circ} \mathrm{E}, 1920-2100$ Zonal Velocities:

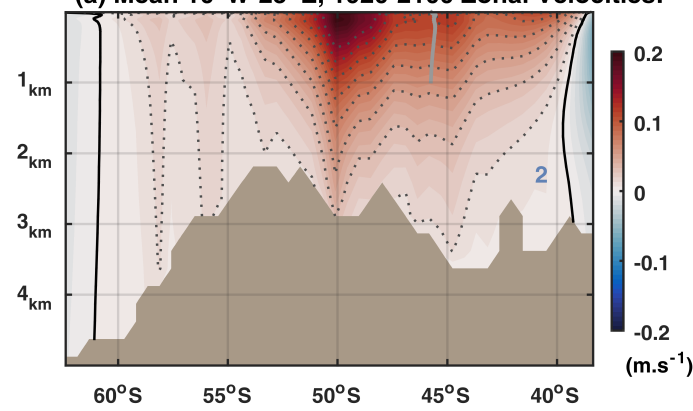

(b) Hovmoller diagram $-10^{\circ} \mathrm{W}-25^{\circ} \mathrm{E}$, upper $985 \mathrm{~m}$ :

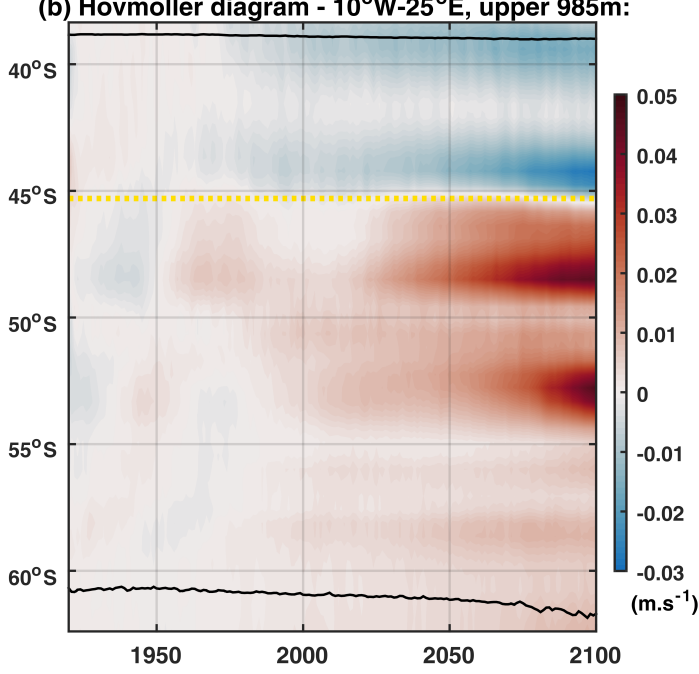

(c) Linear trends of Zonal Velocities [10W-25ㅌ]
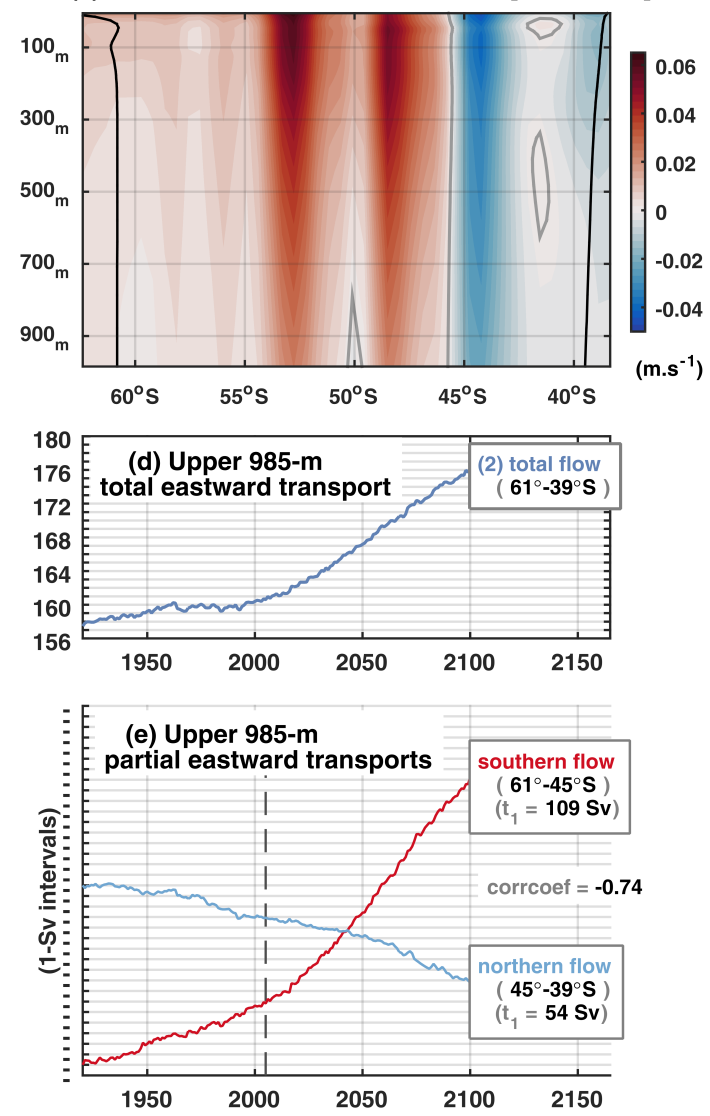

Figure 23 Southern eastward flow through the eastern South Atlantic sector $\left(10^{\circ} \mathrm{W}-25^{\circ} \mathrm{E}\right)$ | Zonal velocities in the southeastern Atlantic sector, averaged over $10^{\circ} \mathrm{W}-25^{\circ} \mathrm{E}$ (box number 2 in Figure 21 a). (a) 1920-2100 mean vertical profile; red denotes positive (eastward) and blue denotes negative (westward) velocities. The black filled contour represents the zero zonal velocity line; and the black dotted contours indicate zonal velocity lines along values of $14 \times 10^{-3}: 2 \times 10^{-2}: 154 \times 10^{-3}$. The gray filled contour in the upper $1-\mathrm{km}$ is reproduced from (c), for reference. (b) Hovmoller diagram (upper 985-m mean) of anomalous zonal velocities (1920-1970 base period); red (blue) indicates positive/eastward (negative/westward) anomalies. The black contour represents the temporal evolution of the upper 985-m, 1920-2100 mean zero zonal velocity line in (a). The yellow dotted line marks the mean latitude of transition between positive and negative anomalies $\left(45.3^{\circ} \mathrm{S}\right)$, in correspondence to the full-depth gray contour in (c), used as a reference to derive the southern and northern volume transports in (e). (c) 1920-2100 total trends of anomalous zonal velocities, corresponding to the upper 985-m layer in (a). The gray filled contour marks the transition from negative (blue) to positive (red) trends. The black filled contour corresponds to the upper $985-\mathrm{m}$ portion of the black contour in (a), indicating where the mean flow shifts from eastward to westward velocities. (d) Time series of the total eastward volume transport associated with the upper $985-\mathrm{m}$ of the zonal velocities in (a), along $61^{\circ}-39^{\circ} \mathrm{S}$. (e) Time series of the southern and northern portions of the total eastward transport in (d). The value corresponding to their first time step (1920) is indicated, while the $y$-axis varies in 1-Sverdrup intervals $\left(1 \mathrm{~Sv}=10^{6} \mathrm{~m}^{3} \cdot \mathrm{s}^{-1}\right)$. The gray dashed vertical line indicates the transition from the historical (1920-2005) to the RCP8.5 (2006-2100) period. The correlation coefficient between both detrended, standardized time series is indicated $(-0.74)$. 
(a) Mean $26^{\circ}-45^{\circ} \mathrm{E}, 1920-2100$ Zonal Velocities:

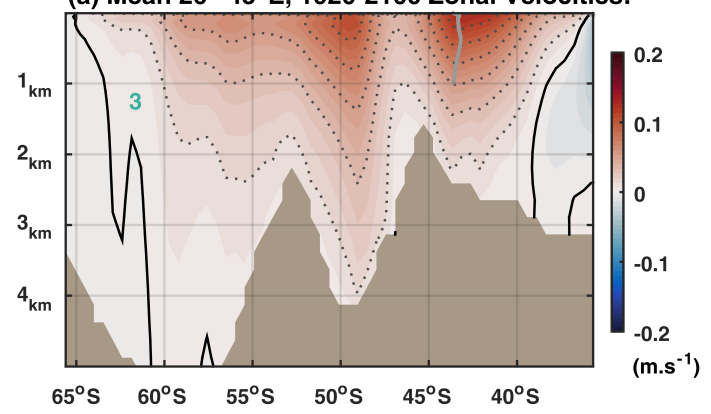

(b) Hovmoller diagram $-26^{\circ}-45^{\circ} \mathrm{E}$, upper $985 \mathrm{~m}$ :

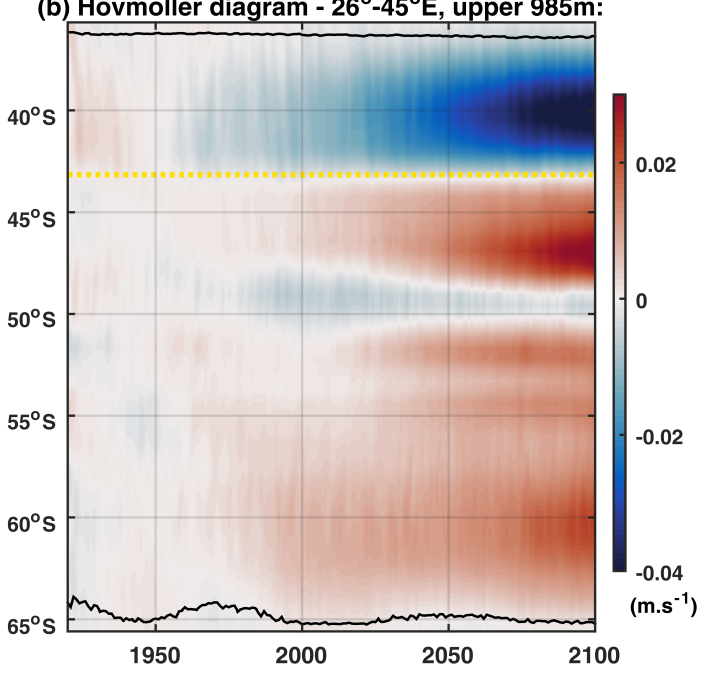

(c) Linear trends of Zonal Velocities [26-.45트
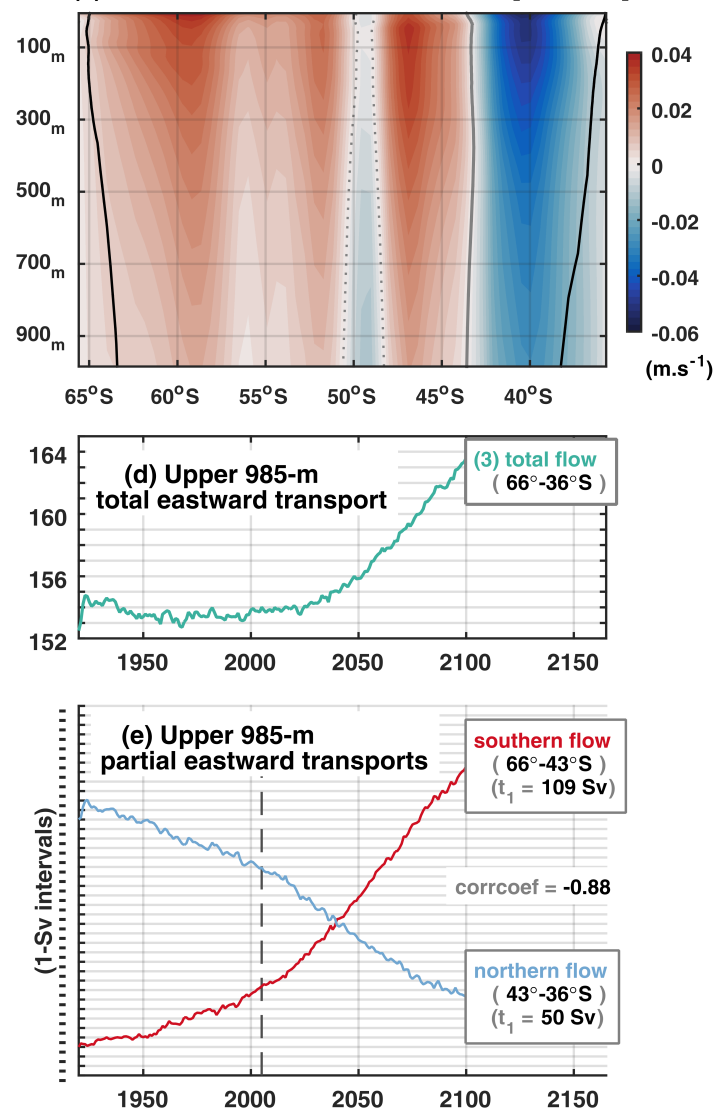

Figure 24 Southern eastward flow entering the Indian Ocean sector $\left(26^{\circ}-45^{\circ} \mathrm{E}\right) \mid$ Zonal velocities of currents leaving the Atlantic towards the Indian basin, averaged over $26^{\circ}-45^{\circ} \mathrm{E}$ (box number 3 in Figure 21a). (a) 1920-2100 mean vertical profile; red denotes positive (eastward) and blue denotes negative (westward) velocities. The black filled contour represents the zero zonal velocity line; and the black dotted contours indicate zonal velocity lines along values of $14 \times 10^{-3}: 2 \times 10^{-2}: 154 \times 10^{-3}$. The gray filled contour in the upper $1-\mathrm{km}$ is reproduced from (c), for reference. (b) Hovmoller diagram (upper 985-m mean) of anomalous zonal velocities (1920-1970 base period); red (blue) indicates positive/eastward (negative/westward) anomalies. The black contour represents the temporal evolution of the upper $985-\mathrm{m}, 1920-2100$ mean zero zonal velocity line in (a). The yellow dotted line marks the mean latitude of transition between positive and negative anomalies $\left(43.2^{\circ} \mathrm{S}\right)$, in correspondence to the full-depth gray contour in (c), used as a reference to derive the southern and northern volume transports in (e). (c) 1920-2100 total trends of anomalous zonal velocities, corresponding to the upper 985-m layer in (a). The gray filled contour marks the transition from negative (blue) to positive (red) trends. The black filled contour corresponds to the upper $985-\mathrm{m}$ portion of the black contour in (a), indicating where the mean flow shifts from eastward to westward velocities. (d) Time series of the total eastward volume transport associated with the upper $985-\mathrm{m}$ of the zonal velocities in (a), along $66^{\circ}-36^{\circ} \mathrm{S}$. (e) Time series of the southern and northern portions of the total eastward transport in (d). The value corresponding to their first time step (1920) is indicated, while the y-axis varies in 1-Sverdrup intervals (1 Sv $=10^{6}$ $\left.\mathrm{m}^{3} . \mathrm{s}^{-1}\right)$. The gray dashed vertical line indicates the transition from the historical (1920-2005) to the RCP8.5 (2006-2100) period. The correlation coefficient between both detrended, standardized time series is indicated $(-0.88)$. 
Analogously, in panels (c) - for the 1920-2100 total trends of the anomalous zonal velocities corresponding to the upper 985-m of panels (a) - the black contours along depth delimit the region where the anomalous zonal velocities correspond to deviations from a climatological eastward flow.

From panels (b) and (c), a pattern of meridional asymmetry in the zonal velocity anomalies emerges. Overall (relative to the meridional extension of each box), while the southern portions of the eastward flow tend to increase in velocities, the northern portions tend to decrease.

The Hovmoller diagrams in panels (b) give a temporal perspective showing the onset of positive/negative anomalies in time, and the vertical profile trends in panels (c) provide a spatial perspective confirming that this pattern is indeed manifesting in the entire upper-ocean layer, above 985-m.

In panels (c), the gray contour separates positive from negative zonal velocity anomalies; this contour is reproduced in panels (a), for reference - indicating where, in the wide climatological eastward cores, it is placed the latitude of transition between the southern portions where eastward velocities tend to strengthen (region to the left of gray contours) and between the northern portions where eastward velocities tend to weaken (region to the right of gray contours).

Although in panels (b) some less diffuse anomalies start to develop from the 1950s on, bolder anomalies usually arise past the early 21 st century, and it is by the mid 21 st century onward that persistent positive and negative anomalous signatures prevail. Positive anomalies (located south of the yellow dotted lines) are usually greater in magnitude over negative anomalies (located north of that), with the exception of the easternmost box (Figure 24b), entering the Indian Ocean sector, where a considerable weakening of the eastward flow at the northern portion is expressed by the vivid blue colors progressively evolving past the 2000s. This can also be seen in Figure 24c for the entire water column above 985-m (negative/blue trends to the right of the gray contour). The different colorbar ranges must be noted when interpreting Figures 22,23 and 24.

Panels (d) display the total eastward transport time series, corresponding to the full meridional extension of the vertical profiles in panels (a) and to the upper $985-\mathrm{m}$. All three total transports undergo an increasing trend, that becomes 
steeper after the 2000s.

The mean latitude of the gray contours dividing positive from negative anomalies in panels (c) is used to define the ranges for deriving partial volume transports associated with the southern and northern portions of the total eastward flow. These opposed portions respectively straddle $53.5^{\circ} \mathrm{S}, 45.3^{\circ} \mathrm{S}$ and $43.2^{\circ} \mathrm{S}$ in Figures 22, 23 and 24. These coordinates are marked by the yellow dotted lines in Figures 22b, c and 23b, 24b.

Finally, the partial southern and northern eastward volume transport time series are shown in panels (e), in which the initial transport value $\left(t_{1}\right)$ associated with each time series is indicated and the $y$-axis varies in 1-Sverdrup (1 Sv $=10^{6} \mathrm{~m}^{3} . \mathrm{s}^{-1}$ ) intervals - so that the approximate magnitude of transport increase/decrease can be visually inferred for the individual time series.

The volume transport time series of the southern and northern parcels (panels (e)) of the mean eastward flows across $100^{\circ}-80^{\circ} \mathrm{W}, 10^{\circ} \mathrm{W}-25^{\circ} \mathrm{E}$ and $26^{\circ}-$ $45^{\circ} \mathrm{E}$ (shown in panels (a)) confirm that even though eastward ocean currents along the Southern Ocean from up- to downstream the South Atlantic sector are generally projected to strengthen (according to panels (d)), this increasing trend is not representative of the full meridional extension comprising these flows. Inspection of the temporal evolution of depth-averaged anomalous zonal velocities along latitude (panels (b)) as well as of 1920-2100 total trends of anomalous zonal velocities on the meridional/depth plane (panels (c)), suggest that only southern counterparts of these eastward flows are accountable for the total transport increase in each of these sections.

When partial transports are derived for the southern/northern parcels, these reveal virtually mirrored time series, with opposed low-frequency signals and long-term trends - where the volume transports associated with northern counterparts actually undergo a sharp decrease. After being detrended and normalized by their respective standard deviation, all northern and southern flow time series exhibit high anticorrelations between them $(r=-0.74$ for the time series in Figures 22e and 23e and $r=-0.88$ for the time series in Figure 24e). This opposing pattern between northern and southern parcels of the eastward flow was also consistently observed at $20^{\circ} \mathrm{W}$ (not shown), an outside region west of box 2 , 
in between boxes 2 and 3 (Figure 21a).

\section{Changes in up- to downstream zonal and meridional volume transports around the Drake Passage and into the South Atlantic}

Even though the flow through the DP is predominantly zonal, meridional components of the velocity field are also present, that are indicative of the upstream origins of the different portions which compose the total volume transport across the DP. Past the DP, the previously narrowed upstream flows finally spread horizontally, partly entering the South Atlantic basin following a northwestward deflection which branches off the ACC to form the MC (Figures 21a, b).

Here, the flows meridionally and zonally outlining the South American coast from the South Pacific to the South Atlantic are investigated (Figure 25).

Just off the southern South American west coast, between $\sim 52^{\circ}-57^{\circ} S$ and $\sim 80^{\circ}-70^{\circ} \mathrm{W}$ in the South Pacific (Figure 21b - 4), it is found a portion of upstream eastward flows that hits the coast and is deflected southward. These waters are expected to be associated with residual flows from the transport along the southern boundary of the SPSG - which end up joining the northern ACC prior to flowing into the DP.

Based on flow continuity principles, a spinned-up SPSG would increase the anticyclonic gyre transport, advecting more waters to the north at the South Pacific eastern boundary and therefore shedding less waters to the east at the southeastern base of the gyre before closing the anticlockwise circulation loop.

The southward volume transport time series along this region is shown in Figure $25 \mathrm{~g}$ - (4) and the vertical profile associated with this time series is shown in Figure $25 \mathrm{a}$ - (4). The latter consists of an individual section within the $52^{\circ}-57^{\circ} \mathrm{S}$ box range, which depicts the western southward and eastern northward flows around South America southernmost tip (the Cape Horn).

The southward transport associated with time series (4) displays a continued decrease, beginning slightly past the 1950s. This decreased transport is in agreement with the recent spin-up of the South Pacific subtropical gyre (Cai, 2006; Roemmich et al., 2007; Schneider et al., 2007; Zhang and Qu, 2015), in that less waters escape from the diffuse eastern boundary of the subtropical gyre to 
leak toward the South American coast - thus being partially deflected eastward and southward into the DP. This signal is also in agreement with the projected weakening of the northern counterpart of the total eastward transport prior the DP at the South Pacific sector of the Southern Ocean (Figure 22).

Except for the southward transport depicted by time series (4), the remaining time series displayed in Figure $\mathbf{2 5} \mathbf{g}$ generally exhibit low-frequency transport variations during the historical period (1920-2005) and pronounced increasing long-term trends along the 21st century (5-11). The rationale behind the choices for deriving these time series and the circulation changes they represent are described hereafter.

The vertical profiles 5 and $\mathbf{6}$ in Figures $\mathbf{2 5} \mathrm{b}, \mathrm{c}$ correspond to two subsequent zonal sections where the DP is closed to the north and south by the adjacent continental barriers (transects shown in Figure 21a - 5, 6). These profiles correspond to the eastward volume transport time series (5) and (6) in Figure 25. $\mathbf{g}$.

The $55^{\circ}-61^{\circ} \mathrm{S}$ box range associated with time series (7) is represented by the vertical profile to the right of the South American continent in 25a - 7, also consisting of an individual section within the respective range depicting the northward flows east of southern South America. This time series (7) represents a meridional component of the total flow crossing the DP (see box 7 in Figure 21b).

Further downstream to the north, box 8 (Figure 21p), associated with vertical profiles 8 (Figures 25 d, e) and time series (8) (Figure 25g), comprises the northward flow that enters the South Atlantic basin at the $\mathrm{MC}$ region, up to its northernmost extension until its confluence with the southward BC. Two exemplary vertical profiles associated with this box/time series are shown in Figures 25d, e in order to demonstrate how the MC core width varies along latitude within this region, mostly in response to the irregular bathymetry along the coast.

Nevertheless, all single time series associated with each individual section within this box range display a very similar low-frequency pattern (not shown), that is properly represented by time series (8) (which consists of an average of singlesection time series from $43^{\circ} \mathrm{S}$ to $54^{\circ} \mathrm{S}$ ). Since northward transport calculations do not account for southward flows that might exist within the respective transect/box range, near the target currents, the fact that box $\mathbf{8}$ is considerably wide and seems 
to partially encompass southward meridional flows does not affect our results or conclusions by any means.
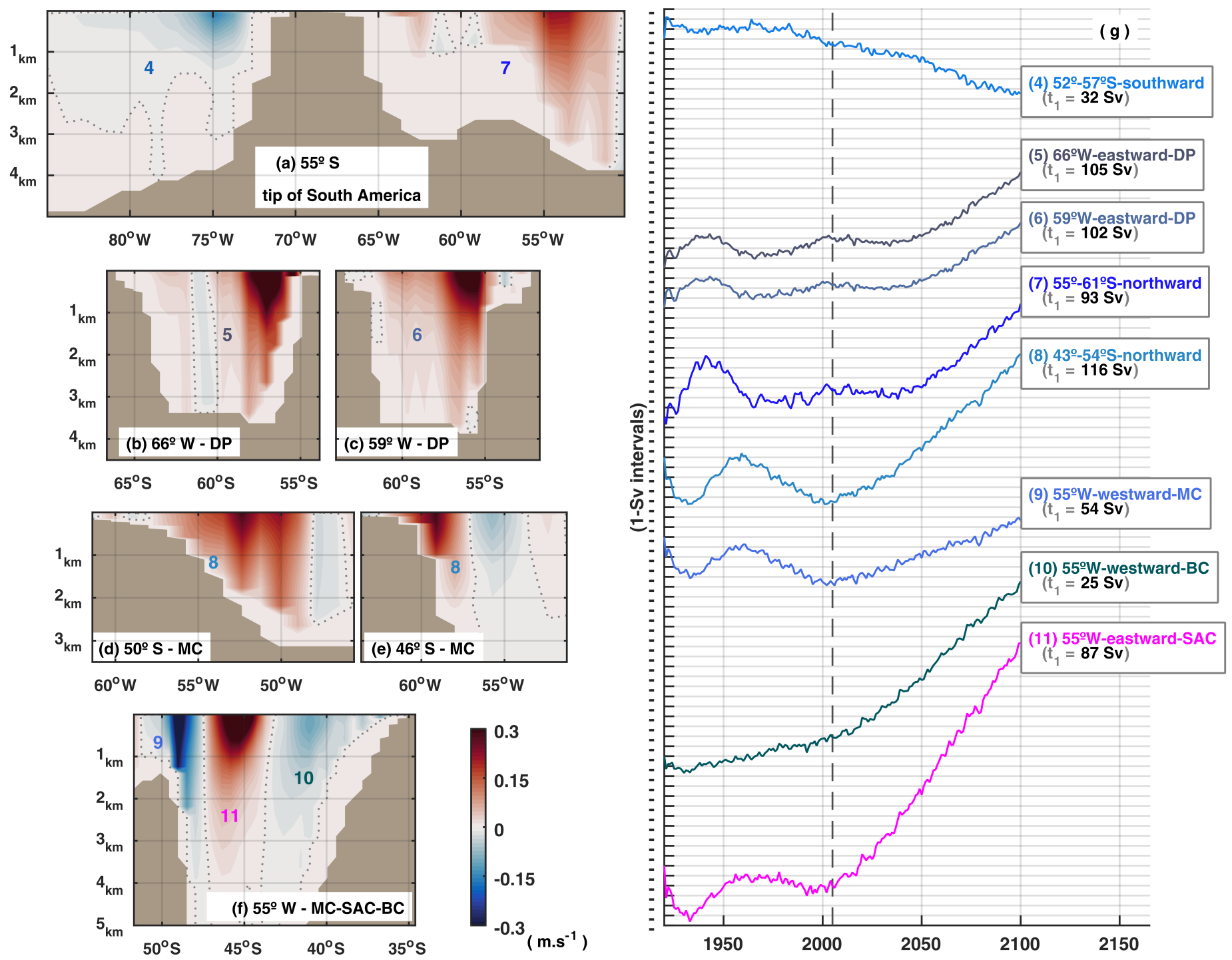

Figure 25 | Vertical profiles and corresponding volume transport time series from the Drake Passage into the South Atlantic | 1920-2100 mean meridional (a, d, e) and zonal (b, c, f) velocities. On Figures 25 and 26, profiles $x / y$-axis ranges are scaled in order to match each other, providing a perspective of relative flow width and depth. The same colorbar scale and contour intervals is also used to give a perspective of relative flow strength and distribution. Numbers indicated within profiles correspond to the numbers indicated on the maps from Figures 21a, b, as well as to the numbers corresponding to each time series (right panel). The volume transport time series correspond to the integration in the upper 985-m layer (slightly above 1-km) across each profile. Time series $\mathbf{4 , 7}$ and $\mathbf{8}$ represent the mean transport along a meridional range, which can be seen on Figures 21a, b. Profiles (a-4,7), (d-8) and (e-8) are contained within the corresponding box ranges of time series 4, 7 and 8 . The value corresponding to the first time step (1920) of each time series is indicated, while the $y$-axis varies in 1-Sverdrup intervals $\left(1 \mathrm{~Sv}=10^{6} \mathrm{~m}^{3} . \mathrm{s}^{-1}\right)$. The gray dashed vertical line indicates the transition from the historical (1920-2005) to the RCP8.5 (2006-2100) period. DP = Drake Passage,$M C=$ Malvinas Current, $S A C=$ South Atlantic Current, $\mathrm{BC}=$ Brazil Current. 
transect at $55^{\circ} \mathrm{W}(21 \mathrm{a}-9-11)$ : (i) encompassing the westward component of the $M C$ into the SAO, to the south (9); (ii) the westward component of the southern end of the BC by delineating the South American coastline, to the north (10); (iii) and the origins of the SAC after the BMC, as a strong eastward core amidst both the $M C$ and the $B C$ (11). Their single vertical profile is illustrated in Figure 25F.

With respect to the low-frequency variations and projected long-term changes expressed by time series 5-11, a virtually common multidecadal oscillation is found throughout 1920-2005 (to the left of the gray dashed line) among them, with the exception of the westward $\mathrm{BC}$ time series across $55^{\circ} \mathrm{W}$ (10): overall, they initially undergo an increase in transport - which peaks around $\sim 1950$, with downstream time series lagging the upstream ones from 5 to 9,11 - followed by a decrease that varies in magnitude for each of the time series.

However, while time series 5,6 and 7 follow a relatively short-lasting decrease and undergo another similar, but smoother, up and down oscillation (before following an ultimate long-term increasing trend that lasts until 2100); time series 8,9 and 11 only go through the course of the first respective oscillation, reaching a minimum precisely by the end of the historical period (2005 - marked with the gray dashed line), and following pronounced increasing transport linear trends thereafter.

This suggests that low-frequency signals are effectively transmitted downstream from the DP (time series 5-7) into the South Atlantic through the MC (time series 8-9) and thereafter to the SAC formation towards the east along the SASG southern boundary. Considering that a whole anticlockwise gyre circulation must be completed in order to connect the initial portion of the SAC at the southern boundary to the final portion of the BC at the end of the SASG western boundary, it is plausible that by getting to the southern BC this 1920-2005 multidecadal signal has been lost. Hypothetically, it is probably dissipated along the downstream southern boundary of the SASG towards the east - since it is no longer present in the AL time series at the SASG southeastern boundary (shown in Figure 26 $\mathrm{h}$ 12 ), by the point the gyre performs a turn around to the north-east.

It is also interesting to note how the aforementioned low-frequency signals are gradually lost along the time series punctuating the downstream flow excursion, 
particularly the transition from time series 5 and 6 (transports through the DP) to time series 8 and 9 (northward and westward MC transports) - accomplished by time series 7 in between both these pairs. More specifically, the second oscillation that is present in time series $5-7$ is suddenly lost, giving place to a steepest decrease towards the end of the historical period, that is promptly followed by the striking long-term increasing trend extending until 2100.

The BC transport time series (10), in turn, follows an increasing trend all the way - that is accelerated past the historical period. Of all time series in Figure 25, the SAC transport (11) displays the highest increase, probably reflecting the build-up of the increasing $\mathrm{MC}$ and $\mathrm{BC}$ contributions.

\subsubsection{Agulhas Leakage, SASG recirculation and AMOC}

\section{Contextualization}

The Indian western boundary AC loops back into the Agulhas Return Current (ARC) by reaching the southern tip of Africa at its eastern coast. In retroflecting to the Indian Ocean, part of the AC waters "leak" into the South Atlantic via the $A L$ in the form of mesoscale eddies, filaments and direct inflow (Durgadoo et al., 2017; Rühs et al., 2019). The AL then embeds into the anticyclonic SASG circulation through the BeC-sSEC system along the northeastern boundary and, by reaching the western boundary, it either follows the northward AMOC upper limb path - effectively providing its "warm water route" contribution - or it recirculates in the SASG along the southward BC. Lagrangian model analysis suggest a nearly equable partition between both these systems - with about $50 \%$ of $\mathrm{AL}$ waters proceeding north in favor of the AMOC and the other $\sim 50 \%$ turning south within the SASG circulation (Rühs et al., 2013).

Here are investigated the pathways along which occur broad westward and subsequent narrow north- and southward spreading of subtropical waters across the SAO basin and along its western boundary. More specifically, the volume transports along the $A L$, the SSEC sub-branches, the poleward $B C$ and the equatorward NBUC. Lastly, the time series of northward transbasin transports and the traditional MOC streamfunction are explored. 


\section{Changes in up- to downstream volume transports from the Agulhas}

\section{Leakage to the SASG closure and the AMOC upper limb fate}

The up- to downstream time series sequentially shown in Figure $26 \mathrm{~h}$ clearly illustrate a long-term trend reversal across their associated upper-ocean circulation pathways.

Despite the southward AC flow seems to weaken according to the anomalous trends presented in Figure $\mathbf{2 1} \mathbf{d}$, the westward transport across $12^{\circ} \mathrm{E}$ into the South Atlantic shows a long-term increase (Figure 26h - 12 - associated with vertical profile 12 in Figure 26a). Downstream to the west, the southern sSEC transport presents an increase of similar magnitude (time series 13 - associated with the parcel 13 of the sSEC vertical profile in Figure 26b). And further downstream, the final portion of the southward $\mathrm{BC}$ at $37^{\circ} \mathrm{S}$ (which is meridionally aligned with the arriving westward flow of the southern SSEC) presents an increased transport of even greater magnitude (time series 14 - associated with vertical profile 14 in Figure 26c).

The BC transport upstream of that, at $28^{\circ} \mathrm{S}$, increases as well (time series 15 - associated with vertical profile 15 in Figure 26d). The BC transport is considered weak near its site of origin and it is increased from north to south as the BC core gets deeper (e.g. Schmid and Majumder, 2018). The CESM-LE results simulate a considerably weak $\mathrm{BC}$ at $28^{\circ} \mathrm{S}$, yet, it displays a noticeable increasing long-term trend.

A possible explanation derives from the fact that even though the central and northern portions of the SSEC (time series 16 and 17 - associated with parcels 16 and 17 of the SSEC vertical profile in Figure $26 \mathrm{~b}$ ) respectively show no trend at all (16) or even undergo a steady decrease in transport (17), part of their flow reaching the western boundary still turn southward to feed the $\mathrm{BC}$ - gradually increasing its transport along southern latitudes. Therefore, even though the BC at $28^{\circ} S$ is located north of the latitudinal band that receives the southern SSEC increased flow, its transport undergoes an increase as well, in response to the build-up of the central and northern SSEC transport contributions. 

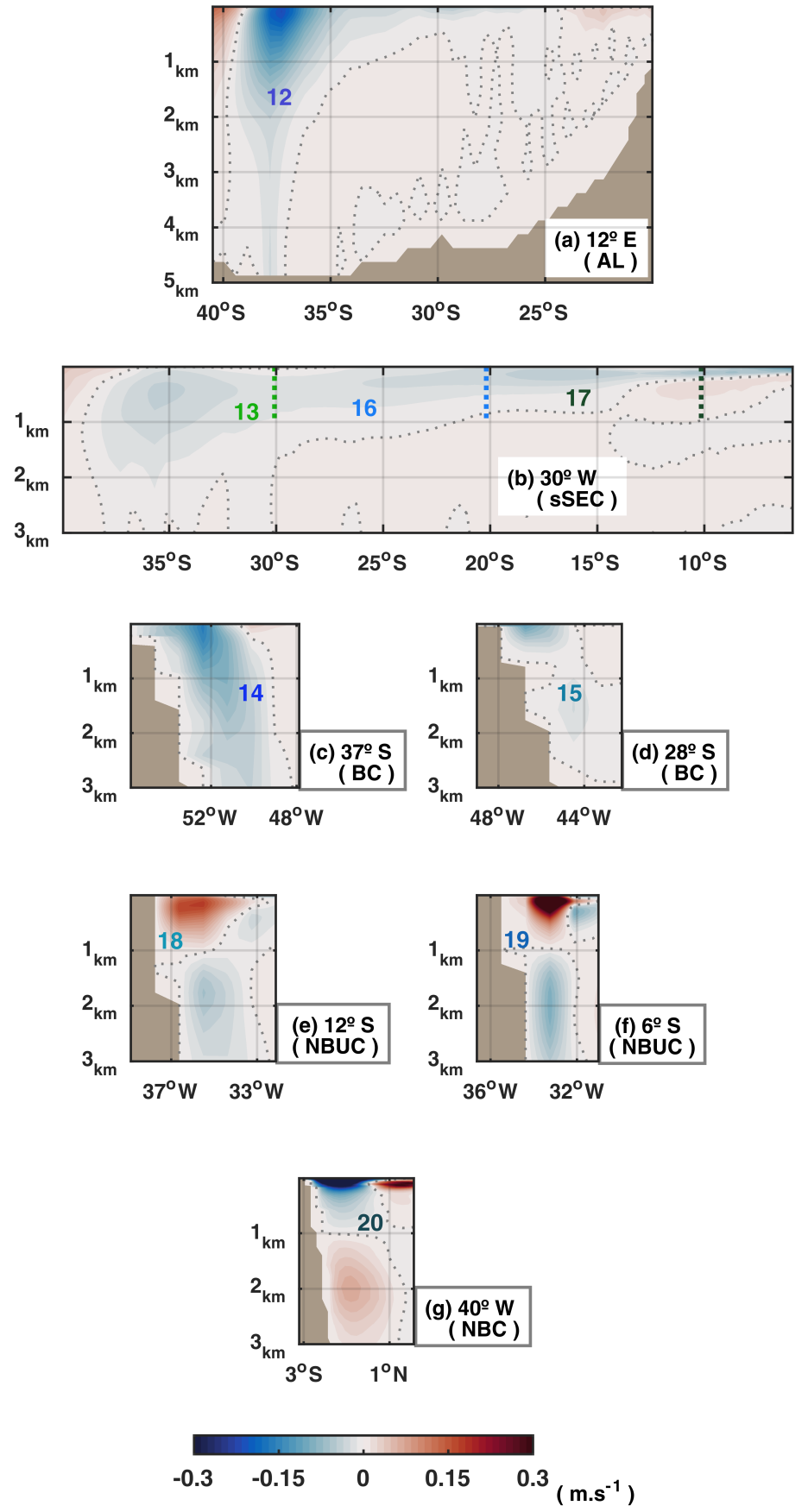

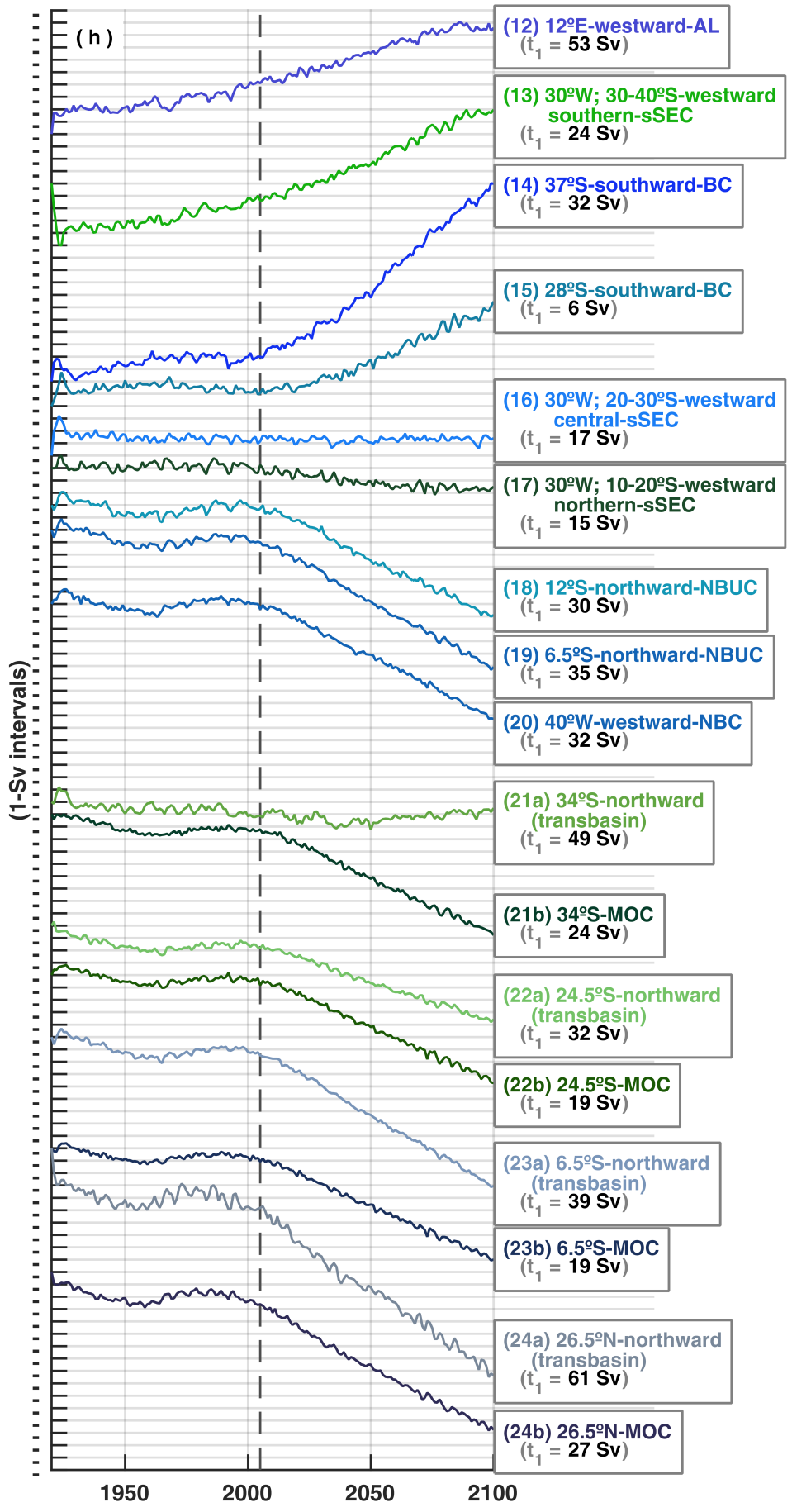

Figure 26 | Vertical profiles and corresponding volume transport time series from the Agulhas Leakage towards the South Atlantic western boundary and downstream beyond 1920-2100 mean meridional (c, d, e, f) and zonal (a, b, g) velocities. On Figures 25 and 26 , profiles $\mathrm{x} / \mathrm{y}$-axis ranges are scaled in order to match each other, providing a perspective of relative flow width and depth. The same colorbar scale and contour intervals is also used to give a perspective of relative flow strength and distribution. Numbers indicated within profiles correspond to the numbers indicated on the maps from Figures $21 \mathrm{a}, \mathrm{b}$, as well as to the numbers corresponding to each time series (right panel). The volume transport time series correspond to the integration in the upper $985-\mathrm{m}$ layer (slightly above $1-\mathrm{km}$ ) across each profile, except for the MOC time series $(21 \mathrm{~b}$, $22 \mathrm{~b}, 23 \mathrm{~b}, 24 \mathrm{~b}$ ), which are vertically integrated up to the last depth of northward zonally integrated meridional velocities. The value corresponding to the first time step (1920) of each time series is indicated, while the $y$-axis varies in 1-Sverdrup intervals $\left(1 \mathrm{~Sv}=10^{6} \mathrm{~m}^{3} \cdot \mathrm{s}^{-1}\right)$. The vertical profiles corresponding to time series 21-24 are shown in Figure 27. The gray dashed vertical line indicates the transition from the historical (1920-2005) to the RCP8.5 (2006-2100) period. AL = Agulhas Leakage, sSEC = southern branch of the South Equatorial Current, BC = Brazil Current, NBUC = North Brazil Undercurrent, NBC $=$ North Brazil Current, $\mathrm{MOC}=$ Meridional Overturning Circulation. 
In fact, the northward NBUC transports at $12^{\circ} \mathrm{S}$ and $6.5^{\circ} \mathrm{S}$ undergo a substantial decrease past the historical period (time series 18 and 19 - associated with vertical profiles 18 and 19 in Figures 26e, f), just as the downstream westward NBC transport at $40^{\circ} \mathrm{W}$ (time series 20 - associated with vertical profile 20 in Figure $\mathbf{2 6 g}$ ). This supports the idea that at least the central sSEC flow into the western boundary (which was previously hypothesized to be the main branch that actually bifurcates) is being preferably directed southward to feed the BC, instead of heading northward with the NBUC.

Time series 21a-24a refer (exclusively) to northward volume transports across east-to-west sections along the Atlantic basin, i.e., northward transbasin transports. Analogously, time series $21 \mathrm{~b}-24 \mathrm{~b}$ represent the MOC streamfunction for the same meridional sections - at $34^{\circ} \mathrm{S}, 24.5^{\circ} \mathrm{S}, 6.5^{\circ} \mathrm{S}$ and $26.5^{\circ} \mathrm{N}$.

The MOC streamfunction calculation accounts for both northward and southward meridional flows, and integrates the velocities vertically up to the last depth of zonally integrated northward velocities in each time step. Therefore, time series 21a-24a and 21b-24b differ principally in that the former accounts only for waters being transported northward - without discounting southward returning flows, while the latter accounts for both.

In observing time series 21-24, it is clear that these all show decreasing transport trends, with the exception of $21 \mathrm{a}$. This means that at $34^{\circ} \mathrm{S}$, the decrease in the MOC streamfunction (21b) is mostly due to increased southward flows instead of decreased northward transports - since time series 21a shows no decreasing trend. This is in line with the increased southward BC transports (14, 15) that consequently act to decrease the magnitude of the total MOC transport.

The northward transport at $34^{\circ} \mathrm{S}$, reflected by time series $21 \mathrm{a}$, consists of the only transbasin northward transport that does not follow a decrease with time probably because, at this latitude, the northward component of the strengthened AL-BeC-sSEC system acts to sustain these transbasin northward transport values - not allowing a transport decrease. However, the waters flowing northward across $34^{\circ} \mathrm{S}$ do not reach any further, after all - at least not up to $24.5^{\circ} \mathrm{S}$ (see time series 22a). That is because these are mostly recirculating within the SASG southwestern core, promptly feeding the southern (and to a lesser degree, the central) SSEC 
prior turning poleward and contributing to strengthen the $\mathrm{BC}$ flow.

Figures 27a-d show the consecutive transbasin vertical profiles corresponding to time series 21-24 and Figures 27e-h present Hovmoller diagrams of the zonally integrated meridional velocity anomalies (1920-1970 base period) across transbasin profiles.
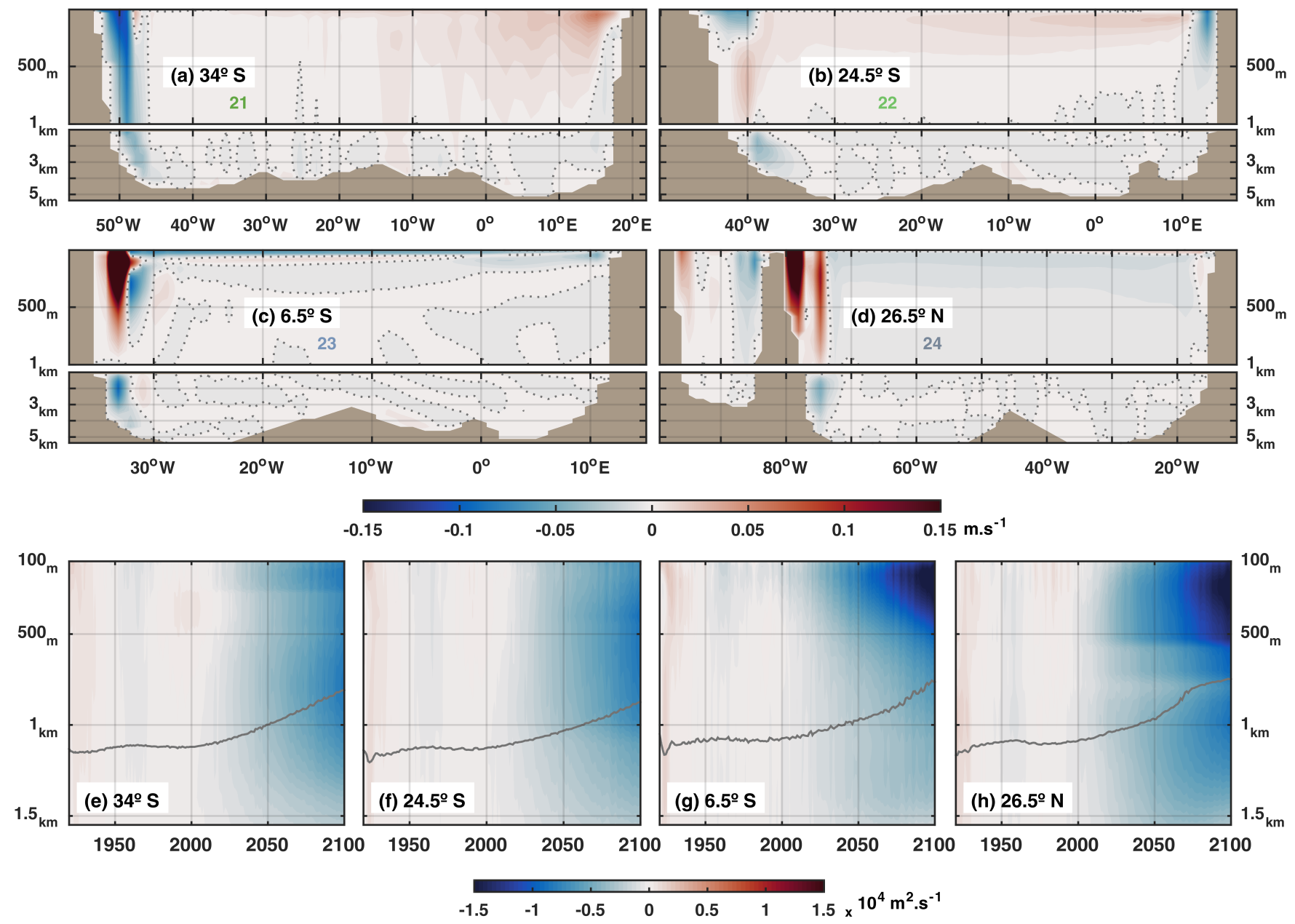

Figure 27 | Transbasin meridional velocity profiles and the evolution of their zonally integrated anomalies $\mid(\mathbf{a})$. (b, c, d) $1920-2100$ mean meridional velocities at $34^{\circ} \mathrm{S}, 24.5^{\circ} \mathrm{S}, 6.5^{\circ} \mathrm{S}$ and $26.5^{\circ} \mathrm{S}$, respectively. (e, $\left.\mathbf{f}, \mathbf{g}, \mathbf{h}\right)$ Hovmoller diagrams of the upper $\sim 1.5-\mathrm{km}$ zonally integrated meridional velocity anomalies in each section. Blue (red) denotes negative/southward (positive/northward) anomalies. The gray filled contour marks the 1920-2100 evolution of the zero meridional velocity line that delimits the lower limit of the upper limb of the Atlantic Meridional Overturning Circulation. The upper 100-m of Hovmoller diagrams are not shown since they reflect near-surface dynamics within the Ekman layer, which are out of the scope of this study.

The gray contours in Figures 27e-h indicate the evolution of the mean zero meridional velocity line, which delimits the lower limit of the AMOC upper limb. Considering the raw (not anomalous) velocities, the MOC streamfunction time series for each profile is then obtained one step ahead, by vertically integrating 
these meridional velocities in each time step up to the depth where the zero meridional velocity line lies.

A common result to all profiles is that the AMOC upper limb is getting shallower with time - shrinking to depths shallower than 1-km past the year of 2050. Regarding the temporal propagation of anomalies along depth, while the $34^{\circ} \mathrm{S}$ and $24.5^{\circ} \mathrm{S}$ sections display widespread negative anomalies in the whole water column, sections $6.5^{\circ} \mathrm{S}$ and $26.5^{\circ} \mathrm{N}$ exhibit anomalous decreasing flows mostly concentrated above the 500-m layer.

This can also be interpreted according to the SASG circulation change pattern revealed by the transport time series in Figure 26. up to $24.5^{\circ} \mathrm{S}$ (albeit substantially less than at $34^{\circ} \mathrm{S}$ ), the northward transports are still sustained by the BeC-sSEC system at the eastern boundary of the SASG (note the basin-scale predominant northward velocities in the climatological vertical profile of Figure 27b); at $6.5^{\circ} \mathrm{S}$ and $26.5^{\circ} \mathrm{N}$, basin interior climatological transports far from the western boundary are predominantly southward-oriented (Figures 27c, d) - which means that the upper 500-m striking anomalies in Figures 27. $\mathbf{~} \mathbf{h}$ are in majority associated with decreased NBUC and Gulf Stream (GS) transports off the western boundaries at $6.5^{\circ} \mathrm{S}$ and $26.5^{\circ} \mathrm{N}$, respectively.

In sum, the decreasing trends of the MOC streamfunction time series $(21 b-24 b)$ are in agreement with the currently observed/simulated and projected AMOC slowdown under anthropogenic warming since the industrial revolution based on the modern scientific literature referenced in Section 5.1. Moreover, the CESM-LE projects an AMOC slowdown with meridional coherence across the Atlantic basin, from $34^{\circ} \mathrm{S}$ to $26.5^{\circ} \mathrm{N}$. While northward transports at the eastern boundary of the SASG are sustained up to $34^{\circ} \mathrm{S}$, these signals are not transmitted to lower latitudes; instead, they are destined to recirculate in the SASG - turning back to higher latitudes with the western boundary BC.

\section{SASG structural changes}

Figure 28a shows the upper $\sim 2-k m$ 1920-2100 climatological zonal velocities vertical profile, averaged over $30^{\circ}-10^{\circ} \mathrm{W}$ at the meridional center of the SASG, in the middle of the South Atlantic basin. The westward SSEC is illustrated 
in blue background colors to the north, and the eastward SAC-ACC flows are represented by red background colors to the south. Black contours are the zero meridional velocity line, whose temporal excursion along 1920-2100 is denoted by the overlapping gray contours and 1920-1970 / 2080-2100 climatological means are represented by blue and red dotted contours, respectively - revealing a southward displacement of the SSEC system forming the northern SASG boundary.

In Figure 28b a Hovmoller diagram for the averaged upper 985-m raw zonal velocities is displayed (note the y-axis varying in time, instead of depth as in 28a). Whereas, the analogous Hovmoller diagram in Figure 28c concerns the anomalous zonal velocities (1920-1970 base period). The temporal evolution of the upper $985-\mathrm{m}$ mean zero zonal velocity line is represented by the gray contours in Figure 28b, which as also reproduced in Figure 28c, for reference.

The 1920-2100 evolution of the upper 985-m zonal velocity field (Figure 28b) mainly reveals an increasing trend of the eastward flow centered at $\sim 45^{\circ} S$ as well as of the westward SSEC flow roughly between $30^{\circ}-40^{\circ} \mathrm{S}$. This is the portion here defined as the southern SSEC, shown at 30 W (Figure 26b), whose volume transport across this section was projected to increase (time series 13).

In turn, the increased eastward transport centered at $\sim 45^{\circ} \mathrm{S}$ is associated with the strengthened SAC (time series 11 - Figure 25, and see also the positive trends along $45^{\circ} \mathrm{S}$ on Figure $\mathbf{2 1} \mathrm{d}$ ). The meridional propagation of anomalies (Figure 28c), however, show the onset of developing anomalies, mainly after the year of 2000 , revealing three separated cores of eastward intensification - at $50^{\circ} \mathrm{S}$ and $\sim 58^{\circ} \mathrm{S}$, in addition to the one at $\sim 45^{\circ} \mathrm{S}$.

But, more interestingly, it also corroborates the weakening of the northern counterpart of the mean eastward flow between $30^{\circ}-10^{\circ} \mathrm{W}$, complementing the results shown in Figures 22,23 and 24.

More specifically, beyond negative anomalies developing north of the gray contour (i.e., to the right) - which corroborate the southern SSEC strengthening -, negative anomalies also emerge south of the gray contour (i.e., to the left) revealing a weakening of the eastward SAC at its northern portion, just north of where its greater strengthening occurs (centered at $\sim 45^{\circ} \mathrm{S}$ ). 

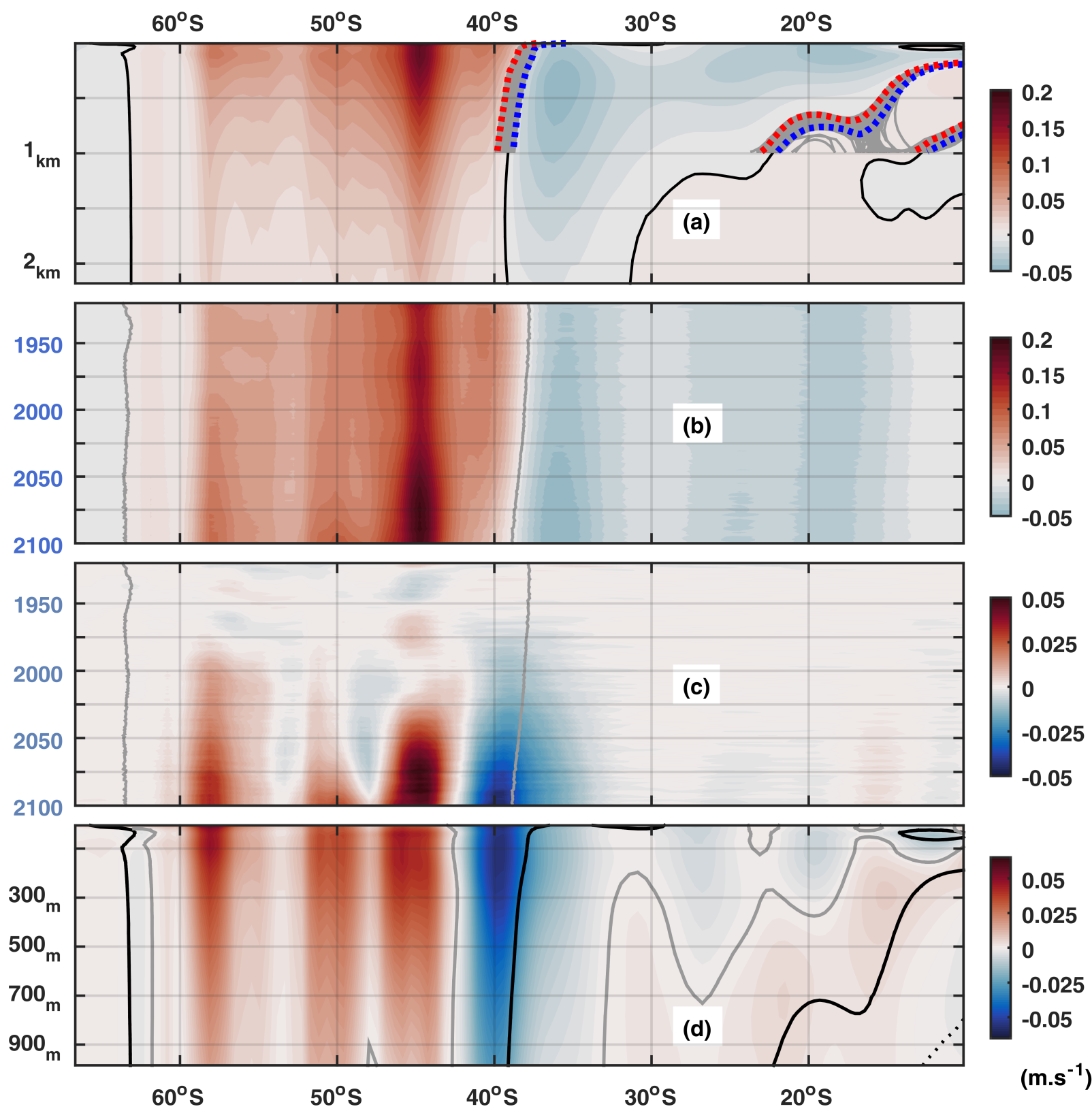

Figure 28 SASG-core $\left(30^{\circ}-10^{\circ} \mathrm{W}\right)$ structural changes | Mean $30^{\circ}-10^{\circ} \mathrm{W}$ climatological (19202100 ) and anomalous (1920-1970 base period) zonal velocities $\left(\mathrm{m} \cdot \mathrm{s}^{-1}\right)$ centered at the South Atlantic subtropical gyre (SASG): (a) Climatological vertical profile, depicting the westward flow of the consecutive SSEC branches at the northern limb of the subtropical gyre (blue) and the eastward SAC flow at the southern limb of the subtropical gyre, merged with the ACC flow in the Southern Ocean (red). Black contours mark the climatological zero zonal velocity line, whose upper 985-m, 1920-2100 annual evolution is represented by gray contours (filling the whole range of their southward migration since 1920), delimited by the 1920-1970 (blue dotted contour) and 2080-2100 (red dotted contour) climatologies. (b, c) Hovmoller diagram of averaged upper 985-m raw (b) and anomalous (c) zonal velocities; red (blue) denotes positive/eastward (negative/westward) raw velocity values (b) / anomalous velocity values (c); the gray thick line in (b) (also reproduced in (c), for reference) marks the temporal evolution of the upper 985-m-mean zero zonal velocity line; (d) 1920-2100 total trends of anomalous zonal velocities corresponding to the upper 985-m in (a). Red (blue) denotes positive (negative) trends, i.e., regions where eastward (westward) anomalies tend to increase; the gray filled contour separates positive from negative trends; the black thick contour corresponds to the upper 985-m climatological zero zonal velocity line in (a), for reference. 
Here, the strengthening of the westward southern SSEC, just north of the zero meridional velocity line, seems to be acting to damp the northern portion of the eastward SAC, which is placed just south of the zero meridional velocity line.

Finally, the 1920-2100 trends of the zonal velocity anomalies along the vertical profile corresponding to the upper 985-m of Figure 28 a is shown in Figure 28d (note the $y$-axis shifts to depth intervals again). Gray contours separate positive from negative anomalies, while black contours are reproduced from Figure 28a, for reference.

Figure 28d provides a spatial perspective of the projected changes for the meridional-depth plane - ultimately confirming that the depth-averaged change patterns shown in Figures 28 b, c apply to the entire vertical layer they represent - homogeneously up to $\sim 33^{\circ} S$, and non-homogeneously from there to the north. The pattern displayed between $\sim 33^{\circ}-\sim 10^{\circ} S$ precisely reflects the strengthening (weakening) of the bowl-shaped southern (northern) portion of the SSEC at the northern boundary of the SASG.

This clarifies why the vertically integrated volume transports of the southern, central and northern SSEC display increasing, nearly-zero and decreasing long-term trends, respectively. Reminding that these transport calculations account only for westward (negative) velocities, which are found in between both black thick contours north of $40^{\circ} \mathrm{S}$ (in Figure 28d), it is possible to see that the region between $\sim 40^{\circ}-30^{\circ} S$ (range which defines the southern SSEC) encompasses mostly negative (i.e., westward-strengthening) anomalies; the region between $\sim$ $30^{\circ}-20^{\circ} S$ (range which defines the central SSEC) encompasses both negative and positive anomalies in a nearly-equable proportion (suggesting they cancel each other producing no westward strengthening nor weakening); and the region between $\sim 20^{\circ}-10^{\circ} S$ (range which defines the northern SSEC) encompasses mostly positive (i.e., westward-weakening) anomalies - thus reflecting the trends expressed by the time series of the southern, central and northern sSEC portions (Figure 26h - 13, 16, 17).

The displacement of zero meridional velocity line between 1920-1970 / 2080-2100 shown in Figure 28a also suggests the subtropical gyre is getting shallower at the northern boundary (between $30^{\circ}-10^{\circ} \mathrm{W}$ ), besides shifting to the 
south - just as the basin-integrated AMOC upper limb is getting shallower as well, at $-34^{\circ} \mathrm{S}, 24.5^{\circ} \mathrm{S}, 6.5^{\circ} \mathrm{S}$ and $26.5^{\circ} \mathrm{N}$, according to Figures $27 \mathrm{e}-\mathbf{h}$.

Figure 29 demonstrates a remarkable southward displacement of the southern SASG boundary at the western portion, between $\sim 50^{\circ}-10^{\circ} \mathrm{W}$ - seen through the zonal-meridional plane (Figure 29a) and through the meridional-depth plane (Figures $29 \mathrm{~b}-\mathrm{e}$ ), and also indicated by the time series of the latitude of the zero zonal velocity line derived for the $100-\mathrm{m}$ depth level, at $40^{\circ} \mathrm{W}, 30^{\circ} \mathrm{W}, 20^{\circ} \mathrm{W}$ and $5^{\circ} \mathrm{W}$ (Figure 29f).
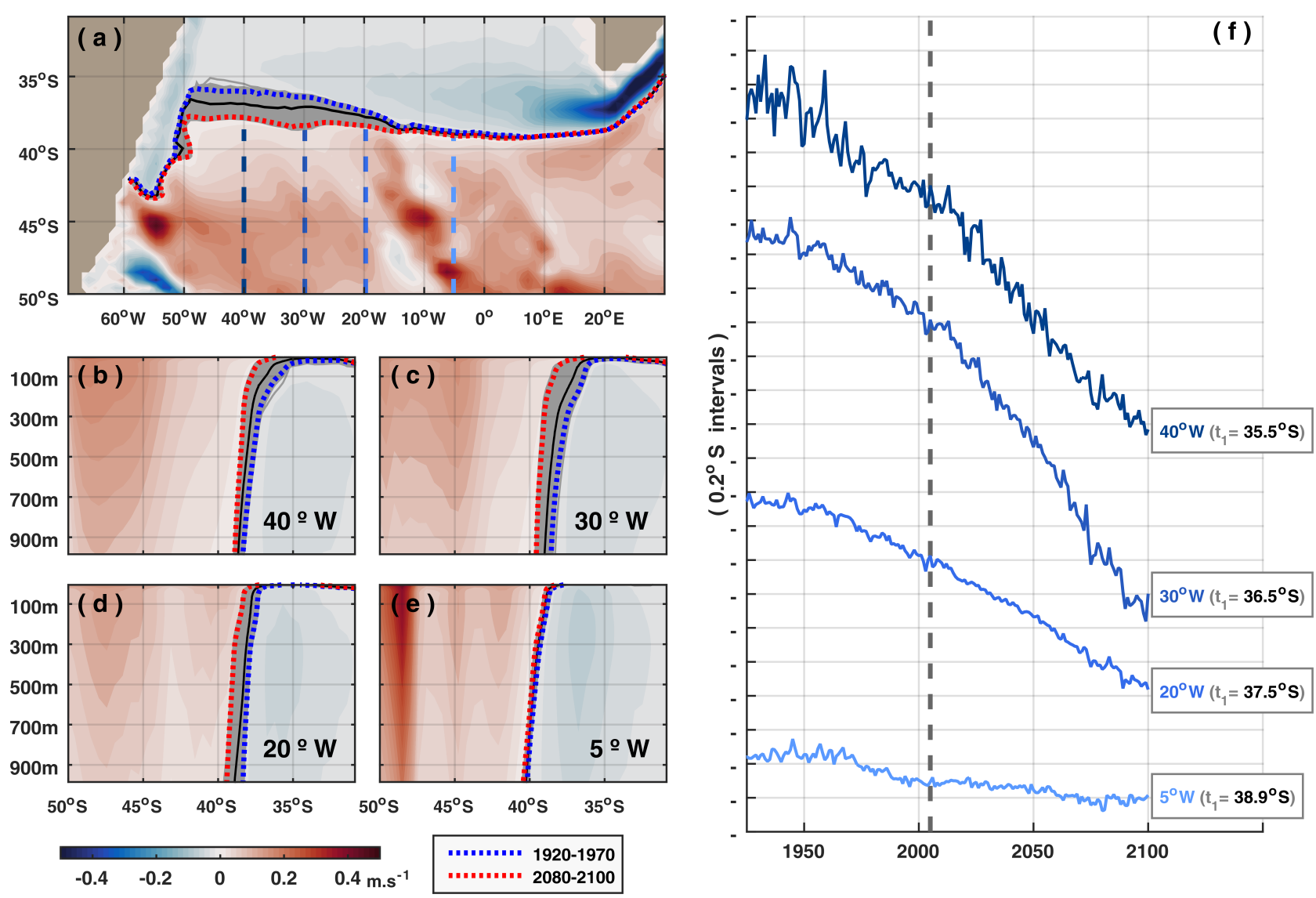

Figure 29 | Poleward shift of the southwestern SASG boundary | (a-e) 1920-2100 mean zonal velocities (background colors) - at the zonal-meridional plane for the upper 985-m average (a) and along depth at $40^{\circ} \mathrm{W}, 30^{\circ} \mathrm{W}, 20^{\circ} \mathrm{W}$ and $5^{\circ} \mathrm{W}(\mathbf{b}, \mathbf{c}, \mathbf{d}$ and e, respectively - marked by the vertical dashed lines in (a)), showing the mean zero zonal velocity contour for: 1920-2100 (black filled contour), 1920-1970 (blue dotted contour) and 2080-2100 (red dotted contour). (f - right panel) Time series of the zero zonal velocity line latitude at $40^{\circ} \mathrm{W}, 30^{\circ} \mathrm{W}, 20^{\circ} \mathrm{W}$ and $5^{\circ} \mathrm{W}$; at $100-\mathrm{m}$ depth. The value corresponding to the first time step (1920) of each time series is indicated, while the $y$-axis varies in $0.2^{\circ}$ latitude (increasing from south to north). The gray dashed vertical line indicates the transition from the historical (1920-2005) to the RCP8.5 (2006-2100) period.

It is shown that this southward displacement is restricted to the region west of $5^{\circ} \mathrm{W}$, at least. These results are also corroborated and complemented by 
the southward shift shown in Figure $28 \mathrm{a}$ for the $30^{\circ}-10^{\circ} \mathrm{W}$ average, as described above.

In addition to the southward migration of the southwestern SASG boundary inferred from the zero zonal velocity line in Figure 29, the northwestern SASG boundary migrates to the north, instead - according to the zero meridional velocity line which defines the SBL (Figure 30).
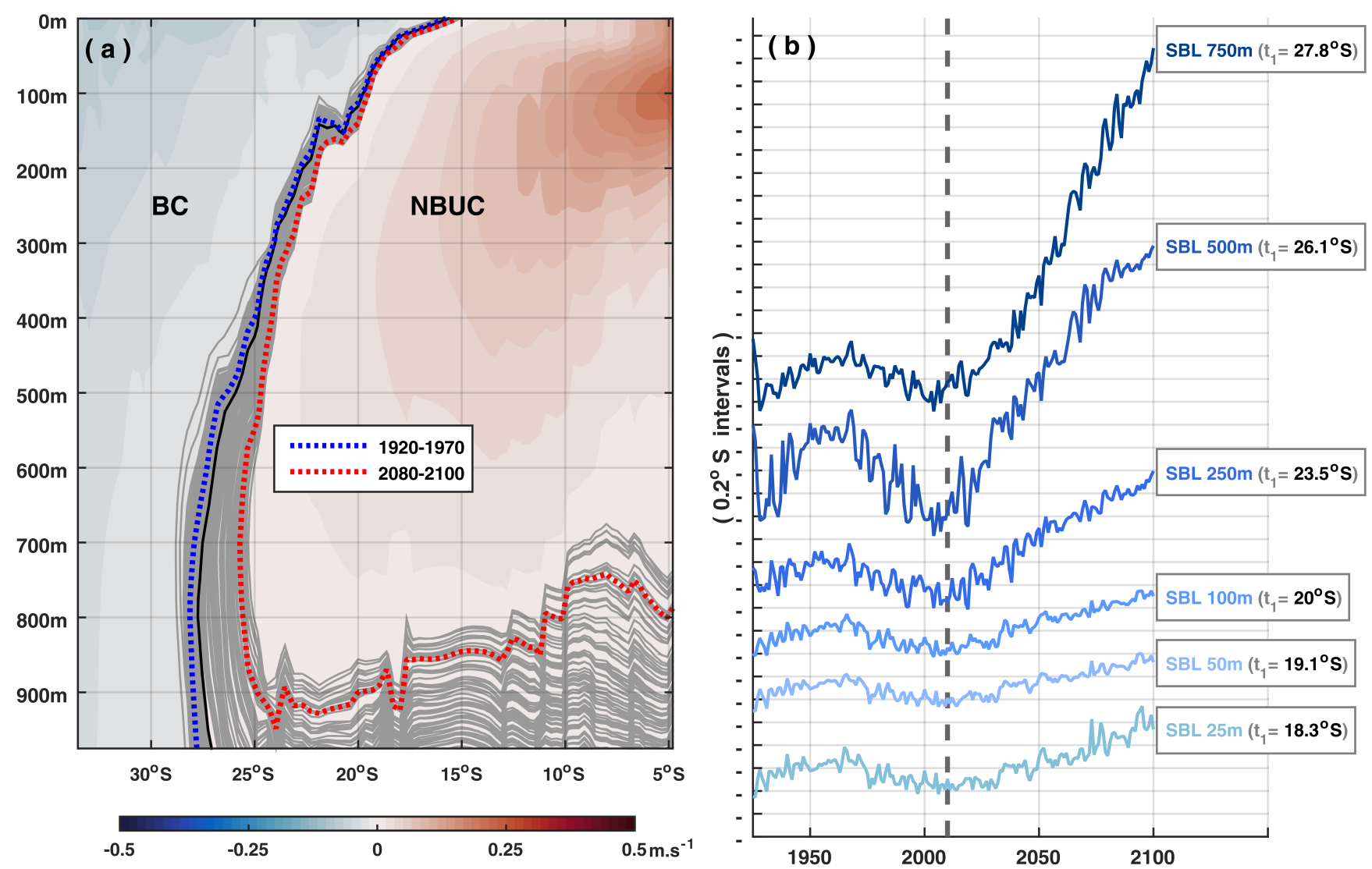

Figure 30 | Equatorward shift of the SSEC bifurcation latitude at the northwestern boundary of the SASG | (a) 1920-2100 mean meridional velocities averaged over the western boundary layer ( $i 4^{\circ}$ from the South American coast). Positive (negative) values indicate northward (southward) flows associated with the NBUC (BC). The bifurcation of SSEC is represented by the zero meridional velocity line, shown for the mean periods of 1920-2100 (black filled contour), 1920-1970 (blue dotted contour) and 2080-2100 (red dotted contour). (b) Time series of the sSEC bifurcation latitude at different depths. The value corresponding to the first time step (1920) of each time series is indicated, while the $y$-axis varies in $0.2^{\circ}$ latitude (increasing from south to north). The gray dashed vertical line indicates the transition from the historical (1920-2005) to the RCP8.5 (2006-2100) period.

Only in this case, this meridional migration does not take place homogeneously along depth - the shift is larger in deeper layers (as expressed by the time series of the latitude of the zero meridional velocity line at consecutive depths from $25 \mathrm{~m}$ to $750 \mathrm{~m}$ - Figure $30 \mathrm{~b}$ ). 
The northward migration of the SBL can be understood in terms of changes in western boundary current transports. The increased southward BC and decreased northward NBUC transports act to push the bifurcation latitude to the north, i.e. the SBL is directed against the flow that strengthens (Rodrigues et al., 2007; Marcello et al., 2018, 2019).

The shallowing of the AMOC upper limb (Figures 27e-h) is also illustrated in Figure 30a by the blue/red dotted (1920-1970 / 2080-2100 means) and gray filled contours (1920-2100 evolution), representing the displacements of the zero meridional velocity line at the bifurcation region and at the lower base of the northward western boundary current - the NBUC - which is the main conduit for the AMOC upper limb north of the SASG.

An EOF analysis has been performed on the detrended zonal velocity anomalies averaged in the upper 985-m (Figure 31a, b). Detrending the data prior to performing the EOF supposedly removes the anthropogenically-forced signal from the time series, leaving the signature of potential modes of internal climate variability.

Interestingly, this leading EOF pattern - which explains $75.8 \%$ of the total variance - is nearly identical to that of the leading EOF of non-detrended zonal velocity anomalies (Figure 38 in Appendix I) - which in turn explains $94.9 \%$ of variance - except that the amplitude (or principal component, PC) time series related to the latter displays a linearly increasing trend instead of a trend reversal as in the $\mathrm{PC}$ related to the detrended EOF.

Here, the detrended EOF demonstrates that during the historical period (1920-2005), predominant anomalies that theoretically concern natural modes of internal variability pointed to a spatial pattern of opposite phase in relation to that of the RCP8.5 period (2005-2100). Whilst, in the non-detrended EOF the phase related to the RCP8.5 period prevails along most of the entire period - more specifically, from the 1950s on - being that, before the 1950s, the amplitude time series varied steadily close to zero. The timing of the phase shift in the detrended EOF corresponds to a period in the non-detrended EOF when the linear increasing trend is accelerated. 

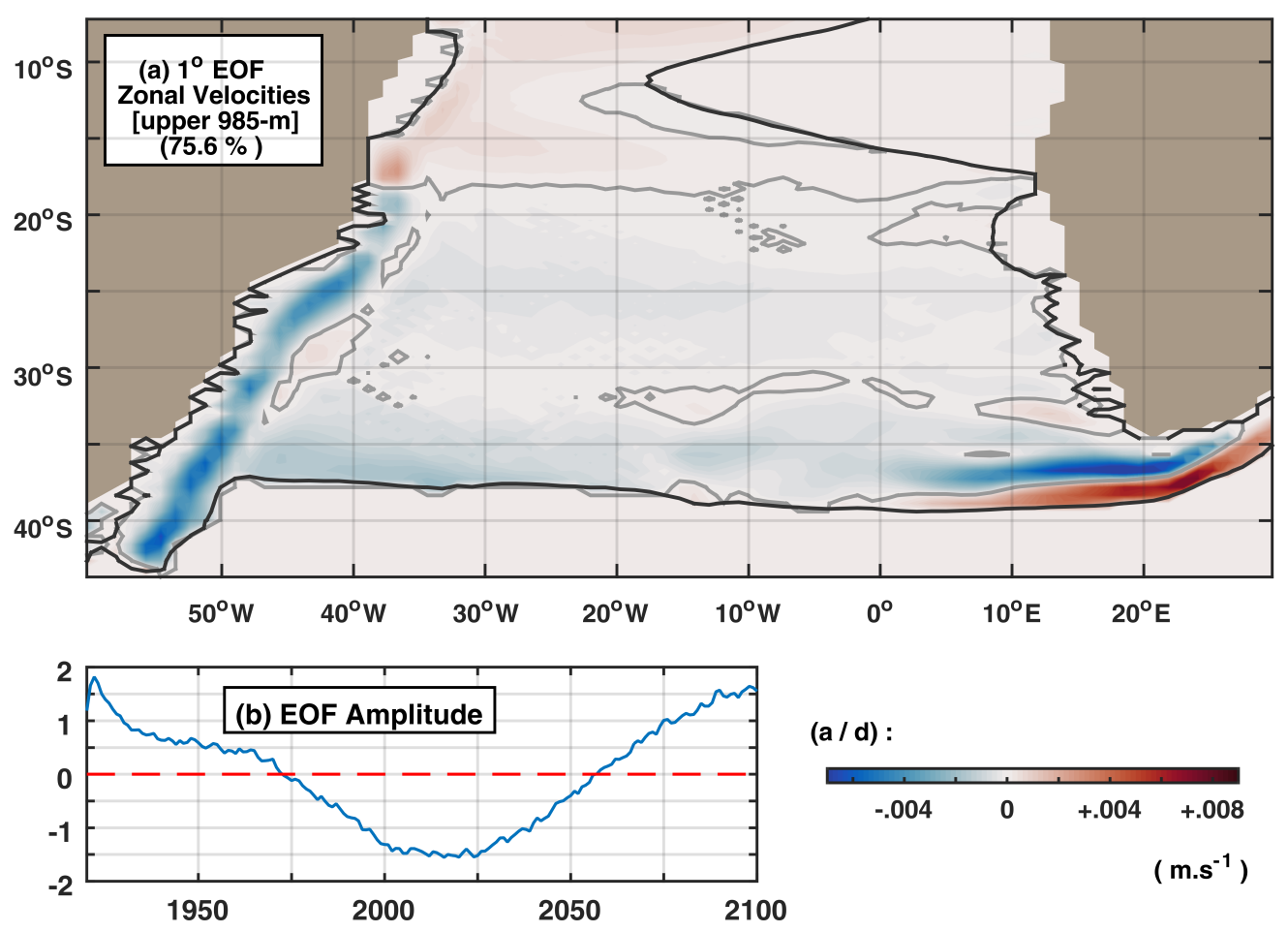

(a/d) :
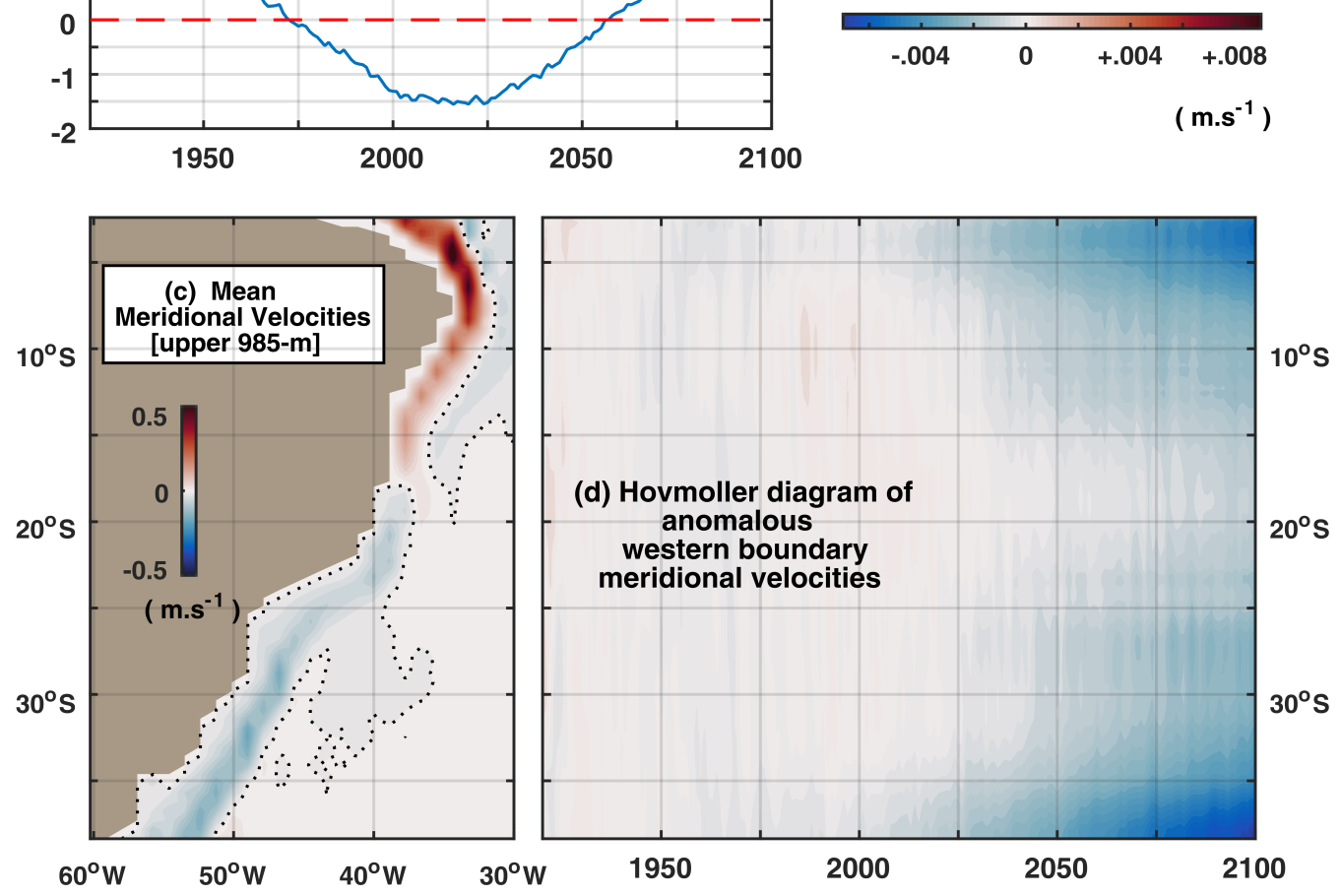

Figure 31 | Changes in the distribution of zonal and meridional SASG flows | (a) Spatial pattern of the leading EOF $(75.8 \%$ of total variance) of linearly detrended zonal velocity anomalies (1920-1970 base period) during 1920-2100 within the upper 985-m of the South Atlantic subtropical gyre (delimited by the 1920-2100 mean zero zonal velocity line - black filled contour). (b) Corresponding dimensionless amplitude, or Principal Component (PC), time series. c 1920-2100 mean, upper $985-\mathrm{m}$, meridional velocities along the South Atlantic western boundary, showing the north-south distribution of the western boundary currents, for reference. $\mathbf{d}$ Hovmoller diagram of anomalous meridional velocities averaged over the western boundary layer (within a $6^{\circ}$-longitude band off the South American coast), corresponding to the meridional extension in (c).

It might be possible that the detrended EOF actually points to a natural pattern of the SASG circulation variability, that reverses during 2000-2025 and acts to reinforce the externally forced pattern resulting from the non-detrended 
EOF. Or, it might be the case that externally forced signals potentially manifest even if the linear trend is previously removed from the data - either because internal variability itself might be influenced by external forcing (Maher et al., 2020), or even because the nonlinear detrending method used does not properly remove externally forced signals from the data (Vazquez, 2018).

Further investigation is needed to properly interpret these results, particularly with respect to the phasing reflected by detrended and non-detrended EOF amplitude time series.

However, considering that both EOF spatial patterns look virtually alike regardless of whether linear trends have been previously removed from the data or not -, from here on focus is given to the interpretation of this spatial pattern in terms of its dynamical implications.

The pattern clearly supports the previous results here presented: pointing to an intensification of the southern SASG circulation (featuring the increased westward AL flow upstream as well as the increased southern and central SSEC and $\mathrm{BC}$ flows downstream) during the RCP8.5 projected period, as opposed to a weakening of the northern SASG circulation, along its northern limb, and of the northward western boundary NBUC flow north of the SBL region.

Figure $31 \mathrm{~d}$ consists of the evolution in time of the meridional velocity anomalies with respect to the climatological north- and southward western boundary currents along South America (within a $6^{\circ}$-longitude band off the coast), which are shown in Figure 31c, for reference.

The developing of negative (i.e., southward) anomalous flows both to the north and south of the mean SBL region (see the black circle in Figure 21b for reference) further supports the projected subtropical BC strengthening and NBUC/AMOC upper limb weakening. It is shown that diffuse and weak anomalies emerge from the 2000s, starting at the northern and southernmost edges of the domain. After the year of 2050, the NBUC and BC anomalies propagate meridionally, spreading towards each other - which means that initially low/highlatitude ending portions of the NBUC/BC first start to decrease/increase; then gradually the NBUC/BC is weakened/strengthened towards its site of origin, at initial portions, albeit in a smaller magnitude when compared to ending portions. 
It is also interesting to note that the onset of anomalies in Figure $\mathbf{3 1} \mathbf{d}$ also coincides with the period of phase shifting of the EOF in Figures $\mathbf{3 1} \mathbf{a}, \mathbf{b}-$ by entering the RCP8.5 period. Again, it is uncertain whether the external forcing influences the internal climate variability somehow, or if the externally-forced signal is still present in the EOF pattern, even if the linear detrending technique has been previously performed.

The anomalous pattern of the sea surface height (SSH) field is shown in Figure $\mathbf{3 2} \mathbf{b}$, aside the climatological SSH field, for reference (Figure $\mathbf{3 2} \mathbf{a}$ ).
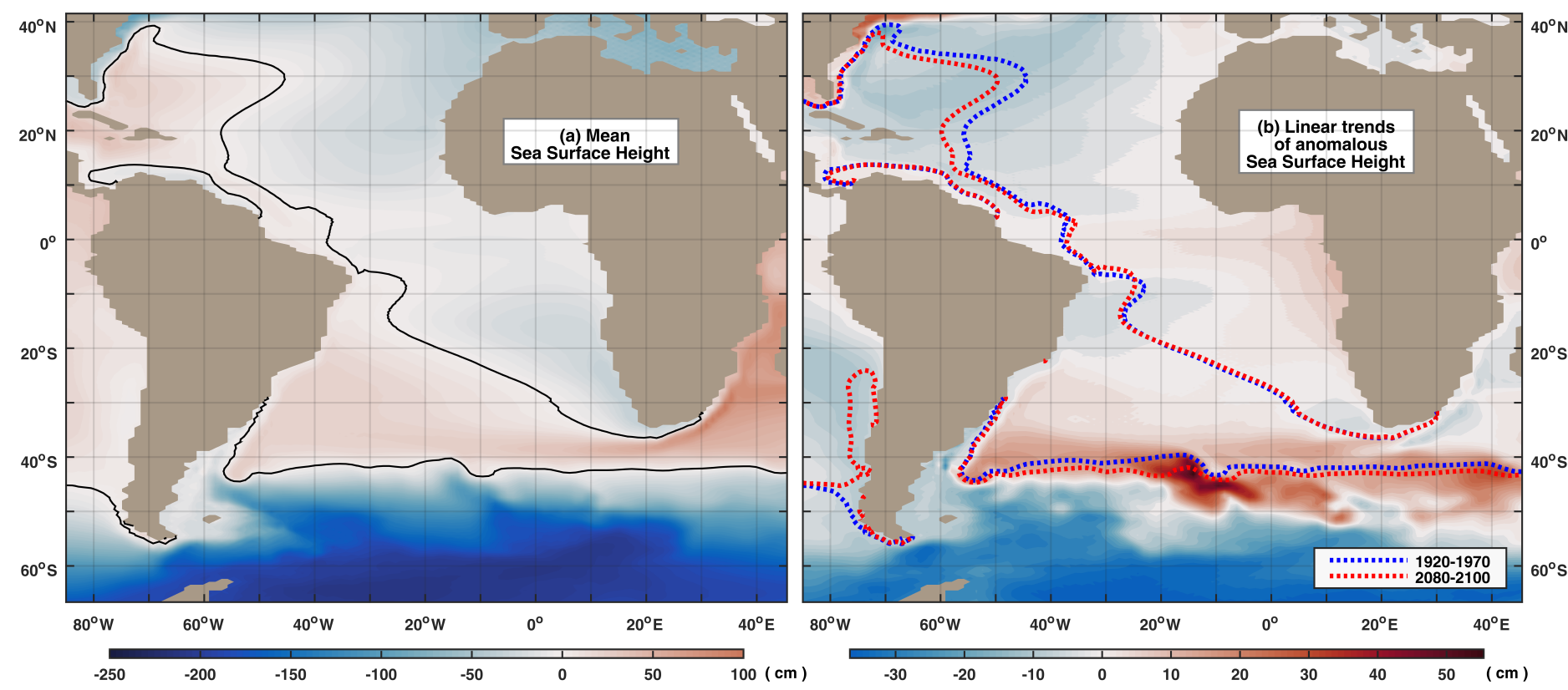

Figure 32 | SSH mean and anomalous 1920-2100 total trends | (a) 1920-2100 mean sea surface height (SSH). Red (blue) represents positive (negative) SSH values and the black filled contour marks the zero SSH line. (b) 1920-2100 total trends of SSH anomalies (1920-1970 base period). Red (blue) background colors represents positive/increasing (negative/decreasing) trends, while the red (blue) dotted contour represents the mean 1920-1970 (2080-2100) zero SSH line.

The SSH anomalies somehow also corroborate the observed changes inferred from the horizontal velocity fields. A spinned-up subtropical gyre circulation is associated with increased SSH values (e.g., Marcello et al., 2018) - which are observed right at the region where the AL-BeC-central/southern SSEC-BC system strengthens. North of that, negative SSH anomalies mark the region at the northern boundary of the SASG and at the NBUC region - where the horizontal flows are weakened. A southward shift of the SASG southern boundary is also 
demonstrated by the red/blue dotted contours - in this case, the shift extends across the basin, up to the Indian sector.

\subsection{Conclusions and Discussion}

This study focused on describing a projected scenario of circulation changes for the upper-ocean pathways of the South Atlantic overturning circulation, as according to the simulation results of the CESM1-Large Ensemble under the high-end RCP8.5 scenario.

The 40-member CESM-LE ensemble mean averages out natural, internal climate variability and thus represents the forced response - which is dominated by anthropogenically forced climate change. This is particularly important, given that any single model contains both intrinsic and externally forced contributions and isolating their relative effects requires an ensemble of simulations with a single climate model in which each ensemble member is subject to the same external forcing (historical from 1920 to 2005 and RCP8.5 thereafter, until 2100).

The results here presented support the recently debated weakening of the AMOC (according to the literature cited on Section 5.1), further providing modeling evidence for a meridionally coherent AMOC weakening along the Atlantic basin. But, most importantly, these results illustrate a projected scenario for changes in circulation pathways upstream of the North Atlantic - where the vast majority of research efforts are focused -, showing that in the South Atlantic basin the weakened transport along the AMOC upper limb flow happens in favor of a strengthened SASG circulation - instead of as a consequence of reduced cold and/or warm water route transports, for instance, which could also be the case.

Under a high emission scenario, we find a projected end of 21st century ensemble mean South Atlantic circulation change pattern, featuring a stronger and southward-shifted anticyclonic SASG circulation, at the expense of a weakened northward advection downstream of the SASG, along the western boundary NBUCNBC system - which is the main cross-equatorial conduit for the AMOC upper limb.

More specifically, by following up- to downstream overturning circulation pathways, it is found an increased transport through the DP, extending northward 
into the South Atlantic basin through the MC. By meeting a likewise increased $\mathrm{BC}$, at its southern termination, the Brazil-Malvinas Confluence gives rise to a conspicuously strengthened SAC at the southern limb of the SASG (Figure 25 g). While the eastward SAC transport along $\sim 45^{\circ}$ S tends to increase up to $20^{\circ} \mathrm{W}$, circulation changes east of that are marked by strong interplay between the SAC and the Southern Ocean ACC (Figure 21d).

Although the southward AC off the African east coast weakens, the $A L$ extension into the SASG strengthens, translating into an intensified westward corridor along $\sim 38^{\circ} S$ crossing the South Atlantic towards the western boundary and feeding directly the southern termination of the BC (Figures 26h, 21 d). Despite being out of the range typically defined for the wide SSEC flow, we attribute the westward transport around this latitudinal band to a southern portion of the SSEC.

North of that, the westward transport crossing the basin along the central SSEC shows no long-term trend, and the northern sSEC (delimiting the SASG northernmost boundary) displays a subtle long-term decreasing trend (Figures 26h and 28d).

Meridionally, off the South American coast, the northward NBUC undergoes a pronounced decrease in transport (Figure 26h) - which extends all the way into the northern North Atlantic along the AMOC upper limb path (Figure 21d). The MOC streamfunction at consecutive sections from the southern South

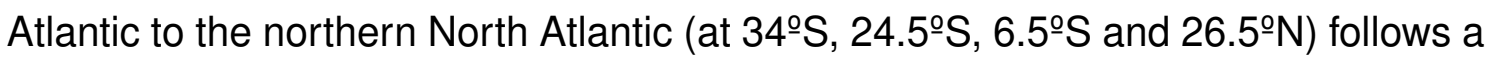
weakening trend, virtually at the same rate of that of all three NBUC transports, displaying a meridionally coherent AMOC slowdown along the Atlantic basin (Figure 26h).

However, when accounting only for northward transbasin transports (not discounting southward return flows) it is shown that across $34^{\circ} S$ the northward transports do not decrease during the whole period - differently than across the $24.5^{\circ} \mathrm{S}, 6.5^{\circ} \mathrm{S}$ and $26.5^{\circ} \mathrm{N}$ sections. This means that the MOC streamfunction at this section (34으) decreases owning to the strengthened southward $B C$ flow; while the northward component of the BeC-sSEC system does not decrease across this latitude (Figure 26h).

A northward displacement of the SBL (Figure 30 ) supports the fact that 
even though the northern SSEC does not decrease at a rate comparable to that of the NBUC, the westward SSEC flow is being unfairly distributed, favoring the southward $B C$ and recirculating in the SASG - meaning that even central and northern portions of the SSEC which are generally prone to feed the northward NBUC are actually being relocated to turn southward in their majority.

Besides the AMOC weakening, a shallowing trend of the AMOC upper limb is depicted both by Figures 27e-h and 30. Through Figures 27e-h it is shown that this shallowing occurs from the 2000s onward, with an accelerated rate after 2050.

With respect to structural changes in SASG spatio-temporal characteristics, while its northwestern boundary (off the South American coast) is projected to shift northward (mainly below $150 \mathrm{~m}$ - Figure 30) according to the zero meridional velocity line which defines the SBL, near the southwestern boundary the westward southern sSEC flow spreads southward (Figure 29a), featuring a southward shift of the zero zonal velocity line at this region.

At the SASG center (between $30^{\circ}-10^{\circ} \mathrm{W}$ - Figure 28a, d), the whole westward SSEC migrates to the south, according to the zero zonal velocity line implying a systematic southward shift of the westward northern limb of the SASG at the middle of the SAO basin — far from the western boundary.

In sum, considering the northern, westward counterpart of the anticyclonic SASG circulation (i.e., the whole SSEC), the results point to a equatorward-topoleward expansion of its $\sim$ deeper-northern (Figure 30) and $\sim$ surface-southern (Figure 29) limits, respectively, at the western SASG; along with a systematic poleward migration of both its northern and southern limits at the SASG center, to the east (Figure 28a). In other words, the SASG northern limb is projected to change its structure non-homogeneously across the zonal-depth plane - characterizing a vertically tilted latitudinal expansion of its western portion and a systematic southward shift of its central portion.

The EOF analysis of both detrended and non-detrended zonal velocity anomalies for the SASG domain (within the mean zero zonal velocty line) display nearly identical spatial patterns (Figures 31 and 38) - reflecting increased westward $<$ AL-southern/central SSEC $>$ and southward BC flows, contrasted with a 
decreased northern SSEC flow within the SASG for the RCP8.5 period. The difference between the detrended and non-detrended EOFs lies on the PC/amplitude time series: the non-detrended EOF points to a linear reinforcement of this pattern from 1920 to 2100 whereas the detrended EOF points to a phase shift straddling 2000-2005 (i.e., the transition from the historical 20TR to the RCP8.5 period). The EOF spatial pattern also reveals a weakening of the outer $A L$ flow, while its inner portion increases.

Tied to the EOF analysis, a Hovmoller diagram of north-to-south western boundary meridional velocity anomalies shows the onset of negative anomalies both with respect to the north- and southward WBCs (i.e., implying a northward NBUC decrease and southward BC increase) from the 2000 onward - matching the timing of the detrended EOF phase shift. This suggests that either external forcings might be influencing internal climate variability (Maher et al., 2020) or that the linear-detrending technique does not efficiently remove the externally-forced signal (Vazquez, 2018), or even that natural patterns of internal climate variability act to reinforce the climate change pattern by entering the RCP8.5 period.

Analysis of the eastward transports along the Southern Ocean, from the Pacific sector (prior to the DP) to the initial Indian sector (leaving the South Atlantic basin sector) show that northern (southern) eastward flows tend to weaken (strengthen). This suggests a strengthening of the ACC, leaving weak eastward flows north of it, along the South Pacific, South Atlantic and Indian oceans bordering the SHSG.

Finally, 1920-2100 total trends of the SSH field support a strengthening (weakening) of the southern (northern) SASG circulation. It is also found a southward expansion of the zero SSH line along the southern SASG, extending to the Indian basin (as an expression of the SHSG connectivity).

The results here presented are in fair agreement with other simulation and observational results recently reported in the literature. For example, the CESM1LE projected change pattern in the Agulhas system (Figure 21d) resembles the one recently found by Gupta et al. (2021) with CMIP5/CMIP6 simulations - where a projected decreased in the $A C$ is shown, whereas the $A C$ extension intensifies. Here, the $A C$ extension region also weakens, but the westward $A L$ component 
strengthens. The authors also support a projected strengthening of the poleward $B C$ and weakening of the cross-equatorial NBC transport (see their Figure 1 for a visual representation of their results). A projected weakening of the $A C$ transport in a warming climate is also reported by Ma et al. (2020).

Durgadoo et al. (2013) showed that the simulated wind-driven increase in the AL happens along with a strengthening of the SASG, arguing that this strengthening leads to a favored recirculatory route at the $S B L$, resulting in less $A L$ waters feeding into the NBC as part of the AMOC upper limb. The results of Rühs et al. (2019) are also in agreement with this theory. Beal and Elipot (2016), in turn, argue that the intensification in the global wind systems has acted to increase the eddy activity which in turn translates into a broadening of the $A C$ since the early 1990s, instead of an increase in its transport. Using observational datasets, the recent study of Martínez-Moreno et al. (2021) also supports a global-scale enhancement of the oceanic mesoscale eddy kinetic energy over the past three decades, as a readjustment of the surface ocean circulation to a changing climate.

Drouin et al. (2021) also finds a significant southward migration of the SASG southern boundary along 1993-2018, based on observational data - although the authors do not find trends in gyre size or strength.

A strengthening of the SPSG is supported by various studies (Cai, 2006; Roemmich et al., 2007; Schneider et al., 2007; Zhang and Qu, 2015), with an increase of the equatorward transport in its eastern portion reported by Roemmich et al. (2016), using Argo and hydrographic data. This is in line with our results, in that less waters are escaping to the east at the southeastern base of the gyre. Oliver and Holbrook (2014), however, suggest through 1990-2060 downscaled projections that it is not the entire SPSG that spins-up, but only the southern western boundary East Australian Current extension to the south, whereas the outflow along the eastward Tasman Front decreases, although only by $2.7 \mathrm{~Sv}$.

A projected intensification of the $\mathrm{AL}$, linked via interior pathways to an even greater increase in the BC strength (40\%) is found by Pontes et al. (2016), using CMIP5 model simulations. They also report a decrease in the northern South Atlantic basin interior and in the northward NBC transport along the AMOC upper limb; besides a southward shift of the BMC at the southwestern SASG boundary. 
Qu et al. (2019) report a generalized strengthening of subtropical gyres in all three SH oceans (i.e. the SHSG), since 1993, using observational data and results from an ocean state estimate.

Using reanalysis products, satellite observations and CMIP5 model simulations, Yang et al. (2016) found a strengthening and poleward shift of global WBCs - also attributed to wind-driven changes in response to positive annular mode-like trends. The GS is the exception, which is expected to weaken along with the AMOC upper limb.

Considering that basin-scale AMOC variability is superimposed by winddriven gyre variability, and that gyre dynamics have the potential to impact the properties shaping AMOC upper limb waters, this study provides important insights into the up- to downstream transmission of low-frequency circulation signals that in turn might translate to changes in fundamental thermohaline properties involved in the global overturning circulation. Up to now, no study has approached long-term projected changes in South Atlantic upper-ocean circulations, let alone from the perspective of single-model large ensemble simulations.

The manuscript related to this Chapter (5) is still being prepared for submission. Next steps consist in addressing model validation, investigating associated changes in the wind stress curl field and performing 20TR/RCP8.5 comparisons with the CESM1-LE pre-industrial control run. 


\section{Concluding Remarks}

The South Atlantic large-scale circulation and dynamics have been addressed throughout this thesis from different view points in space and time.

The investigations presented in Chapters 3, 4 and 5, despite having particular outlooks and approaches, are all based on and motivated by the role of the South Atlantic in the context of the Global Overturning Circulation, involving the disruption of commonly overvalued meridional pathways by frequently overlooked horizontal gyre circulations. Essentially, basin-scale dynamics associated with the SASG and the AMOC upper limb are explored in the context of past low-frequency climate variability and future climate change.

Chapter 3 builds upon existing research suggesting recent changes in the circulation of global subtropical gyres with respect to the SASG using simulation results from the CESM1-POP2 from the late 20th into the early 21st century. Systematic changes were found, that potentially reflect the superposed influence of changing atmospheric dynamics and buoyancy-driven AMOC variations.

More specifically, the whole SASG is shown to be concurrently spinning-up and drifting poleward - two outcomes which are generally attributed to recently reported Southern Hemisphere shifted wind patterns. However, on the interbasin scale, a southward extension of the joint subtropical SHSG is usually described for its southern boundary - associated with the migration of the zero wind stress curl line extending across all three ocean sectors (e.g., Cail, 2006).

Theoretically, a strengthening of the SASG circulation and consequently of the western boundary BC transport imply a northward SBL (e.g., Rodrigues et al., 2007), which disagrees with a southward displacement of the SASG northern boundary in conjunction with the whole gyre. Yet, in this study, despite of an increased anticyclonic SASG circulation (tied to an enlargement of its core), an intensification of the flow along the main conduits of the AMOC upper limb is also found (the sSEC-NBUC system) - in association with an increasing trend of the AMOC streamfunction at $26.5^{\circ} \mathrm{N}$.

Thereby, the northward NBUC transport increase is of greater magnitude than the modest increase in the southward BC transport - indeed resulting in a 
southward shifted SBL at the northwestern boundary of the SASG.

Even though the CESM1-POP2 simulates a linearly increasing AMOC strength, this trend is embedded in substantial multidecadal variability, which is generally in agreement with directly observed $26.5^{\circ} \mathrm{N}-\mathrm{AMOC}$ oscillations, as discussed in Section 3.3. Moreover, it has been demonstrated that on interannual and decadal timescales, the AMOC variability in CORE-II simulations with prescribed atmospheric state (as is the case of this study, in which the POP2 ocean model is forced with the CORE-II interannual atmospheric data set) is in reasonable agreement with the atmospheric variability represented in coupled CMIP5 simulations (Xu et al., 2019).

It should be noted, however, that the period covered by this study is too short to permit conclusions about long-term AMOC changes to be drawn, especially considering the potentially significant role played by natural multidecadal variability (Roberts et al., 2014; Worthington et al., 2021; Srokosz and Bryden, 2015; Jackson et al., 2016; Collins et al., 2013).

Chapter 4 provides a longer-term context for modern changes, showing that in warm and cold climate extremes of the pre-industrial past millennium the South Atlantic western boundary current system responded by redirecting the westward SSEC flow arriving at the South American coast preferably to the north and south, respectively, favoring either the AMOC upper limb or the SASG circulation.

While a consistent picture of AMOC variability over the last millennium is still lacking (Kilbourne et al., 2016; Moffa-Sánchez et al., 2019), some studies provide evidence for a relatively strong AMOC around the warm MCA and a weaker AMOC during the cold LIA (Wanamaker et al., 2012; Trouet et al., 2009, 2012; Lund et al., 2006).

The climate oscillations of the last millennium and therefore the contrasting MCA and LIA climatic episodes were fully established by natural forcings and internal variability. The scenario illustrated by the 10-member CESM-Last Millennium Ensemble mean represents the climate system forced response - in this case to the natural external forcings that operated over the $\sim 850-1850$ millennial period.

The MCA is considered the most recent counterpart to modern warmth, 
yet, a strong AMOC during the warm MCA period is inconsistent with the (even warmer - Neukom et al., 2019) human era AMOC decline suggested by the literature. Nevertheless, as previously emphasized in this thesis, the AMOC is not a linear system, which makes it so difficult to reliably simulate and predict its response. The possibility of other driving mechanisms for millennial-scale climate variability also can not be excluded - such as the influence of solar irradiance and volcanic forcing (Moffa-Sánchez et al., 2019); furthermore, factors such as timing, climate feedbacks and spatial coherence further complicate this matter.

Finally, Chapter 5 illustrates a possible future scenario for ocean circulation changes that concern upper-ocean pathways of the South Atlantic overturning circulation, comprehensively involving the cold and warm water routes entering both opposing corners of the South Atlantic basin, the SASG circulation, and the northward passageway of the AMOC upper limb through the NBUC-NBC.

The forced response of the climate system (i.e., the response to humaninduced climate change) is represented by the CESM1-LE ensemble mean revealing striking projected long-term changes in up- to downstream volume transports. The simulated and projected AMOC weakening (supported by the literature referenced in Section 5.1) is corroborated by the CESM1-LE results, and projected changes occurring upstream of the North Atlantic MOC are presented. It is shown that while the circulation at the northern SASG and through the NBUC-NBC are weakened, the SASG recirculation through the poleward BC is strengthened - actually featuring a core intensification similar as that reported in the results from Chapter $3 /$ Marcello et al. (2018).

Figure 33 together with Figure 34 provide an inclusive perspective of the three model simulations used in this thesis. The surface SBL time series is used to compare its low-frequency variability along the periods covered by each simulation: the one with the ocean component CESM1-POP2 (upper panel - associated with Chapter 3), with the CESM1-Last Millennium Ensemble (middle panel - associated with Chapter 4) and with the CESM1-Large Ensemble (lower panel - associated with Chapter 5). The full CESM1-LME period is shown (850-2005) to permit a comparison with the other model simulations, instead of only the period used in the investigation from Chapter 4 (850-1850). 
Interestingly, all CESM1 simulations are somehow in fair agreement and match each other in terms of the timing of the SBL low-frequency variability. The southward shifting period depicted by the CESM1-POP2 fits well within overlapping periods of southern SBL trends of the CESM1-LME and CESM1-LE.

A direct comparison between the CESM1-LME and the CESM1-Large Ensemble (CESM-LEns / CESM1-LE) is not shown, however, it is reasonable to conclude they are also in good agreement - with the 1920-1970 "CESM-LEns" period (lower panel - the blue portion of the time series prior to the orange portion which represents the CESM1-POP2 period) - that is common to the CESM1-LME undergoing an abrupt southward shift and rapidly recovering thereafter, from where it follows a long-term northward migration up to the 1970s. A similar long-term oscillation can be found for the CESM1-LME in the middle panel - right before the orange portion of the time series which represents the CESM1-POP2 period.
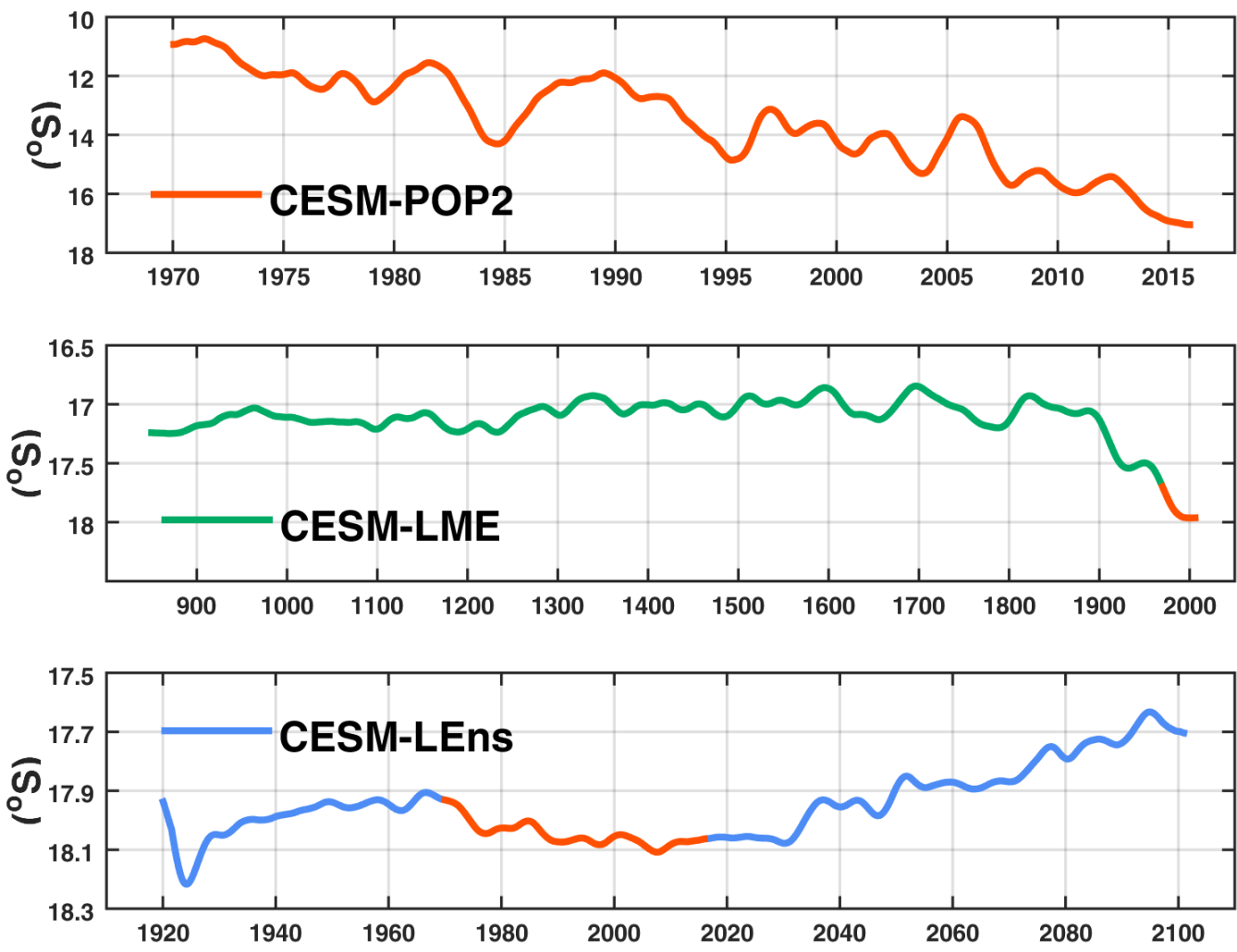

Figure 33 | Surface SBL time series from CESM1 simulations - the ocean-only POP2, the Last Millennium Ensemble and the Large Ensemble | The period corresponding to the CESM1POP2 SBL time series (orange, upper panel) is highlighted in orange in the CESM1-LME (green, middle panel) and in the CESM1-LEns (or CESM1-LE as previously referred to) (blue, lower panel) time series, for a temporal perspective. The $y$-axis indicates the range for the SSEC bifurcation latitude, according to each simulation. 


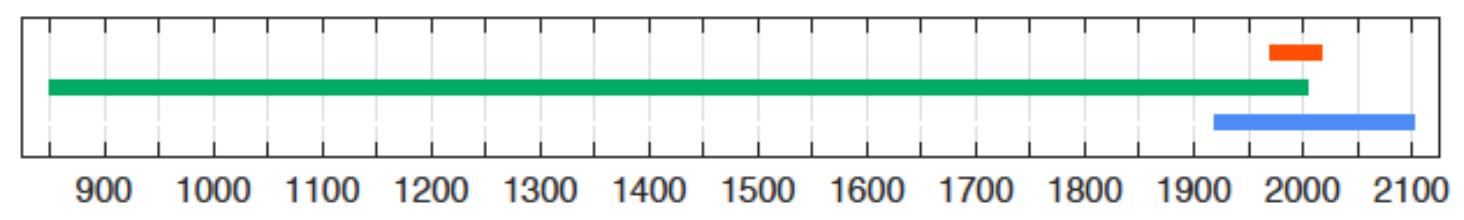

Figure 34 | Comparative time range of each model simulation shown in Figure 33 | Orange indicates the CESM1-POP2 1970-2015 period, green indicates the CESM1-LME 850-2005 period and blue indicates the CESM1-LE 1920-2100 period.

All results presented in this thesis should be interpreted with due caution, as they are obviously limited by the fidelity of the models employed. Dynamical processes and circulation changes here described are specific to the respective models and simulations associated with the chapters, and are therefore influenced by their particular biases. Primary caveats concern coarse resolutions (for instance, relevant $A M O C$ processes are sensitive to resolution and thus imperfectly captured by many current climate models) and the inability to resolve oceanic mesoscale processes and eddy features - which rely on parameterizations. Studies using independent models as well as observation-based and proxy data are highly desirable for confirming our findings. 


\section{Scientific Progress}

There is now unequivocal evidence for human-induced global warming and consequent climate change, which are modulated by natural drivers and internal climate variability - especially at regional scales and in the near term, with little effect on centennial global warming. Global temperatures will continue to rise until at least the mid-21st century under all emissions scenarios considered, and global warming of $1.5^{\circ} \mathrm{C}$ and $2^{\circ} \mathrm{C}$ will be exceeded during the 21 st century unless deep reductions in $\mathrm{CO}_{2}$ and other GHGs emissions occur in the coming decades (The IPCC Sixth Assessment Report - MassonDelmotte et al., 2021).

In view of the current and projected climate scenario facing the Earth system and humanity, and in view of the large uncertainties in our current understanding of the future unfolding of the general atmospheric and oceanic circulations - including AMOC variability and predictability -, it is crucial to seek for "accumulation of scientific knowledge" in order to reach knowledge-based consensus on important climate science topics which are naturally susceptible to the problem of subjectivity.

For example, regarding AMOC changes, it is still unclear whether available time series are simply too short to confirm mechanisms acting on longer timescales or whether existing assumptions on the AMOC need to be revised. Therefore, it is still unconsolidated whether the recent observed AMOC decline is due to natural decadal oscillations or if it is indeed a climate change forced response. Just as it is even more uncertain how will AMOC dynamics evolve over the coming century and beyond.

Science depends on evidence coming together and telling the same story. While there are several different lines of evidence pointing to the rise of globally averaged temperatures, there is substantial lack of knowledge concerning how the climate system components are to interact causing heat to move within the climate system itself, assuming other forms of energy that flow altering weather and climate patterns - shaping the state, variability and change of Earth's climate. 
Considering the need to understand the role of the ocean in climate change evolution and projections, and considering that the South Atlantic is a critical region for processes that maintain the global overturning circulation, the scientific progress made by the investigations composing this thesis are punctuated:

\section{Chapter 3:}

Based on the CESM1-POP2 simulation results for the period of 1970-2015:

- The SASG has strengthened and shifted southward - following a global expression of midlatitude (especially Southern Hemisphere) subtropical gyres in response to anthropogenic climate change;

- The SASG basin-scale wind stress curl, sea surface height and barotropic stream function have intensified;

- The northwestern boundary of the SASG - represented by the SBL - has shifted poleward in association with an increased equatorward transport along the NBUC (i.e., along the AMOC upper limb);

- The AMOC streamfunction at 26.5ㅇN has undergone a linear increasing trend, which is mediated by multidecadal variability;

- The overall SASG circulation changes found reflect an interplay of winddriven as well as buoyancy-driven forcings under global warming - making the SASG unique among global subtropical gyres.

\section{Chapter 4 :}

Based on the CESM1-Last Millennium Ensemble simulation results for the period of 850-1850:

- The subtropical western South Atlantic surface circulation pattern displays contrasting responses to warm and cold climate extremes of the last millennium, which are associated with periods of enhanced and weakened AMOC, respectively; 
- During the warm MCA (cold LIA), a weaker (stronger) anticyclonic subtropical gyre circulation and stronger (weaker) equatorward AMOC upper limb transport is inferred;

- The results inferred from the horizontal velocity fields are further supported by changes in the sea surface temperature and wind stress curl fields;

- The MCA/LIA differences described are in agreement with other large-scale changes reported in the literature for these anomalous periods.

\section{Chapter 5 :}

Based on the CESM1-Large Ensemble simulation results for the period of 19202100:

- A projected end of 21 st century forced response of South Atlantic overturning circulation upper-ocean pathways is expressed by the 40 -member mean of a single-model large ensemble under a high emission scenario;

- Results support the recently observed/simulated and future projected AMOC decline under externally forced global warming - featuring a shallowing AMOC upper limb and meridionally coherent decreasing AMOC streamfunctions from the southern South Atlantic to the northern North Atlantic;

- Upstream of the northern North Atlantic, it is shown that the weakened transport along the AMOC upper limb in the South Atlantic sector gives place to a strengthened SASG circulation;

- Transport contributions from the Pacific cold water and Indian warm water routes to the South Atlantic circulation do not decrease, however, these are preferably redirected to recirculate in the subtropical gyre instead of following the northward AMOC upper limb;

- The SASG spatial structure is projected to change non-homogeneously across the zonal-depth plane - with a tilted expansion of its western portion and a systematic southward drift of its central portion; 
- The southern eastward ACC flow is projected to increase from the Pacific to the Indian sector of the Southern Ocean, across the Atlantic sector - leaving weak northern eastward flows at the base of the joint Southern Hemisphere supergyre.

- The presented simulated and projected changes provide further modeling evidence and complement important pieces of information with respect to the recent literature reporting current and future ocean circulation changes in response to anthropogenic climate change. 


\section{Other publication results}

\subsection{Warm Deep Water variability during the Last Millennium in the CESM-LME: pre-Industrial scenario vs. late 20th cen- tury changes}

Water transformation around Antarctica is recognized to significantly impact the climate. It is where the linkage between the upper and lower limbs of the MOC takes place by means of dense water formation, which may be affected by rapid climate change. Simulation results from the CESM-LME are used to investigate the Weddell Sea Warm Deep Water (WDW) evolution during the LM. The WDW is the primary heat source for the Weddell Sea (WS) and accounts for $71 \%$ of the Weddell Sea Bottom Water (WSBW), which is the regional variety of the AABW - one of the densest water masses in the ocean bearing directly on the cold deep limb of the MOC. Earth System Models (ESMs) are known to misrepresent the deep layers of the ocean (below $2000 \mathrm{~m}$ ), hence we aim at the upper component of the deep meridional overturning cell, i.e., the WDW. Salinity and temperature results from the CESM-LME from a transect crossing the WS are evaluated with the Optimum Multiparameter Analysis (OMP) water masses decomposition scheme. It is shown that, after a long-term cooling over the LM, a warming trend takes place at the surface waters in the WS during the 20th century, which is coherent with a global expression. The subsurface layers and. mainly. the WDW domain are subject to the same long-term cooling trend, which is decelerated after 1850 (instead of becoming warmer like the surface waters), probably due interactions with sea ice-insulated ambient waters. The evolution of this anomalous temperature pattern for the WS is clear throughout the three major LM climatic episodes: the MCA, LIA and late 20th century warming. Along with the continuous decline of WDW core temperatures, heat content in the water mass also decreases by $18.86 \%$. OMP results indicate shoaling and shrinking of the WDW during the LM, with a $\sim 6 \%$ decrease in its cross-sectional area. Although the AABW cannot be directly assessed from CESM-LME results, changes in the WDW structure and WS dynamics have the potential to influence the deep/bottom 
water formation processes and the global MOC.

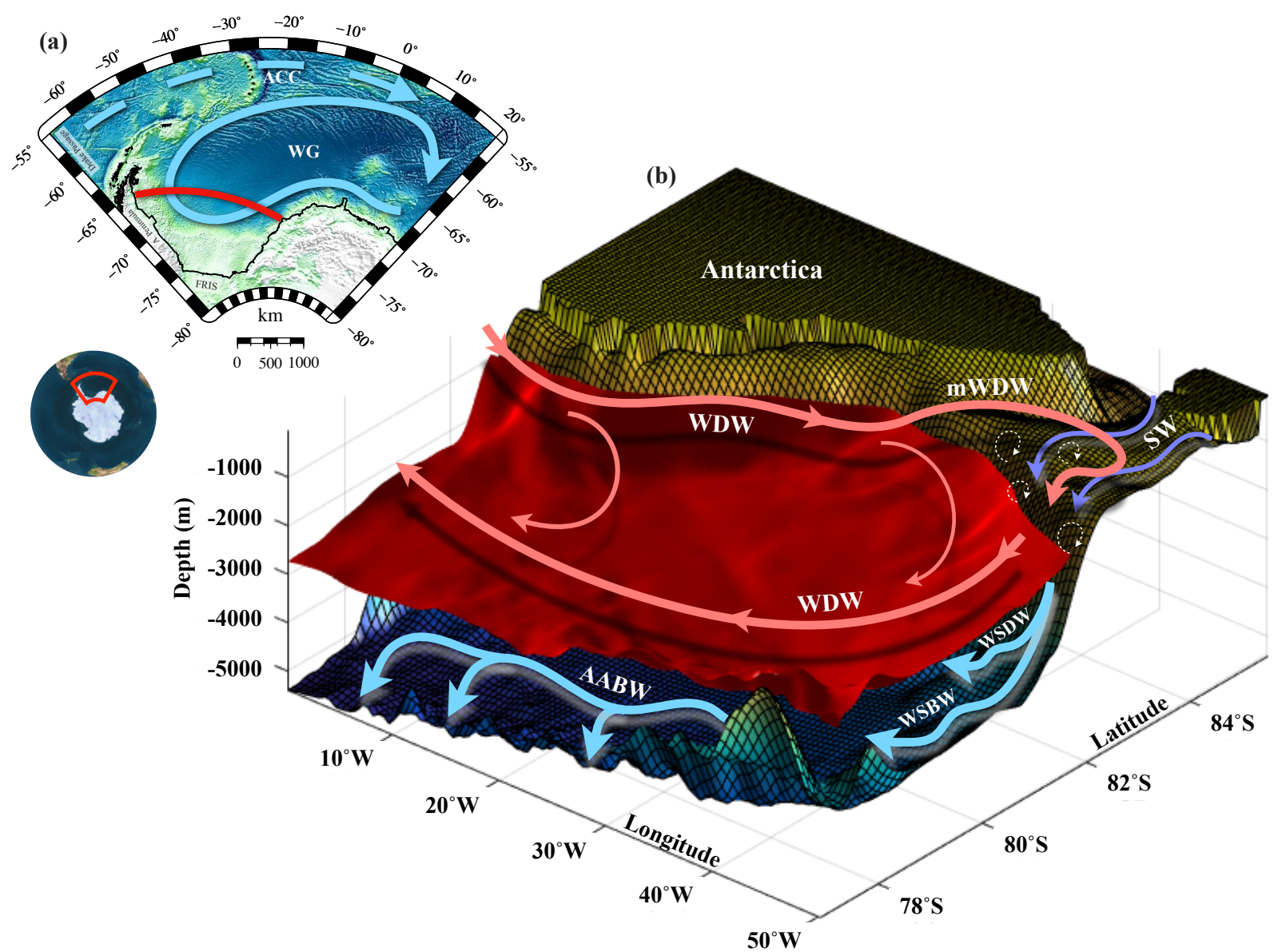

Figure 35 (a) Atlantic sector of the Southern Ocean topography centered at the Weddell Sea. The full blue arrow represents the Weddell Gyre (WG) circulation and the dashed blue arrow represents the Antarctic Circumpolar Current (ACC) flow. The red line marks the transect assessed with the OMP for WDW investigation throughout the LM, encompassing the inflow and outflow regions of the WG. (b) Perspective of the Weddell Sea multi-layer circulation, depicting the nearshelf break interactions between the WDW (coral arrow) and the super cooled Shelf Waters (SW-purple arrows) to form the WSDW and WSBW, ultimately exported as AABW (light blue arrows). 


\subsection{Long-term regional sea level changes from CMIP6 projec- tions}

Anthropogenic climate forcing will cause the global mean sea level to rise over the 21 st century. However, regional sea level is expected to vary across ocean basins, superimposed by the influence of natural internal climate variability. Here, we address the detection of dynamic sea level (DSL) changes by combining the perspectives of a single and a multi-model ensemble approach (the 50-member CanESM5 and a 27-model ensemble, respectively, all retrieved from the CMIP6 archive), under three CMIP6 projected scenarios: SSP1-2.6, SSP3-7.0 and SSP58.5. The ensemble analysis takes into account four key metrics: signal (S), noise $(\mathrm{N}), \mathrm{S} / \mathrm{N}$ ratio, and time of emergence (ToE). The results from both sets of ensembles agree in the fact that regions with higher $\mathrm{S} / \mathrm{N}$ (associated with smaller uncertainties) also reflect earlier ToEs. The DSL signal is projected to emerge in the Southern Ocean, Southeast Pacific, Northwest Atlantic, and the Arctic. Results common for both sets of ensemble simulations show that while $\mathrm{S}$ progressively increases with increased projected emissions, $\mathrm{N}$, in turn, does not vary substantially among the SSPs, suggesting that uncertainty arising from internal climate variability has little dependence on changes in the magnitude of external forcing. Projected changes are greater and quite similar for the scenarios SSP3-7.0 and SSP5-8.5 and considerably smaller for the SSP1-2.6, highlighting the importance of public policies towards lower emission scenarios and of keeping emissions below a certain threshold. 

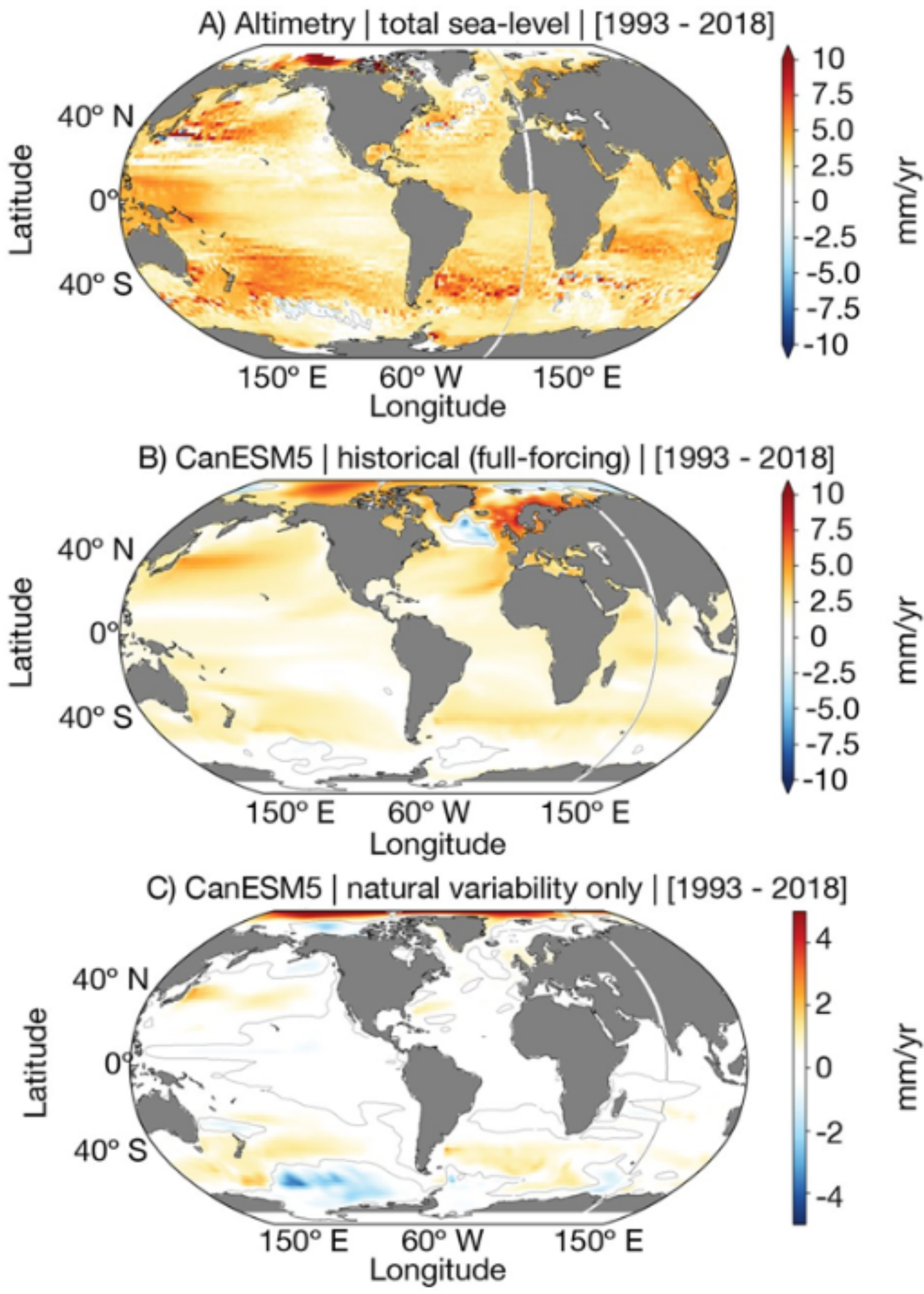

Figure 36 Annual mean linear trend (mm.yr ${ }^{1}$ ) for the years 1993-2018 of (a) total sea level from satellite observations (AVISO+), (b) sterodynamic sea-level (DSL plus global mean thermosteric sea level) from CanESM5 historical plus SSP585 (50 members average) and (c) from CanESM5 historical- natural (10 members average). The altimeter-derived sea levels refer to the ocean topography with respect to the geoid. 


\subsection{1st century projected changes in the Weddell Sea Antarc- tic Slope Current in a Downscaling Experiment}

The Antarctic Slope Current (ASC) and its associated frontal structure regulate the exchanges between shelf and ocean waters. Changes in their structure and variability can alter the heat budget over the Antarctic margins and impact processes globally. In this study we investigate the ASC frontal structure response to climate change in the Weddell Sea region from a high-resolution experiment with the Regional Ocean Model System (ROMS) forced by MPI- ESM-MR simulation results for the CMIP5 RCP8.5 scenario (business as usual). Analysis were performed in two regions with distinct dynamics located in the inflow and outflow of the Weddell Sea. Results show regional variation in the external forcings (winds and sea ice) and, therefore, different responses along the ASC system to climate change. Weakening in the ASC volume transport driven mainly by the decrease in the easterlies magnitude is observed at the inflow region. The outflow shows strengthening trend due to enhanced lateral density gradient caused by freshening over the continental shelf. The outflow shifts from a Dense Shelf to a Fresh Shelf regime by the end of the 21 st century. Changes in the ASC structure assessed by this study could be linked to accelerated sea ice melting and the reduction of the Antarctic Bottom Water formation.

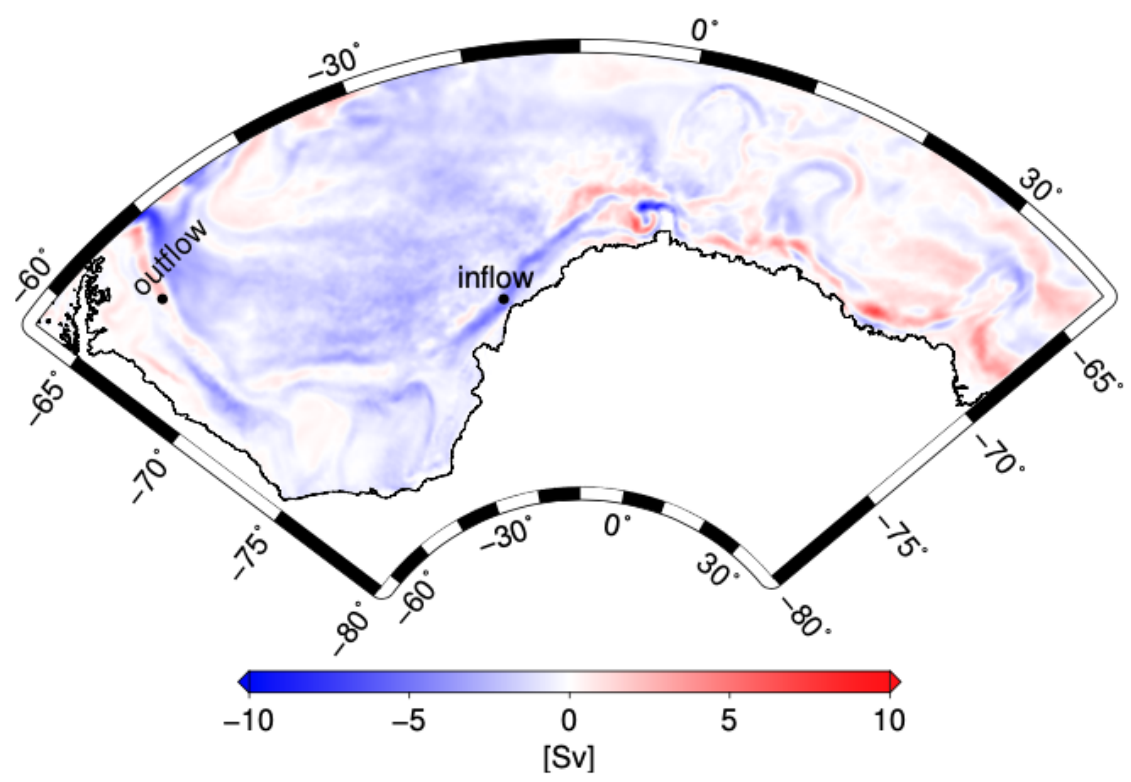

Figure 37 Climatological difference between the periods of 2091-2100 and 2020-2029 for the full-depth integrated volume transport. Difference values are significant at the $95 \%$ confidence level according to a t-test. Black dots illustrate the location of the inflow and outflow assessed regions. 


\section{List of Publications}

Publications associated with Chapters 3, 4, and 5, respectively:

- Marcello, F.; Wainer, I.; Rodrigues, R. R. South Atlantic subtropical gyre late twentieth century changes. Journal of Geophysical Research - Oceans (2018), 123. https://agupubs.onlinelibrary.wiley.com/doi/full/10.1029/2018JC013815

- Marcello, F.; Wainer, I.; Gent, P. R.; Otto-Bliesner B. L.; Brady, E. C. South Atlantic surface boundary current system during the Last Millennium in the CESM-LME: the Medieval Climate Anomaly and Little Ice Age. Climate Variability in Antarctica and the Southern Hemisphere over the Last Millennia - Geosciences (2019), 9, 299. https://doi.org/10.3390/geosciences9070299.

- Marcello, F.; Wainer, I.; Tonelli, M.; Ferrero, B. Upper-limb pathways of the South Atlantic Overturning Circulation in the CESM1-LE: low-frequency variability and projected changes. Manuscript in preparation.

Publications associated with Chapter 8.

- Tonelli, M.; Marcello, F.; Ferrero, B.; Wainer, I. Warm Deep Water variability during the Last Millennium in the CESM-LME: pre-industrial scenario versus late 20th-century changes. Climate Variability in Antarctica and the Southern Hemisphere over the Last Millennia - Geosciences (2019), 9, 346. https: //doi.org/10.3390/geosciences9080346.

- Ferrero, B.; Tonelli, M.; Marcello, F.; Wainer, I. Long-term Regional Dynamic Sea Level Changes from CMIP6 Projections. Adv. Atmos. Sci. 38, 157-167 (2021). https://doi.org/10.1007/s00376-020-0178-4.

- Noro, M.; Tonelli, T.; Marcello, F.; Ferrero, B.; Pereira, J. E.; Wainer, I. 21st Century Projected Changes in the Weddel Sea Antarctic Slope Current in a Downscalling Experiment. Manuscript in preparation. 


\section{References}

Abram, N. J., H. V. McGregor, J. E. Tierney, M. N. Evans, N. P. McKay, and D. S. Kaufman (2016). Early onset of industrial-era warming across the oceans and continents. Nature 536(7617), 411-418.

Alley, R. B. (2007). Wally was right: Predictive ability of the north atlantic "conveyor belt" hypothesis for abrupt climate change. Annu. Rev. Earth Planet. Sci. 35, 241-272.

Alory, G., S. Wijffels, and G. Meyers (2007, jan). Observed temperature trends in the Indian Ocean over 1960-1999 and associated mechanisms. Geophysical Research Letters 34(L02606), 1-6.

Arblaster, J. M., G. A. Meehl, and D. J. Karoly (2011). Future climate change in the southern hemisphere: Competing effects of ozone and greenhouse gases. Geophysical Research Letters 38(2).

Aricò, S., J. M. Arrieta, D. C. E. Bakker, P. W. Boyd, L. Cotrim da Cunha, F. Chai, M. Dai, N. Gruber, K. Isensee, M. Ishii, N. Jiao, S. K. Lauvset, G. A. McKinley, P. Monteiro, C. Robinson, C. Sabine, K. L. Sanders, R.; Schoo, U. Schuster, J. D. Shutler, H. Thomas, R. Wanninkhof, A. J. Watson, L. Bopp, E. Boss, A. Bracco, W. Cai, A. Fay, C. Heinze, S. Henson, J. Hwang, J. Post, P. Suntharalingam, M. Telszewski, B. Tilbrook, V. Valsala, and A. Rojas Aldana (2021). Integrated ocean carbon research: A summary of ocean carbon research, and vision of coordinated ocean carbon research and observations for the next decade. IOC Technical Series, 158, 46 pp.

Atwood, A., E. Wu, D. Frierson, D. Battisti, and J. Sachs (2016). Quantifying climate forcings and feedbacks over the last millennium in the cmip5-pmip3 models. Journal of Climate 29(3), 1161-1178.

Ault, T., C. Deser, M. Newman, and J. Emile-Geay (2013). Characterizing decadal to centennial variability in the equatorial pacific during the last millennium. Geophysical Research Letters 40(13), 3450-3456. 
Barker, S. and G. Knorr (2016). A paleo-perspective on the amoc as a tipping element. PAGES Magazine 24(1), 14-15.

Barnett, T., F. Zwiers, G. Hengerl, M. Allen, T. Crowly, N. Gillett, K. Hasselmann, P. Jones, B. Santer, R. Schnur, et al. (2005). Detecting and attributing external influences on the climate system: A review of recent advances. Journal of Climate 18(9), 1291-1314.

Beal, L. M., W. P. M. De Ruijter, A. Biastoch, and R. Zahn (2011, apr). On the role of the Agulhas system in ocean circulation and climate. Nature 472, 429-436.

Beal, L. M. and S. Elipot (2016). Broadening not strengthening of the agulhas current since the early 1990s. Nature 540(7634), 570-573.

Bellomo, K., M. Angeloni, S. Corti, and J. von Hardenberg (2021). Future climate change shaped by inter-model differences in atlantic meridional overturning circulation response. Nature Communications 12(1), 1-10.

Biastoch, A., C. W. Böning, J. Getzlaff, J.-M. Molines, and G. Madec (2008). Causes of interannual-decadal variability in the meridional overturning circulation of the midlatitude north atlantic ocean. Journal of climate 21(24), 65996615.

Biastoch, A., C. W. Böning, F. U. Schwarzkopf, and J. R. E. Lutjeharms (2009, nov). Increase in Agulhas leakage due to poleward shift of Southern Hemisphere westerlies. Nature 462, 495-498.

Blunden, J. and D. Arndt (2020). State of the climate in 2019. Bulletin of the American Meteorological Society 101(8), S1-S429.

Boebel, O., R. Davis, M. Ollitrault, R. Peterson, P. Richardson, C. Schmid, and W. Zenk (1999). The intermediate depth circulation of the western south atlantic. Geophysical Research Letters 26(21), 3329-3332.

Boers, N. (2021). Observation-based early-warning signals for a collapse of the atlantic meridional overturning circulation. Nature Climate Change 11(8), 680-688. 
Bower, A., S. Lozier, A. Biastoch, K. Drouin, N. Foukal, H. Furey, M. Lankhorst, S. Rühs, and S. Zou (2019). Lagrangian views of the pathways of the atlantic meridional overturning circulation. Journal of Geophysical Research: Oceans 124(8), 5313-5335.

Bower, A. S., M. S. Lozier, S. F. Gary, and C. W. Böning (2009). Interior pathways of the north atlantic meridional overturning circulation. Nature 459(7244), 243-247.

Bradley, R. S., K. R. Briffa, J. Cole, M. K. Hughes, and T. J. Osborn (2003). The climate of the last millennium. In Paleoclimate, global change and the future, pp. 105-141. Springer.

Broecker, W. S. (1987a). The biggest chill. Natural History Magazine, 74-82.

Broecker, W. S. (1987b). Unpleasant surprises in the greenhouse? Nature 328(6126), 123-126.

Broecker, W. S. (1991). The great ocean conveyor. Oceanography 4(2), 79-89.

Broecker, W. S. (1997). Thermohaline circulation, the achilles heel of our climate system: Will man-made co2 upset the current balance? Science 278(5343), 1582-1588.

Broecker, W. S. (1999). What if the conveyor were to shut down? reflections on a possible outcome of the great global experiment. GSA Today 9(1), 1-7.

Broecker, W. S. and G. H. Denton (1990). The role of ocean-atmosphere reorganizations in glacial cycles. Quaternary science reviews 9(4), 305-341.

Broecker, W. S., D. M. Peteet, and D. Rind (1985). Does the ocean-atmosphere system have more than one stable mode of operation? Nature 315(6014), 21-26.

Bryden, H., B. A. King, G. D. McCarthy, and E. McDonagh (2014). Impact of a 30\% reduction in atlantic meridional overturning during 2009-2010. Ocean Science 10(4), 683-691. 
Bryden, H. L., C. Robinson, and G. Griffiths (2012). Changing currents: a strategy for understanding and predicting the changing ocean circulation. Philosophical Transactions of The Royal Society A 370, 5461-5479.

Buckley, M. W. and J. Marshall (2016). Observations, inferences, and mechanisms of the atlantic meridional overturning circulation: A review. Reviews of Geophysics 54(1), 5-63.

Cabos, W., D. V. Sein, J. G. Pinto, A. H. Fink, N. V. Koldunov, F. Alvarez, A. Izquierdo, N. Keenlyside, and D. Jacob (2016, aug). The South Atlantic Anticyclone as a key player for the representation of the tropical Atlantic climate in coupled climate models. Climate Dynamics, 1-19.

Caesar, L., G. McCarthy, D. Thornalley, N. Cahill, and S. Rahmstorf (2021). Current atlantic meridional overturning circulation weakest in last millennium. Nature Geoscience 14(3), 118-120.

Caesar, L., S. Rahmstorf, A. Robinson, G. Feulner, and V. Saba (2018). Observed fingerprint of a weakening atlantic ocean overturning circulation. $\mathrm{Na}$ ture 556(7700), 191.

Cai, W. (2006). Antarctic ozone depletion causes an intensification of the southern ocean super-gyre circulation. Geophysical Research Letters 33(3).

Cai, W. and T. Cowan (2007). Trends in Southern Hemisphere circulation in IPCC AR4 models over 1950-99: Ozone depletion versus greenhouse forcing. Journal of Climate 20(4), 681-693.

Cai, W., G. Shi, T. Cowan, D. Bi, and J. Ribbe (2005). The response of the Southern Annular Mode, the East Australian Current, and the southern mid-latitude ocean circulation to global warming. Geophysical Research Letters 32(L23706), 1-4.

Callies, J. and R. Ferrari (2018). Dynamics of an abyssal circulation driven by bottom-intensified mixing on slopes. Journal of Physical Oceanography 48(6), 1257-1282.

Cessi, P. (2019). The global overturning circulation. Annual review of marine science 11, 249-270. 
Chang, P., R. Zhang, W. Hazeleger, C. Wen, X. Q. Wan, L. Ji, R. J. Haarsma, W. P. Breugem, and H. Seidel (2008). Oceanic link between abrupt changes in the North Atlantic Ocean and the African monsoon. Nature Geoscience 1(7), 444-448.

Chen, Z. and L. Wu (2012, jun). Long-term change of the Pacific North Equatorial Current bifurcation in SODA. Journal of Geophysical Research 117, C06016.

Cheng, W., J. C. Chiang, and D. Zhang (2013). Atlantic meridional overturning circulation (amoc) in cmip5 models: Rcp and historical simulations. Journal of Climate 26(18), 7187-7197.

Cheung, A. H., M. E. Mann, B. A. Steinman, L. M. Frankcombe, M. H. England, and S. K. Miller (2017). Comparison of low-frequency internal climate variability in cmip5 models and observations. Journal of Climate 30(12), 4763-4776.

Ciais, P., C. Sabine, G. Bala, L. Bopp, V. Brovkin, J. Canadell, A. Chhabra, R. DeFries, J. Galloway, M. Heimann, et al. (2014). Carbon and other biogeochemical cycles. In Climate Change 2013: The Physical Science Basis. Contribution of Working Group I to the Fifth Assessment Report of the Intergovernmental Panel on Climate Change, pp. 465-570. Cambridge University Press.

Cirano, M., M. M. Mata, E. Campos, and N. Deiro (2006). a Circulação Oceânica De Larga-Escala Na Região Oeste Do Atlântico Sul Com Base No Modelo De Circulação Global - OCCAM. Revista Brasileira de Geofisica 24, 209-230.

Coats, S. and J. E. Smerdon (2017). Climate variability: The atlantic's internal drum beat. Nature Geoscience 10(7), 470.

Collins, M., R. Knutti, J. Arblaster, J.-L. Dufresne, T. Fichefet, P. Friedlingstein, X. Gao, W. J. Gutowski, T. Johns, G. Krinner, et al. (2013). Long-term climate change: projections, commitments and irreversibility. In Climate Change 2013-The Physical Science Basis: Contribution of Working Group I to the Fifth Assessment Report of the Intergovernmental Panel on Climate Change, pp. 1029-1136. Cambridge University Press. 
Coman, M. A., R. W. Griffiths, and G. O. Hughes (2006). Sandström's experiments revisited. Journal of Marine Research 64(6), 783-796.

Combes, V. and R. P. Matano (2014). Trends in the Brazil/Malvinas Confluence region. Geophysical Research Letters 41(24), 8971-8977.

Crowley, T. J. (2000). Causes of climate change over the past 1000 years. Science 289(5477), 270-277.

Cunningham, S., S. Alderson, B. King, and M. Brandon (2003). Transport and variability of the antarctic circumpolar current in drake passage. Journal of Geophysical Research: Oceans 108(C5).

Cunningham, S. A. and R. Marsh (2010). Observing and modeling changes in the atlantic moc. Wiley Interdisciplinary Reviews: Climate Change 1(2), 180-191.

Danabasoglu, G., S. C. Bates, B. P. Briegleb, S. R. Jayne, M. Jochum, W. G. Large, S. Peacock, and S. G. Yeager (2012). The ccsm4 ocean component. Journal of Climate 25(5), 1361-1389.

Danabasoglu, G. and J. Marshall (2007). Effects of vertical variations of thickness diffusivity in an ocean general circulation model. Ocean Modelling 18(2), 122141.

de Ruijter, W. d., A. Biastoch, S. Drijfhout, J. Lutjeharms, R. Matano, T. Pichevin, P. Van Leeuwen, and W. Weijer (1999). Indian-atlantic interocean exchange: Dynamics, estimation and impact. Journal of Geophysical Research: Oceans 104(C9), 20885-20910.

Deser, C., A. S. Phillips, M. A. Alexander, and B. V. Smoliak (2014). Projecting north american climate over the next 50 years: Uncertainty due to internal variability. Journal of Climate 27(6), 2271-2296.

Deser, C., A. S. Phillips, R. A. Tomas, Y. M. Okumura, M. A. Alexander, A. Capotondi, J. D. Scott, Y.-O. Kwon, and M. Ohba (2012). Enso and pacific decadal variability in the community climate system model version 4 . Journal of Climate 25(8), 2622-2651. 
Diaz, H. F., R. Trigo, M. K. Hughes, M. E. Mann, E. Xoplaki, and D. Barriopedro (2011). Spatial and temporal characteristics of climate in medieval times revisited. Bulletin of the American Meteorological Society 92(11), 1487-1500.

Dong, S., S. Garzoli, M. Baringer, C. Meinen, and G. Goni (2009). Interannual variations in the atlantic meridional overturning circulation and its relationship with the net northward heat transport in the south atlantic. Geophysical Research Letters 36(20).

Donners, J. and S. Drijfhout (2004). The lagrangian view of south atlantic interocean exchange in a global ocean model compared with inverse model results. Journal of physical oceanography 34(5), 1019-1035.

Donohue, K., K. Tracey, D. Watts, M. P. Chidichimo, and T. Chereskin (2016). Mean antarctic circumpolar current transport measured in drake passage. Geophysical Research Letters 43(22), 11-760.

Drijfhout, S., G. J. Van Oldenborgh, and A. Cimatoribus (2012). Is a decline of amoc causing the warming hole above the north atlantic in observed and modeled warming patterns? Journal of Climate 25(24), 8373-8379.

Drijfhout, S. S., D. P. Marshall, and H. A. Dijkstra (2013). Conceptual models of the wind-driven and thermohaline circulation. In International Geophysics, Volume 103, pp. 257-282. Elsevier.

Drouin, K. L. and M. S. Lozier (2019). The surface pathways of the south atlantic: Revisiting the cold and warm water routes using observational data. Journal of Geophysical Research: Oceans 124(10), 7082-7103.

Drouin, K. L., M. S. Lozier, and W. E. Johns (2021). Variability and trends of the south atlantic subtropical gyre. Journal of Geophysical Research: Oceans 126(1), e2020JC016405.

Durgadoo, J. V., B. R. Loveday, C. J. Reason, P. Penven, and A. Biastoch (2013). Agulhas leakage predominantly responds to the southern hemisphere westerlies. Journal of Physical Oceanography 43(10), 2113-2131. 
Durgadoo, J. V., S. Rühs, A. Biastoch, and C. W. Böning (2017). Indian ocean sources of agulhas leakage. Journal of Geophysical Research: Oceans 122(4), 3481-3499.

Evans, D. G., J. Toole, G. Forget, J. D. Zika, A. C. Naveira Garabato, A. G. Nurser, and L. Yu (2017). Recent wind-driven variability in atlantic water mass distribution and meridional overturning circulation. Journal of Physical Oceanography 47(3), 633-647.

Evans, D. G., J. D. Zika, A. C. Naveira Garabato, and A. G. Nurser (2018). The cold transit of southern ocean upwelling. Geophysical Research Letters 45(24), 13-386.

Evans, D. L. and S. S. Signorini (1985). Vertical structure of the Brazil Current. Nature 315, 48-50.

Farneti, R. and P. R. Gent (2011). The effects of the eddy-induced advection coefficient in a coarse-resolution coupled climate model. Ocean Modelling 39(12), 135-145.

Fernández-Donado, J., C. Raible, C. Ammann, D. Barriopedro, E. GarciaBustamante, J. Jungclaus, S. Lorenz, J. Luterbacher, S. Phipps, J. Servonnat, et al. (2013). Large-scale temperature response to external forcing in simulations and reconstructions of the last millennium. Climate of the Past 9(1), 393-421.

Ferreira, D., P. Cessi, H. K. Coxall, A. De Boer, H. A. Dijkstra, S. S. Drijfhout, T. Eldevik, N. Harnik, J. F. McManus, D. P. Marshall, et al. (2018). Atlantic-pacific asymmetry in deep water formation. Annual Review of Earth and Planetary Sciences.

Feulner, G., S. Rahmstorf, A. Levermann, and S. Volkwardt (2013). On the origin of the surface air temperature difference between the hemispheres in earth's present-day climate. Journal of climate 26(18), 7136-7150.

Figueiredo Prado, L., I. Wainer, and P. Leite da Silva Dias (2018). Tropical atlantic response to last millennium volcanic forcing. Atmosphere 9(11), 421. 
Folland, C. K., A. W. Colman, D. P. Rowell, and M. K. Davey (2001). Predictability of northeast brazil rainfall and real-time forecast skill, 1987-98. Journal of Climate 14(9), 1937-1958.

Fontela, M., M. I. García-Ibáñez, D. A. Hansell, H. Mercier, and F. F. Pérez (2016). Dissolved organic carbon in the north atlantic meridional overturning circulation. Scientific reports 6(1), 1-9.

Fox-Kemper, B., H. T. Hewitt, G. A. C. Xiao, S. S. Drijfhout, T. L. Edwards, N. R. Golledge, M. Hemer, R. E. Kopp, G. Krinner, A. Mix, D. Notz, S. Nowicki, I. S. Nurhati, L. Ruiz, J.-B. Sallée, A. B. A. Slangen, and Y. Yu (2021). Ocean, cryosphere and sea level change. In Climate Change 2021: The Physical Science Basis. Contribution of Working Group I to the Sixth Assessment Report of the Intergovernmental Panel on Climate Change. Cambridge University Press.

Friedlingstein, P., M. W. Jones, M. O'sullivan, R. M. Andrew, J. Hauck, G. P. Peters, W. Peters, J. Pongratz, S. Sitch, C. L. Quéré, et al. (2019). Global carbon budget 2019. Earth System Science Data 11(4), 1783-1838.

Frierson, D. M., Y.-T. Hwang, N. S. Fučkar, R. Seager, S. M. Kang, A. Donohoe, E. A. Maroon, X. Liu, and D. S. Battisti (2013). Contribution of ocean overturning circulation to tropical rainfall peak in the northern hemisphere. Nature Geoscience 6(11), 940-944.

Friocourt, Y., S. Drijfhout, B. Blanke, and S. Speich (2005). Water mass export from drake passage to the atlantic, indian, and pacific oceans: a lagrangian model analysis. Journal of physical oceanography 35(7), 1206-1222.

Ganachaud, A. (2003). Large-scale mass transports, water mass formation, and diffusivities estimated from World Ocean Circulation Experiment (WOCE) hydrographic data. Journal of Geophysical Research 108(C7), 6-1 -.

Garzoli, S. and R. Matano (2011). The South Atlantic and the Atlantic meridional overturning circulation. Deep Sea Res. Part II Top. Stud. Oceanogr. 58(17), 1837-1847. 
Garzoli, S. L., M. O. Baringer, S. Dong, R. C. Perez, and Q. Yao (2013). South atlantic meridional fluxes. Deep Sea Research Part I: Oceanographic Research Papers 71, 21-32.

Garzoli, S. L., S. Dong, R. Fine, C. S. Meinen, R. C. Perez, C. Schmid, E. Van Sebille, and Q. Yao (2015). The fate of the deep western boundary current in the south atlantic. Deep Sea Research Part I: Oceanographic Research Papers 103, 125-136.

Gent, P. R. (2016). Effects of southern hemisphere wind changes on the meridional overturning circulation in ocean models. Annual review of marine science 8 , 79-94.

Gent, P. R. and J. C. Mcwilliams (1990). Isopycnal mixing in ocean circulation models. Journal of Physical Oceanography 20(1), 150-155.

Gill, A. E. (2016). Atmosphere—ocean dynamics. Elsevier.

Goddard, P. B., J. Yin, S. M. Griffies, and S. Zhang (2015). An extreme event of sea-level rise along the northeast coast of north america in 2009-2010. Nature Communications 6(1), 1-9.

Goes, M., L. N. Murphy, and A. C. Clement (2019). The stability of the amoc during heinrich events is not dependent on the amoc strength in an intermediate complexity earth system model ensemble. Paleoceanography and Paleoclimatology 34(8), 1359-1374.

Goni, G. J., F. Bringas, and P. N. DiNezio (2011, oct). Observed low frequency variability of the Brazil Current front. Journal of Geophysical Research 116, C10037.

Goni, G. J. and I. Wainer (2001). Investigation of the brazil current front variability from altimeter data. Journal of Geophysical Research: Oceans 106(C12), 31117-31128.

Gordon, A. L. (1985). Indian-atlantic transfer of thermocline water at the agulhas retroflection. Science 227(4690), 1030-1033. 
Gordon, A. L. (1986). Interocean exchange of thermocline water. Journal of Geophysical Research: Oceans 91(C4), 5037-5046.

Gordon, A. L. (1991). The role of thermohaline circulation in global climate change.

Gordon, A. L. (2001). Interocean exchange. In International Geophysics, Volume 77, pp. 303-314. Elsevier.

Goyal, R., A. Sen Gupta, M. Jucker, and M. H. England (2021). Historical and projected changes in the southern hemisphere surface westerlies. Geophysical Research Letters 48(4), e2020GL090849.

Graham, N., C. Ammann, D. Fleitmann, K. Cobb, and J. Luterbacher (2011). Support for global climate reorganization during the medieval climate anomaly. Climate Dynamics 37(5-6), 1217-1245.

Gray, L. J., J. Beer, M. Geller, J. D. Haigh, M. Lockwood, K. Matthes, U. Cubasch, D. Fleitmann, G. Harrison, L. Hood, et al. (2010). Solar influences on climate. Reviews of Geophysics 48(4).

Gruber, N., D. Clement, B. R. Carter, R. A. Feely, S. Van Heuven, M. Hoppema, M. Ishii, R. M. Key, A. Kozyr, S. K. Lauvset, et al. (2019). The oceanic sink for anthropogenic co2 from 1994 to 2007. Science 363(6432), 1193-1199.

Gulev, S. K., P. W. Thorne, J. Ahn, F. J. Dentener, C. M. Domingues, S. Gerland, D. Gong, D. S. Kaufman, H. C. Nnamchi, J. Quaas, J. A. Rivera, S. Sathyendranath, S. L. Smith, B. Trewin, K. von Shuckmann, and R. S. Vose (2021). Changing state of the climate system. In Climate Change 2021: The Physical Science Basis. Contribution of Working Group I to the Sixth Assessment Report of the Intergovernmental Panel on Climate Change. Cambridge University Press.

Gupta, A. S., A. Stellema, G. M. Pontes, A. S. Taschetto, A. Vergés, and V. Rossi (2021). Future changes to the upper ocean western boundary currents across two generations of climate models. Scientific reports 11(1), 1-12.

Häkkinen, S., P. B. Rhines, and D. L. Worthen (2015). Heat content variability in the north atlantic ocean in ocean reanalyses. Geophysical research letters 42(8), 2901-2909. 
Harper, S. (2000). Thermocline ventilation and pathways of tropical-subtropical water mass exchange. Tellus A 52(3), 330-345.

Hassan, T., R. J. Allen, W. Liu, and C. A. Randles (2021). Anthropogenic aerosol forcing of the atlantic meridional overturning circulation and the associated mechanisms in cmip6 models. Atmospheric Chemistry and Physics 21(8), 5821-5846.

Hastenrath, S. and L. Greischar (1993). Circulation mechanisms related to northeast brazil rainfall anomalies. Journal of Geophysical Research: Atmospheres 98(D3), 5093-5102.

Haug, G. H., K. A. Hughen, D. M. Sigman, L. C. Peterson, and U. Röhl (2001). Southward migration of the intertropical convergence zone through the holocene. Science 293(5533), 1304-1308.

Heinze, C., T. Blenckner, H. Martins, D. Rusiecka, R. Döscher, M. Gehlen, N. Gruber, E. Holland, Ø. Hov, F. Joos, et al. (2021). The quiet crossing of ocean tipping points. Proceedings of the National Academy of Sciences 118(9).

Hirschi, J. J.-M., B. Barnier, C. Böning, A. Biastoch, A. T. Blaker, A. Coward, S. Danilov, S. Drijfhout, K. Getzlaff, S. M. Griffies, et al. (2020). The atlantic meridional overturning circulation in high-resolution models. Journal of Geophysical Research: Oceans 125(4), e2019JC015522.

Hu, D., L. Wu, W. Cai, A. S. Gupta, A. Ganachaud, B. Qiu, A. L. Gordon, X. Lin, Z. Chen, S. Hu, G. Wang, Q. Wang, J. Sprintall, T. Qu, Y. Kashino, F. Wang, and W. S. Kessler (2015). Pacific western boundary currents and their roles in climate. Nature 522(7556), 299-308.

Huang, W., S. Feng, C. Liu, J. Chen, J. Chen, and F. Chen (2018). Changes of climate regimes during the last millennium and the twenty-first century simulated by the community earth system model. Quaternary Science Reviews 180, 42-56.

Hummels, R., P. Brandt, M. Dengler, J. Fischer, M. Araujo, D. Veleda, and J. V. Durgadoo (2015). Interannual to decadal changes in the western boundary 
circulation in the atlantic at 11 s. Geophysical Research Letters 42(18), 76157622.

Hunke, E. C., W. H. Lipscomb, A. K. Turner, N. Jeffery, and S. Elliott (2015). CICE : the Los Alamos Sea Ice Model Documentation and Software User's Manual LA-CC-06-012. pp. 115.

Hurrell, J. W., M. M. Holland, P. R. Gent, S. Ghan, J. E. Kay, P. J. Kushner, J.-F. Lamarque, W. G. Large, D. Lawrence, K. Lindsay, et al. (2013). The community earth system model: a framework for collaborative research. Bulletin of the American Meteorological Society 94(9), 1339-1360.

Jackson, L., C. Dubois, G. Forget, K. Haines, M. Harrison, D. Iovino, A. Köhl, D. Mignac, S. Masina, K. Peterson, et al. (2019). The mean state and variability of the north atlantic circulation: A perspective from ocean reanalyses. Journal of Geophysical Research: Oceans 124(12), 9141-9170.

Jackson, L. and R. Wood (2018). Hysteresis and resilience of the amoc in an eddy-permitting gcm. Geophysical Research Letters 45(16), 8547-8556.

Jackson, L. C., K. A. Peterson, C. D. Roberts, and R. A. Wood (2016). Recent slowing of atlantic overturning circulation as a recovery from earlier strengthening. Nature Geoscience 9(7), 518-522.

Jamet, Q., W. K. Dewar, N. Wienders, B. Deremble, S. Close, and T. Penduff (2020). Locally and remotely forced subtropical amoc variability: a matter of time scales. Journal of Climate 33(12), 5155-5172.

Johns, W. E., M. O. Baringer, L. Beal, S. Cunningham, T. Kanzow, H. L. Bryden, J. Hirschi, J. Marotzke, C. Meinen, B. Shaw, et al. (2011). Continuous, array-based estimates of atlantic ocean heat transport at $26.5 \mathrm{n}$. Journal of Climate 24(10), 2429-2449.

Johnson, H. L., P. Cessi, D. P. Marshall, F. Schloesser, and M. A. Spall (2019). Recent contributions of theory to our understanding of the atlantic meridional overturning circulation. Journal of Geophysical Research: Oceans 124(8), 5376-5399. 
Jones, C. S. and P. Cessi (2016). Interbasin transport of the meridional overturning circulation. Journal of Physical Oceanography 46(4), 1157-1169.

Jones, P. D. and M. E. Mann (2004). Climate over past millennia. Reviews of Geophysics 42(2).

Juckes, M. N., M. R. Allen, K. R. Briffa, J. Esper, G. Hegerl, A. Moberg, T. Osborn, and S. Weber (2007). Millennial temperature reconstruction intercomparison and evaluation. Climate of the Past 3(4), 591-609.

Kang, S. M., I. M. Held, D. M. Frierson, and M. Zhao (2008). The response of the itcz to extratropical thermal forcing: Idealized slab-ocean experiments with a gcm. Journal of Climate 21(14), 3521-3532.

Kang, S. M., R. Seager, D. M. Frierson, and X. Liu (2015). Croll revisited: Why is the northern hemisphere warmer than the southern hemisphere? Climate Dynamics 44(5), 1457-1472.

Kaufman, D. S., D. P. Schneider, N. P. McKay, C. M. Ammann, R. S. Bradley, K. R. Briffa, G. H. Miller, B. L. Otto-Bliesner, J. T. Overpeck, B. M. Vinther, et al. (2009). Recent warming reverses long-term arctic cooling. Science 325(5945), 1236-1239.

Kay, J., C. Deser, A. Phillips, A. Mai, C. Hannay, G. Strand, J. Arblaster, S. Bates, G. Danabasoglu, J. Edwards, et al. (2015). The community earth system model (cesm) large ensemble project: A community resource for studying climate change in the presence of internal climate variability. Bulletin of the American Meteorological Society 96(8), 1333-1349.

Keigwin, L. and E. Boyle (2000). Detecting holocene changes in thermohaline circulation. Proceedings of the National Academy of Sciences 97(4), 13431346.

Kendall, M. G. (1975). Rank Correlation Methods (4 ed.). London.

Kersale, M., C. S. Meinen, R. C. Perez, M. Le Henaff, D. Valla, T. Lamont, O. Sato, S. Dong, T. Terre, M. van Caspel, et al. (2020). Highly variable upper and abyssal overturning cells in the south atlantic. Science advances 6(32), eaba7573. 
Kilbourne, H., M. Klockmann, E. Moreno-Chamarro, P. Ortega, A. Romanou, M. Srokosz, Z. Szuts, K. Thirumalai, I. Hall, P. Heimbach, et al. (2016). Connecting paleo and modern oceanographic data to understand atlantic meridional overturning circulation over decades to centuries. A US CLIVAR Workshop Report Report 2017-3, 26pp.

Knight, J. R., C. K. Folland, and A. A. Scaife (2006). Climate impacts of the atlantic multidecadal oscillation. Geophysical Research Letters 33(17).

Kostov, Y., K. C. Armour, and J. Marshall (2014). Impact of the atlantic meridional overturning circulation on ocean heat storage and transient climate change. Geophysical Research Letters 41(6), 2108-2116.

Kuhlbrodt, T. (2008). On sandström's inferences from his tank experiments: a hundred years later. Tellus A: Dynamic Meteorology and Oceanography 60(5), 819-836.

Kuhlbrodt, T., A. Griesel, M. Montoya, A. Levermann, M. Hofmann, and S. Rahmstorf (2007). On the driving processes of the atlantic meridional overturning circulation. Reviews of Geophysics 45(2).

Kump, L., J. Kasting, and R. Crane (2004). The Earth System. Pearson Prentice Hall.

Kushner, P. J., I. M. Held, and T. L. Delworth (2001). Southern hemisphere atmospheric circulation response to global warming. Journal of Climate 14(10), 2238-2249.

Landrum, L., B. L. Otto-Bliesner, E. R. Wahl, A. Conley, P. J. Lawrence, N. Rosenbloom, and H. Teng (2013). Last millennium climate and its variability in ccsm4. Journal of climate 26(4), 1085-1111.

Large, W. and S. Yeager (2009). The global climatology of an interannually varying air-sea flux data set. Climate dynamics 33(2-3), 341-364.

Lawrence, D. M., K. W. Oleson, M. G. Flanner, P. E. Thornton, S. C. Swenson, J. Peter, X. Zeng, Z.-I. Yang, S. Levis, K. Sakaguchi, G. B. Bonan, and A. G. 
Slater (2009). Parameterization Improvements and Functional and Structural Advances in Version 4 of the Community Land Model. 3, 1-27.

Le, T., J. Sjolte, and R. Muscheler (2016). The influence of external forcing on subdecadal variability of regional surface temperature in cmip5 simulations of the last millennium. Journal of Geophysical Research: Atmospheres 121(4), 1671-1682.

Lenton, T. M., H. Held, E. Kriegler, J. W. Hall, W. Lucht, S. Rahmstorf, and H. J. Schellnhuber (2008). Tipping elements in the earth's climate system. Proceedings of the national Academy of Sciences 105(6), 1786-1793.

Leroux, S., T. Penduff, L. Bessières, J.-M. Molines, J.-M. Brankart, G. Sérazin, B. Barnier, and L. Terray (2018). Intrinsic and atmospherically forced variability of the amoc: Insights from a large-ensemble ocean hindcast. Journal of Climate 31(3), 1183-1203.

Li, H., A. Fedorov, and W. Liu (2021). Amoc stability and diverging response to arctic sea ice decline in two climate models. Journal of Climate 34(13), 5443-5460.

Li, W., L. Li, M. Ting, Y. Deng, Y. Kushnir, Y. Liu, Y. Lu, C. Wang, and P. Zhang (2013). Intensification of the Southern Hemisphere summertime subtropical anticyclones in a warming climate. Geophysical Research Letters 40(22), 59595964.

Li, W., L. Li, M. Ting, and Y. Liu (2012). Intensification of Northern Hemisphere subtropical highs in a warming climate. Nature Geoscience 5(11), 830-834.

Liu, W., S.-P. Xie, Z. Liu, and J. Zhu (2017). Overlooked possibility of a collapsed atlantic meridional overturning circulation in warming climate. Science Advances 3(1), e1601666.

Ljungqvist, F. et al. (2010). A new reconstruction of temperature variability in the extra-tropical northern hemisphere during the last two millennia. Geogr. Ann. A 92(3), 339-351. 
Ljungqvist, F. C., Q. Zhang, G. Brattström, P. J. Krusic, A. Seim, Q. Li, Q. Zhang, and A. Moberg (2019). Centennial-scale temperature change in last millennium simulations and proxy-based reconstructions. Journal of Climate 32(9), 24412482.

Lohmann, J. and P. D. Ditlevsen (2021). Risk of tipping the overturning circulation due to increasing rates of ice melt. Proceedings of the National Academy of Sciences 118(9).

Lozier, M. S. (2010). Deconstructing the conveyor belt. science 328(5985), 1507-1511.

Lozier, M. S. (2012). Overturning in the north atlantic. Annual review of marine science 4, 291-315.

Lozier, M. S., S. Bacon, A. S. Bower, S. A. Cunningham, M. F. De Jong, L. De Steur, B. Deyoung, J. Fischer, S. F. Gary, B. J. Greenan, et al. (2017). Overturning in the subpolar north atlantic program: A new international ocean observing system. Bulletin of the American Meteorological Society 98(4), 737-752.

Lozier, M. S., S. F. Gary, and A. S. Bower (2013). Simulated pathways of the overflow waters in the north atlantic: Subpolar to subtropical export. Deep Sea Research Part II: Topical Studies in Oceanography 85, 147-153.

Lübbecke, J. F., N. J. Burls, C. J. C. Reason, and M. J. McPhaden (2014). Variability in the South Atlantic Anticyclone and the Atlantic Niño Mode*. Journal of Climate 27(21), 8135-8150.

Luko, C., I. da Silveira, I. Simoes-Sousa, J. Araujo, and A. Tandon (2021). Revisiting the atlantic south equatorial current. Journal of Geophysical Research: Oceans 126(7), e2021JC017387.

Lumpkin, R. and S. Garzoli (2011, jan). Interannual to decadal changes in the western South Atlantic's surface circulation. Journal of Geophysical Research 116, $1-10$.

Lumpkin, R. and K. Speer (2003). Large-Scale Vertical and Horizontal Circulation in the North Atlantic Ocean. Journal of Physical Oceanography 33, 1902-1920. 
Lumpkin, R. and K. Speer (2007). Global ocean meridional overturning. Journal of Physical Oceanography 37(10), 2550-2562.

Lund, D. C. and W. Curry (2006). Florida current surface temperature and salinity variability during the last millennium. Paleoceanography 21(2).

Lund, D. C., J. Lynch-Stieglitz, and W. B. Curry (2006). Gulf stream density structure and transport during the past millennium. Nature 444(7119), 601.

Ma, J., M. Feng, J. Lan, and D. Hu (2020). Projected future changes of meridional heat transport and heat balance of the indian ocean. Geophysical Research Letters 47(4), e2019GL086803.

Macdonald, A. M. (1998). The global ocean circulation: A hydrographic estimate and regional analysis. Progress in Oceanography 41(3), 281-382.

Mahajan, S., R. Zhang, T. L. Delworth, S. Zhang, A. J. Rosati, and Y.-S. Chang (2011). Predicting atlantic meridional overturning circulation (amoc) variations using subsurface and surface fingerprints. Deep Sea Research Part II: Topical Studies in Oceanography 58(17-18), 1895-1903.

Maher, N., F. Lehner, and J. Marotzke (2020). Quantifying the role of internal variability in the temperature we expect to observe in the coming decades. Environmental Research Letters 15(5), 054014.

Malanotte-Rizzoli, P., K. Hedstromb, H. Arango, and D. B. Haidvogel (2000). Water mass pathways between the subtropical and tropical ocean in a climatological simulation of the North Atlantic ocean circulation. Dynamics of Atmospheres and Oceans 32, 331-371.

Mankin, J. S., F. Lehner, S. Coats, and K. A. McKinnon (2020). The value of initial condition large ensembles to robust adaptation decision-making. Earth's Future 8(10), e2012EF001610.

Mann, H. B. (1945). Non-parametric tests against trend. Econometrica 33, 245259. 
Mann, M. E., Z. Z., R. S., B. R. S., H. M. K., S. D., A. C., F. G., and N. F (2009). Global signatures and dynamical origins of the little ice age and medieval climate anomaly. Science 326, 1256.

Mann, M. E., Z. Zhang, M. K. Hughes, R. S. Bradley, S. K. Miller, S. Rutherford, and F. Ni (2008). Proxy-based reconstructions of hemispheric and global surface temperature variations over the past two millennia. Proceedings of the National Academy of Sciences 105(36), 13252-13257.

Marcello, F., I. Wainer, P. R. Gent, B. L. Otto-Bliesner, and E. C. Brady (2019). South Atlantic Surface Boundary Current System during the Last Millennium in the CESM-LME: The Medieval Climate Anomaly and Little Ice Age. Geosciences 9(7), 299.

Marcello, F., I. Wainer, and R. R. Rodrigues (2018). South Atlantic Subtropical Gyre late twentieth century changes. Journal of Geophysical Research: Oceans 123(8), 5194-5209.

Marsh, R., W. Hazeleger, A. Yool, and E. J. Rohling (2007). Stability of the thermohaline circulation under millennial co2 forcing and two alternative controls on atlantic salinity. Geophysical Research Letters 34(3).

Marshall, G. J. (2003). Trends in the Southern Annular Mode from observations and reanalyses. Journal of Climate 16(24), 4134-4143.

Marshall, J., K. C. Armour, J. R. Scott, Y. Kostov, U. Hausmann, D. Ferreira, T. G. Shepherd, and C. M. Bitz (2014). The ocean's role in polar climate change: asymmetric arctic and antarctic responses to greenhouse gas and ozone forcing. Philosophical Transactions of the Royal Society A: Mathematical, Physical and Engineering Sciences 372(2019), 20130040.

Marshall, J., A. Donohoe, D. Ferreira, and D. McGee (2014). The ocean's role in setting the mean position of the inter-tropical convergence zone. Climate Dynamics 42(7), 1967-1979.

Marshall, J. and R. A. Plumb (2016). Atmosphere, ocean and climate dynamics: an introductory text. Academic Press. 
Marshall, J. and K. Speer (2012). Closure of the meridional overturning circulation through southern ocean upwelling. Nature Geoscience 5(3), 171-180.

Martínez-Moreno, J., A. M. Hogg, M. H. England, N. C. Constantinou, A. E. Kiss, and A. K. Morrison (2021). Global changes in oceanic mesoscale currents over the satellite altimetry record. Nature Climate Change 11(5), 397-403.

Masson-Delmotte, V., P. Zhai, A. Pirani, S. L. Connors, C. Péan, S. Berger, N. Caud, Y. Chen, L. Goldfarb, M. I. Gomis, M. Huang, K. Leitzell, E. Lonnoy, J. B. R. Matthews, T. K. Maycock, T. Waterfield, O. Yelekçi, R. Yu, and B. Zhou (2021). Climate Change 2021: The Physical Science Basis. Contribution of Working Group I to the Sixth Assessment Report of the Intergovernmental Panel on Climate Change. Cambridge University Press. In Press.

MassonDelmotte, V., P. Zhai, A. Pirani, S. Connors, C. Péan, S. Berger, N. Caud, Y. Chen, L. Goldfarb, M. Gomis, M. Huang, K. Leitzell, E. Lonnoy, J. Matthews, T. Maycock, T. Waterfield, O. Yelekçi, R. Yu, and B. Zhou (2021). Summary for policymakers. In Climate Change 2021: The Physical Science Basis. Contribution of Working Group I to the Sixth Assessment Report of the Intergovernmental Panel on Climate Change. In Press. Cambridge University Press.

McCarthy, G. D., D. A. Smeed, W. E. Johns, E. Frajka-Williams, B. I. Moat, D. Rayner, M. O. Baringer, C. S. Meinen, J. Collins, and H. L. Bryden (2015). Measuring the Atlantic Meridional Overturning Circulation at 26N. Progress in Oceanography 130, 91-111.

McCreary, J. P. and P. Lu (1994). Interaction between the Subtropical and Equatorial Ocean Circulations: The Subtropical Cell. Journal of Physical Oceanography 24(2), 466-497.

Meehl, G. A., J. M. Arblaster, G. Branstator, and H. Van Loon (2008). A coupled air-sea response mechanism to solar forcing in the pacific region. Journal of Climate 21(12), 2883-2897.

Meehl, G. A., J. M. Arblaster, K. Matthes, F. Sassi, and H. van Loon (2009). Amplifying the pacific climate system response to a small 11-year solar cycle forcing. Science 325(5944), 1114-1118. 
Meinen, C. S., S. Speich, A. R. Piola, I. Ansorge, E. Campos, M. Kersalé, T. Terre, M. P. Chidichimo, T. Lamont, O. T. Sato, et al. (2018). Meridional overturning circulation transport variability at 34.5 s during 2009-2017: Baroclinic and barotropic flows and the dueling influence of the boundaries. Geophysical Research Letters 45(9), 4180-4188.

Menary, M. B., J. Robson, R. P. Allan, B. B. Booth, C. Cassou, G. Gastineau, J. Gregory, D. Hodson, C. Jones, J. Mignot, et al. (2020). Aerosol-forced amoc changes in cmip6 historical simulations. Geophysical research letters 47(14), e2020GL088166.

Meredith, M. P., P. L. Woodworth, T. K. Chereskin, D. P. Marshall, L. C. Allison, G. R. Bigg, K. Donohue, K. J. Heywood, C. W. Hughes, A. Hibbert, et al. (2011). Sustained monitoring of the southern ocean at drake passage: Past achievements and future priorities. Reviews of Geophysics 49(4).

Miller, G. H., Á. Geirsdóttir, Y. Zhong, D. J. Larsen, B. L. Otto-Bliesner, M. M. Holland, D. A. Bailey, K. A. Refsnider, S. J. Lehman, J. R. Southon, et al. (2012). Abrupt onset of the little ice age triggered by volcanism and sustained by sea-ice/ocean feedbacks. Geophysical Research Letters 39(2).

Moat, B. I., D. A. Smeed, E. Frajka-Williams, D. G. Desbruyères, C. Beaulieu, W. E. Johns, D. Rayner, A. Sanchez-Franks, M. O. Baringer, D. Volkov, et al. (2020). Pending recovery in the strength of the meridional overturning circulation at $26^{\circ}$ n. Ocean Science 16(4), 863-874.

Moffa-Sánchez, P., E. Moreno-Chamarro, D. Reynolds, P. Ortega, L. Cunningham, D. Swingedouw, D. E. Amrhein, J. Halfar, L. Jonkers, J. H. Jungclaus, et al. (2019). Variability in the northern north atlantic and arctic oceans across the last two millennia: A review. Paleoceanography and Paleoclimatology 34(8), 1399-1436.

Molinari, R. L. (1982). Observations of eastward currents in the tropical south atlantic ocean: 1978-1980. Journal of Geophysical Research: Oceans 87(C12), 9707-9714. 
Moreno-Chamarro, E., D. Zanchettin, K. Lohmann, J. Luterbacher, and J. H. Jungclaus (2017). Winter amplification of the european little ice age cooling by the subpolar gyre. Scientific reports 7(1), 9981.

Morrison, A. K., S. M. Griffies, M. Winton, W. G. Anderson, and J. L. Sarmiento (2016). Mechanisms of southern ocean heat uptake and transport in a global eddying climate model. Journal of Climate 29(6), 2059-2075.

Munk, W. H. (1950). On the wind-driven ocean circulation. Journal of Atmospheric Sciences 7(2), 80-93.

Munoz, S. E. and S. G. Dee (2017). El niño increases the risk of lower mississippi river flooding. Scientific reports $7(1), 1-7$.

Neale, R. B., C.-c. Chen, P. H. Lauritzen, D. L. Williamson, A. J. Conley, A. K. Smith, M. Mills, and H. Morrison (2010). Description of the NCAR Community Atmosphere Model ( CAM 5 . 0 ). Technical Report April.

Neukom, R., J. Gergis, D. J. Karoly, H. Wanner, M. Curran, J. Elbert, F. GonzálezRouco, B. K. Linsley, A. D. Moy, I. Mundo, et al. (2014). Inter-hemispheric temperature variability over the past millennium. Nature Climate Change 4(5), 362.

Neukom, R., J. Luterbacher, R. Villalba, M. Küttel, D. Frank, P. Jones, M. Grosjean, H. Wanner, J.-C. Aravena, D. Black, et al. (2011). Multiproxy summer and winter surface air temperature field reconstructions for southern south america covering the past centuries. Climate Dynamics 37(1-2), 35-51.

Neukom, R., N. Steiger, J. J. Gómez-Navarro, J. Wang, and J. P. Werner (2019). No evidence for globally coherent warm and cold periods over the preindustrial common era. Nature 571(7766), 550-554.

Oliver, E. and N. Holbrook (2014). Extending our understanding of south pacific gyre "spin-up": Modeling the east australian current in a future climate. Journal of Geophysical Research: Oceans 119(5), 2788-2805. 
Orsi, A. H., T. Whitworth III, and W. D. Nowlin Jr (1995). On the meridional extent and fronts of the antarctic circumpolar current. Deep Sea Research Part I: Oceanographic Research Papers 42(5), 641-673.

Otto-Bliesner, B. L., E. C. Brady, J. Fasullo, A. Jahn, L. Landrum, S. Stevenson, N. Rosenbloom, A. Mai, and G. Strand (2015). Climate variability and change since 850 C.E.: An ensemble approach with the Community Earth System Model (CESM). Bulletin of the American Meteorological Society 97(5), 787-801.

PAGES 2k Consortium (2013). Continental-scale temperature variability during the past two millennia. Nature geoscience 6(5), 339.

PAGES 2k-PMIP3 Group (2015). Continental-scale temperature variability in pmip3 simulations and pages $2 \mathrm{k}$ regional temperature reconstructions over the past millennium. Climate of the Past 11(12), 1673-1699.

Palter, J. B. (2015). The role of the gulf stream in european climate. Annual review of marine science $7,113-137$.

Palter, J. B. and M. S. Lozier (2008). On the source of gulf stream nutrients. Journal of Geophysical Research: Oceans 113(C6).

Pedlosky, J. (1996). Ocean circulation theory. Springer Science \& Business Media.

Pereira, J., M. Gabioux, M. Marta-almeida, M. Cirano, A. M. Paiva, and A. L. Aguiar (2014). The Bifurcation of the Western Boundary Current System of the South Atlantic Ocean. Brazilian Journal of Geophysics 32, 241-257.

Pérez, F. F., H. Mercier, M. Vázquez-Rodríguez, P. Lherminier, A. Velo, P. C. Pardo, G. Rosón, and A. F. Ríos (2013). Atlantic ocean co 2 uptake reduced by weakening of the meridional overturning circulation. Nature Geoscience 6(2), $146-152$.

Peterson, R. G. and L. Stramma (1991). Upper-level circulation in the south atlantic ocean. Progress in oceanography 26(1), 1-73.

Polvani, L. M., D. W. Waugh, G. J. P. Correa, and S.-W. Son (2011). Stratospheric Ozone Depletion: The Main Driver of Twentieth-Century Atmospheric Circulation Changes in the Southern Hemisphere. Journal of Climate 24(3), 795-812. 
Pontes, G. M., A. S. Gupta, and A. S. Taschetto (2016). Projected changes to South Atlantic boundary currents and confluence region in the CMIP5 models: the role of wind and deep ocean changes. Environmental Research Letters 11(9), 1-9.

Pörtner, H.-O., D. C. Roberts, V. Masson-Delmotte, P. Zhai, M. Tignor, E. Poloczanska, K. Mintenbeck, M. Nicolai, A. Okem, J. Petzold, et al. (2019). Ipcc special report on the ocean and cryosphere in a changing climate. IPCC Intergovernmental Panel on Climate Change: Geneva, Switzerland 1(3).

Qu, T., I. Fukumori, and R. A. Fine (2019). Spin-up of the southern hemisphere super gyre. Journal of Geophysical Research: Oceans 124(1), 154-170.

Rahmstorf, S. (1996). On the freshwater forcing and transport of the atlantic thermohaline circulation. Climate Dynamics 12(12), 799-811.

Rahmstorf, S. (2000). The thermohaline ocean circulation: A system with dangerous thresholds? Climatic Change 46(3), 247.

Rahmstorf, S. (2002). Ocean circulation and climate during the past 120,000 years. Nature 419(6903), 207-214.

Rahmstorf, S. (2006). Thermohaline ocean circulation. Encyclopedia of quaternary sciences 5 .

Rahmstorf, S., J. E. Box, G. Feulner, M. E. Mann, A. Robinson, S. Rutherford, and E. J. Schaffernicht (2015). Exceptional twentieth-century slowdown in atlantic ocean overturning circulation. Nature climate change 5(5), 475-480.

Reid, J. L. (1994). On the total geostrophic circulation of th North Atlantic Ocean. Prog. Oceanogr. 33, 1-92.

Rhein, M., S. Rintoul, S. Aoki, E. Campos, D. Chambers, R. Feely, S. Gulev, G. Johnson, S. Josey, A. Kostianoy, et al. (2013). Observations: ocean. in 'climate change 2013: the physical science basis. contribution of working group $\mathrm{i}$ to the fifth assessment report of the intergovernmental panel on climate change'. Cambridge University Press, Cambridge, United Kingdom and New York, NY, USA. 
Richardson, P. L. (2007). Agulhas leakage into the Atlantic estimated with subsurface floats and surface drifters. Deep-Sea Research Part I: Oceanographic Research Papers 54(8), 1361-1389.

Richardson, P. L. (2008). On the history of meridional overturning circulation schematic diagrams. Progress in Oceanography 76(4), 466-486.

Ridgway, K. and J. Dunn (2007). Observational evidence for a southern hemisphere oceanic supergyre. Geophysical Research Letters 34(13).

Rintoul, S. R. (1991). South atlantic interbasin exchange. Journal of Geophysical Research: Oceans 96(C2), 2675-2692.

Rintoul, S. R. (2018). The global influence of localized dynamics in the southern ocean. Nature 558(7709), 209-218.

Rintoul, S. R., C. W. Hughes, and D. Olbers (2001). The antarctic circumpolar current system. In International Geophysics, Volume 77, pp. 271-XXXVI. Elsevier.

Ritchie, P. D., J. J. Clarke, P. M. Cox, and C. Huntingford (2021). Overshooting tipping point thresholds in a changing climate. Nature 592(7855), 517-523.

Roberts, C., L. Jackson, and D. McNeall (2014). Is the 2004-2012 reduction of the atlantic meridional overturning circulation significant? Geophysical Research Letters 41(9), 3204-3210.

Rodgers, J. L. and W. A. Nicewander (1988). Thirteen Ways to Look at the Correlation Coefficient. The American Statistician 42(1), 59-66.

Rodrigues, R. R., L. M. Rothstein, and M. Wimbush (2007, jan). Seasonal Variability of the South Equatorial Current Bifurcation in the Atlantic Ocean: A Numerical Study. Journal of Physical Oceanography 37(1), 16-30.

Rodrigues, R. R., M. Wimbush, D. R. Watts, L. M. Rothstein, and M. Ollitrault (2010). South atlantic mass transports obtained from subsurface float and hydrographic data. Journal of Marine Research 68(6), 819-850.

Roemmich, D. (2007). Super spin in the southern seas. Nature 449, 34-35. 
Roemmich, D., J. Gilson, R. Davis, P. Sutton, S. Wijffels, and S. Riser (2007, feb). Decadal Spinup of the South Pacific Subtropical Gyre. Journal of Physical Oceanography 37(2), 162-173.

Roemmich, D., J. Gilson, P. Sutton, and N. Zilberman (2016). Multidecadal change of the south pacific gyre circulation. Journal of Physical Oceanography 46(6), 1871-1883.

Rosón, G., A. F. Ríos, F. F. Pérez, A. Lavín, and H. L. Bryden (2003). Carbon distribution, fluxes, and budgets in the subtropical north atlantic ocean (24.5 n). Journal of Geophysical Research: Oceans 108(C5).

Rouault, M., P. Penven, and B. Pohl (2009). Warming in the agulhas current system since the 1980's. Geophysical Research Letters 36(12).

Rühs, S., J. V. Durgadoo, E. Behrens, and A. Biastoch (2013). Advective timescales and pathways of agulhas leakage. Geophysical Research Letters 40(15), 39974000 .

Rühs, S., F. U. Schwarzkopf, S. Speich, and A. Biastoch (2019). Cold vs. warm water route-sources for the upper limb of the atlantic meridional overturning circulation revisited in a high-resolution ocean model. Ocean Science 15(3), 489-512.

Saenko, O. a., J. C. Fyfe, and M. H. England (2005, jul). On the response of the oceanic wind-driven circulation to atmospheric $\mathrm{CO} 2$ increase. Climate Dynamics 25(4), 415-426.

Sandström, J. W. (1908). Dynamische versuche mit meerwasser.

Scheffer, M., S. Carpenter, J. A. Foley, C. Folke, and B. Walker (2001). Catastrophic shifts in ecosystems. Nature 413(6856), 591-596.

Schmid, C. (2014). Mean vertical and horizontal structure of the subtropical circulation in the south atlantic from three-dimensional observed velocity fields. Deep Sea Research Part I: Oceanographic Research Papers 91, 50-71. 
Schmid, C. and S. Majumder (2018). Transport variability of the brazil current from observations and a data assimilation model. Ocean Science 14(3), 417-436.

Schmidt, G. A., J. H. Jungclaus, C. Ammann, E. Bard, P. Braconnot, T. Crowley, G. Delaygue, F. Joos, N. Krivova, R. Muscheler, et al. (2011). Climate forcing reconstructions for use in pmip simulations of the last millennium ( $v 1.0)$. Geoscientific Model Development 4(1), 33-45.

Schmittner, A., J. C. Chiang, and S. R. Hemming (2007). Introduction: The ocean's meridional overturning circulation. Washington DC American Geophysical Union Geophysical Monograph Series 173, 1-4.

Schneider, W., M. Fukasawa, J. Garcés-Vargas, L. Bravo, H. Uchida, T. Kawano, and R. Fuenzalida (2007). Spin-up of south pacific subtropical gyre freshens and cools the upper layer of the eastern south pacific ocean. Geophysical Research Letters 34(24).

Schott, F. a., J. Fischer, and L. Stramma (1998). Transports and Pathways of the Upper-Layer Circulation in the Western Tropical Atlantic. Journal of Physical Oceanography 28(10), 1904-1928.

Schott, F. A., L. Stramma, and J. Fischer (1995). The warm water inflow into the western tropical atlantic boundary regime, spring 1994. Journal of Geophysical Research: Oceans 100(C12), 24745-24760.

Schott, G. (1902). Scientific results of the german deep-sea expedition on the steamer "valdivia" 1898-1899, volume i: Oceanography and maritime meteorology.

Schwalm, C. R., S. Glendon, and P. B. Duffy (2020). Rcp8. 5 tracks cumulative co2 emissions. Proceedings of the National Academy of Sciences 117(33), 19656-19657.

Seager, R. and I. R. Simpson (2016). Western boundary currents and climate change. Journal of Geophysical Research: Oceans 121(9), 7212-7214.

Sen Gupta, A., S. McGregor, E. Van Sebille, A. Ganachaud, J. N. Brown, and A. Santoso (2016). Future changes to the indonesian throughflow and pacific 
circulation: The differing role of wind and deep circulation changes. Geophysical Research Letters 43(4), 1669-1678.

Shi, J.-R., S.-P. Xie, and L. D. Talley (2018). Evolving relative importance of the southern ocean and north atlantic in anthropogenic ocean heat uptake. Journal of Climate 31(18), 7459-7479.

Shindell, D. T. and G. A. Schmidt (2004). Southern hemisphere climate response to ozone changes and greenhouse gas increases. Geophysical Research Letters 31(18).

Sloyan, B. M. and S. R. Rintoul (2001). The southern ocean limb of the global deep overturning circulation. Journal of Physical Oceanography 31(1), 143-173.

Smeed, D., S. Josey, C. Beaulieu, W. Johns, B. Moat, E. Frajka-Williams, D. Rayner, C. Meinen, M. Baringer, H. Bryden, et al. (2018). The North Atlantic Ocean is in a state of reduced overturning. Geophysical Research Letters 45(3), 1527-1533.

Smeed, D. A., G. D. McCarthy, S. A. Cunningham, E. Frajka-Williams, D. Rayner, W. Johns, C. S. Meinen, M. O. Baringer, B. I. Moat, A. Duchez, et al. (2014). Observed decline of the Atlantic meridional overturning circulation 2004-2012. Ocean Science 10(1), 29-38.

Smerdon, J. and H. Pollack (2016). Reconstructing earth's surface temperature over the past 2000 years: the science behind the headlines. Wiley Interdisciplinary Reviews: Climate Change.

Smith, R., P. Jones, B. Briegleb, F. Bryan, G. Danabasoglu, J. Dennis, J. Dukowicz, C. Eden, B. Fox-Kemper, P. Gent, M. Hecht, S. Jayne, M. Jochum, W. Large, K. Lindsay, M. Maltrud, N. Norton, S. Peacock, M. Vertenstein, and S. Yeager (2010). The Parallel Ocean Program (POP) reference manual: Ocean component of the Community Climate System Model (CCSM). Technical report.

Smith, S. W. (1997). The Scientist and Engineer's Guide to Digital Signal Processing (Second ed.). 
Solomon, S., M. Manning, M. Marquis, D. Qin, et al. (2007). Climate change 2007-the physical science basis: Working group I contribution to the fourth assessment report of the IPCC, Volume 4. Cambridge university press.

Son, S.-W., N. F. Tandon, L. M. Polvani, and D. W. Waugh (2009). Ozone hole and southern hemisphere climate change. Geophysical Research Letters 36(15).

Speich, S., B. Blanke, and W. Cai (2007). Atlantic meridional overturning circulation and the Southern Hemisphere supergyre. Geophysical Research Letters 34(23).

Speich, S., B. Blanke, and G. Madec (2001). Warm and cold water routes of an OGCM thermohaline conveyor belt. Geophysical research letters 28(2), 311-314.

Srokosz, M. and H. Bryden (2015). Observing the atlantic meridional overturning circulation yields a decade of inevitable surprises. Science 348(6241).

Srokosz, M., G. Danabasoglu, and M. Patterson (2021). Atlantic Meridional Overturning Circulation: Reviews of Observational and Modeling Advances-An Introduction. Journal of Geophysical Research: Oceans 126(1), e2020JC016745.

Steinfeldt, R., M. Rhein, J. L. Bullister, and T. Tanhua (2009). Inventory changes in anthropogenic carbon from 1997-2003 in the atlantic ocean between $20 \mathrm{~s}$ and 65 n. Global Biogeochemical Cycles 23(3).

Stevenson, S., B. Otto-Bliesner, J. Fasullo, and E. Brady (2016). "El Niño like" hydroclimate responses to last millennium volcanic eruptions. Journal of Climate 29(8), 2907-2921.

Stevenson, S., J. T. Overpeck, J. Fasullo, S. Coats, L. Parsons, B. Otto-Bliesner, T. Ault, G. Loope, and J. Cole (2018). Climate variability, volcanic forcing, and last millennium hydroclimate extremes. Journal of Climate 31(11), 4309-4327.

Stocker, T. F. and D. G. Wright (1991). Rapid transitions of the ocean's deep circulation induced by changes in surface water fluxes. Nature 351(6329), 729-732. 
Stommel, H. (1948). The westward intensification of wind-driven ocean currents. Eos, Transactions American Geophysical Union 29(2), 202-206.

Stommel, H. (1957). A survey of ocean current theory. Deep Sea Research (1953) 4, 149-184.

Stommel, H. (1958). The abyssal circulation. Deep-Sea Res. 5, 80-82.

Stommel, H. (1961). Thermohaline convection with two stable regimes of flow. Tellus 13(2), 224-230.

Stommel, H. (1965). The gulf stream: A physical and dynamical description, 248 pp. Univ. of Calif. Press, Berkeley.

Stramma, L. (1991). Geostrophic transport of the South Equatorial Current in the Atlantic. Journal of Marine Research 49(2), 281-294.

Stramma, L. and M. England (1999). On the water masses and mean circulation of the South Atlantic Ocean. Journal of Geophysical Research 104, 20,86320,883 .

Stramma, L., Y. Ikeda, and R. G. Peterson (1990). Geostrophic Transport in the Brazil Current region north of 20 degrees S. Deep-Sea Research Part A Oceanographic Research Papers 37(12), 1875-1886.

Stramma, L. and R. G. Peterson (1990). The South Atlantic Current. Journal of Physical Oceanography 20, 846-859.

Stramma, L. and F. Schott (1996). Western equatorial circulation and interhemispheric exchange. Gebr "u der Borntraeger.

Stramma, L. and F. Schott (1999). The mean flow field of the tropical atlantic ocean. Deep Sea Research Part II: Topical Studies in Oceanography 46(1-2), 279-303.

Sutton, R. T. and D. L. Hodson (2005). Atlantic ocean forcing of north american and european summer climate. science 309(5731), 115-118. 
Sverdrup, H. U. (1947). Wind-driven currents in a baroclinic ocean; with application to the equatorial currents of the eastern pacific. Proceedings of the National Academy of Sciences of the United States of America 33(11), 318.

Talley, L. D. (2003). Shallow, Intermediate, and Deep Overturning Components of the Global Heat Budget. Journal of Physical Oceanography 33, 530-560.

Talley, L. D. (2011). Descriptive physical oceanography: an introduction. Academic press.

Talley, L. D. (2013). Closure of the global overturning circulation through the indian, pacific, and southern oceans: Schematics and transports. Oceanography 26(1), 80-97.

Tamsitt, V., R. Abernathey, M. Mazloff, J. Wang, and L. Talley (2018). Transformation of deep water masses along lagrangian upwelling pathways in the southern ocean. Journal of Geophysical Research: Oceans 123(3), 1994-2017.

Tamsitt, V., H. F. Drake, A. K. Morrison, L. D. Talley, C. O. Dufour, A. R. Gray, S. M. Griffies, M. R. Mazloff, J. L. Sarmiento, J. Wang, et al. (2017). Spiraling pathways of global deep waters to the surface of the southern ocean. Nature communications 8(1), 1-10.

Thompson, D. W., S. Solomon, P. J. Kushner, M. H. England, K. M. Grise, and D. J. Karoly (2011). Signatures of the antarctic ozone hole in southern hemisphere surface climate change. Nature geoscience 4(11), 741-749.

Thompson, D. W. J. and S. Solomon (2002). Interpretation of recent Southern Hemisphere climate change. Science (New York, N.Y.) 296(5569), 895-899.

Thornalley, D. J., D. W. Oppo, P. Ortega, J. I. Robson, C. M. Brierley, R. Davis, I. R. Hall, P. Moffa-Sanchez, N. L. Rose, P. T. Spooner, et al. (2018). Anomalously weak labrador sea convection and atlantic overturning during the past 150 years. Nature 556(7700), 227-230.

Ting, M., Y. Kushnir, R. Seager, and C. Li (2011). Robust features of atlantic multidecadal variability and its climate impacts. Geophysical Research Letters 38(17). 
Toggweiler, J. and B. Samuels (1995). Effect of drake passage on the global thermohaline circulation. Deep Sea Research Part l: Oceanographic Research Papers 42(4), 477-500.

Tokinaga, H. and S.-p. Xie (2011). Weakening of the equatorial Atlantic cold tongue over the past six decades. Nature Geoscience 4, 222-226.

Trenberth, K. E. and J. M. Caron (2001). Estimates of meridional atmosphere and ocean heat transports. Journal of Climate 14(16), 3433-3443.

Trenberth, K. E., Y. Zhang, J. T. Fasullo, and L. Cheng (2019). Observationbased estimates of global and basin ocean meridional heat transport time series. Journal of Climate 32(14), 4567-4583.

Trouet, V., J. Esper, N. E. Graham, A. Baker, J. D. Scourse, and D. C. Frank (2009). Persistent positive north atlantic oscillation mode dominated the medieval climate anomaly. science 324(5923), 78-80.

Trouet, V., J. Scourse, and C. Raible (2012). North atlantic storminess and atlantic meridional overturning circulation during the last millennium: Reconciling contradictory proxy records of nao variability. Global and Planetary Change 84, 48-55.

Van Aken, H. M. (2007). The oceanic thermohaline circulation: an introduction, Volume 39. Springer Science \& Business Media.

Vazquez, H. (2018). Evaluating changes to natural variability on a warming globe in cmip5 models.

Vellinga, M., R. A. Wood, and J. M. Gregory (2002). Processes governing the recovery of a perturbed thermohaline circulation in hadcm3. Journal of Climate 15(7), 764-780.

Vellinga, M. and P. Wu (2004). Low-latitude fresh water influence on centennial variability of the thermohaline circulation. J. Climate 17(23), 4498-4511.

Visbeck, M. (2007). Power of pull. Nature 447(7143), 383-383. 
Wainer, I. and P. R. Gent (2019). Changes in the Atlantic Sector of the Southern Ocean estimated from the CESM Last Millennium Ensemble. Antarctic Science 31(1), 37-51.

Wainer, I., A. Taschetto, B. L. Otto-bliesner, and E. Brady (2004). A Numerical study of the impact of greenhouse gases on the South Atlantic Ocean climatology. Climatic Change 66, 163-189.

Wainer, I. and S. A. Venegas (2002). South Atlantic Multidecadal Variability in the Climate System Model. Journal of Climate 15(12), 1408-1420.

Wanamaker, A. D., P. G. Butler, J. D. Scourse, J. Heinemeier, J. Eiríksson, K. L. Knudsen, and C. A. Richardson (2012). Surface changes in the North Atlantic meridional overturning circulation during the last millennium. Nature Communications 3(1), 1-7.

Wang, G., W. Cai, and A. Purich (2014). Trends in Southern Hemisphere winddriven circulation in CMIP5 models over the 21st century: Ozone recovery versus greenhouse forcing. Journal of Geophysical Research: Oceans 119(5), 2974-2986.

Wang, J., B. Yang, F. C. Ljungqvist, J. Luterbacher, T. J. Osborn, K. R. Briffa, and E. Zorita (2017). Internal and external forcing of multidecadal atlantic climate variability over the past 1,200 years. Nature Geoscience 10(7), 512.

Warren, B. (1981). Deep circulation of the world ocean, evolution of physical oceanography ba warren, c. wunsch, 6-41.

Warren, B. A. (1983). Why is no deep water formed in the north pacific? Journal of Marine Research 41(2), 327-347.

Watson, A. J., U. Schuster, J. D. Shutler, T. Holding, I. G. Ashton, P. Landschützer, D. K. Woolf, and L. Goddijn-Murphy (2020). Revised estimates of oceanatmosphere co 2 flux are consistent with ocean carbon inventory. Nature communications 11(1), 1-6. 
Weijer, W., W. Cheng, S. S. Drijfhout, A. V. Fedorov, A. Hu, L. C. Jackson, W. Liu, E. McDonagh, J. Mecking, and J. Zhang (2019). Stability of the Atlantic meridional overturning circulation: A review and synthesis. Journal of Geophysical Research: Oceans 124(8), 5336-5375.

Weijer, W., W. Cheng, O. A. Garuba, A. Hu, and B. Nadiga (2020). CMIP6 models predict significant 21st century decline of the Atlantic Meridional Overturning Circulation. Geophysical Research Letters 47(12), e2019GL086075.

Weiss, R. (1974). Carbon dioxide in water and seawater: the solubility of a non-ideal gas. Marine chemistry 2(3), 203-215.

Wells, N. C. (2012). The atmosphere and ocean: a physical introduction.

Whitworth III, T. (1983). Monitoring the transport of the antarctic circumpolar current at drake passage. Journal of Physical Oceanography 13(11), 20452057.

Wienders, N., M. Arhan, and H. Mercier (2000). Circulation at the western boundary of the south and equatorial atlantic: Exchanges with the ocean interior. Journal of Marine Research 58(6), 1007-1039.

Williams, R. G. and M. J. Follows (2011). Ocean dynamics and the carbon cycle: Principles and mechanisms. Cambridge University Press.

Winton, M., S. M. Griffies, B. L. Samuels, J. L. Sarmiento, and T. L. Frölicher (2013). Connecting changing ocean circulation with changing climate. Journal of climate $26(7), 2268-2278$.

Witter, D. L. and A. L. Gordon (1999). Interannual variability of South Atlantic circulation from 4 years of TOPEX/POSEIDON satellite altimeter observations. Journal of Geophysical Research 104(C9), 20,927-20,948.

Wood, R. A., J. M. Rodríguez, R. S. Smith, L. C. Jackson, and E. Hawkins (2019). Observable, low-order dynamical controls on thresholds of the atlantic meridional overturning circulation. Climate Dynamics 53(11), 6815-6834. 
Worthington, E. L., B. I. Moat, D. A. Smeed, J. V. Mecking, R. Marsh, and G. D. McCarthy (2021). A 30-year reconstruction of the atlantic meridional overturning circulation shows no decline. Ocean Science 17(1), 285-299.

Wu, L., W. Cai, L. Zhang, H. Nakamura, A. Timmermann, T. Joyce, M. J. McPhaden, M. Alexander, B. Qiu, M. Visbeck, P. Chang, and B. Giese (2012, jan). Enhanced warming over the global subtropical western boundary currents. Nature Climate Change 2(3), 161-166.

Wunsch, C. (2002). What is the thermohaline circulation? Science 298(5596), $1179-1181$.

Wunsch, C. and R. Ferrari (2004). Vertical mixing, energy, and the general circulation of the oceans. Annu. Rev. Fluid Mech. 36, 281-314.

Wunsch, C. and R. Ferrari (2018). 100 years of the ocean general circulation. Meteorological Monographs 59, 7-1.

Xu, X., E. P. Chassignet, and F. Wang (2019). On the variability of the atlantic meridional overturning circulation transports in coupled cmip5 simulations. Climate Dynamics 52(11), 6511-6531.

Yamagami, Y. and T. Tozuka (2015, dec). Interannual variability of South Equatorial Current bifurcation and western boundary currents along the Madagascar coast. Journal of Geophysical Research: Oceans 120(12), 8551-8570.

Yamamoto, A., J. B. Palter, M. S. Lozier, M. S. Bourqui, and S. J. Leadbetter (2015). Ocean versus atmosphere control on western european wintertime temperature variability. Climate dynamics 45(11), 3593-3607.

Yang, H., G. Lohmann, U. Krebs-Kanzow, M. Ionita, X. Shi, D. Sidorenko, X. Gong, X. Chen, and E. J. Gowan (2020). Poleward shift of the major ocean gyres detected in a warming climate. Geophysical Research Letters 47(5), e2019GL085868.

Yang, H., G. Lohmann, W. Wei, M. Dima, M. Ionita, and J. Liu (2016). Intensification and poleward shift of subtropical western boundary currents in a warming climate. Journal of Geophysical Research: Oceans 121(7), 4928-4945. 
Yeager, S. (2015). Topographic coupling of the atlantic overturning and gyre circulations. Journal of Physical Oceanography 45(5), 1258-1284.

Yeager, S., G. Danabasoglu, N. Rosenbloom, W. Strand, S. Bates, G. Meehl, A. Karspeck, K. Lindsay, M. Long, H. Teng, et al. (2018). Predicting near-term changes in the earth system: A large ensemble of initialized decadal prediction simulations using the community earth system model. Bulletin of the American Meteorological Society 99(9), 1867-1886.

Yeager, S. and J. Robson (2017). Recent progress in understanding and predicting atlantic decadal climate variability. Current Climate Change Reports 3(2), 112127.

You, Y. (2002). Quantitative estimate of antarctic intermediate water contributions from the drake passage and the southwest indian ocean to the south atlantic. Journal of Geophysical Research: Oceans 107(C4), 6-1.

Zambri, B., A. N. LeGrande, A. Robock, and J. Slawinska (2017). Northern hemisphere winter warming and summer monsoon reduction after volcanic eruptions over the last millennium. Journal of Geophysical Research: Atmospheres 122(15), 7971-7989.

Zhai, F., D. Hu, Q. Wang, and F. Wang (2014). Long-term trend of pacific south equatorial current bifurcation over 1950-2010. Geophysical Research Letters 41(9), 3172-3180.

Zhang, D., M. J. McPhaden, and W. E. Johns (2003). Observational Evidence for Flow between the Subtropical and Tropical Atlantic: The Atlantic Subtropical Cells. Journal of Physical Oceanography 33, 1783-1797.

Zhang, D., R. Msadek, M. J. McPhaden, and T. Delworth (2011, apr). Multidecadal variability of the North Brazil Current and its connection to the Atlantic meridional overturning circulation. Journal of Geophysical Research 116(C4), C04012.

Zhang, L. and T. Qu (2015). Low-frequency variability of the south pacific subtropical gyre as seen from satellite altimetry and argo. Journal of Physical Oceanography 45(12), 3083-3098. 
Zhang, R., R. Sutton, G. Danabasoglu, Y.-O. Kwon, R. Marsh, S. G. Yeager, D. E. Amrhein, and C. M. Little (2019). A review of the role of the Atlantic meridional overturning circulation in Atlantic multidecadal variability and associated climate impacts. Reviews of Geophysics 57(2), 316-375.

Zhang, X., J. a. Church, S. M. Platten, and D. Monselesan (2013, aug). Projection of subtropical gyre circulation and associated sea level changes in the Pacific based on CMIP3 climate models. Climate Dynamics 43(1-2), 131-144.

Zhu, C. and Z. Liu (2020). Weakening Atlantic overturning circulation causes South Atlantic salinity pile-up. Nature Climate Change 10(11), 998-1003.

Zhu, J., Z. Liu, J. Zhang, and W. Liu (2015). AMOC response to global warming: Dependence on the background climate and response timescale. Climate Dynamics 44(11-12), 3449-3468. 


\section{Appendix I}

- (1) Figure analogous to Figure 31a, b - referenced in Section 5.3.4.

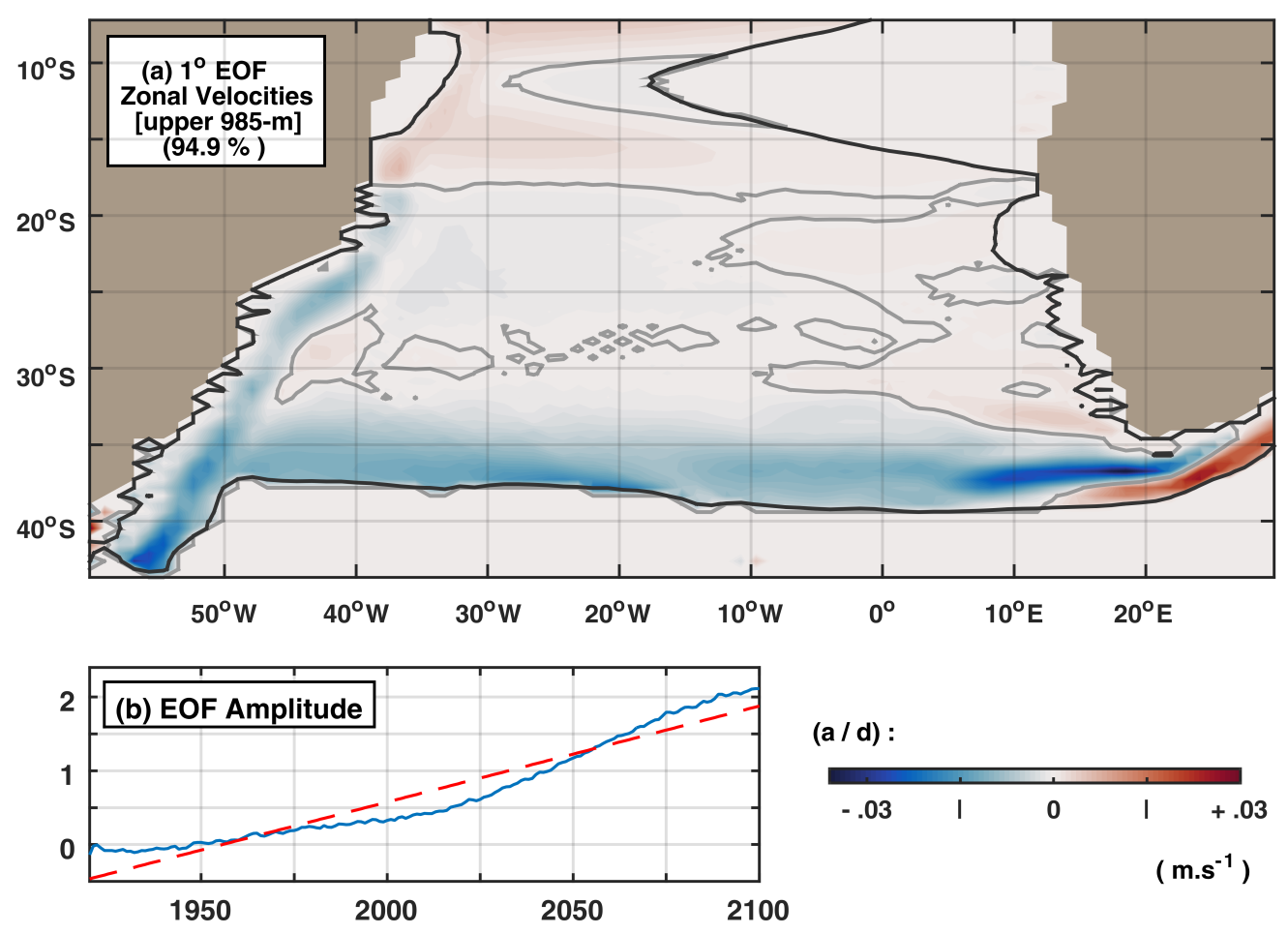

Figure 38 (a) Spatial pattern of the leading EOF (94.9\% of total variance) of non-detrended zonal velocity anomalies (1920-1970 base period) during 1920-2100 within the upper 985-m of the South Atlantic subtropical gyre (delimited by the 1920-2100 mean zero zonal velocity line - black filled contour). (b) Corresponding dimensionless amplitude (principal component, PC) time series.

Items attached as external files (Appendix I - 2-4.zip):

- (2) Supporting Information for "South Atlantic subtropical gyre late twentieth century changes" (Marcello et al., 2018)

- (3) Supplementary Materials for "South Atlantic Surface Boundary Current System during the Last Millennium in the CESM-LME: The Medieval Climate Anomaly and Little Ice Age" (Marcello et al., 2019)

- (4) Manuscript draft - "21st Century Projected Changes in the Weddell Sea Antarctic Slope Current in a Downscaling Experiment" 


\title{
Supporting Information for "South Atlantic Subtropical Gyre late twentieth century changes"
}

\author{
Fernanda Marcello ${ }^{1}$, Ilana Wainer ${ }^{1}$, Regina R. Rodrigues ${ }^{2}$, \\ ${ }^{1}$ Department of Physical Oceanography, Oceanographic Institute of the University of São Paulo, \\ São Paulo, SP, Brazil \\ ${ }^{2}$ Department of Geosciences, Federal University of Santa Catarina, Florianópolis, SC, Brazil
}

\section{Contents}

1. Text S1 - Validation and Characterization

2. Text S2 - Statistical analysis

3. Figure S1- sSEC bifurcation vertical profile

4. Figure $\mathrm{S} 2$ - SBL surface position

5. Figure S3- Depth-integrated flow over the upper $50 \mathrm{~m}$

6. Figure S4- Low frequency SASG variability

7. Figure S5- SBL anomalies at $100 \mathrm{~m}$

8. Figure $\mathrm{S6}$ - Schematic of the South Atlantic basin-scale controlling dynamics

9. Table $\mathrm{S1}$ - Ocean reanalysis products

10. Table $\mathrm{S2}$ - SBL values

11. Table $\mathrm{S} 3$ - Comparison of the SBL shift

\section{Introduction}

The auxiliary materials contain the model validation results, which consist of the detailed comparison of the sSEC bifurcation vertical profile and the depth-integrated flow over the upper $50 \mathrm{~m}$ between the CESM-OCN model and five ocean reanalysis products.

A comparison between the southward migration of the SBL in the SAO with the change in position of the Pacific NBL and SBL from other studies is presented in Table S3.

We also provide a detailed version of Figure 3 from the main text. Therefore Figure $\mathrm{S} 4$ in this supporting information shows the time series of the mean WSC/SSH/BSF inside their zero contour and of their corresponding area as well, which were used to produce the final time series presented in Figure 3 from the main text, that represents the "mean WSC/SSH/BSF per area". Furthermore, Figure S4 also shows these time series for further confined contours inside the zero contours: i.e., of $5 \times 10^{-8} \mathrm{~N} \mathrm{~m}^{-3}$ for the WSC, $15 \mathrm{~cm}$ for the SSH and $-10 \mathrm{~Sv}$ for the BSF. The WSC and BSF (SSH) strength hold an indirect (direct) relation with their filling areas (with correlation coefficients reaching up to -0.58 for the WSC in Figure $\mathrm{S} 44,+0.90$ for the SSH in Figure $\mathrm{S} 4 \mathrm{~d}$ and -0.58 for the BSF in Figure $\mathrm{S} 4 \mathrm{4}$ ). However, the resulting time series "mean intensity per area" is not substantially affected by this and does not change the conclusions that the WSC, SSH and BSF strengths are indeed increasing.

The time series of the SBL anomalies at $100 \mathrm{~m}$ depth, which is mentioned in the main text, is displayed in Figure $\$ 5$.

Corresponding author: Fernanda Marcello, fernanda.marcello@usp.br 
Finally, Figure \$6 provides a comprehensive framework of the dynamical fields integration through two complementary panels. The top panel shows superimposed zero contours for the WSC, SSH and BSF, with the WSC as background colors; while the lower panel displays superimposed contours of SVT0, WSC0 and BSF0, with the SVT as background colors.

\section{Text S1 - Validation and Characterization.}

To investigate how well the model simulates the subtropical gyre dynamics and the sSEC bifurcation, the results were compared to those of the ocean 11 reanalysis products, which can be directly accessed through these links:

1. ORAS4 - http://icdc.cen.uni-hamburg.de/1/projekte/easy-init/easy-init-ocean.html?no_cache=1

2. ERA-Interim - http://apps.ecmwf.int/datasets/data/interim-full-moda/levtype=sfc/

3. GFDL - https://www.gfdl.noaa.gov/ocean-data-assimilation-model-output/

4. SODA216 - http://apdrc.soest.hawaii.edu/datadoc/soda_2.1.6.php

5. GODAS - http://iridl.ldeo.columbia.edu/SOURCES/.NOAA/.NCEP/.EMC/.CMB/.GODAS/.monthly/

6. CFSR - https://rda.ucar.edu/datasets/ds093.2/\#! access

The comparisons were made based on the periods covered by the reanalysis products, from 1970 on (Table S1) and on the period of 1970-2015 for the CESM-OCN.

The meridional velocity averaged within a $4^{\circ}$ longitude band off the South American coast was compared (Figure S1); the thick-black contours represent the SBL. All data sets seem to depict the depth dependence of the bifurcation latitude well, although slightly different configurations of the sSEC bifurcation vertical profile can be noted between them.

From hydrographic observations and numerical model results, R2007 characterized the sSEC bifurcation vertical structure at the western boundary, providing a description of its annual mean depth dependence. The authors showed that the bifurcation occurs at approximately $10^{\circ}-14^{\circ} \mathrm{S}$ in the top $100 \mathrm{~m}$ and shifts poleward with increasing depth, reaching $27^{\circ} \mathrm{S}$ at $1000 \mathrm{~m}$ (Figure 3 from R2007).

Among the reanalysis products that were examined, the structure that more closely resembles the contour from the R2007 hydrographic observations is the one reproduced by ORAS4. A previous investigation that evaluated the products' performance in terms of the mean circulation field for different levels, in comparison to the available observations of R2007 (not shown), also suggested that ORAS4 better captures the dynamics of the SBL.

ORAS4 also captures the Pacific SBL interannual variation reasonably well, as shown by Yamagami and Tozuka (2015). The authors compared the SBL derived from ORAS4 with the one calculated from the surface meridional velocity obtained from AVISO derived data, although it has been remarked by the authors that, since ORAS4 assimilates observed sea level anomalies, it is not completely independent of the AVISO derived data.

The mean SBL for individual levels up to $600 \mathrm{~m}$ is listed in Table $\$ 2$, Despite some small differences, all data-sets reproduced the poleward tilting of the sSEC bifurcation with increasing depth. The total shift up to $600 \mathrm{~m}$ of the R2007 observations and of the ORAS4 and CESM-OCN results are within the range of $9.6^{\circ}-13.1^{\circ}$.

\footnotetext{
${ }^{1} 1$ - and winds from the ERA-Interim atmospheric product with the ORAS4 ocean product.
} 
For the SBL at the surface (sSBL), which is the focus of this study, the CESMOCN value falls close to the values from the R2007 observations and from ORAS4 and SODA216 reanalysis (Figure $\$ 2$ ).

Considering the large scale climatology, a good correspondence between the SBL and the zero zonally integrated WSC line is expected. That is because, according to the Sverdrup balance (Pedlosky, 1996), the sSEC should bifurcate along this line - at the latitude where no western boundary current compensates the total interior transport. Figure $\mathrm{S} 3$ demonstrates this relationship between the bifurcation latitude in the upper ocean and the large scale basin-integrated WSC, showing the mean horizontal flow field, vertically integrated over the top $50 \mathrm{~m}$ as vectors. The ERA-Interim atmospheric product (used in conjunction with the current velocities from the ORAS4 ocean product) and the CESM-OCN reasonably attain the Sverdrup balance in the upper-ocean layer, as can be seen in Figure $\{3 \mathrm{p}$, f.

Therefore, the simulation results from CESM-OCN are similar to the results derived from ORAS4 and the R2007 hydrographic observations, proving that the model simulates the SSEC bifurcation and the region of study well.

These results also agree with the available literature. From hydrographic data, Stramma and England [1999] showed that the bifurcation latitude is $16^{\circ} \mathrm{S}$ in the nearsurface layer (top $100 \mathrm{~m}$ ), $20^{\circ} \mathrm{S}$ in the South Atlantic Central Water (SACW) layer $(100-500 \mathrm{~m})$, and $26^{\circ} \mathrm{S}$ in the intermediate layer $(500-1200 \mathrm{~m})$. Using isobaric RAFOS floats, Boebel et al. [1999] showed that the Return Current (analog to the SEC, but within the Antarctic Intermediate Water (AAIW) layer) reaches the South American coast at approximately $28^{\circ} \mathrm{S}$ (called the Santos Bifurcation by the authors). Using data from the World Ocean Circulation Experiment (WOCE) hydrographic section A17, which was taken during the austral summer of 1994, Wienders et al. [2000] estimated the transport of the SEC and its bifurcation latitude for several isopycnal layers: the SEC bifurcation latitude is $14^{\circ} \mathrm{S}$ at the surface, $24^{\circ} \mathrm{S}$ in the 26.7-26.9 layer $(400-500 \mathrm{~m})$, and nearly constant around $26^{\circ}-28^{\circ} \mathrm{S}$ in the AAIW and Upper Circumpolar Water (UCPW: 600-1200 m). These results should be interpreted with caution because they are based on a single hydrographic section taken from $6^{\circ}-10^{\circ}$ from the western boundary. In the simulations by Harper [2000], the bifurcation point in the near-surface layer at the western boundary of the SAO occurs at $18^{\circ} \mathrm{S}$, and in those by Malanotte-Rizzoli et al. [2000], it occurs at $17^{\circ} \mathrm{S}$. From the results of two high-resolution ocean global circulation models (OGCMs), the Hybrid Coordinate Ocean Model - HYCOM and the Ocean Circulation and Climate Advanced Modeling Project - OCCAM, Pereira et al. [2014] found that the latitude of bifurcation of the zonal flows reaching the coast (analog to the SEC), is $13^{\circ}-15^{\circ} \mathrm{S}$ for the Tropical Water, $22^{\circ} \mathrm{S}$ for the Central Water, $28^{\circ}-30^{\circ} \mathrm{S}$ for the Antarctic Intermediate Water. Cirano et al. [2006], using data from the global circulation model Ocean Circulation and Climate Advanced Modeling Project (OCCAM), found that the bifurcation occurs between $9^{\circ}$ $15^{\circ} \mathrm{S}$ in the TW $(0-116 \mathrm{~m})$, migrating to $25^{\circ} \mathrm{S}$ in the SACW (116-657 m) and $25^{\circ}-30^{\circ} \mathrm{S}$ in the AAIW (657-1234 m).

For the boundary currents mean volume transports (13.11 Sv, $12.53 \mathrm{~Sv}$ and 4.32 Sv for the sSEC, NBUC and BC in this study, respectively), R2007 found an average transport of $15 \mathrm{~Sv}$ for the SSEC and $14 \mathrm{~Sv}$ for the NBUC; integrated up to $400 \mathrm{~m}$ across the same coordinates and between the same transects, while for the $\mathrm{BC}$, across $22^{\circ} \mathrm{S}$, the authors found an average transport of $6 \mathrm{~Sv}$. Schott et al. [1998] found an average transport of $14.6 \mathrm{~Sv}$ for the NBUC across $5^{\circ} \mathrm{S}$ (above the 26.8 isopycnal) and Stramma and Peterson [1990] found a $16 \mathrm{~Sv}$ value for the sSEC across $30^{\circ} \mathrm{W}$ (upper $400 \mathrm{~m}$ ). For the BC, Evans and Signorini [1985] found a $5.5 \mathrm{~Sv}$ value across $25^{\circ} \mathrm{S}$ (upper $400 \mathrm{~m}$ ), while Stramma et al. [1990] found a $4 \mathrm{~Sv}$ value across $20^{\circ} \mathrm{S}$ (upper 500 $\mathrm{m})$. 


\section{Text S2 - Statistical analysis}

To reduce random noise while retaining a sharp step response of the time series (extracting, therefore, the low-frequency component), a multiple-pass moving average (or multi-running mean) filter was applied, which involves passing the input signal through a moving average filter two or more times (e.g., Smith [1997]). This procedure compared to the use of regular annual means, for instance, did not change our results. The low-passed time series in the manuscript are 15-month multi-running means, unless otherwise specified.

The long-term trends were estimated by least squares fit and their statistical significance were estimated with the Mann-Kendall test [Kendall, 1975; Mann, 1945], at the $95 \%$ or $99 \%$ confidence level. This is a non-parametric method less affected by the outliers sometimes contained in data (e.g., Tokinaga and Xie [2011]). If the Student's $t$-test is applied to the same data, our conclusions do not change.

To quantify the linear relations between time series, the Pearson's correlation coefficient was used (see Rodgers and Nicewander [1988], for detailed information). To determine if the correlations were significant a t-test was applied. All the correlations mentioned in this study are statistically significant at the $95 \%$ or $99 \%$ confidence level. 
Figure S1

(a) ORAS4

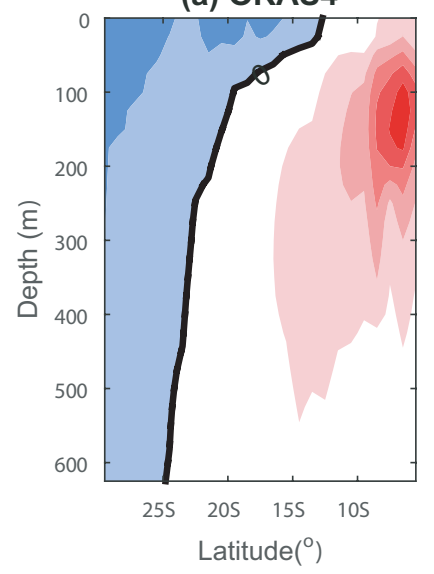

(d) GODAS

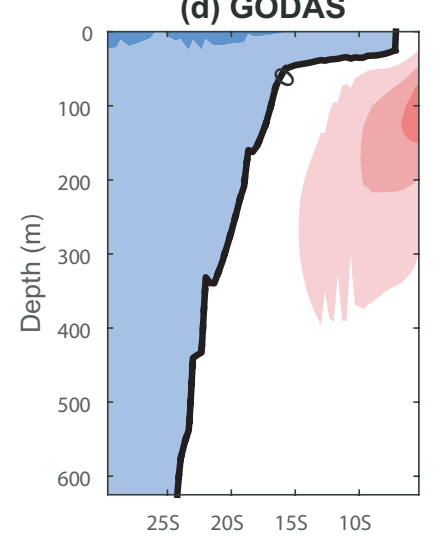

(b) GFDL

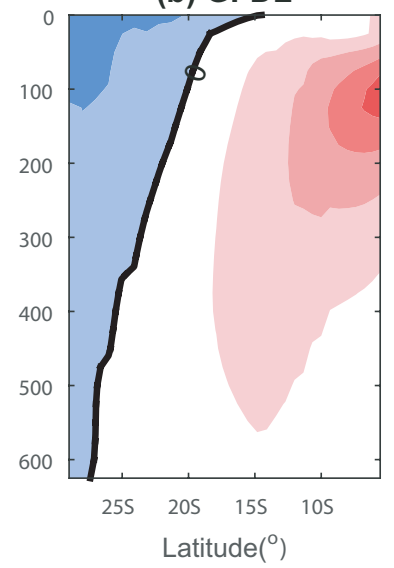

(e) CFSR

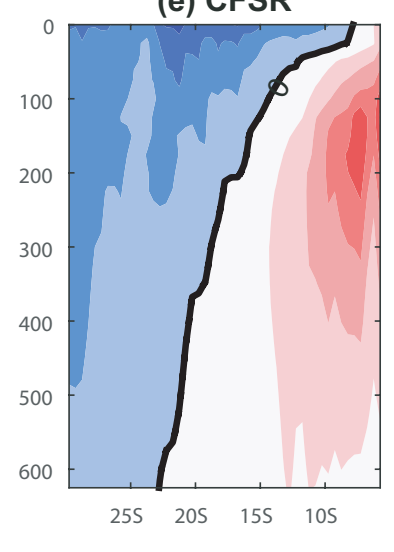

(c) SODA216

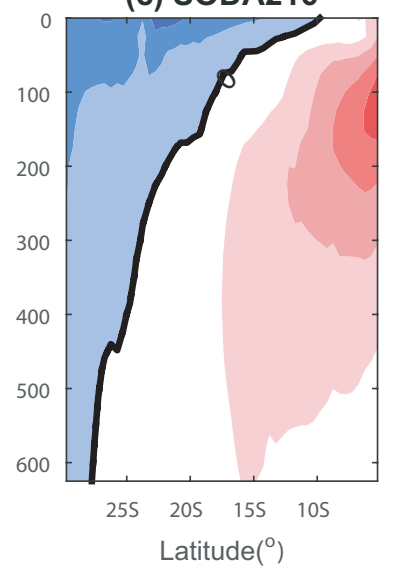

(f) CESM-OCN

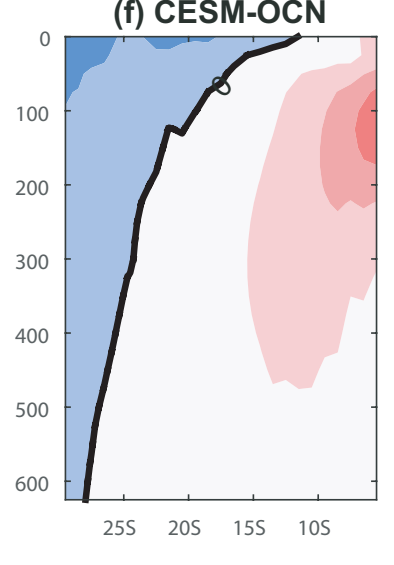

Meridional Velocity (m.s $\mathrm{s}^{-1}$ )

| sSEC bifurcation vertical profile from the reanalysis products and model results. Mean meridional velocity $\left(\mathrm{m} \mathrm{s}^{-1}\right)$ averaged over the western boundary layer $\left(<4^{\circ}\right.$ from the coast) from ocean reanalysis products (a-e) and model results (f-g). Positive (negative) values indicate northward (southward) flow associated with the NBUC (BC), and the contour of zero velocity represents the bifurcation of the sSEC. 


\section{Figure S2}

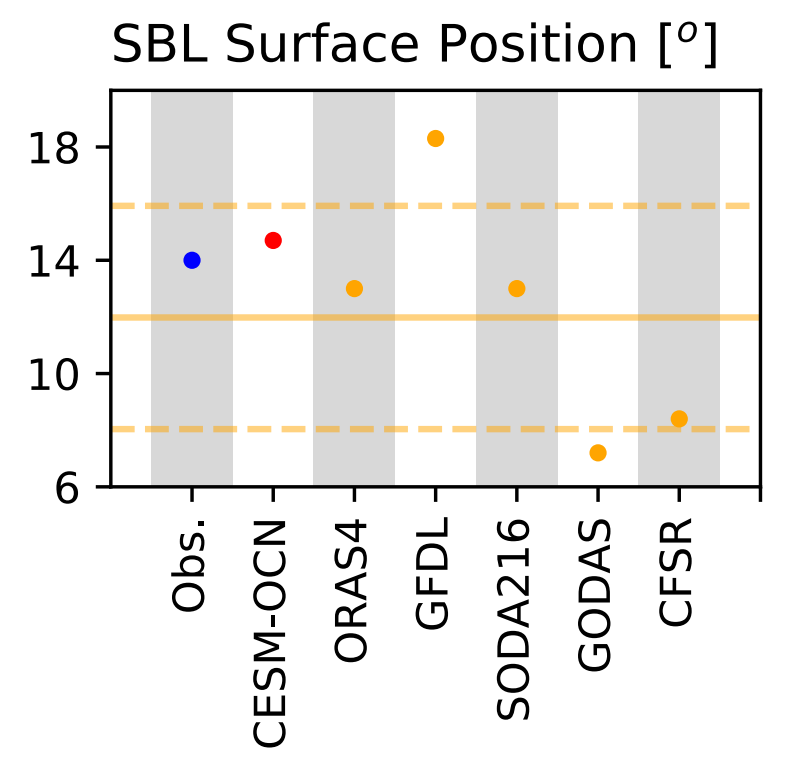

| SBL Surface Position $\left({ }^{\circ} \mathbf{S}\right)$. | From the available observations (blue dot), model results (red dot) and reanalysis products (yellow dots). The horizontal solid yellow line indicates the mean value for all the 5 reanalysis products and dashed yellow lines indicate the standard deviation range. 


\section{Figure S3}

(a) ORAS4

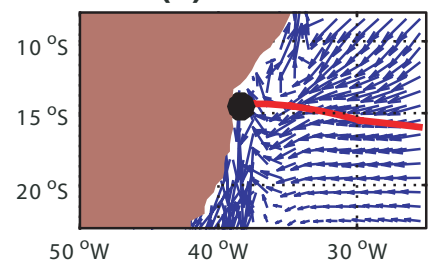

(d) GODAS

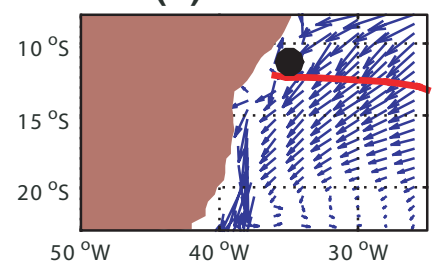

(b) GFDL

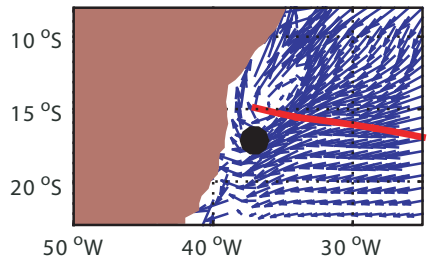

(e) CFSR

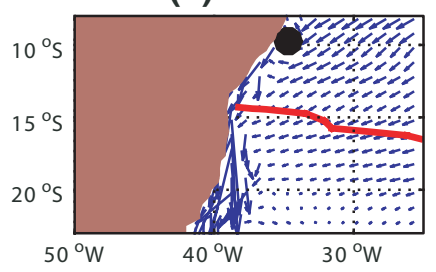

(c) SODA216

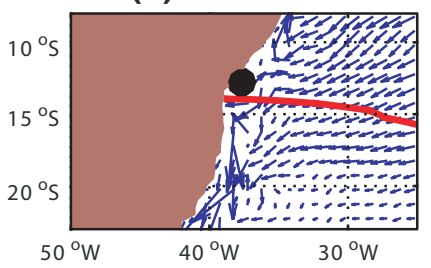

(f) CESM-OCN

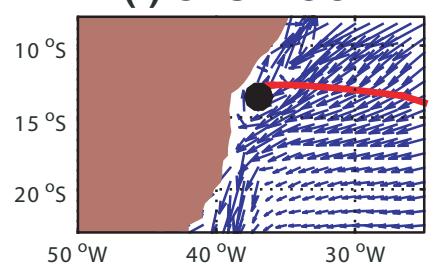

| Depth-integrated flow over the upper $50 \mathrm{~m}$ (blue vectors). | Derived from ocean reanalysis products - (a) ORAS4 flow field and ERA-Interim wind stress, (b) GFDL, (c) SODA216, (d) GODAS, and (e) CFSR - and from the model results - (f) CESM-OCN. The dark dots indicate the mean position of the SBL, and the red lines indicate the zero contour of the wind stress curl integrated from east to west. 


\section{Figure S4}
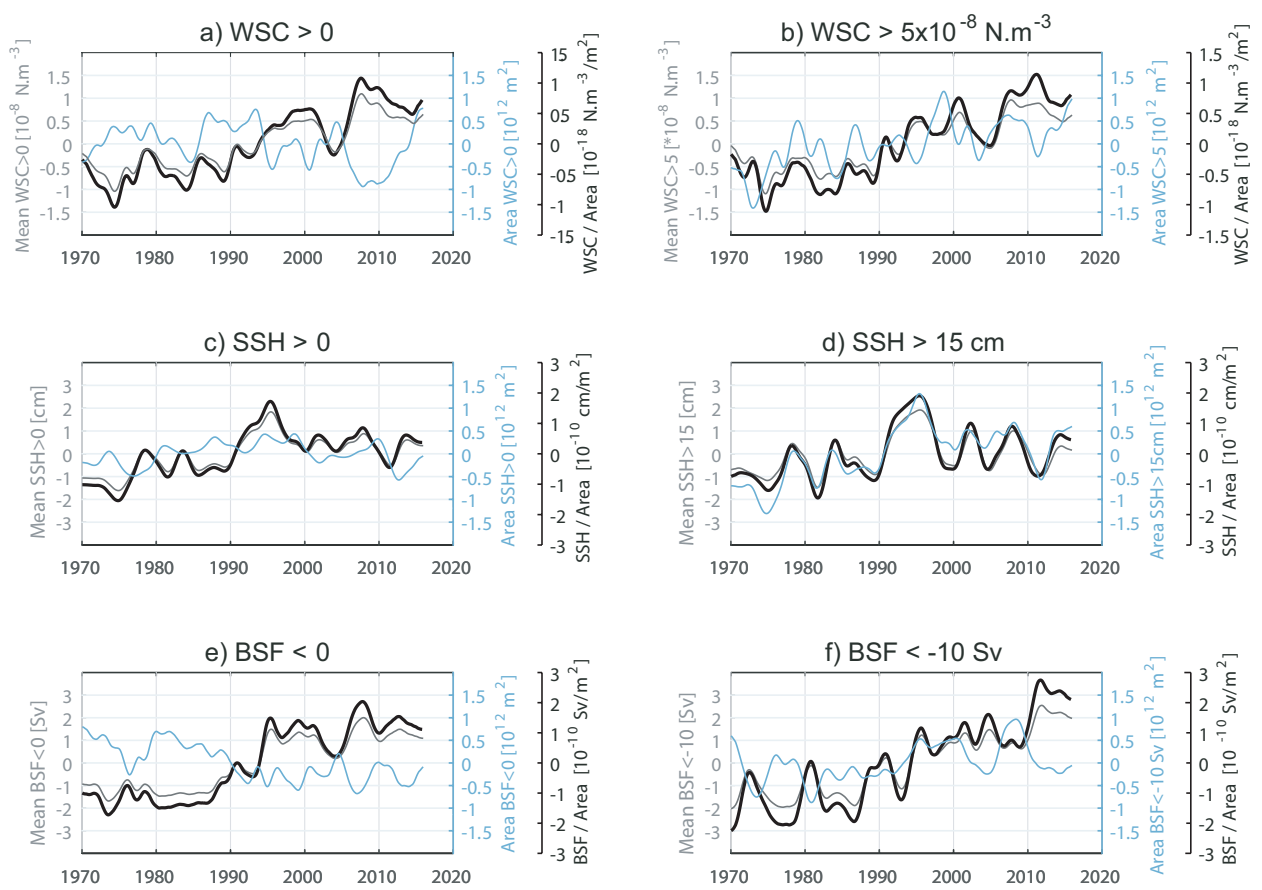

| Low-frequency SASG variability. | Low-passed anomalies of mean (a) WSC, (c) SSH and (e) BSF horizontally averaged inside their zero contour within the SASG (gray), of the corresponding area enclosed by their respective zero contour (light blue) and resulting time series of mean WSC/SSH/BSF per area (i.e., the gray divided by the blue line - in thick black lines). (b) (d) (f) Same as (a)/(c)/(e) but averaged inside the (b) $5 \times 10^{-8} \mathrm{~N} \mathrm{~m}^{-3}$ WSC line, (d) $15 \mathrm{~cm}$ SSH line, and (f) -10 Sv BSF line. 


\section{Figure S5}

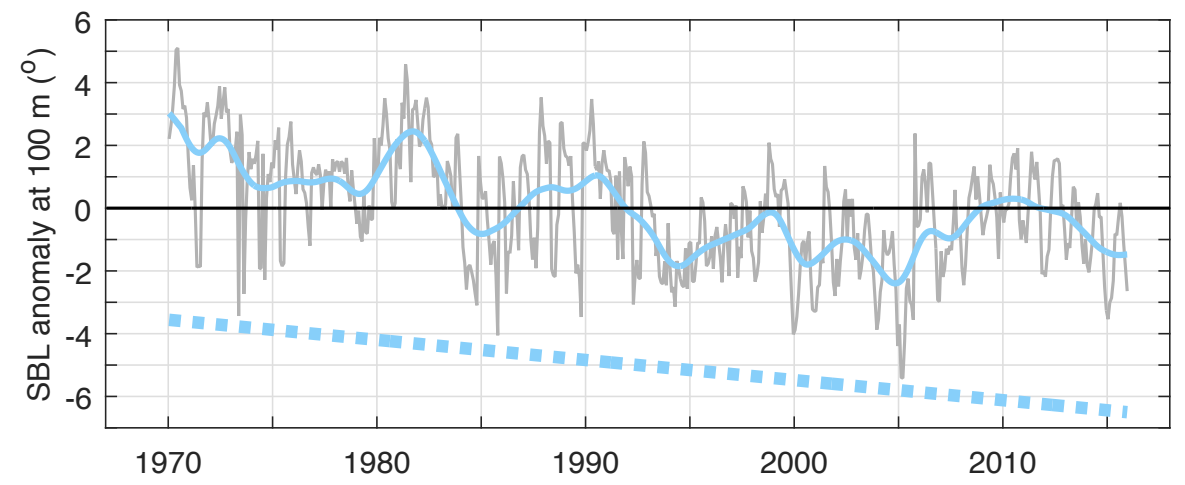

| Time series of the SBL anomalies at $100 \mathrm{~m}$. | Monthly means (light gray) overlaid by low-passed, 15-month multiple running means (light blue). The light blue dots indicate the linear trend, which is reduced by $5^{\circ}$ for clarity. The position used to derive the time series (100 $\mathrm{m}$ depth) is indicated by the light blue asterisk in Figure $7 \mathrm{~b}$ from the main text. 


\section{Figure S6}
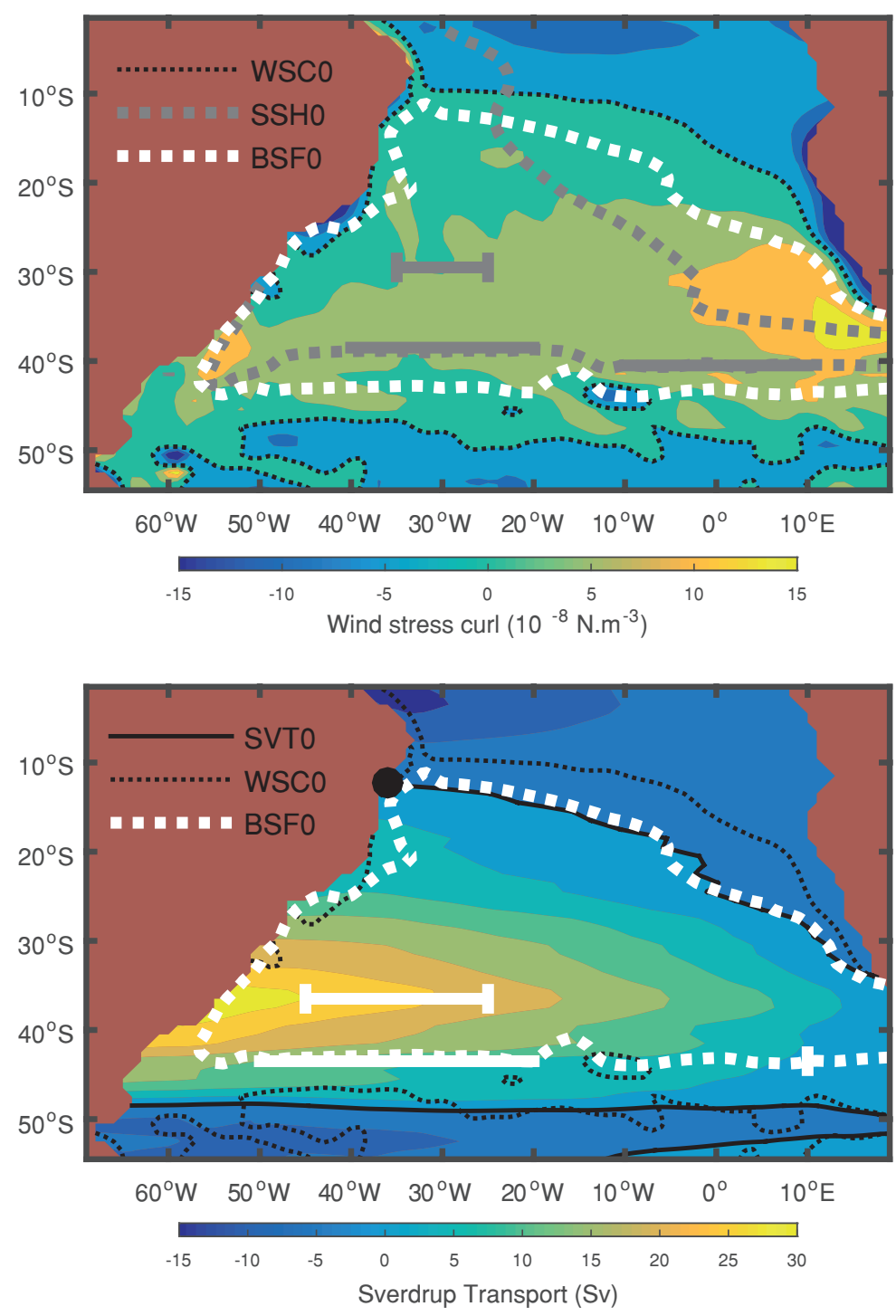

| Schematic of the South Atlantic basin-scale controlling dynamics. | (Top) Background colors show the mean WSC field (contour interval: $0.5 \mathrm{~N} \cdot \mathrm{m}^{-3}$ ), where positive values within the gyre are bounded by thin black dotted lines (WSC0 contour). Thick gray dashed lines demarcate the contour of SSH0, while gray zonal labels indicate the widths used to derive the time series of the position of maximum zonally averaged SSH $\left(35^{\circ}-25^{\circ} \mathrm{W}\right.$, center) and of the SSH0 at a western $\left(40^{\circ}-20^{\circ} \mathrm{W}\right)$ and an eastern portion of the basin $\left(10^{\circ} \mathrm{W}-10^{\circ} \mathrm{E}\right)$. Thick white dashed lines demarcate the contour of BSF0. (Bottom) Same as top panel but with mean SVT field as background colors (contour interval: $5 \mathrm{~Sv}$ ), where positive values within the gyre are bounded by the thick solid black line. The black dot centered at $36^{\circ} \mathrm{W}$ shows the location used to derive the SVT0 time series at the northern boundary. White labels denote the widths and location used to derive the time series of the position of maximum zonally averaged $|\mathrm{BSF}|\left(45^{\circ}-25^{\circ} \mathrm{W}\right.$, center) and of the BSF0 at a western $\left(50^{\circ}-20^{\circ} \mathrm{W}\right)$ and an eastern portion of the basin $\left(10^{\circ} \mathrm{E}\right)$. 


\section{Table S1}

Time period, horizontal resolution and number of vertical layers above $1000 \mathrm{~m}$ of the 5 ocean reanalysis products.

\begin{tabular}{cccc}
\hline Product & Period & Horiz. Resolution & Vert. Layers \\
\hline ORAS4 & $1970-2010$ & $1^{\circ} \times 1^{\circ}$ & 26 \\
GFDL & $1970-2010$ & $1^{\circ} \times 0.33^{\circ}-1^{\circ}$ & 34 \\
SODA216 & $1970-2008$ & $0.5^{\circ} \times 0.5^{\circ}$ & 22 \\
GODAS & $1980-2010$ & $1^{\circ} \times 1 / 3^{\circ}$ & 31 \\
CFSR & $1980-2010$ & $0.5^{\circ} \times 0.5^{\circ}$ & 31 \\
\hline
\end{tabular}

* ERA-Interim winds (1979:2010 period) were used with ORAS4 current velocities. 


\section{Table S2}

SBL values obtained by R2007 from hydrographic observations, derived from ocean reanalysis products and obtained from the CESM-OCN results.

\begin{tabular}{ccccccc}
\hline & Surface & $\mathbf{1 0 0} \mathbf{~}$ & $\mathbf{2 0 0} \mathbf{~ m}$ & $\mathbf{4 0 0} \mathbf{~ m}$ & $\mathbf{6 0 0} \mathbf{~ m}$ & Total shift \\
\hline Obs.* $^{*}$ & $14^{\circ} \mathrm{S}$ & $14^{\circ} \mathrm{S}$ & $18.6^{\circ} \mathrm{S}$ & $21^{\circ} \mathrm{S}$ & $23.6^{\circ} \mathrm{S}$ & $9.6^{\circ}$ \\
ORAS4 & $13^{\circ} \mathrm{S}$ & $19.6^{\circ} \mathrm{S}$ & $21.3^{\circ} \mathrm{S}$ & $23.3^{\circ} \mathrm{S}$ & $24.7^{\circ} \mathrm{S}$ & $11.7^{\circ}$ \\
GFDL & $18.3^{\circ} \mathrm{S}$ & $20.1^{\circ} \mathrm{S}$ & $21.2^{\circ} \mathrm{S}$ & $25.5^{\circ} \mathrm{S}$ & $27.1^{\circ} \mathrm{S}$ & $8.8^{\circ}$ \\
SODA216 & $13^{\circ} \mathrm{S}$ & $18.1^{\circ} \mathrm{S}$ & $22.1^{\circ} \mathrm{S}$ & $25.1^{\circ} \mathrm{S}$ & $27.6^{\circ} \mathrm{S}$ & $14.6^{\circ}$ \\
GODAS & $7.2^{\circ} \mathrm{S}$ & $16.9^{\circ} \mathrm{S}$ & $19^{\circ} \mathrm{S}$ & $22.2^{\circ} \mathrm{S}$ & $24.1^{\circ} \mathrm{S}$ & $16.9^{\circ}$ \\
CFSR & $8.4^{\circ} \mathrm{S}$ & $14.4^{\circ} \mathrm{S}$ & $16.5^{\circ} \mathrm{S}$ & $20.5^{\circ} \mathrm{S}$ & $22.6^{\circ} \mathrm{S}$ & $14.2^{\circ}$ \\
CESM-OCN & $14.7^{\circ} \mathrm{S}$ & $19^{\circ} \mathrm{S}$ & $23.3^{\circ} \mathrm{S}$ & $25.7^{\circ} \mathrm{S}$ & $27.8^{\circ} \mathrm{S}$ & $13.1^{\circ}$ \\
\hline
\end{tabular}

* Hydrographic observations from R2007: geostrophic velocities determined from dynamic heights relative to 1000 dbar, calculated with an annual mean climatology of temperature and salinity constructed from observations [quality-controlled CTD and bottle data obtained from HydroBase (Curry 1996)]. The authors' calculations do not include the Ekman current, which would affect the bifurcation latitude near the surface. They clarify that adding the Ekman currents to the geostrophic currents (calculated from observations) moves the bifurcation latitude northward by about $1^{\circ}$ (i.e., the bifurcation occurs at $13^{\circ} \mathrm{S}$ at the surface). 


\section{Table S3}

Comparison of the southward shift of the SAO SBL in this study with the Pacific NBL and SBL, from Chen and Wu [2012] and Zhai et al. [2014], respectively.

\begin{tabular}{ccccc}
\hline Bifurcation & Level/Layer & Period & Shift & Rate \\
\hline SAO SBL & surface & $1970-2015$ & from $11^{\circ}$ to $16^{\circ} \mathrm{S}$ & $-0.11^{\circ} \mathrm{yr}^{-1}$ \\
NPO NBL & upper $381 \mathrm{~m}$ & $1950-2008$ & from $15.5^{\circ}$ to $13.9^{\circ} \mathrm{N}$ & $-0.028^{\circ} \mathrm{yr}^{-1}$ \\
SPO SBL & upper $200 \mathrm{~m}$ & $1950-2010$ & from $14.5^{\circ}$ to $15.7^{\circ} \mathrm{S}$ & $-0.020^{\circ} \mathrm{yr}^{-1}$ \\
\hline
\end{tabular}

*See Figure 4 from Hu et al. [2015] for instance, where both Pacific NBL and SBL time series from these studies are displayed. $\mathrm{SAO}=$ South Atlantic Ocean, $\mathrm{NPO}=$ North Pacific Ocean, $\mathrm{SPO}=$ South Pacific Ocean. 


\title{
Supplementary Materials for
}

\author{
South Atlantic Surface Boundary Current System during \\ the Last Millennium in the CESM-LME: The Medieval \\ Climate Anomaly and Little Ice Age
}

Fernanda Marcello *, Ilana Wainer, Peter R. Gent,

Bette L. Otto-Bliesner, Esther C. Brady

* Correspondence: fernanda.marcello@usp.br

Figure S1 shows the model-derived LM-mean zonal velocity field compared to the literature (i.e., Figure 2 from Stramma and England, 1999 [1]).
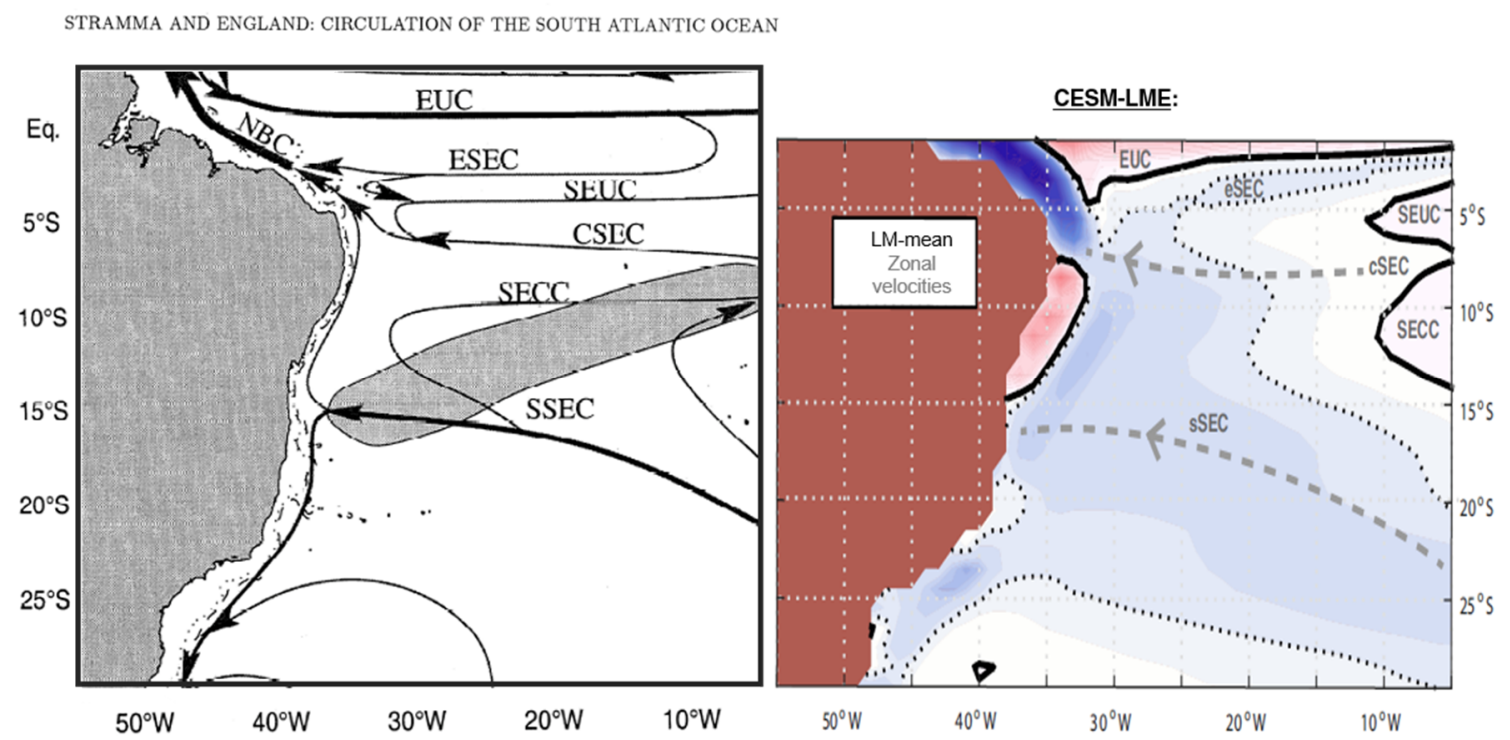

Figure S1. Scaled comparison of Figure 2 from Stramma and England (1999) [1] with Figure 2b of the original manuscript. Left: Schematic representation of the large-scale, upper 100-m geostrophic currents based on observations from the World Ocean Circulation Experiment (WOCE). Right: Depth integrated mean zonal velocities (m/s) over the top $200 \mathrm{~m}$ from the CESM-LME simulation results.

Figure S2 compares the CESM model results with those of five ocean reanalysis products: the European Center for Medium-Range Weather Forecasts (ECMWF) ocean analysis/reanalysis system 4 (ORAS4), the Geophysical Fluid Dynamics Laboratory (GFDL), the Simple Ocean Data Assimilation version 2.1.6 (SODA216), the Global Ocean Data Assimilation System (GODAS) and the Climate 
Forecast System Reanalysis (CFSR) from the National Centers for Environmental Prediction (NCEP) (Table S1).

Table S1. Time period, horizontal resolution and number of vertical layers above $1000 \mathrm{~m}$ of the 5 ocean reanalysis products.

\begin{tabular}{cccc}
\hline Product & Period & Horiz. Resolution & Vertical Layers-1000 m \\
\hline ORAS4 & $1960-2010$ & $1^{\circ} \times 1^{\circ}$ & 26 \\
\hline GFDL & $1961-2010$ & $1^{\circ} \times-^{*}$ & 34 \\
\hline SODA216 & $1960-2008$ & $0.5^{\circ} \times 0.5^{\circ}$ & 22 \\
\hline GODAS & $1980-2010$ & $1^{\circ} \times 1 / 3^{\circ}$ & 31 \\
\hline CFSR & $1980-2010$ & $0.5^{\circ} \times 0.5^{\circ}$ & 31 \\
\hline
\end{tabular}

* The latitudinal resolution of the GFDL reanalysis varies between approximately $0.33^{\circ}$ near the equator to $1^{\circ}$ near $30^{\circ} \mathrm{S}$.

The comparisons are made based on the periods covered by the reanalysis products, displayed in Table S1; on the 1948-2015 period for the ocean component of the CESM, the Parallel Ocean Program version 2 (CESM1-POP2, here as CESM-OCN) and on the interval of 1960-2010 for the CESM-LME.

The results are also quantitatively compared to those of Rodrigues et al. (2007) [2] (hereinafter R2007), who characterized the SSEC bifurcation vertical structure at the western boundary from hydrographic observations and numerical model results, providing a description of its annual mean depth dependence. The authors showed that the bifurcation occurs at about $10^{\circ}-14^{\circ} \mathrm{S}$ in the top 100 $\mathrm{m}$ and shifts poleward with increasing depth, reaching $27^{\circ} \mathrm{S}$ at $1000 \mathrm{~m}$ (Figure 3 in their manuscript).

Among the reanalysis products that were examined, the structure that more closely resembles the contour from R2007 hydrographic observations is the one reproduced by ORAS4. A previous investigation which evaluated the products' performance in terms of the mean circulation field for different levels in comparison to the available observations of R2007 (not shown), also suggested that ORAS4 better captures the dynamics of the SBL.

The mean SBL for individual levels up to $600 \mathrm{~m}$ is listed in Table S2. Despite some small differences, all data-sets reproduce the poleward tilting of the sSEC bifurcation with increasing depth. The total shift up to $600 \mathrm{~m}$ of the R2007 observations and of ORAS4, CESM-OCN and CESMLME are within the range of $9^{\circ}-13.1^{\circ}$.

Table S2. SBL values obtained by R2007 from hydrographic observations; derived from ocean reanalysis products and obtained from the CESM-models results.

\begin{tabular}{ccccccc}
\hline & Surface & $\mathbf{1 0 0} \mathrm{m}$ & $\mathbf{2 0 0} \mathrm{m}$ & $\mathbf{4 0 0} \mathrm{m}$ & $\mathbf{6 0 0} \mathrm{m}$ & Total shift \\
\hline Obs. $^{*}$ & $14^{\circ} \mathrm{S}$ & $14^{\circ} \mathrm{S}$ & $18.6^{\circ} \mathrm{S}$ & $21^{\circ} \mathrm{S}$ & $23.6^{\circ} \mathrm{S}$ & $9.6^{\circ}$ \\
\hline ORAS4 & $13^{\circ} \mathrm{S}$ & $19.6^{\circ} \mathrm{S}$ & $21.3^{\circ} \mathrm{S}$ & $23.3^{\circ} \mathrm{S}$ & $24.7^{\circ} \mathrm{S}$ & $11.7^{\circ}$ \\
\hline GFDL & $18.3^{\circ} \mathrm{S}$ & $20.1^{\circ} \mathrm{S}$ & $21.2^{\circ} \mathrm{S}$ & $25.5^{\circ} \mathrm{S}$ & $27.1^{\circ} \mathrm{S}$ & $8.8^{\circ}$ \\
\hline SODA216 & $13^{\circ} \mathrm{S}$ & $18.1^{\circ} \mathrm{S}$ & $22.1^{\circ} \mathrm{S}$ & $25.1^{\circ} \mathrm{S}$ & $27.6^{\circ} \mathrm{S}$ & $14.6^{\circ}$ \\
\hline GODAS & $7.2^{\circ} \mathrm{S}$ & $16.9^{\circ} \mathrm{S}$ & $19^{\circ} \mathrm{S}$ & $22.2^{\circ} \mathrm{S}$ & $24.1^{\circ} \mathrm{S}$ & $16.9^{\circ}$ \\
\hline CFSR & $8.4^{\circ} \mathrm{S}$ & $14.4^{\circ} \mathrm{S}$ & $16.5^{\circ} \mathrm{S}$ & $20.5^{\circ} \mathrm{S}$ & $22.6^{\circ} \mathrm{S}$ & $14.2^{\circ}$ \\
\hline CESM-OCN & $14.7^{\circ} \mathrm{S}$ & $19^{\circ} \mathrm{S}$ & $23.3^{\circ} \mathrm{S}$ & $25.7^{\circ} \mathrm{S}$ & $27.8^{\circ} \mathrm{S}$ & $13.1^{\circ}$ \\
\hline CESM-LME & $17.8^{\circ} \mathrm{S}$ & $20.3^{\circ} \mathrm{S}$ & $22.3^{\circ} \mathrm{S}$ & $24.9^{\circ} \mathrm{S}$ & $26.8^{\circ} \mathrm{S}$ & $9^{\circ}$ \\
\hline
\end{tabular}

* Hydrographic observations from R2007: geostrophic velocities determined from dynamic heights relative to 1000 dbar, calculated with an annual mean climatology of temperature and salinity constructed from observations (quality-controlled CTD and bottle data obtained from HydroBase (Curry, 1996) [3]). The authors' calculations do not include the Ekman current, which would affect the bifurcation latitude near the surface. They clarify that adding the Ekman currents to the geostrophic currents (calculated from observations) moves the bifurcation latitude northward by about $1^{\circ}$ (i.e., the bifurcation occurs at $13^{\circ}$ at the surface). 
Therefore, the simulation results from CESM-OCN and CESM-LME are similar to the results derived from ORAS4 and to R2007 hydrographic observations, suggesting that the model simulates well the sSEC bifurcation and the region of study.

These results also agree with the available literature. From hydrographic data, Stramma and England (1999) [1] showed that the bifurcation latitude is $16^{\circ} \mathrm{S}$ in the near-surface layer (top $100 \mathrm{~m}$ ), $20^{\circ} \mathrm{S}$ in the South Atlantic Central Water (SACW) layer (100-500 m), and $26^{\circ} \mathrm{S}$ in the intermediate layer (500-1200 m). Using isobaric RAFOS oats, Boebel et al. (1999) [4] showed that the Return Current (analog to the SEC, but within the Antarctic Intermediate Water (AAIW) layer) reaches the South American coast at approximately $28^{\circ} \mathrm{S}$ (called the Santos Bifurcation by the authors). Using data from the World Ocean Circulation Experiment (WOCE) hydrographic section A17, which was taken during the austral summer of 1994, Wienders et al. (2000) [5] estimated the transport of the SEC and its bifurcation latitude for several isopycnal layers: the SEC bifurcation latitude is $14^{\circ} \mathrm{S}$ at the surface, $24^{\circ} \mathrm{S}$ in the $26.7-26.9$ layer $(400-500 \mathrm{~m})$, and nearly constant around $26^{\circ}-28^{\circ} \mathrm{S}$ in the AAIW and Upper Circumpolar Water (UCPW: 600-1200 m). These results should be interpreted with caution because they are based on a single hydrographic section taken from $6^{\circ}-10^{\circ}$ from the western boundary. In the simulations by Harper (2000) [6], the bifurcation point in the near-surface layer at the western boundary of the SAO occurs at $18^{\circ} \mathrm{S}$, and in those by Malanotte-Rizzoli et al. (2000) [7], it occurs at $17^{\circ} \mathrm{S}$. From the results of two high-resolution ocean global circulation models (OGCMs), the Hybrid Coordinate Ocean Model-HYCOM and the Ocean Circulation and Climate Advanced Modeling Project-OCCAM, Pereira et al. (2014) [8] found that the latitude of bifurcation of the zonal ows reaching the coast (analog to the SEC), is $13^{\circ}-15^{\circ} \mathrm{S}$ for the Tropical Water, $22^{\circ} \mathrm{S}$ for the Central Water, $28^{\circ}-30^{\circ} \mathrm{S}$ for the Antarctic Intermediate Water. Cirano et al. (2006) [9], using data from the global circulation model Ocean Circulation and Climate Advanced Modeling Project (OCCAM), found that the bifurcation occurs between $9^{\circ}-15^{\circ} \mathrm{S}$ in the TW $(0-116 \mathrm{~m})$, migrating to $25^{\circ} \mathrm{S}$ in the SACW $(116-657 \mathrm{~m})$ and $25^{\circ}-30^{\circ} \mathrm{S}$ in the AAIW (657-1234 $\left.\mathrm{m}\right)$.

(a) ORAS4

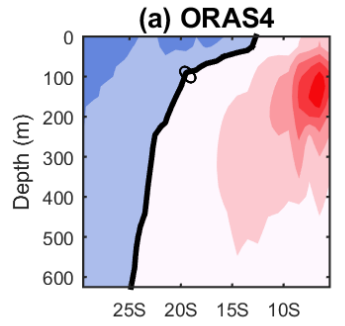

(d) GODAS

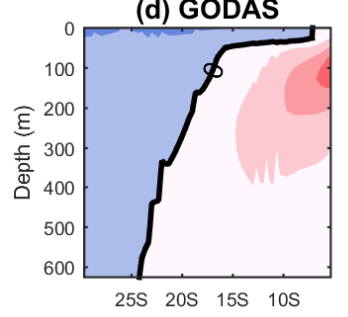

(g) CESM-LME

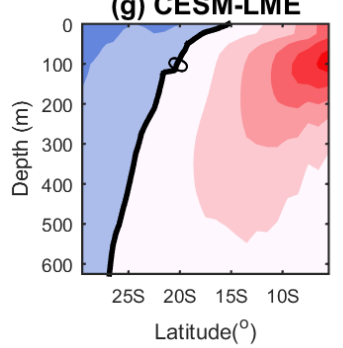

(b) GFDL

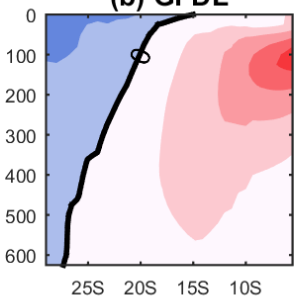

(e) CFSR
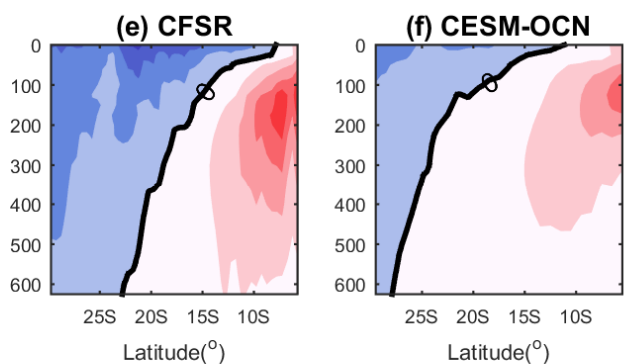

(c) SODA216

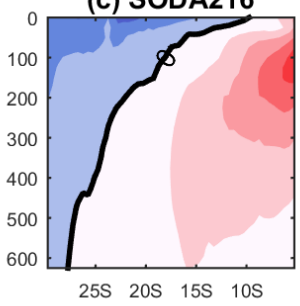

Latitude $\left({ }^{\circ}\right)$

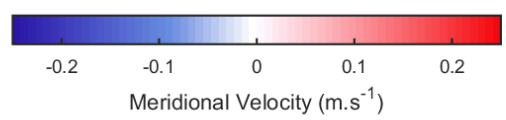

Figure S2. sSEC bifurcation vertical profile from reanalysis products and model results. Mean meridional velocity $(\mathrm{m} / \mathrm{s})$ averaged over the western boundary layer $\left(<4^{\circ}\right.$ from the coast) from ocean reanalysis products $(\mathbf{a}-\mathbf{e})$ and from model results $(\mathbf{f}-\mathbf{g})$. Positive (negative) values indicate northward 
(southward) ow associated with the NBUC (BC), and the contour of zero velocity represents the bifurcation of the sSEC.

\section{References}

1. Stramma, L.; England, M.H. On the water masses and mean circulation of the South Atlantic Ocean. J. Geophys. Res. Ocean. 1999, 104, 20863-20883.

2. Rodrigues, R.R.; Rothstein, L.M.; Wimbush, M. Seasonal Variability of the South Equatorial Current Bifurcation in the Atlantic Ocean: A Numerical Study. J. Phys. Oceanogr. 2007, 37, 16-30. doi:10.1175/JPO2983.1.

3. Curry, R.G. Hydrobase: A Database of Hydrographic Stations and Tools for Climatological Analysis; Technical Report; Woods Hole Oceanographic Institute: Woods Hole, MA, USA, 1996; WHOI-96-01, p. 50.

4. Boebel, O.; Davis, R.E.; Ollitrault, M.; Peterson, R.G.; Richardson, P.L.; Schmid, C.; Zenk, W. The Intermediate Depth Circulation of the Western South Atlantic. Geophys. Res. Lett. 1999, 26, 3329-3332.

5. Wienders, N.; Arhan, M.; Mercier, H. Circulation at the western boundary of the South and Equatorial Atlantic: Exchanges with the ocean interior. J. Mar. Res. 2000, 58, 1007-1039.

6. Harper, S. Thermocline ventilation and pathways of tropical-subtropical water mass exchange. Tellus $A$ Dyn. Meteorol. Oceanol. 2000, 52, 330-345.

7. Malanotte-Rizzoli, P.; Hedstromb, K.; Arango, H.; Haidvogel, D.B. Water mass pathways between the subtropical and tropical ocean in a climatological simulation of the North Atlantic ocean circulation. Dyn. Atmos. Oceans 2000, 32, 331-371.

8. Pereira, J.; Gabioux, M.; Marta-Almeida, M.; Cirano, M.; Paiva, A.M.; Aguiar, A.L. The Bifurcation of the Western Boundary Current System of the South Atlantic Ocean. Braz. J. Geophys. 2014, 32, 241-257.

9. Cirano, M.; Mata, M.M.; Campos, E.J.D.; Deiro, N.F.R. A Circulação Oceânica De Larga-Escala Na Região Oeste Do Atlântico Sul Com Base No Modelo De Circulação Global OCCAM. Revista Brasileira de Geofísica 2006, 24, 209-230.

(C) 2019 by the authors. Licensee MDPI, Basel, Switzerland. This article is an open access article distributed under the terms and conditions of the Creative Commons Attribution (CC BY) license (http://creativecommons.org/licenses/by/4.0/). 


\section{Highlights}

\section{1st Century Projected Changes in the Weddell Sea Antarctic Slope Current in a Downscaling Experiment}

Marina Noro, Marcos Tonelli, Fernanda Marcello, Bruno Ferrero, Ilana Wainer

- Potential feedbacks of the Antarctic Slope Current (ASC) to large-scale climate change are evaluated.

- External forcings regulates changes in the ASC frontal structure.

- Weakening of the ASC input in the Weddell Sea is driven by the decrease in the easterlies.

- In the outflow region, the strengthened ASC is followed by the shift to a Fresh Shelf regime. 


\title{
21st Century Projected Changes in the Weddell Sea Antarctic Slope Current in a Downscaling Experiment
}

\author{
Marina Noro ${ }^{\mathrm{a}, *}$, Marcos Tonelli ${ }^{\mathrm{a}}$, Fernanda Marcello ${ }^{\mathrm{a}}$, Bruno Ferrero ${ }^{\mathrm{a}}$, Ilana \\ Wainer $^{\mathrm{a}}$ \\ ${ }^{a}$ Department of Physical Oceanography, Oceanographic Institute of the University of São \\ Paulo, Praça do Oceanográfico, 191, Butantã, São Paulo, 05508-120, São Paulo, Brazil
}

\begin{abstract}
The Antarctic Slope Current (ASC) and its associated frontal structure regulate the exchanges between shelf and ocean waters. Changes in their structure and variability can alter the heat budget over the Antarctic margins and impact processes globally. In this study we investigate the ASC frontal structure response to climate change in the Weddell Sea region from a high-resolution experiment with the Regional Ocean Model System (ROMS) forced by MPIESM-MR simulation results for the CMIP5 RCP8.5 scenario (business as usual). Analysis were performed in two regions with distinct dynamics located in the inflow and outflow of the Weddell Sea. Results show regional variation in the external forcings (winds and sea ice) and, therefore, different responses along the ASC system to climate change. Weakening in the ASC volume transport driven mainly by the decrease in the easterlies magnitude is observed at the inflow region. The outflow shows strengthening trend due to enhanced lateral density gradient caused by freshening over the continental shelf. The outflow shifts from a Dense Shelf to a Fresh Shelf regime by the end of the 21st century. Changes in the ASC structure assessed by this study could be linked to accelerated sea ice melting and the reduction of the Antarctic Bottom Water formation.
\end{abstract}

Keywords: , Antarctic Slope Current, Antarctic Slope Front, High Resolution Regional Model, Climate Change, Weddell Sea

\footnotetext{
*Corresponding author

Email address: marina.noro.santos@gmail.com (Marina Noro )
} 


\section{Introduction} (Paolo et al., 2015).

The Antarctic Slope Current (ASC) consists of a westward transport current that encircles the Antarctic continental shelf and is associated with a frontal structure known as the Antarctic Slope Front (ASF). The ASF is defined by the resulting gradient between shelf and open-ocean waters (Jacobs, 1991; Heywood et al., 2004). The ASC regulates the water exchanged on and off the continental shelf (Jacobs, 1991; Thompson et al., 2018), connects processes occurring along the Antarctic coastal region and the global ocean. Its structure and variability can directly influence the basal melting of ice shelves and the formation of the Antarctic Bottom Water (AABW) (Thompson et al., 2018). The dense AABW ventilates a substantial fraction of the global abyssal ocean since it feeds the Meridional Overturning Circulation (MOC) lower limb, which plays an important role in Earth's climate by storing and transporting heat, fresh water, carbon and other properties through oceanic teleconnections (Rintoul et al., 2001; Marshall and Speer, 2012; Morrison et al., 2020). Therefore, the Antarctic coastal dynamics response to climate change could impact processes with global scales.

The study of the Antarctic coastal region is still a challenge. Besides the lack of observational data, climate models are not able to fully represent coastal dynamics. This requires higher spacial resolution to fully integrate the highly variable motions occurring at different scales in the Antarctic coastal environment, such as dense overflows, tides, and mesoscale eddies (Morrison et al., 2020; Flexas et al., 2015; Thompson et al., 2014; Hattermann et al., 2014; Nøst et al., 2011).

In addition to the complexity in integrating all the different scales of motion, there are regional differences controlling the ASC dynamics, which lead to different responses to climate change. The intensity and variability of the ASC control the onshore flow of Circumpolar Deep Water (CDW), the largest oceanic heat source for the Antarctic margins (Hattermann et al., 2014). Regions where the ASC is weak, such as the Bellingshausen and Amundsen seas, are more susceptible to warming due to the direct onshore transport of the CDW. In fact, the bottom shelf waters in both regions have warmed at a rate of $0.1^{\circ}$ to $0.3^{\circ} \mathrm{C}_{\text {decade }}^{-1}$ since the $1990 \mathrm{~s}$ (Schmidtko et al., 2014). Warmer water intrusions onto the continental margins drive basal melting, leading to a rapid loss of ice shelves. As a result, these regions are those which experienced greatest ice-shelf volume loss in the last decades 
The ice shelf loss reduces buttressing and accelerates glacial flow, which directly impacts sea level rise (Rignot et al., 2019; Schmidtko et al., 2014). From 1961 to 2004 the Antarctic glacial flow contributed by $28 \%$ of the global sea level rise and its contribution is likely to rise (Hock et al., 2009). Rignot et al. (2019) showed that from 1979 to 2017 the total ice sheet mass loss increased over these four decades gradually from $40 \pm 9 \mathrm{Gt} \cdot \mathrm{yr}^{-1}$ in the first decade (1979-1990) to $252 \pm 26 \mathrm{Gt} \cdot \mathrm{yr}^{-1}$ in 2009-2017. This mass loss had a contribution of $3.6 \pm 0.5 \mathrm{~mm}$ per decade to sea-level rise with a cumulative $14.0 \pm 2.0 \mathrm{~mm}$ since 1979 .

In addition to the contribution to sea level rise, the ice loss also increases freshwater flux into the Antarctic coastal seas, which modifies the Dense Shelf Water (DSW) properties and, consequently, the AABW formation (Moorman et al., 2020). Some studies (Purkey and Johnson, 2010, 2012) already show substantial warming and decrease of the volume of the AABW. Highresolution experiments also suggest that the enhanced coastal freshening by meltwater source and, consequently buoyant DSW, could lead to a slowdown or even the collapse of the AABW formation (Moorman et al., 2020).

Since the Weddell Sea is the main source region of the AABW (Carmack, 1974; Fahrbach et al., 1995), contributing with approximately $70 \%$ of its production (Orsi et al., 1999), and that the ASC system could have an impact on the AABW formation, this study investigates the changes in the ASC frontal structure in a climate change scenario. This is achieved with a regional ocean model downscaling experiment for the period of 2020 to 2100 . The changes are considered in view of the differences between the initial and final sub-periods: 2020:2029 and 2091:2100.

This work is organized as follows. Section 2 describes the Regional Ocean Model Downscaling Experiment results which were analyzed. Section 3 presents the results and associated discussions relative to volume transport changes, external forcings, regional hydrography, and trends for the 21st century. Section 4 finishes with the conclusions and final considerations.

\section{Donwscaling Experiment}

In order to investigate the global climate impacts on a regional-to-local scale along the Weddell Sea coastal region, we adopt a downscaling approach using the Regional Ocean Model System (ROMS) forced by the Max-PlanckInstitute Earth System Model Mixed Resolution (MPI-ESM-MR) (Jungclaus et al., 2013) simulation results from the Coupled Model Intercomparison 
Project phase 5 (CMIP5) for the Representative Concentration Pathway 8.5 (RCP8.5) scenario (business as usual).

The Regional Ocean Model System is a free-surface, terrain-following, hydrostatic primitive equations ocean model (Shchepetkin, 2003; Shchepetkin and McWilliams, 2005). All 2-D and 3-D equations are time-discretised using a third-order accurate predictor (Leap-Frog) and corrector (Adams-Molton) time-stepping algorithm. In the horizontal, the primitive equations are evaluated using boundary-fitted, orthogonal curvilinear coordinates on a staggered Arakawa-C grid. In the vertical, the primitive equations are discretised over variable topography using stretched terrain-following coordinates (Shchepetkin and McWilliams, 2005). More detailed descriptions may be found in Budgell (2005) and Wilkin and Hedstrom (1998).

For the model physical consistency and climatic stability, a dynamicthermodynamic sea-ice module is coupled to the ocean model, having both of them the same grid (Arakawa-C) and time step, and sharing the same parallel coding structure (Budgell, 2005).

The regional modeling domain used in the present study comprises the South Atlantic and its Southern Ocean sector. The north, west and east boundaries are open and forced by the climate output simulations outputs from the MPI-ESM-MR. The domain is limited to the south by the Antarctic Continent. The western boundary is located at the Drake Passage which receives the signal from the Antarctic Circumpolar Current (ACC) eastward flow, which is the most significant dynamic feature of the Southern Ocean (SO). The eastern boundary is located between Africa and Antarctica, allowing the outflow of the ACC as well as the dynamic input from the Agulhas Leakage. The northern boundary captures the Atlantic Meridional Overturning Circulation from the MPI-ESM-MR slightly northward of the Equator.

The domain consists of a variable curvilinear horizontal resolution grid designed to represent the oceanic dynamic features of the South Atlantic. The domain has $730 \times 514$ horizontal grid cells and 37 terrain-following vertical levels (sigma coordinates) with higher resolution near the surface and bottom. The ocean bottom topography is constructed combining ETOPO5 (National Geophysical Data Center - NGDC, 1988) and BEDMAP (in which sea floor topography is derived from the Smith and Sandwell (1997)), ETOPO2 and ETOPO5 (NGDC, 1988).

Higher resolution is found over the Weddell Sea domain, varying between $\approx 6 \mathrm{~km}$, near the coast to $\approx 11 \mathrm{~km}$ in the northern boundary. Spatial and temporal resolutions were calculated with the commitment between 
the mesoscale (eddy resolving) resolution and numerical performance for long climate (secular) integrations. Although the eddy parameterizations are considered in this model, this resolution is insufficient to fully resolve the ASF mesoscale eddy field, where the Rossby radius is $\approx 4 \mathrm{~km}$ and would require a horizontal grid of $\approx 1 \mathrm{~km}$ (Nøst et al., 2011; Hattermann et al., 2014; Thompson et al., 2014). Furthermore, it is worth mentioning that the model does not include tides, which also play an important role in cross-slope exchanges (Thompson et al., 2018).

The model has been tested for present day conditions using NOAA National Center for Environmental Prediction/National Centre for Atmospheric Research Reanalysis 2 (NCEP/NCAR R2) and Simple Ocean Data Assimilation Version 3.4.1 (SODA3.4.1). It was validated against data from different sources: the World Ocean Altas 2013 (WOA13), Archiving, Validation and Interpretation of Satellite Oceanographic data (AVISO), NOAA Extended Reconstruction SSTs Version 4 (ERSSTv4) (see Figure A.1). Despite its limitations, the model was to able to capture important Antarctic coastal features such as the AABW formation.

All the simulation results presented in this study are statistically significant at the $95 \%$ confidence level. Referred "significant changes", therefore, fall within this confidence level.

\section{Results and Discussion}

\subsection{Changes in Volume Transport}

Simulation results for the RCP8.5 scenario shows significant differences between the first (2020 to 2029) and the last decade (2091 to 2100) in the Weddell Sea full-depth integrated volume transport (Figure 1). In this scenario there is a weakening trend in almost the entire Weddell Sea volume transport. Exceptions are observed in the outflow along the Antarctic Peninsula, and in the eastern portion associated with an increasing trend in the ACC transport (north of our study area, not shown).

The strengthened transport along the ACC and in the eastern portion of the domain could indicate an enhanced CDW flow from the Weddell Gyre. This water mass mixes with colder waters above and below through its route within the Weddell Gyre, which alters its initial termohaline conditions, resulting in the cooler and fresher Warm Deep Water (WDW) (Garabato et al., 2002; Kerr et al., 2009). Since the WDW is transported westward along the southern branch of the gyre (Garabato et al., 2002), the effects enhanced 


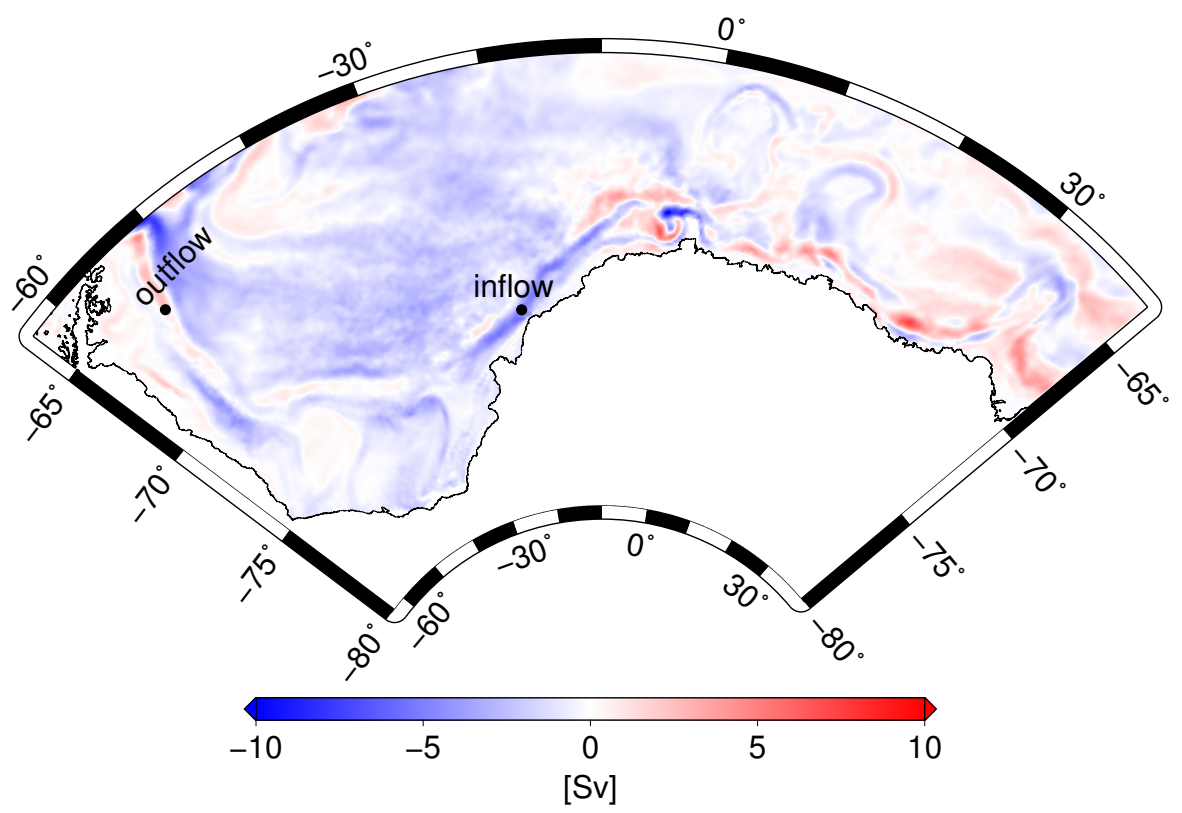

Figure 1: Climatological difference between the periods of 2091-2100 and 2020-2029 for the full-depth integrated volume transport. Difference values are significant at the $95 \%$ confidence level according to a t-test. Black dots illustrate the location of the inflow and outflow assessed regions.

heat content from WDW would be greater over the eastern continental shelf. On the other hand, the weakening in the inflow could increase the time lag for the the effects of warming on the eastern coast to be transported around the gyre and impact the outflow region.

To investigate changes along the ASC system and understand the mechanisms involved in both its weakening and strengthening, we selected two regions to perform our analysis (indicated in Figure 1). The first region is located at the inflow of the WS basin, where there is a significant decrease of $-4.86 \mathrm{~Sv}$ in the volume transport. The second location refers to the outflow, along the Antarctica Peninsula, where there is a small but significant increase of $1.24 \mathrm{~Sv}$.

\subsection{External Forcings}

The structure and variability of the ASC are controlled by both surface forcing (e.g. fluxes of momentum and buoyancy) and local environmental characteristics (e.g. bathymetry) (Thompson et al., 2018). Therefore, to 
assess the ASC's response to climate change, we also need to understand the behavior of its forcings in this scenario.

\subsubsection{Winds}

Previous studies have shown that winds play a major contribution to the ASC dynamics (Sverdrup, 1953; Fahrbach et al., 1992; Núñez-Riboni and Fahrbach, 2009; Stewart and Thompson, 2015). Prevailing easterly winds along the Antarctic coast induce coastal downwelling, establishing a subsurface meridional density gradient. For this reason, wind strength also modulates the frontal structure stability.

In our climate scenario there is a weakening trend in the wind magnitude south of $\approx 67^{\circ} \mathrm{S}$, whereas in northernmost latitudes it has strengthened (Figure $2 \mathrm{c}$ ). Greatest weakening is observed in the easterlies along the eastern Weddell Sea coastal region $\left(\approx 5^{\circ} \mathrm{W}-10^{\circ} \mathrm{E}\right)$, where it has decreased by $\approx 1.3$ $\mathrm{m} \cdot \mathrm{s}^{-1}$ in magnitude (Figure $2 \mathrm{c}$ ). Comparing the climatologies of the first (2020-2029; Figure2 a) and last decades (2091-2100; Figure2 b), we can also observe the poleward strengthening of the westerlies. Winds with magnitudes between $5 \mathrm{~m} \cdot \mathrm{s}^{-1}$ and $8 \mathrm{~m} \cdot \mathrm{s}^{-1}$, previously occurring in the latitude band of $\approx 50^{\circ}-60^{\circ} \mathrm{S}$ (Figure $\left.2 \mathrm{a}\right)$, are observed further south $\left(\approx 62^{\circ} \mathrm{S}\right)$ in the last decade (Figure 2 b). Moreover, in the Weddell Sea northern boundary ( $\approx 64^{\circ} \mathrm{S}$ between $\approx 60^{\circ} \mathrm{W}$ and $20^{\circ} \mathrm{W}$ ), there was an increase of $\approx 1.0 \mathrm{~m} \cdot \mathrm{s}^{-1}$ and the wind component has become more zonal.

These results suggest different responses to take place in the inflow and outflow regions. In the inflow, weaker coastal winds reduce the downward Ekman pumping near the coast and its associated meridional pressure gradient, which weakens the ASC and its frontal structure (Spence et al., 2014). Moreover, the along-slope component of the ASC can also be influenced by a remote response to changes promoted by an even greater decrease in the easterlies further east. As shown by Spence et al. (2017), wind perturbations can be propagated along the Antarctic coastline by coastal-trapped barotropic Kelvin waves, which can influence ASF and CDW transport along the Antarctic margin. On the other hand, the outflow is influenced by the strengthened westerlies. Although these winds can act to intensify the northward along-slope transport of the ASC along the Antarctica Peninsula, they can also contribute to reducing the Ekman pumping near the coast, impacting the cross-slope exchanges as well as in the inflow region.

The results here presented are consistent with the Southern Annular Mode (SAM) positive trend effects on the Southern Ocean, even though fur- 

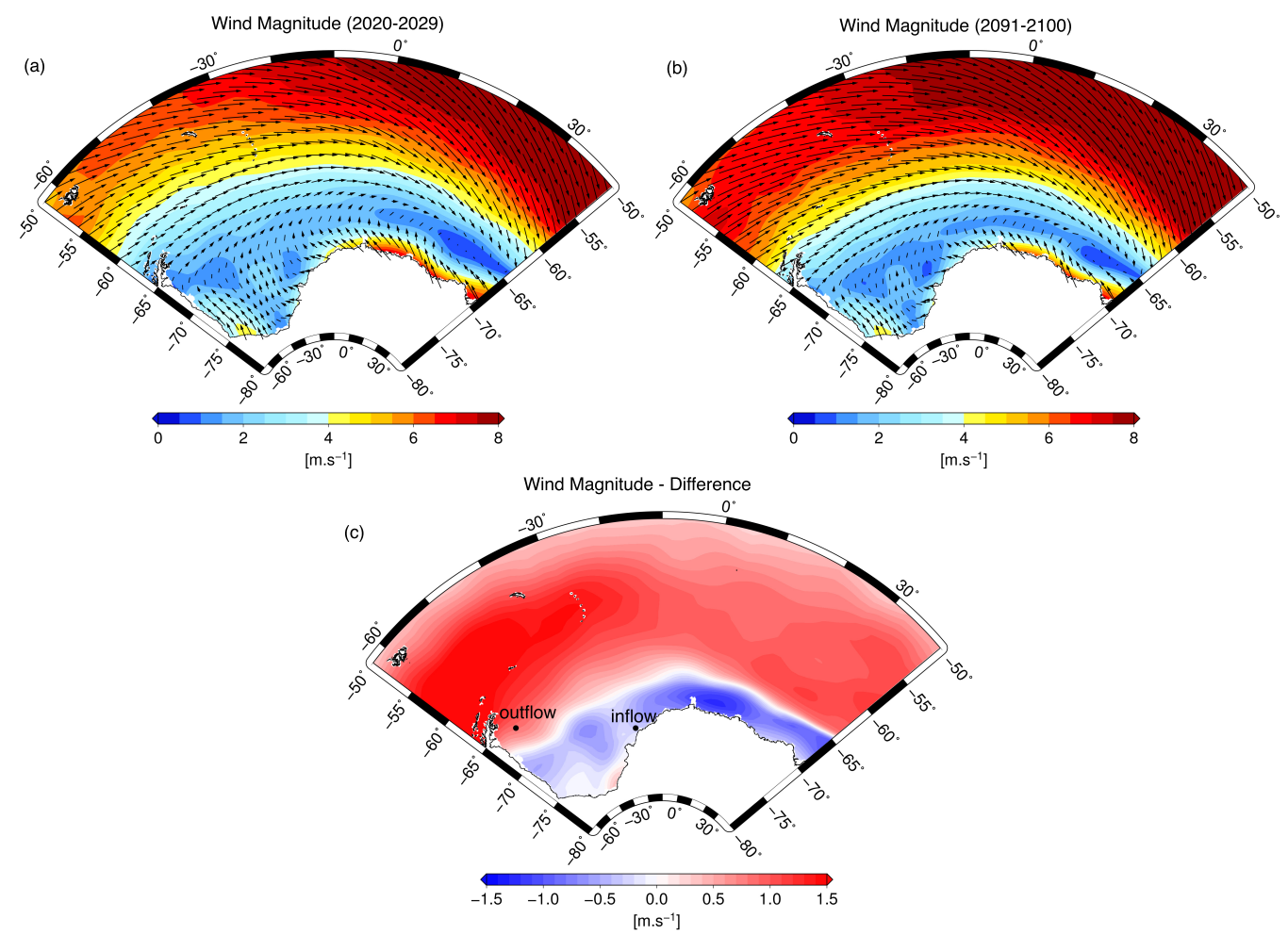

Figure 2: Wind magnitude averaged for the (a) first decade (2020 to 2029) and (b) last decade (2091 to 2100). (c) significant differences values at $95 \%$ between first (2020 to 2029 ) and last (2091 to 2100) decades determined with a t-test in each grid point. 
ther analysis are needed to evaluate the association with long-term trends. SAM is the dominant mode of atmospheric variability in the Southern Hemisphere extratropics, and a trend towards its positive phase has been observed since the mid-1960s (Thompson and Wallace, 2000; Kwok and Comiso, 2002; Marshall, 2003).

Meijers et al. (2016) showed that SAM positive phase is linked to stronger cyclonic wind stress curl over the Weddell Gyre, and has a strong correlation with the outflow from the Weddell Sea. This agrees with the increased transport in the WS outflow shown in Figure 1. In addition, changes in the wind pattern can also influence other features, such as the mesoscale eddy field, sea level patterns, and upwelling of CDW, which can significantly impact the ASC structure (Hogg et al., 2015; Frankcombe et al., 2013; Thompson et al., 2018).

\subsubsection{Sea Ice}

Despite the strong regional differences, from 1979 to 2015 the total Antarctic sea ice extent (SIE) increased at a small but significant rate of $\approx 1.7 \%$ $\operatorname{dec}^{-1}$ (Comiso et al., 2017; Turner et al., 2015). In the WS, SIE increased at a rate of $55 \times 10^{3} \mathrm{~km}^{2} \mathrm{dec}^{-1}$ from 1979 to 2013 (Turner et al., 2015). However, this increasing trend observed in the past three decades is not represented by climate models and, in fact, most of them have an SIE decrease (Turner et al., 2013). The reasons for the overall increase in Antarctic SIE are still under debate (Turner et al., 2013). However, after nearly three decades of observed increasing trends, the Antarctic sea ice had an exceptional retreat in 2016 springtime (Meehl et al., 2019; Turner et al., 2017) and the sea ice area decreased by $6.44 \times 10^{6} \mathrm{~km}^{2}$ (Turner et al., 2017). Although anomalous negative retreat occurred in all sectors, the Weddell region made the largest contribution (34\%) to this retreat (Turner et al., 2020, 2017).

Less sea ice allows more shortwave radiation to be absorbed in the early summer, resulting in an anomalous upper ocean warming which extended to the ocean mixed layer (OML) (Turner et al., 2020). During summer $2016 / 2017$ the SST in the WS reached a record high of $0.75^{\circ} \mathrm{C}$ and OML temperatures increased by $\approx 0.5^{\circ} \mathrm{C}$ (Turner et al., 2020). The establishment of the positive OML temperature anomaly contributed to the maintenance of the SIE anomalies which has persisted for the following three summers (Turner et al., 2020). It marks an unprecedented change in Weddell Sea sea ice, but it is still soon to evaluate if it represents the start of a longer-term decline as represented by numerical models (Turner et al., 2020). 
Although the representation of Antarctic sea ice by numerical models is still a challenge, significant advances have been made and are discussed by (Dinniman et al., 2016). This study uses a high resolution model, which was also previously used to represent high southern latitudes (Dinniman et al., 2012; Galton-Fenzi et al., 2012), however, deeper analysis (e.g. seasonal variability, melting rates) of the sea ice representation by our model falls beyond the scope of this paper. Here our aim is just to address the behavior of the sea ice in our numerical projection to discuss our results, since it is an important component for the coastal dynamics. Even considering the limitations, it could provide us some insights on how the coastal processes can be affected by a scenario of extreme sea ice loss like those seen in the past three summers.
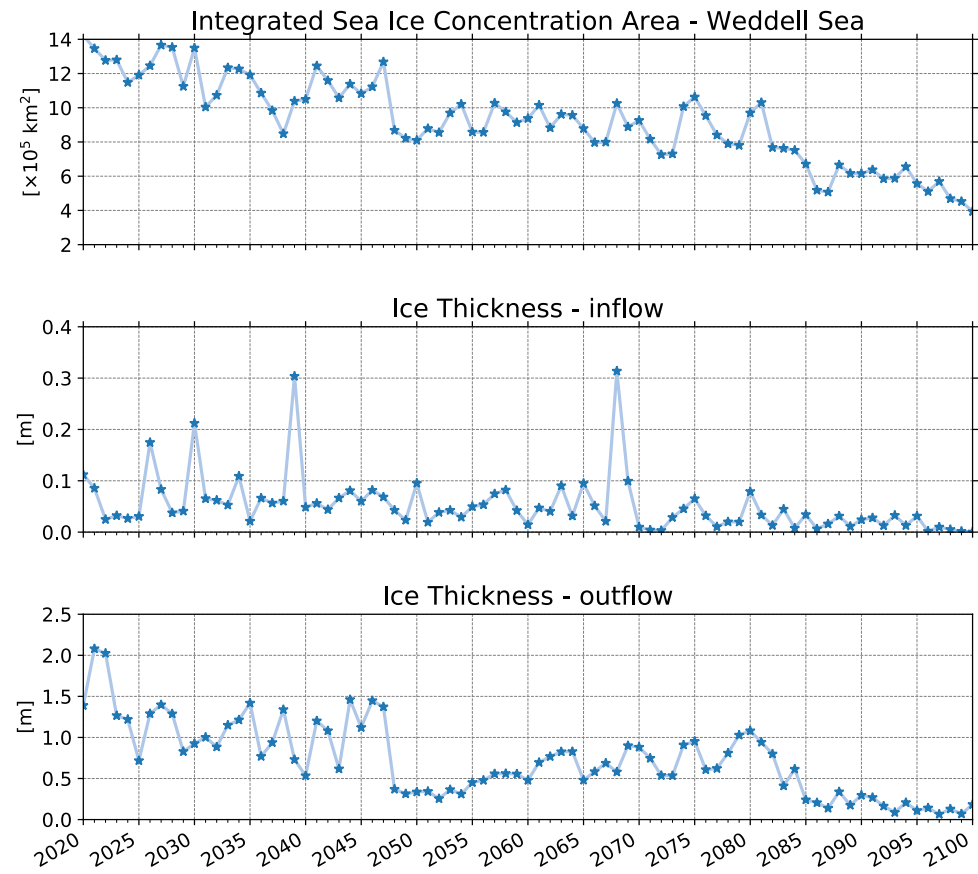

Figure 3: Annual mean time series of the (a) Weddell Sea sea ice concentration area (b) sea ice thickness in the inflow (c) sea ice thickness in the outflow.

In our simulation, there is an expressive sea ice loss in the WS, with a decrease of $\approx 1.4 \times 10^{6} \mathrm{~km}^{2}$ to $\approx 4.0 \times 10^{5} \mathrm{~km}^{2}$ in the annual mean concentration area (Figure $3 \mathrm{a}$ ), which represents a loss of $\approx 1.0 \times 10^{5} \mathrm{~km}^{2}$ from the beginning to the end of the century. Looking at the ice thickness 
in each region separately, there is a difference of up two orders between the inflow and the outflow. The inflow (Figure $3 \mathrm{~b}$ ) shows small sea ice concentrations for the entire period simulated. In this region, there is a thin sea ice layer and the annual-mean thickness remains below $0.10 \mathrm{~m}$. In only six of the eighty-one years simulated it reaches values above that, the maximum being $0.31 \mathrm{~m}$ in 2069. After 2080 the sea ice thickness remains below 0.05 $\mathrm{m}$ and decreases to a minimum of $2.67 \times 10^{-4} \mathrm{~m}$ at the end of the century. The outflow (Figure $3 \mathrm{c}$ ), in turn, shows greater variation during the period simulated. From 2020 to 2047 the sea ice thickness remains mostly above 1.0 $\mathrm{m}$, and reaches a maximum value of $2.08 \mathrm{~m}$ at the beginning of the century. After this there is a sharp decrease to $\approx 0.3 \mathrm{~m}$ between 2048 and 2054 . From 2055 to 2080 the sea ice thickness increases again and varies between $0.45 \mathrm{~m}$ and $1.08 \mathrm{~m}$ within this period. From 2081 a new period of decline begins, and from 2085 to 2100 it reaches minimum levels that vary between $0.06 \mathrm{~m}$ and $0.34 \mathrm{~m}$.

\subsection{Hydrography and ASC frontal system \\ 3.3.1. Characterization of the mean climatological fields}

As proposed by Thompson et al. (2018), the inflow can be classified as a Fresh Shelf regime (Figure $4 \mathrm{a}$ ), characterized by density surfaces that tilt downward and poleward from the surface towards the continental slope, marked by a half 'V' shape (Jacobs, 1991; Thompson et al., 2018). Here the density layers associated with WDW do not extend onto the shelf and intersect the continental slope. Considering the upper limit of the WDW density $\left(27.36 \mathrm{~kg} / \mathrm{m}^{3}\right.$ in our results, Figure 4 a) and the $0^{\circ} \mathrm{C}$ isotherm (Figure $4 \mathrm{c}$ ), the WDW intersects the continental slope at approximately $680 \mathrm{~m}$ depth in the climatology for the entire period analyzed (2020 to 2100). In this region the persistent and strong easterly winds induce the coastal downwelling and a westward flowing ASC, establishing a strong front that separates continental shelf cold and fresh water from warm and salty WDW offshore (Jacobs, 1991; Spence et al., 2014; Thompson et al., 2018). Due to this strong front, there is weak cross-slope exchange, which limits the pathway of WDW onto the shelf (Thompson et al., 2018).

The outflow in turn, is classified as a Dense Shelf regime, which is marked by a V-shaped frontal structure (Figure $4 \mathrm{~b}$ ) that allows both an onshore transport of the WDW and export of the dense DSW (Thompson et al., 2018). In this case, isopycnals also tilt down towards the continental slope, but as they approach the shelf break they shoal again. In Dense Shelf regime 
regions the $\mathrm{V}$-shaped structure enables a large range of densities to have access to the continental shelf without intersecting frictional layers (Thompson et al., 2018). Despite the occurrence of surface densities associated with the WDW, the water remains cold due deep convection and dense water formation (Thompson et al., 2018).

Compared to observed temperature and salinity from previous studies (Orsi et al., 1993; Robertson et al., 2002), our climatologies (2020 to 2100) show a scenario of warmer (Figure $4 \mathrm{c}$ and $\mathrm{d}$ ) and fresher waters (Figure $4 \mathrm{e}$ and f). Despite these "lighter" water masses, the ASC frontal classification proposed by Thompson et al. (2018) is preserved in both regions.

\subsubsection{External forcings effects on the hydrographic fields}

Although the inflow sets up a region with a strong front, warmer waters are observed in the entire water column (Figure $4 \mathrm{c}$ ) compared to the outflow (Figure $4 \mathrm{~d}$ ). The decrease in temperature towards the outflow is a result of the mixing and heat distribution processes within the Weddell Gyre. However, changes in the external forcings, discussed in Section (3.2), suggest different hydrographic responses in each region analysed, which can impact the ASC frontal structure and dynamics in different ways.

The strengthening and southward shift of the westerlies lead to an enhanced inflow of the CDW into the Weddell Gyre, resulting in a warmer WDW and, therefore, a weaker front between the ACC and the northern limb of the gyre (Fahrbach et al., 2004). Since the eastern WS coastal region receives modified WDW, and considering a weakened ASF due to the decrease in the easterlies magnitude, the inflow region is also more impacted by a greater amount of heat.

Therefore, the scenario of small sea ice concentration, discussed in Section 3.2.2, could also be related with the WDW intrusion in the eastern WS. The thin sea ice cover in the inflow, allows greater shortwave radiation absorption and, therefore, warmer surface waters (Figure $4 \mathrm{c}$ ). In this region, waters below zero occur near the coast and in a thin layer offshore between $50 \mathrm{~m}$ and $150 \mathrm{~m}$ approximately, but they do not get colder than $-1.10^{\circ} \mathrm{C}$.

In the outflow, waters below $0^{\circ} \mathrm{C}$ cover the entire continental shelf and extend from the surface to $\approx 200 \mathrm{~m}$ depth offshore, reaching a minimum of $-1.57^{\circ} \mathrm{C}$. The outflow region is more susceptible to warming, since the WDW transport onto the shelf is favored by local dynamics, however, the WDW at the outflow is actually colder - reaching a maximum of $0.87^{\circ} \mathrm{C}$ (Figure 4 d) compared to $\approx 1^{\circ} \mathrm{C}$ at the inflow (Figure $4 \mathrm{c}$ ). This is due to the mixing 
a)

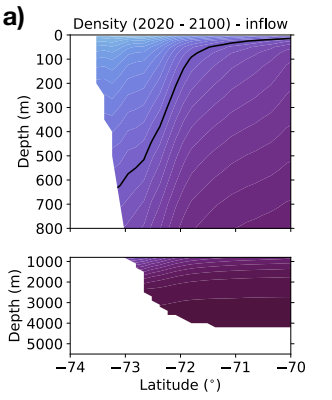

b)

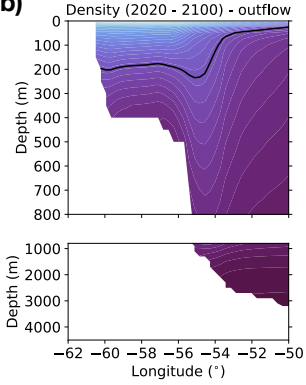

C) Temperature $(2020-2100)$ - inflow

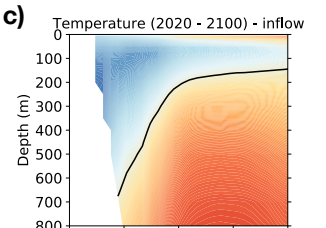

$\begin{array}{llr}27.30 & 27.45 & 27.60\end{array}$

d)

d) Temperature (2020 - 2100) - outflow
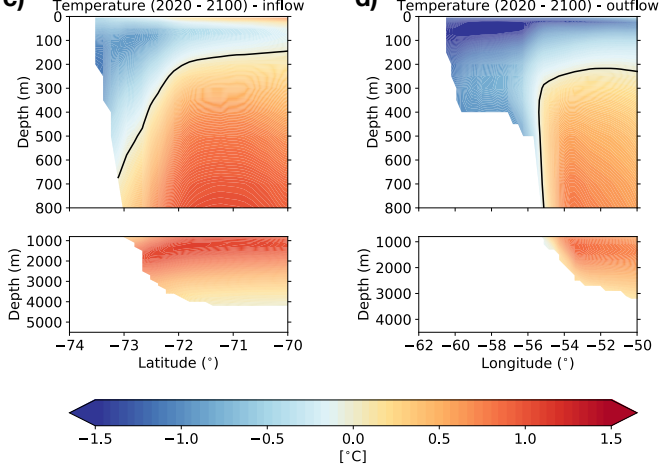

(a)

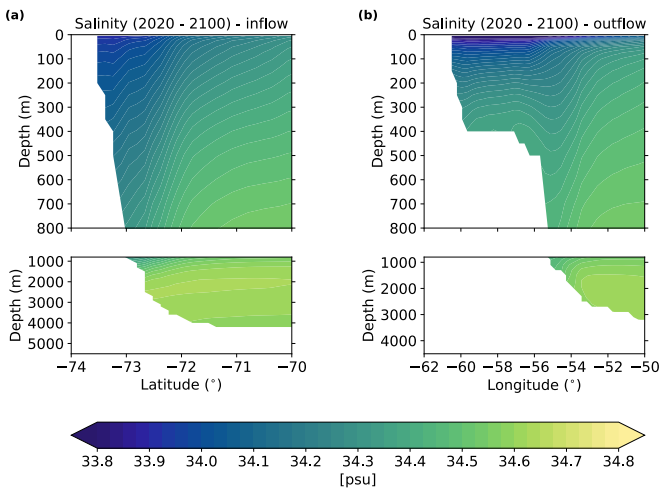

Figure 4: Density cross section climatologies for the period of 2020 to 2100 in the (a) inflow and (b) outflow. The black contour line marks the $\gamma=27.36 \mathrm{~kg} \cdot \mathrm{m}^{3}$ isopycnal in the inflow (a) and the $\gamma=27.39 \mathrm{~kg} \cdot \mathrm{m}^{3}$ in the outflow (b), corresponding to minimum density associated with WDW in each region.Temperature cross section climatologies for the period of 2020 to 2100 in the (c) inflow and (d) outflow. The black contour line marks the upper $0^{\circ} \mathrm{C}$ isotherm. Salinity cross section climatologies for the period of 2020 to 2100 in the (e) inflow and (f) outflow. 
with colder waters within the Weddell Gyre (Fahrbach et al., 2004, 2011). Moreover, the lowest salinity values are found in the outflow, within the minimum temperature layer over the shelf, with values below $34 \mathrm{psu}$ (Figure $4 \mathrm{f}$ ). This also illustrates the greater sea ice variability and its associated freshwater flux in this region.

Previous studies have shown a shoaling and warming trend of WDW since 1990's, although it has experienced some periods of cooling (Robertson et al., 2002; Fahrbach et al., 2004, 2011). This pattern in the WDW has also been reproduced by climate models (Tonelli et al., 2019). In our study, climatologies (Figure $4 \mathrm{c}$ and $\mathrm{d}$ ) show that the intermediate layer temperature is on average within the range of observations for the WDW (Whitworth et al., 1998; Orsi et al., 1993; Robertson et al., 2002). The salinity, in turn, shows fresher waters, ranging from 34.30 to 34.62 psu in both the inflow and the outflow (Figure $4 \mathrm{e}$ and $\mathrm{f}$ ). The maximum salinity reached by the simulated WDW, however, is near the minimum observed for this water mass (34.60) (Whitworth et al., 1998; Orsi et al., 1993; Robertson et al., 2002).

Our simulation also represents a thicker WDW layer. The upper boundary mean depth in offshore waters, determined by the $0^{\circ} \mathrm{C}$ isotherm, is $\approx$ $150 \mathrm{~m}$ in the inflow (Figure $4 \mathrm{c}$ ) and $\approx 210 \mathrm{~m}$ in the outflow (Figure 4 d). However, since the bottom layer shows waters with temperatures above $0^{\circ} \mathrm{C}$, to identify the WDW bottom layer we computed the WDW as the $40 \%$ warmest grid points $(>60$ th percentile of $\theta$ ). Considering this, we determined the density range of the simulated WDW.

Fahrbach et al. (2011) and Thompson et al. (2018) consider $\gamma^{n}=28.0$ $\mathrm{kg} / \mathrm{m}^{3}$ as the WDW neutral density limit. However, our projection yields a less dense WDW. In this scenario, the density surfaces associated with WDW varies between $27.36 \mathrm{~kg} / \mathrm{m}^{3}$ and $27.64 \mathrm{~kg} / \mathrm{m}^{3}$ in the inflow (Figure 4 a) and between $27.39 \mathrm{~kg} / \mathrm{m}^{3}$ and $27.64 \mathrm{~kg} / \mathrm{m}^{3}$ in the outflow (Figure $4 \mathrm{~b}$ ). Therefore, considering the $27.64 \mathrm{~kg} / \mathrm{m}^{3}$ isopycnal, the WDW layer reaches $3000 \mathrm{~m}$ depth, which is too deep for this water mass that typically ranges from $200 \mathrm{~m}$ to 150 0m (Schröder and Fahrbach, 1999; Kerr et al., 2009).

Fahrbach et al. (2011) have shown that a thicker WDW layer is linked to periods of warming, which is explained by increased inflow due to instabilities of the boundary of Weddell Gyre and the ACC (Fahrbach et al., 2004). Purkey and Johnson (2012) have shown that the bottom water contraction in the WS is compensated by an increase in the WDW volume, which deepened at a rate of $-8.1( \pm 4.5) \mathrm{m} \cdot \mathrm{s}^{-1}$ between the $1980 \mathrm{~s}$ and $2000 \mathrm{~s}$. In addition, the observed slowdown of the AABW production has showed to be 
consistent with the freshening in AABW formation sites, as the Weddell Sea (Hellmer et al., 2011; Purkey and Johnson, 2012). Thus, the strengthening and poleward shift of the westerlies (Figure 2), combined with other factors like sea ice melting, could impact the AABW production by means of the increased input of CDW into the WS, leading to a warmer and more voluminous WDW. However, it is essential to highlight that in the present study we do not quantify changes in the WDW volume as well as the impacts on the AABW formation.

\subsubsection{Trends and Changes in the 21st Century}

Our results show expressive changes in the intensity and geometry of the ASC frontal structure in both inflow and outflow regions (first rows of Figures 5 and 6 , respectively). These changes can significantly alter the ASC dynamics and exert a strong influence over the heat budget of the Antarctic margin (Thompson et al., 2018). Considering that, our simulation shows a less dense water column. Besides that, the comparison between the first (2020-2029) and last (2091-2100) decades indicates the decrease in densities throughout the simulation period in both regions (Figures $5 \mathrm{c}$ and $6 \mathrm{c}$ ).

In the inflow, this less dense water column is a contribution of both warming (Figure 5 second row) and freshening (Figure 5 third row). However, there is a different pattern between regions of greater increase in temperature and decrease in salinity across the domain. Less dense waters combined with the change in geometry of isopycnals, which has become less steep at the end of century (Figure $5 \mathrm{~b}$ ), allowed a larger range of densities to reach the continental shelf. The upper limit of WDW, determined by the $0^{\circ} \mathrm{C}$ isotherm, also shows shoaling at the slope. In the first decade the $0^{\circ} \mathrm{C}$ isotherm tilts downward and reaches the $750 \mathrm{~m}$ depth (Figure $5 \mathrm{~d}$ ), at the end of the century this isotherm is found at $400 \mathrm{~m}$ depth (Figure $5 \mathrm{e}$ ). As a result, the greatest warming occurs over the shelf break, where the water column warmed up to $1.10^{\circ} \mathrm{C}$ (Figure $5 \mathrm{f}$ ) subjected to the highest warming trend $\left(\approx 0.015^{\circ} \mathrm{C} \cdot \mathrm{yr}^{-1}\right.$; Figure 7 a).

Although the upper boundary of the WDW is shallower at the slope, in the waters offshore it remains at the same depth $(\approx 150 \mathrm{~m})$. However, this water mass undergoes warming and reaches a maximum temperature of $1.4^{\circ} \mathrm{C}$ in the climatology of the last decade (Figure $5 \mathrm{e}$ ). Differences between first and last decades are greater $\left(+0.75^{\circ} \mathrm{C}\right)$ only at a small portion northward (Figure $5 \mathrm{f}$ ), but this water mass layer shows significant warming of $\approx$ $0.012^{\circ} \mathrm{C} \cdot \mathrm{yr}^{-1}$ (Figure $7 \mathrm{a}$ ), which is consistent with observations (Robertson 
a)
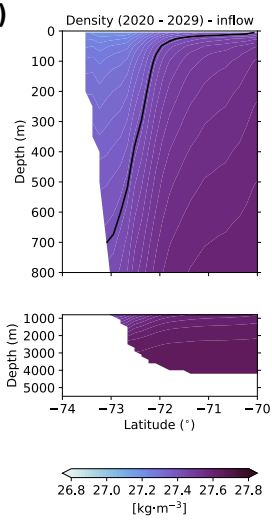

d)
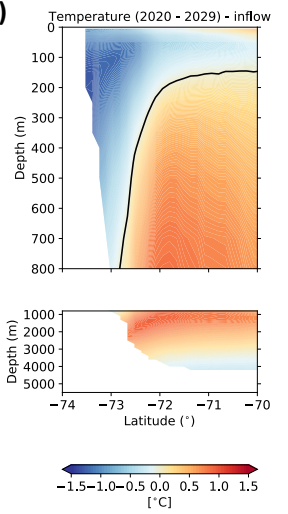

g)
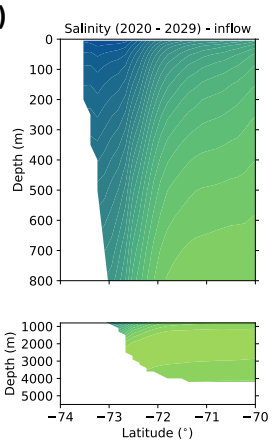

$\longdiv { 3 3 . 8 3 4 . 0 3 4 . 2 3 4 . 4 3 4 . 6 3 4 . 8 }$ b)
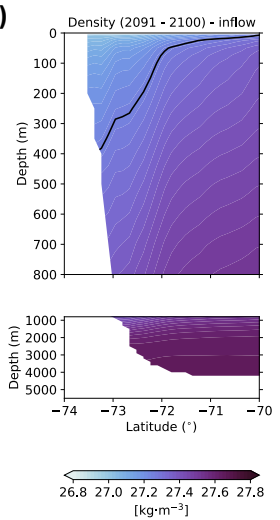

e)
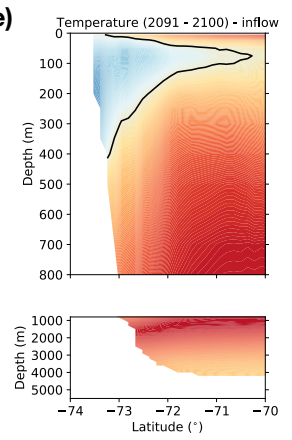

h)
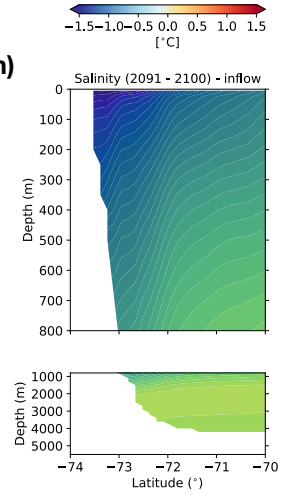

$\underset{33.8 \text { 34.0 } 34.234 .434 .634 .8}{[\mathrm{psu}]}$ c)
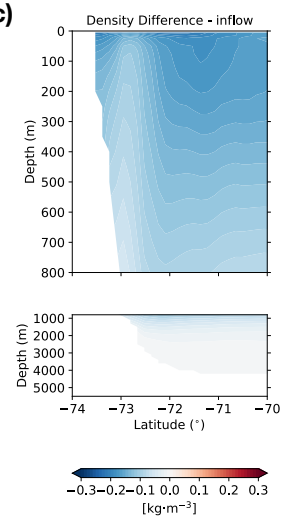

f)
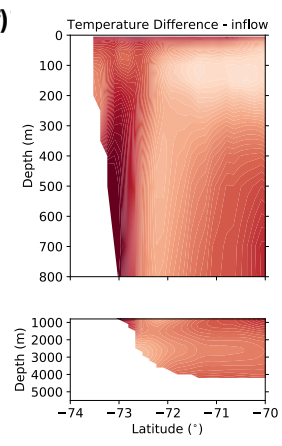

i)
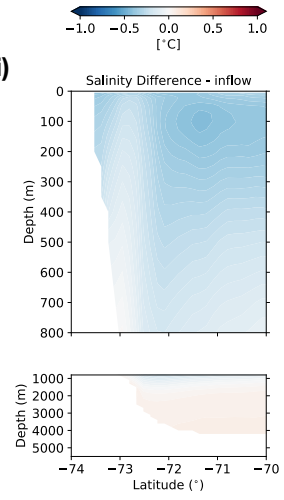

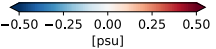

Figure 5: First row - density cross section averaged for the period the (a) first decade (2020 to 2029) and (b) last decade (2091 to 2100) in the inflow. (c) Significant differences values at $95 \%$ between first and last decades determined with a t-test in each grid point. The black contour marks the $\gamma=27.41 \mathrm{~kg} \cdot \mathrm{m}^{3}$ isopycnal in the first decade (a) and the $\gamma$ $=27.25 \mathrm{~kg} \cdot \mathrm{m}^{3}$ in the last decade (b), corresponding to minimum density associated with WDW in each decade. Second row - similar to the first row but for temperature. The black contour marks the upper $0^{\circ} \mathrm{C}$ isotherm. Third row - similar to first row for salinity. 
a)
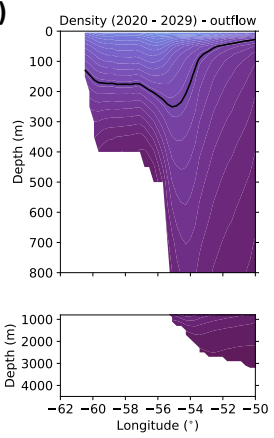

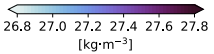

d)
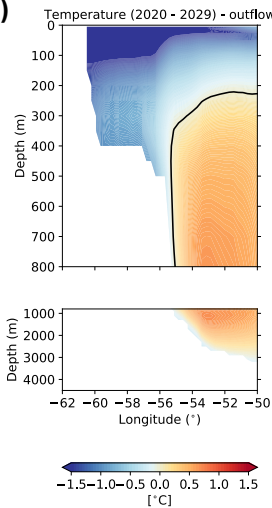

g)
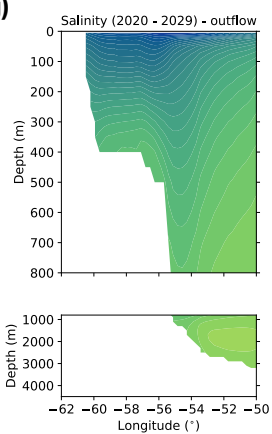

$\underset{33.834 .034 .234 .434 .634 .8}{\longrightarrow}$ b)
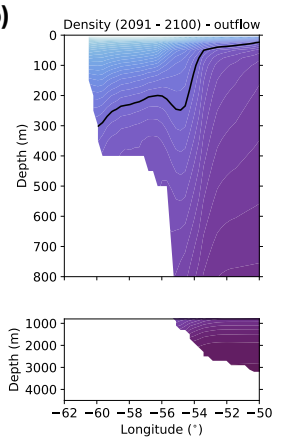

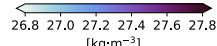

e)
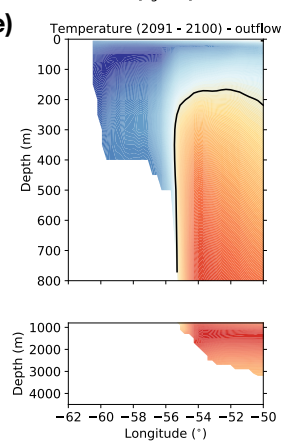

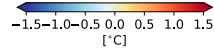

h)
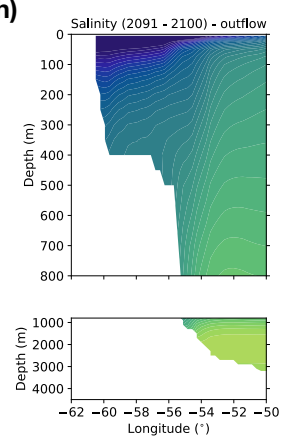

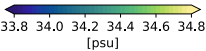

c)
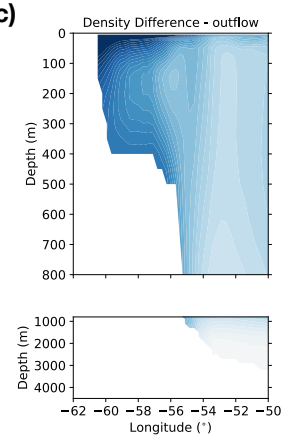

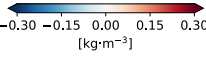

f)
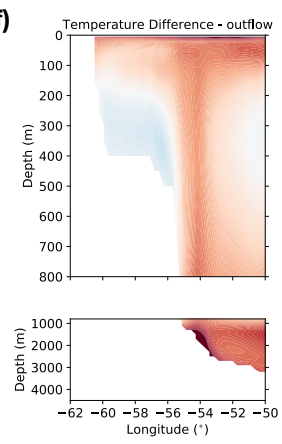

i)
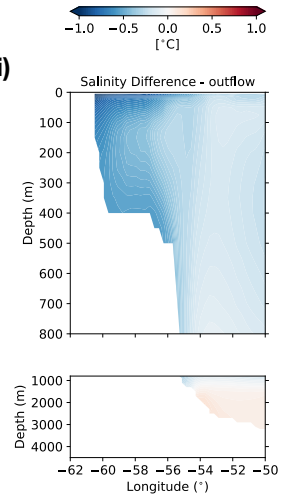

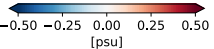

Figure 6: First row - density cross section averaged for the period the (a) first decade (2020 to 2029) and (b) last decade (2091 to 2100) in the outflow. (c) Significant differences values at $95 \%$ between first (2020 to 2029) and last (2091 to 2100) decades determined with a t-test in each grid point. The black contour marks the $\gamma=27.45 \mathrm{~kg} \cdot \mathrm{m}^{3}$ isopycnal in the first decade (a) and the $\gamma=27.32 \mathrm{~kg} \cdot \mathrm{m}^{3}$ in the last decade (b), corresponding to minimum density associated with WDW in each decade. Second row - similar to the first row for temperature. The black contour marks the upper $0^{\circ} \mathrm{C}$ isotherm. Third row - similar to first row for salinity. 
a)
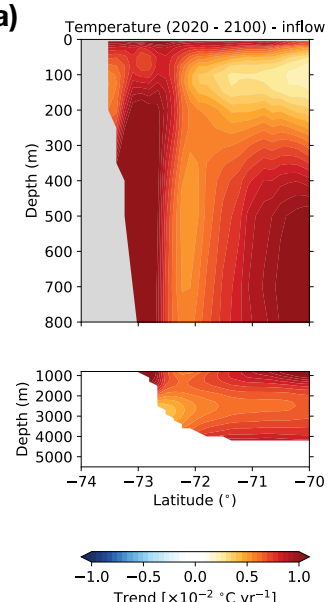

d)
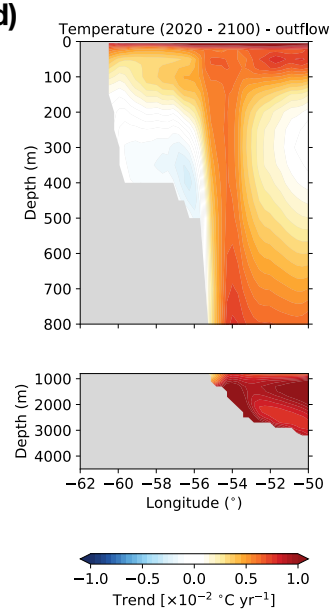

b)
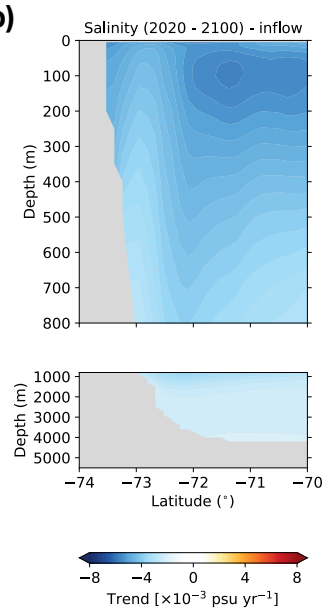

e)
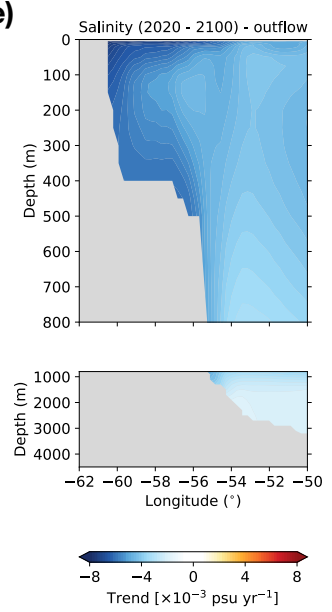

c)
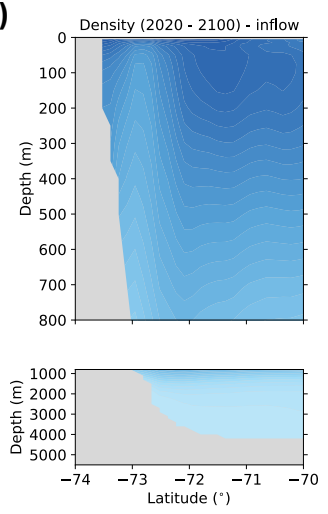

f)
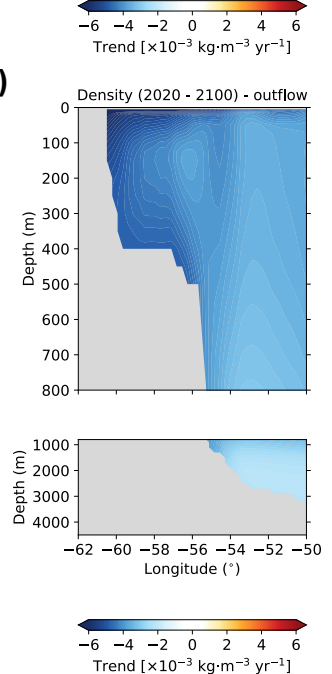

Figure 7: First row - trends in the inflow (a) Temperature, (b) Salinity, and (c) Density over the period of 2020 to 2100. Second row - trends in the outflow (d) Temperature, (e) Salinity, and (f) Density over the period of 2020 to 2100. Trends are significant at $95 \%$ level and were tested by Mann-Kendall test. 
et al., 2002). In addition, although the simulated WDW is found too deep in the first decade (Figure $5 \mathrm{~d}$ ), there is still a thin layer with temperatures below zero at the bottom, but it does not exceed $-0.33^{\circ} \mathrm{C}$.

As well as isopycnals, isohalines are also less steep over the last decade (Figure 5 e). Greater differences in salinity are concentrated at the surface layers offshore, where it decreases down to $-0.21 \mathrm{psu}$ from the first to the last decade (Figure $5 \mathrm{f}$ ). Over the slope, the differences between salinity climatologies (Figure $5 \mathrm{f}$ ) are near zero and freshening occurs at a slower rate $\left(\approx-0.4 \times 10^{-2} \mathrm{psu} \mathrm{yr}^{-1}--0.3 \times 10^{-2} \mathrm{psu} \mathrm{yr}^{-1}\right)$ than offshore waters, with a maximum negative trend of $\approx-0.6 \times \mathrm{psu} \mathrm{yr}^{-1}$ (Figure $7 \mathrm{~b}$ ).

Both warming and freshening across the domain contribute to the overall decreasing in water density (Figure $7 \mathrm{c}$ ). However, following the salinity trends, there is a slightly slower decrease rate in density over the slope $(\approx$ $-2.5 \times 10^{-3} \mathrm{~kg} \cdot \mathrm{m}^{3} \cdot \mathrm{yr}^{-1}--4.7 \times 10^{-3} \mathrm{~kg} \cdot \mathrm{m}^{3} \cdot \mathrm{yr}^{-1}$; Figure $7 \mathrm{c}$ ).

In the outflow there is an even higher decrease in density, concentrated over the continental shelf, where it differs up to $-0.44 \mathrm{~kg} / \mathrm{m}^{3}$ from the first decade (Figure $6 \mathrm{f}$ ) and reaches the maximum decreasing trend of $-6.6 \times 10^{-3}$ $\mathrm{kg} \cdot \mathrm{m}^{3} \cdot \mathrm{yr}^{-1}$. Different from the inflow, where there is a widespread warming throughout the water column, the increase in surface temperature at the outflow, in both onshore and offshore waters, converge over the slope, at the ASF location, and follows towards the bottom where it reaches the maximum warming trend of $\approx 0.015^{\circ} \cdot \mathrm{yr}^{-1}$ (Figure $7 \mathrm{~d}$ ). Over the continental shelf, where the waters has become less dense, there is smaller but significant trend of $\leq 0.005^{\circ} \mathrm{C}$ yr -1 down to $\approx 200 \mathrm{~m}$ depth, but the layer below that shows significant cooling of $-0.003^{\circ} \mathrm{C} \cdot \mathrm{yr}^{-1}$.

This warming over the slope is also related with the warmer WDW expansion towards the coast. In the first decade the maximum temperature reached by this water mass is $0.68^{\circ} \mathrm{C}$ and its upper limit is found at $210 \mathrm{~m}$ depth offshore and does not intersect with the continental slope (Figure 5 d). However, in the last decade, besides reaching a temperature of $1.27^{\circ} \mathrm{C}$, it shoals to a depth around $180 \mathrm{~m}$ on the offshore waters and intersects with the slope at $750 \mathrm{~m}$ depth (Figure $5 \mathrm{e}$ ).

On the other hand, the salinity in the outflow, as well as the inflow, shows a freshening over the entire domain (Figure $7 \mathrm{e}$ ). However, the most expressive freshening rate is found over the continental $\left(\approx-0.8 \times 10^{-2} \mathrm{psu} \cdot \mathrm{yr}^{-1}\right)$. Therefore, in contrast with inflow, the decrease in density in the outflow is linked with physical processes that induce a larger change in salinity than in temperature, such as changes in sea ice melting (Schmidtko et al., 2014), 
which is consistent with the sea ice variability discussed earlier.

The outflow also shows significant change in the geometry of the isopycnals. At the beginning of the century there is a marked V-shaped frontal structure (Figure $6 \mathrm{~d}$ ), characteristic of a Dense Shelf regime. In the last decade, although the isopycnals that slope from offshore waters towards the coast remain steep, the density surfaces that tilt from the coast are almost horizontal. This is a result of density decrease over the continental shelf (Figure $6 \mathrm{e})$. Along the shelf break $\left(\approx 54.5^{\circ} \mathrm{W}\right)$, down to $600 \mathrm{~m}$ depth, there is less of a V-shaped structure. This change suggests an evolution towards the configuration found in regions of Fresh Shelf regime like in the inflow region, where there is a stronger front.

In the inflow region, weakened easterlies reduced the downward Ekman pumping towards the coast, which combined with a less dense water column, led to the decrease in the lateral density gradient of the ASC and, therefore, the slowdown of geostrophic currents.

Changes in the outflow region, in turn, are mainly driven by the decrease in salinity over the continental shelf. Results obtained for this region are consistent with the observed trends found by Schmidtko et al. (2014) in the western WS Antarctic Shelf Bottom Water, where there has been slight cooling $\left(-0.05 \pm 0.04^{\circ} \mathrm{C} \cdot \mathrm{dec}^{-1}\right)$ and freshening $\left(-0.01 \pm 0.007 \mathrm{~g} \cdot \mathrm{kg}^{-1} \cdot \mathrm{dec}^{-1}\right)$ since 1990s. The freshening over the continental shelf reduced its vertical stratification and the tilting of the isopycnals towards offshore waters. On the other hand, this change over the shelf has strengthened the lateral gradient, and led to the transition towards a Fresh Shelf regime. This result also showed to be consistent with results obtained by Moorman et al. (2020) through a high-resolution experiment for a scenario of enhanced meltwater forcing. As well as in their results, in the outflow we have found a weakening trend in the cross-slope exchange due to a stronger ASC frontal structure, which explains the cooling response and the increase of the geostrophic currents.

\section{Conclusions}

In this study we investigated the ASC system response to climate change through a high-resolution experiment for the RCP8.5 scenario in the Weddell Sea. The simulation results were analyzed for two distinct regions (inflow and outflow).

In the inflow region, the ASC is classified as Fresh Shelf regime, characterized by a strong frontal structure. This region undergoes a weakening in the 
lateral density gradient driven mainly by the decrease in the Easterly winds magnitude. As a result, we have a weakened ASC system, which allows more flow of the warmer and fresher WDW flow towards the coast.

Our results suggest a southward strengthening of the westerlies from the beginning to the end of the century. Although the wind magnitude along the outflow region has increased, at the inflow region it undergoes a weakening $\left(\approx-1.3 \mathrm{~m} \cdot \mathrm{s}^{-1}\right.$ from the first to last decade). This change in the wind pattern is consistent with the SAM positive trend, which is likely to become more positive in a climate change scenario (Arblaster and Meehl, 2006; Thompson et al., 2011).

In the outflow region, although the increase in the winds magnitude contribute to a strengthened along-slope northward flux, greatest changes are due to the freshening over the continental shelf $\left(\approx-0.8 \times 10^{-2} \mathrm{psu}^{\circ} \mathrm{yr}^{-1}\right)$. This region experiences a larger variability in sea ice and, therefore, there is an enhanced freshwater flux which induces a decrease in salinity. This change over the continental shelf has increased the lateral density gradient, and in contrast with the inflow region, it has strengthened the ASC system. As a result, the ASC, which is currently classified as a Dense Shelf regime at the outflow, showed a transition towards a Fresh Shelf regime. This transition reduces the cross-slope exchange and, therefore, the onshore flux of WDW, which explains the cooling response of the shelf bottom water.

The results obtained in this study show opposite responses in the ASC system in the inflow and outflow regions. These changes can significantly modify the ASC dynamics and lead to changes in processes with global impact. The weakening in the ASC system, such as that found in the inflow region, makes it more susceptible to warming, which can accelerate the ice volume loss and directly impact the sea level rise. The ice loss increases the freshwater flux into the Antarctic coastal seas, which could alter the AABW formation by both decreasing in DSW formation and strengthening the ASC frontal system, which reduces the interaction between coastal and offshore waters, similar to what is found in the outflow region.

Despite the limitations of our model, which does not fully resolve the mesoscale eddy field and does not include tides, the results obtained in this study provide some insights on the different ASC responses to changes in large scale processes. Recent studies (Moorman et al., 2020; Morrison et al., 2020) have shown progress of high-resolution ocean and sea ice shelf modeling tools, that allow robust representation of the Antarctic coastal processes. This improvement can provide better understanding of regional changes throughout 
Antarctic coastal regions and their remote feedbacks, and also the possibility to develop improved downscaling experiments in the future, integrating processes that occur at different scales around Antarctica. The development and progress of numerical tools can significantly improve our understanding about the ASC dynamics and variability, and therefore, the impact of climate change over this region.

\section{Acknowledgments}

This study was supported in part by the Grants FAPESP:2018/14789-9; CNPq: 301726/2013-2, 405869/20134; CNPq.MCT.INCT.CRIOSFERA 573720/20088 and Coordenação de Aperfeiçoamento de Pessoal de Nível Superior Brasil (CAPES) Finance Code 001 CNPq/MCT_INCT-CRIOSFERA 465680/2014. We acknowledge the German Climate Computing Center (DKRZ) from Max Planck Institute for Meteorology in conducting the model experiments and making the MPI-ESM-MR data available. We thank Dr. J. E. Pereira for conducting the downscaling experiment. 


\section{Appendix A. Validation}

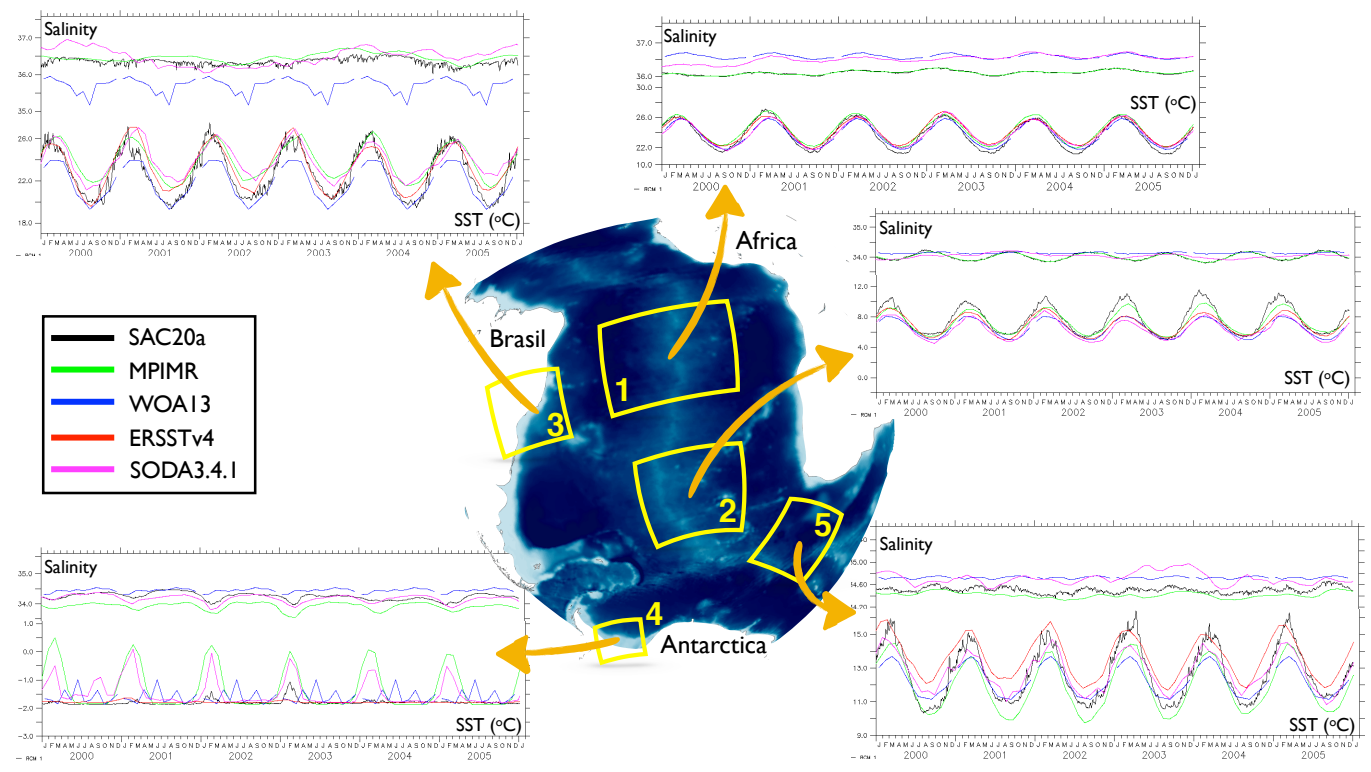

Figure A.1: Sea Surface Temperature (SST) and Sea Surface Salinity (SSS) regional means. The annual cycles and trends of both SST and SSS are accurately reproduced by our high-resolution experiment. Deviations between observed and modeled values are directly correlated with deviations in the global atmospheric forcings.

\section{References}

Arblaster, J.M., Meehl, G.A., 2006. Contributions of external forcings to southern annular mode trends. Journal of Climate 19, 2896-2905.

Budgell, W.P., 2005. Numerical simulation of ice-ocean variability in the barents sea region. Ocean Dynamics 55, 370-387. doi:10.1007/ s10236-005-0008-3.

Carmack, E.C., 1974. A quantitative characterization of water masses in the weddell sea during summer. Deep Sea Research and Oceanographic Abstracts 21, 431-443.

Comiso, J.C., Gersten, R.A., Stock, L.V., Turner, J., Perez, G.J., Cho, K., 2017. Positive trend in the antarctic sea ice cover and associated changes 
in surface temperature. Journal of Climate 30, 2251-2267. doi:10.1175/ JCLI-D-16-0408.1.

Dinniman, M.S., Asay-Davis, X.S., Galton-Fenzi, B.K., Holland, P.R., Jenkins, A., Timmermann, R., 2016. Modeling ice shelf/ocean interaction in antarctica: A review. Oceanography 29, 144-153. doi:10.5670/oceanog. 2016.106.

Dinniman, M.S., Klinck, J.M., Hofmann, E.E., 2012. Sensitivity of circumpolar deep water transport and ice shelf basal melt along the west antarctic peninsula to changes in the winds. Journal of Climate 25, 4799-4816. doi:10.1175/JCLI-D-11-00307.1.

Fahrbach, E., Hoppema, M., Rohardt, G., Boebel, O., Klatt, O., Wisotzki, A., 2011. Warming of deep and abyssal water masses along the greenwich meridian on decadal time scales: The weddell gyre as a heat buffer. Deep Sea Research Part II: Topical Studies in Oceanography 58, 2509-2523. doi:10.1016/j.dsr2.2011.06.007.

Fahrbach, E., Hoppema, M., Rohardt, G., Schröder, M., Wisotzki, A., 2004. Decadal-scale variations of water mass properties in the deep weddell sea. Ocean Dynamics 54, 77-91. doi:10.1007/s10236-003-0082-3.

Fahrbach, E., Rohardt, G., Krause, G., 1992. The antarctic coastal current in the southeastern wedell sea. Polar Biology 12, 171-182.

Fahrbach, E., Rohardt, G., Scheele, N., Schröder, M., Strass, V., Wisotzki, A., 1995. Formation and discharge of deep and bottom water in the northwestern weddell sea. Journal of Marine Research 53, 515-538. doi:10.1357/002224099321514060.

Flexas, M.d.M., Schodlok, M.P., Padman, L., Menemenlis, D., Orsi, A.H., 2015. Role of tides on the formation of the a ntarctic slope front at the weddell-scotia confluence. Journal of Geophysical Research: Oceans 120, 3658-3680. doi:10.1002/2014JC010372.

Frankcombe, L.M., Spence, P., Hogg, A.M., England, M.H., Griffies, S.M., 2013. Sea level changes forced by southern ocean winds. Geophysical Research Letters 40, 5710-5715. doi:10.1002/2013GL058104. 
Galton-Fenzi, B.K., Hunter, J.R., Coleman, R., Marsland, S.J., Warner, R.C., 2012. Modeling the basal melting and marine ice accretion of the amery ice shelf. Journal of Geophysical research 117. doi:10.1029/ 2012 JC008214.

Garabato, A.C.N., McDonagh, E.L., Stevens, D.P., Heywood, K.J., Sanders, R.J., 2002. On the export of antarctic bottom water from the weddell sea. Deep Sea Research Part II: Topical Studies in Oceanography 49, 47154742 .

Hattermann, T., Smedsrud, L.H., Nøst, O.A., Lilly, J.M., Galton-Fenzi, B.K., 2014. Eddy-resolving simulations of the fimbul ice shelf cavity circulation: Basal melting and exchange with open ocean. Ocean Modelling 82, 28-44. doi:10.1016/j.ocemod.2014.07.004.

Hellmer, H.H., Huhn, O., Gomis, D., Timmermann, R., 2011. On the freshening of the northwestern weddell sea continental shelf. Ocean Science 7, 305-316. doi:10.5194/os-7-305-2011.

Heywood, K.J., Garabato, A.C.N., Stevens, D.P., Muench, R.D., 2004. On the fate of the antarctic slope front and the origin of the weddell front. Journal of Geophysical Research 109, C06021. doi:10.1029/2003JC002053.

Hock, R., De Woul, M., Radic, V., Dyurgerov, M., 2009. Mountain glaciers and ice caps around antarctica make a large sea-level rise contribution. Geophysical Research Letters 36, 1-5. doi:10.1029/2008GL037020.

Hogg, A.M., Meredith, M.P., Chambers, D.P., Abrahamsen, E.P., Hughes, C.W., Morrison, A.K., 2015. Recent trends in the s outhern ocean eddy field. Journal of Geophysical Research: Oceans 120, 257-267. doi:10. 1002/2014JC010470.

Jacobs, S.S., 1991. On the nature and significance of the antarctic slope front. Marine Chemistry 35, 9-24.

Jungclaus, J., Fischer, N., Haak, H., Lohmann, K., Marotzke, J., Matei, D., Mikolajewicz, U., Notz, D., Von Storch, J., 2013. Characteristics of the ocean simulations in the max planck institute ocean model (mpiom) the ocean component of the mpi-earth system model. Journal of Advances in Modeling Earth Systems 5, 422-446. doi:10.1002/jame.20023. 
Kerr, R., Mata, M.M., Garcia, C.A.E., 2009. On the temporal variability of the weddell sea deep water masses. Antarctic Science 21, 383-400. doi:10.1017/S0954102009001990.

Kwok, R., Comiso, J.C., 2002. Spatial patterns of variability in antarctic surface temperature: Connections to the southern hemisphere annular mode and the southern oscillation. Geophysical Research Letters 29, 50-1-50-4. doi:10.1029/2002GL015415.

Marshall, G.J., 2003. Trend in the southern annular mode from observations and reanalyses. Journal of Climate 16, 4134-4143.

Marshall, J., Speer, K., 2012. Closure of the meridional overturning circulation through Southern Ocean upwelling. Nature Geoscience 5, 171-180. doi:10.1038/ngeo1391.

Meehl, G.A., Arblaster, J.M., Chung, C.T.Y., Holland, M.M., DuVivier, A., Thompson, L., Yang, D., Bitz, C.M., 2019. Sustained ocean changes contributed to sudden antarctic sea ice retreat in late 2016. Nature Communications 10, 1-9. doi:10.1038/s41467-018-07865-9.

Meijers, A., Meredith, M., Abrahamsen, E., Morales Maqueda, M., Jones, D., Naveira Garabato, A., 2016. Wind-driven export of $\mathrm{w}$ eddell s ea slope water. Journal of Geophysical Research: Oceans 121, 7530-7546. doi:10.1002/2016 JC011757.

Moorman, R., Morrison, A.K., Hogg, A.M., 2020. Thermal responses to antarctic ice shelf melt in an eddy-rich global ocean-sea ice model. Journal of Climate 33, 6599-6620. doi:10.1175/JCLI-D-19-0846.1.

Morrison, A.K., Hogg, A.M., England, M.H., Spence, P., 2020. Warm circumpolar deep water transport toward antarctica driven by local dense water export in canyons. Science Advances 6.

Núñez-Riboni, I., Fahrbach, E., 2009. Seasonal variability of the antarctic coastal current and its driving mechanisms in the weddell sea. Deep-Sea Research Part I: Oceanographic Research Papers 56, 1927-1941. doi:10. 1016/j.dsr.2009.06.005.

Nøst, O.A., Biuw, M., Tverberg, V., Lydersen, C., Hattermann, T., Zhou, Q., Smedsrud, L.H., Kovacs, K.M., 2011. Eddy overturning of the antarctic 
slope front controls glacial melting in the eastern weddell sea. Journal of Geophysical Research: Oceans 116. doi:10.1029/2011JC006965.

Orsi, A.H., Johnson, G.C., Bullister, J.L., 1999. Circulation,mixing and production of antarctic bottom water. Progress in Oceanography 43, 55109.

Orsi, A.H., Nowlin, W.D., Whitworth, T., 1993. On the circulation and stratification of the weddell gyre. Deep Sea Research 40, 169-203.

Paolo, F.S., Fricker, H.A., Padman, L., 2015. Volume loss from antarctic ice shelves is accelerating. Science 348, $327-331$.

Purkey, S.G., Johnson, G.C., 2010. Warming of global abyssal and deep southern ocean waters between the 1990s and 2000s: Contributions to global heat and sea level rise budgets*. Journal of Climate 23, 6336-6351. doi:10.1175/2010JCLI3682.1.

Purkey, S.G., Johnson, G.C., 2012. Global contraction of antarctic bottom water between the 1980s and 2000s*. Journal of Climate 25, 5830-5844. doi:10.1175/JCLI-D-11-00612.1.

Rignot, E., Mouginot, J., Scheuchl, B., van den Broeke, M., van Wessem, M.J., Morlighem, M., 2019. Four decades of antarctic ice sheet mass balance from 1979-2017. Proceedings of the National Academy of Sciences 116, 1095-1103. doi:10.1073/pnas.1812883116.

Rintoul, S.R., Hughes, C., Olbers, D., 2001. The Antarctic Circumpolar Current System. Academic Press. pp. $271-302$.

Robertson, R., Visbeck, M., Gordon, A.L., Fahrbach, E., 2002. Long term temperature trends in the deep waters of the weddell sea. Deep-Sea Res. II 49, 4791-4806.

Schmidtko, S., Heywood, K.J., Thompson, A.F., Aoki, S., 2014. Multidecadal warming of Antarctic waters. Science 346, 1227-1231. doi:10. 1126/science. 1256117.

Schröder, M., Fahrbach, E., 1999. On the structure and the transport of the eastern weddell gyre. Deep-Sea Research Part II: Topical Studies in Oceanography 46, 501-527. doi:10.1016/S0967-0645(98)00112-X. 
Shchepetkin, A.F., 2003. A method for computing horizontal pressuregradient force in an oceanic model with a nonaligned vertical coordinate. Journal of Geophysical Research 108, 3090. doi:10.1029/2001JC001047.

Shchepetkin, A.F., McWilliams, J.C., 2005. The regional oceanic modeling system (roms): A split-explicit, free-surface, topography-followingcoordinate oceanic model. Ocean Modelling 9, 347-404. doi:10.1016/ j.ocemod. 2004.08 .002 .

Smith, W.H., Sandwell, D.T., 1997. Global sea floor topography from satellite altimetry and ship depth soundings. Science 277, 1956-1962.

Spence, P., Griffies, S.M., England, M.H., Hogg, A.M., Saenko, O.A., Jourdain, N.C., 2014. Rapid subsurface warming and circulation changes of antarctic coastal waters by poleward shifting winds. Geophysical Research Letters 41, 4601-4610. doi:10.1002/2014GL060613.

Spence, P., Holmes, R.M., Hogg, A.M., Griffies, S.M., Stewart, K.D., England, M.H., 2017. Localized rapid warming of west antarctic subsurface waters by remote winds. Nature Climate Change 7, 595-603. doi:10.1038/NCLIMATE3335.

Stewart, A.L., Thompson, A.F., 2015. Eddy-mediated transport of warm circumpolar deep water across the antarctic shelf break. Geophysical Research Letters 42, 432-440. doi:10.1002/2014GL062281.

Sverdrup, H.U., 1953. The currents off the coast of queen maud land. doi:10 . $1080 / 00291955308542731$.

Thompson, A.F., Heywood, K.J., Schmidtko, S., Stewart, A.L., 2014. Eddy transport as a key component of the antarctic overturning circulation. Nature Geoscience 7, 171-180. doi:10.1038/NGE02289.

Thompson, A.F., Stewart, A.L., Spence, P., Heywood, K.J., 2018. The antarctic slope current in a changing climate. Reviews of Geophysics 56, 741-770. doi:10.1029/2018RG000624.

Thompson, D.W.J., Solomon, S., Kushner, P.J., England, M.H., Grise, K.M., Karoly, D.J., 2011. Signatures of the antarctic ozone hole in southern hemisphere surface climate change. Nature Geoscience 4, 741-749. doi:10. 1038/ngeo1296. 
Thompson, D.W.J., Wallace, J.M., 2000. Annular mode in the extratropical circulation. part i : Month-to-month variability. Journal of Climate 13, 1000-1016. doi:http://dx.doi.org/10.1175/1520-0442(2000) 013<1000: AMITEC> 2.0 . CO 2 .

Tonelli, M., Marcello, F., Ferrero, B., Wainer, I., 2019. Warm deep water variability during the last millennium in the cesm-lme: Pre-industrial scenario versus late 20th century changes. Geosciences 9, 346. doi:10.3390/ geosciences 9080346 .

Turner, J., Guarino, M.V., Arnatt, J., Jena, B., Marshall, G.J., Phillips, T., Bajish, C.C., Clem, K., Wang, Z., Andersson, T., Murphy, E.J., Cavanagh, R., 2020. Recent decrease of summer sea ice in the weddell sea, antarctica. Geophysical Research Letters 47, e2020GL087127. doi:10.1029/2020GL087127.

Turner, J., Hosking, J.S., Bracegirdle, T.J., Marshall, G.J., Phillips, T., 2015. Recent changes in antarctic sea ice. Philosophical Transactions of the Royal Society A: Mathematical, Physical and Engineering Sciences 373, 20140163. doi:10.1098/rsta.2014.0163.

Turner, J., Hosking, J.S., Phillips, T., Marshall, G.J., 2013. Temporal and spatial evolution of the antarctic sea ice prior to the september 2012 record maximum extent. Geophysical Research Letters 40, 5894-5898. doi:10. 1002/2013GL058371.

Turner, J., Phillips, T., Marshall, G.J., Hosking, J.S., Pope, J.O., Bracegirdle, T.J., Deb, P., 2017. Unprecedented springtime retreat of antarctic sea ice in 2016. Geophysical Research Letters 44, 6868-6875. doi:10.1002/ 2017 GL073656.

Whitworth, T., Orsi, A.H., Kim, S.J., Nowlin, W.D., Locarnini, R.A., 1998. Water masses and mixing near the antarctic slope front, in: Jacobs, S.S., Weiss, R.F. (Eds.), Ocean, Ice, and Atmosphere: Interactions at the Antarctic Continental Margin. American Geophysical Union. volume 75, pp. 1-27. URL: http://dx.doi.org/10.1029/AR075p0001, doi:10.1029/AR075p0001.

Wilkin, J., Hedstrom, K., 1998. User's manual for an orthogonal curvilinear grid-generation package. Technical Report. Institute of Marine and Coastal 
732 Sciences, Rutgers University. URL: http://www.marine.rutgers.edu/ 733 po/tools/gridpak/grid_manual.ps.gz. 